\title{
Aerodynamic Benefits of Near-Surface-Actuated Circulation Control Blowing Slots for Rotorcraft Use
}

Gerald M. Angle II

West Virginia University

Follow this and additional works at: https://researchrepository.wvu.edu/etd

\section{Recommended Citation}

Angle, Gerald M. II, "Aerodynamic Benefits of Near-Surface-Actuated Circulation Control Blowing Slots for Rotorcraft Use" (2008). Graduate Theses, Dissertations, and Problem Reports. 4356.

https://researchrepository.wvu.edu/etd/4356

This Dissertation is protected by copyright and/or related rights. It has been brought to you by the The Research Repository @ WVU with permission from the rights-holder(s). You are free to use this Dissertation in any way that is permitted by the copyright and related rights legislation that applies to your use. For other uses you must obtain permission from the rights-holder(s) directly, unless additional rights are indicated by a Creative Commons license in the record and/ or on the work itself. This Dissertation has been accepted for inclusion in WVU Graduate Theses, Dissertations, and Problem Reports collection by an authorized administrator of The Research Repository @ WVU.

For more information, please contact researchrepository@mail.wvu.edu. 


\title{
Aerodynamic Benefits of Near-Surface-Actuated Circulation Control Blowing Slots for Rotorcraft Use
}

\author{
Gerald M. Angle II, MSAE
}

\author{
A Dissertation Submitted to the \\ College of Engineering and Mineral Resources \\ At West Virginia University \\ In Partial Fulfillment of the Requirements \\ For the Degree of
}

\author{
Doctor of Philosophy \\ in \\ Aerospace Engineering
}

Department: Mechanical and Aerospace Engineering

Major: Aerospace Engineering

\author{
West Virginia University \\ Morgantown, WV \\ 2008
}

Keywords: Circulation Control, Helicopter Rotor Lift

Copyright 2008 Gerald M. Angle II 


\section{Abstract}

Aerodynamic Benefits of Near-Surface-Actuated Circulation Control Blowing Slots for Rotorcraft Use

Gerald M. Angle II

One of the most maintenance intensive components of all present-day helicopters is the swash-plate, and its associated control linkages. This mechanism is also the major contributing factor to fatal accidents associated with helicopter flight. However, the swash-plate is an essential component in providing controlled flight, by transmitting the pilot's control-inputs to the main rotor. Another side-effect of using the swash-plate is that it increases the diameter of the main rotor shaft assembly, slightly increasing the total drag of the aircraft. Thus, there are several benefits that could be achieved by removing the swash-plate of the helicopter.

To enable the removal of the swash-plate from a rotorcraft, a method is needed to augment the aerodynamic performance of the main rotor blades as a function of azimuth angle, while maintaining the traditional cyclic and collective pilot controls. Circulation control can provide a means of changing the aerodynamic performance without physically changing the angle of attack of the blade, as is currently done by the swash-plate. Thus, the current project was conceived to investigate the use of surface-mounted valves to control leading and trailing edge circulation control blowing as a means of decreasing the flow establishment time for circulation control wing lift augmentation.

Both experimental and computational methods were implemented in this study. From the experimental results, a lift augmentation ratio $\left(\mathrm{C}_{1} / \mathrm{C}_{\mu}\right)$ of 47 could be achieved in $56 \mathrm{msec}$, with the model at an angle of attack of 0.68 degrees. The computational study yielded a force establishment time of 55 to $60 \mathrm{msec}$ to achieve a lift coefficient greater than 5 at 0 degree angle of attack. From this data it is concluded that the use of a circulation control airfoil, with blowing jet controlled at or near the airfoil surface, can effectively be used on a helicopter main rotor if the rotor speed is less than $275 \mathrm{rpm}$ with four defined force control points per revolution. The rotational speed is within the operational range of current technology rotor speeds. 


\section{Table of Contents}

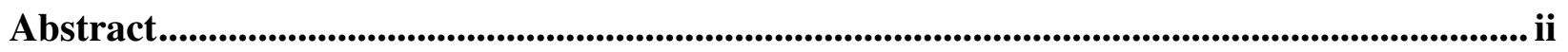

Table of Contents ...............................................................................................................................iii

List of Figures.......................................................................................................................................... $v$

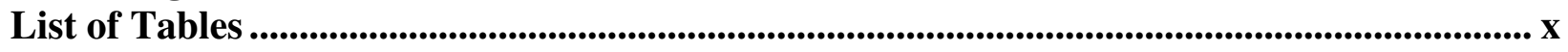

Nomenclature ........................................................................................................................................ xii

1. Introduction............................................................................................................................ 1

2. Research Objectives............................................................................................................................ 4

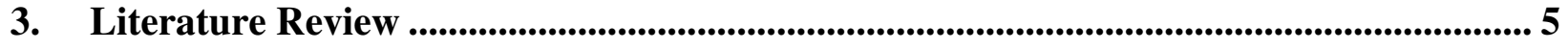

3.1. Basic Fluid Flow Relationships ……………................................................... 5

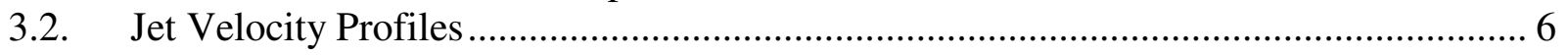

3.3. Boundary Layer Profiles ................................................................................ 9

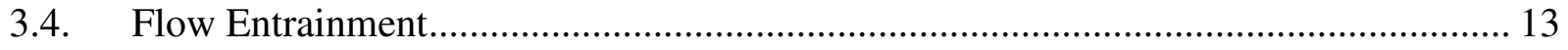

3.5. Separation Location Prediction ............................................................................. 14

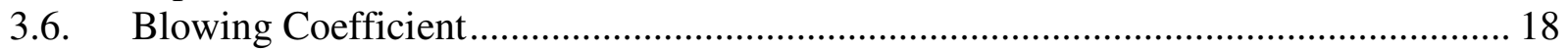

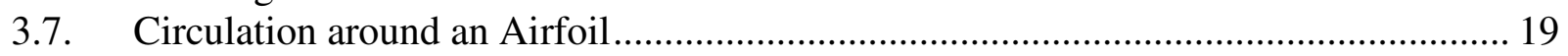

3.8. Circulation Controlled Airfoils ……………….................................................. 20

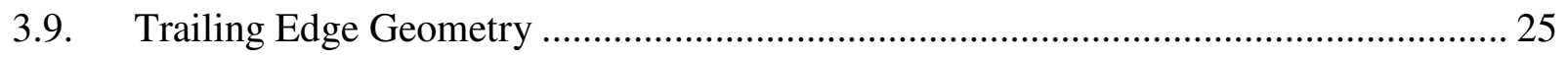

3.10. Impact of Sweep Angle on Circulation Control ....................................................... 26

3.11. Sources of Error in Circulation Control Investigations ............................................. 27

3.12. Computational Modeling of Circulation Control...................................................... 29

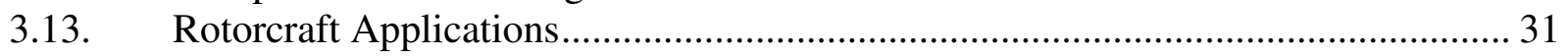

3.13.1. Reverse Blowing Circulation Control Rotor............................................................ 33

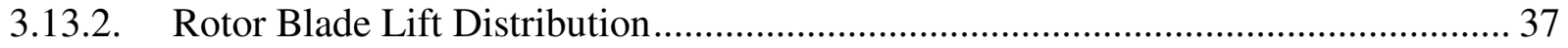

3.13.3. Flow Development Response Time ....................................................................... 37

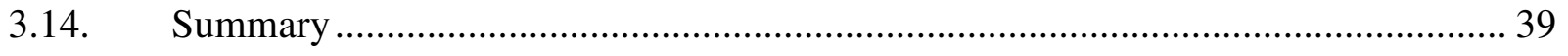

4. Preliminary Calculations............................................................................................................................. 40

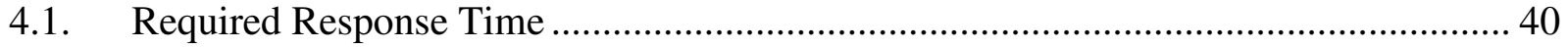

4.2. Blade Filling Time Estimation............................................................................... 41

4.3. Comparable Airfoil NACA 0010-34 ................................................................... 44

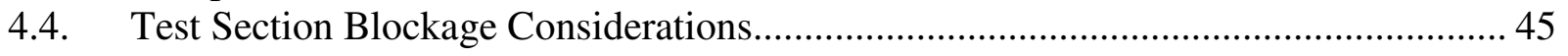

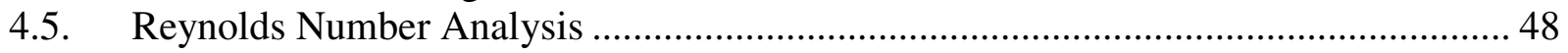

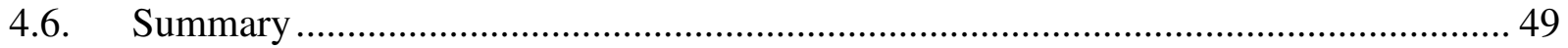

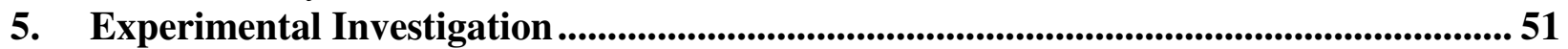

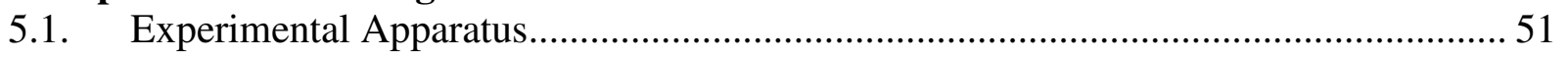

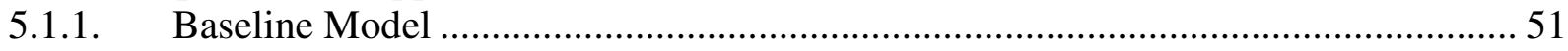

5.1.2. Active Circulation Control Model …………….................................................. 55

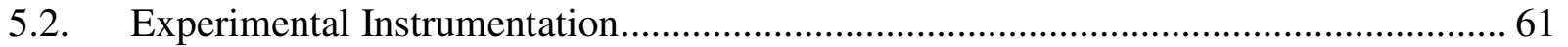

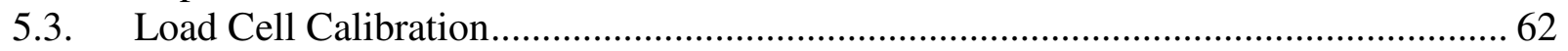

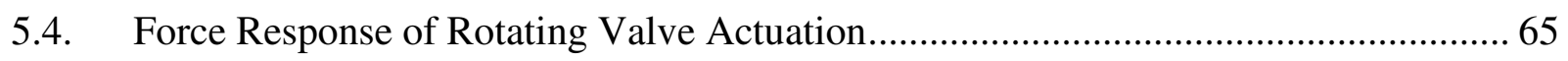

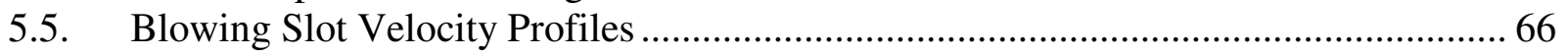

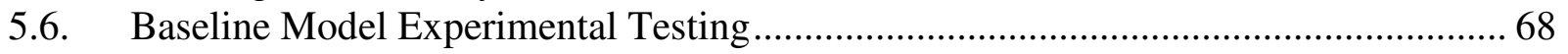

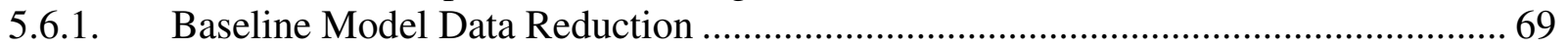

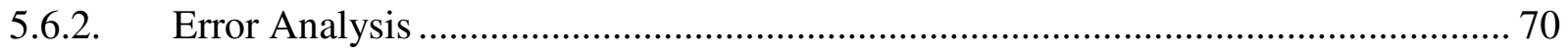




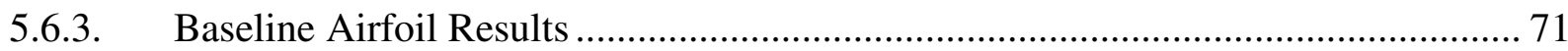

5.7. Active Circulation Control Model Experimental Testing ............................................. 73

5.7.1. Active Model Testing Procedure ....................................................................... 74

5.7.2. Active Model Experimental Results …………….................................................. 75

5.7.2.1. Aerodynamic Force Coefficient Comparisons..................................................... 75

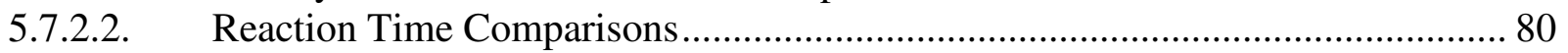

5.7.3. Error Analysis ............................................................................................... 83

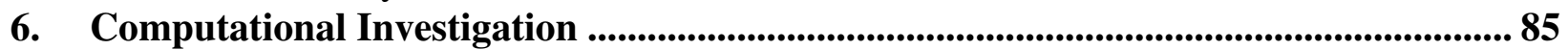

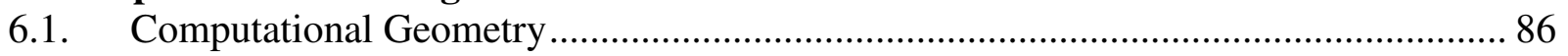

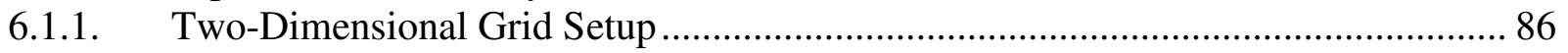

6.1.2. Three Dimensional Grid Setup ………………................................................. 90

6.2. Computational Solver Specifics........................................................................ 92

6.2.1. Two-Dimensional Solver Settings ........................................................................ 92

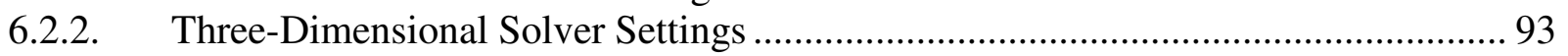

6.3. Computational Grid Independence …………….................................................. 93

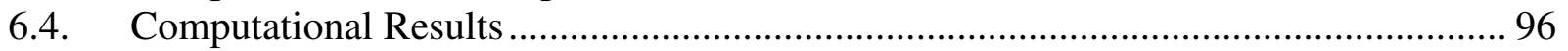

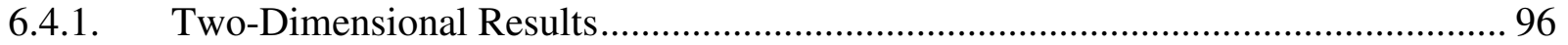

6.4.2. Unsteady Two-Dimensional Time Response Results.............................................. 106

6.4.3. Three-Dimensional Results.............................................................................. 109

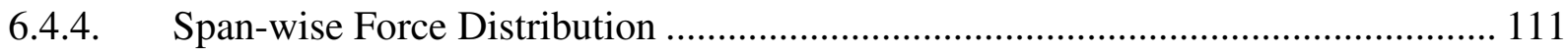

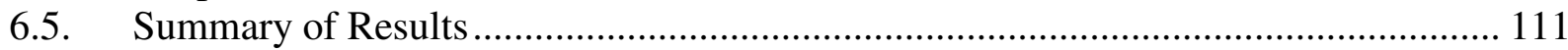

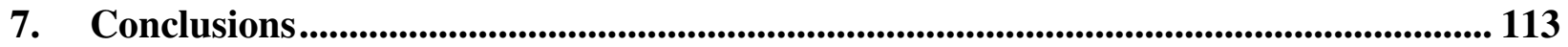

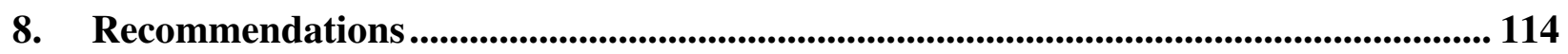

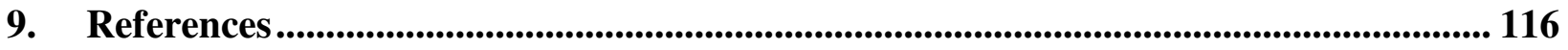

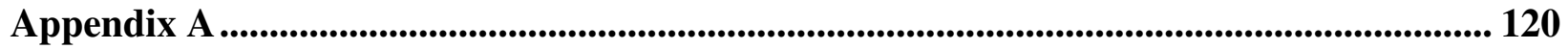

Appendix B .................................................................................................................................. 124

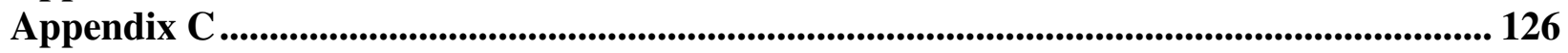

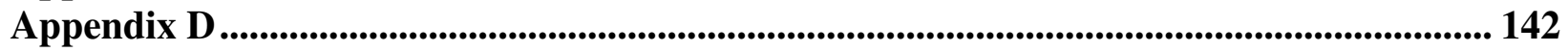

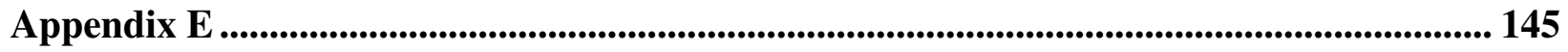

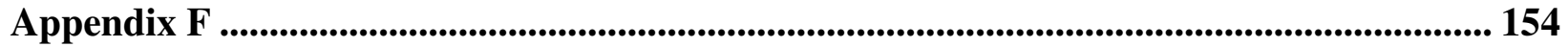

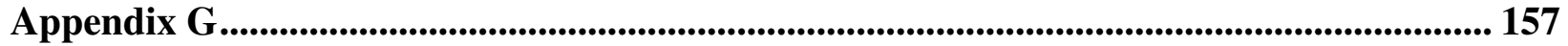

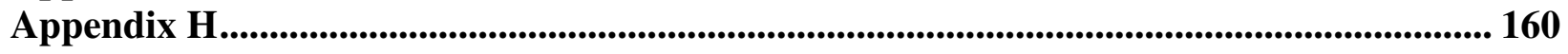

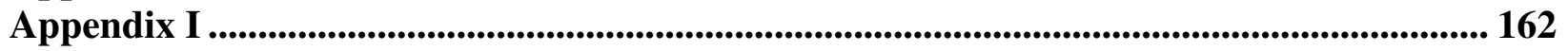




\section{List of Figures}

Figure 3.1: Two-dimensional free turbulent jet, Newman [1961] ............................................... 7

Figure 3.2: Two-dimensional turbulent wall jet, Newman [1961] .......................................... 7

Figure 3.3: Re-attachment of a two-dimensional jet to an inclined flat plate, Newman [1961] ..... 7

Figure 3.4: Flow of a two-dimensional jet round a circular cylinder, Newman [1961] ................ 11

Figure 3.5: Boundary layer development of a wall jet, Gibbs [1975] ...................................... 11

Figure 3.6: Boundary layer-jet interaction over a Coanda surface, Myer [1972] ......................... 12

Figure 3.7: The position of separation for small b/a, Newman [1961] .................................... 17

Figure 3.8: Comparison of theory presented in Kind [1968] to experimental curves presented in

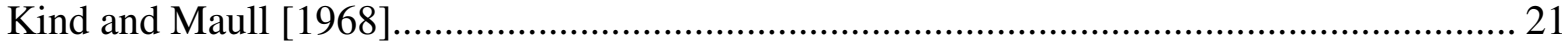

Figure 3.9: Lift (A) and drag (B) coefficients for a circulation controlled elliptical airfoil,

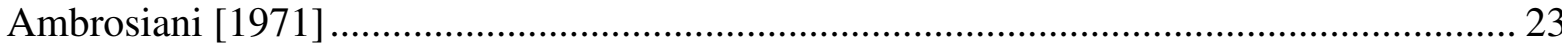

Figure 3.10: Root (A) and tip (B) airfoil profile shapes used in the reverse blowing - circulation

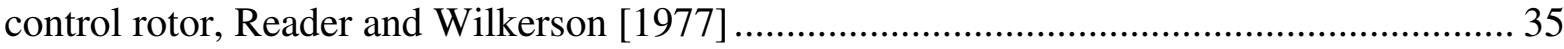

Figure 3.11: Reverse blowing circulation control rotor hub, Reader and Wilkerson [1977] ........ 36

Figure 3.12: Spanwise loading of a model rotor blade, Meyer and Falabella [1953]................... 37

Figure 4.1: Response time required based on typical rotor speeds ............................................. 41

Figure 4.2: Relationship between density and pressure for compressed air [The Engineering

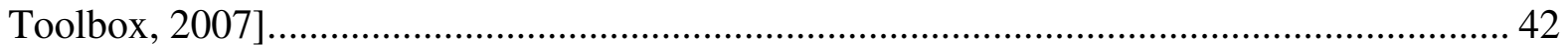

Figure 4.3: Plenum pressure of the system as dependent upon time ........................................... 43

Figure 4.4: Exit and inlet velocities of the system as dependent upon time.................................. 44

Figure 4.5: Profile comparison of the CCHB Airfoil to the NACA 0010-34............................. 45

Figure 4.6: Blockage Ratio in Percent for Basic Airfoil Shapes Considered for the CCHB as

Dependent Upon the Angle of Attack.................................................................................. 46

Figure 4.7: Blockage Ratio in Percent for Basic Airfoil Shapes Considered for the CCHB as

Dependent Upon the Chordlength ....................................................................................... 47

Figure 4.8: Local Reynolds Number for a Helicopter Rotor, with a one ft Chordlength, Spinning

at $200 \mathrm{rpm}$ as Dependent Upon the Blade Station .............................................................. 48

Figure 5.1: Top view of baseline elliptical airfoil model........................................................ 52

Figure 5.2: End view of baseline elliptic airfoil model with top cover and trailing edge removed

$\ldots$

Figure 5.3: Close-up view of the assembly method for the baseline elliptic airfoil model .......... 52

Figure 5.4: End view of assembled baseline elliptic airfoil model without endplates ................. 53

Figure 5.5: Close-up of surface pressure tap spacing along top surface of the baseline elliptic

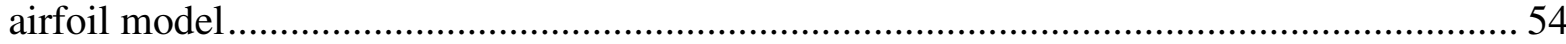

Figure 5.6: Baseline Model Installed in the WVU Closed Loop Wind Tunnel............................. 55

Figure 5.7: Cross-sectional schematic of 10:1 elliptic airfoil with internal plenums ................... 55

Figure 5.8: Two-dimensional model of circulation controlled helicopter blade with endplates .. 56

Figure 5.9: Block diagram of internal airflow of wind tunnel model .......................................... 56

Figure 5.10: Preliminary leading edge slot control configuration for wind tunnel model ........... 57

Figure 5.11: Second leading edge slot control configuration for wind tunnel model.................... 57

Figure 5.12: Final leading edge slot control configuration for wind tunnel model ....................... 58

Figure 5.13: Final trailing edge slot control configuration for wind tunnel model ......................5 58

Figure 5.14: Profile of radius for the three trailing edge slot control devices .............................. 59 
Figure 5.15: Leading Edge Solenoid Control Configuration for the Rotary Valve. 60

Figure 5.16: Close Up of CCHB Model Internal Components, Including the Air Inlets, and Porous Walls 60

Figure 5.17: Chordwise View of the CCHB Model Showing the Leading Edge Slot Structure and

Trailing Edge Rotating Device ..... 61

Figure 5.18: Upper Load Cell Configuration........................................................................ 62

Figure 5.19: Angle of Attack Setting Device Pinned at 0 Degree, Indicated Angle of Attack .... 62

Figure 5.20: Centerline Calibration Setup of the Baseline Model............................................ 63

Figure 5.21: Calibrations of the Four Load Cells with the Baseline Model:.............................. 64

Figure 5.22: Interference of Force Measurement Due to the Opposite Force ........................... 64

Figure 5.23: Measured Forces During Solenoid Opening of Both Blowing Slots in Bottom Drag

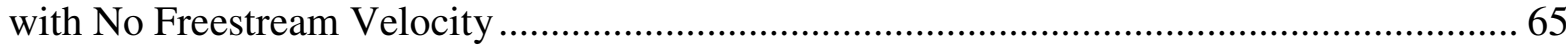

Figure 5.24: Measured Forces During Solenoid Opening of Leading Edge Blowing Slot in

Bottom Drag with No Freestream Velocity ................................................................ 66

Figure 5.25: Measured Forces During Solenoid Opening of Trailing Edge Blowing Slot in

Bottom Drag with No Freestream Velocity ................................................................... 66

Figure 5.26: Non-Dimensional Blowing Slot Exit Profile for the Leading Edge...................... 67

Figure 5.27: Non-Dimensional Blowing Slot Exit Profile for the Trailing Edge ........................ 68

Figure 5.28: Baseline Test Results Compared to the NACA 0010-34 Airfoil .......................... 72

Figure 5.29: Drag Coefficient Measured for the 10:1 Elliptic Airfoil Compared to the NACA

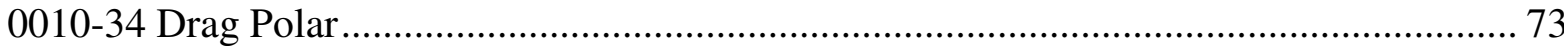

Figure 5.30: Jet Exit Velocity as Measured Based on the Regulated Pressure.......................... 74

Figure 5.31: Lift Curve Comparison of the Baseline and Active Models ................................ 76

Figure 5.32: Lift coefficient as dependent upon the blowing coefficient data at a Reynolds

number of $4 \times 10^{6}$, compared to the results from Kind and Maull [1968]............................. 77

Figure 5.33: Lift Coefficients for the CCHB Active Model at 0.68 Degrees Angle of Attack with

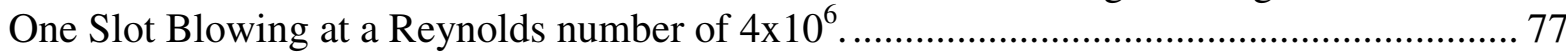

Figure 5.34: Lift and Drag Force Coefficients Measured for Various Blowing Coefficients for the

CCHB Active Model at 0.68 Degrees Angle of Attack.................................................... 78

Figure 5.35: Lift Coefficients for the CCHB Active Model at 0.68 Degrees Angle of Attack with

a Leading Edge Pressure of 10 psig ............................................................................ 79

Figure 5.36: Lift Coefficients for the CCHB Active Model at 0.68 Degrees Angle of Attack with

a Leading Edge Pressure of 20 psig .......................................................................... 79

Figure 5.37: Lift Coefficients for the CCHB Active Model at 0.68 Degrees Angle of Attack with

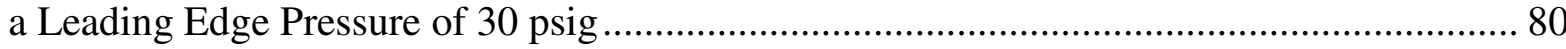

Figure 5.38: Solenoid Opening Time Requirement Measurement ........................................ 81

Figure 5.39: Experimental Time Response for Lift with the 30 psig Regulated Trailing Edge

Closing Test Condtion at 0.7 Degrees Angle of Attack.................................................. 82

Figure 5.40: Experimental Time Response for Drag with the 30 psig Regulated Trailing Edge

Closing Test Condition at 0.7 Degrees Angle of Attack ........................................... 82

Figure 5.41: Trailing Edge of the Active Model in the Closed Position ................................... 84

Figure 5.42: Trailing Edge of the Active Model in the Open Position..................................... 84

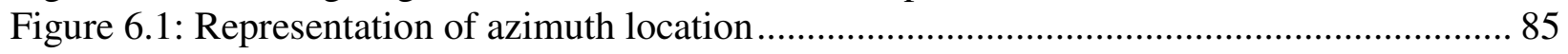

Figure 6.2: Computational space modeled for the two-dimensional study ............................. 87

Figure 6.3: Computational node points on and near the upper surface, trailing edge blowing slot 
Figure 6.4: Computational grid point locations on, and near, the upper surface, leading edge

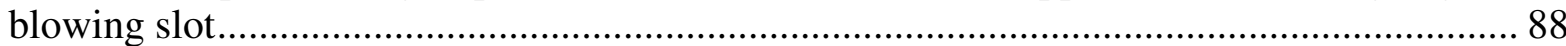

Figure 6.5: Locally enlarged view of the grid for the upper surface trailing edge blowing slot .. 88 Figure 6.6: Locally enlarged view of the 2-D grid for the upper surface leading edge blowing slot

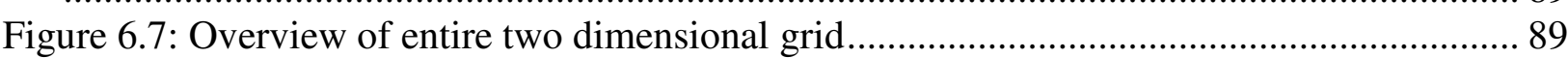

Figure 6.8: Extents of Three-Dimensional Volume for the 0 Degree Sweep CCHB Analysis .... 90 Figure 6.9: Extents of Three-Dimensional Volume for the 45 Degree Swept CCHB Analysis... 91 Figure 6.10: Variation in lift coefficient with respect to the number of computational node points

Figure 6.11: Variation in drag coefficient with respect to the number of computational node

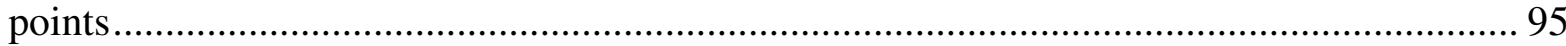

Figure 6.12: View of computational grid near the airfoil ............................................. 96

Figure 6.13: Trailing edge streamlines for trailing edge upper jet $\mathrm{C}_{\mu}=0.36$ and freestream

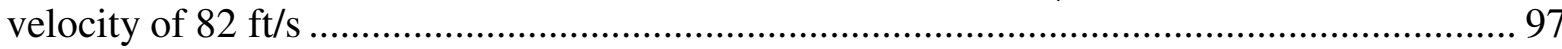

Figure 6.14: Comparison of Computational Lift Coefficient for the Trailing Edge Blowing to the Experimental and Kind and Maull [1968] Data.

Figure 6.15: Leading Edge Streamlines for Leading Edge Upper Jet $C_{\mu}$ of 0.16 and Freestream Velocity of $82 \mathrm{ft} / \mathrm{s}$ 100

Figure 6.16: Velocity Contours Near the Leading Edge of the CCHB Airfoil with Leading Edge Upper Surface Blowing Coefficient of 0.16 101

Figure 6.17:Streamlines for the Leading Edge Blowing at $\mathrm{C}_{\mu \_} \mathrm{LE}=0.027$ and Trailing Edge Blowing at $\mathrm{C}_{\mu \mathrm{TE}}=0.148$ 101

Figure 6.18: Computational Lift Coefficient for Various Leading Edge, Upper Surface Blowing Coefficient as Dependent Upon the Trailing Edge, Upper Surface Blowing Coefficient .... 102 Figure 6.19: Computational Lift Coefficient for Various Leading Edge, Lower Surface Blowing Coefficient as Dependent Upon the Trailing Edge, Upper Surface Blowing Coefficient .... 103 Figure 6.20: Computational Lift Coefficient for Total Blowing Coefficient with Leading and Trailing Edge, Upper Surface Blowing 104

Figure 6.21: Streamlines for Freestream Velocity of $1500 \mathrm{ft} / \mathrm{s}$, Leading Edge Upper Surface Blowing at $600 \mathrm{ft} / \mathrm{s}\left(\mathrm{C}_{\mu_{\_} L E}=0.0003\right)$ and Trailing Edge Upper Surface Blowing at $1400 \mathrm{ft} / \mathrm{s}$ $\left(\mathrm{C}_{\mu \_\mathrm{TE}}=0.001\right)$. 105

Figure 6.22: Steady State, Lift Coefficient as Dependent Upon the Freestream Velocity for Uniform Blowing Conditions 105

Figure 6.23: Time Response for Lift With Trailing Edge Upper Surface Blowing at $C_{\mu}=0.16106$ Figure 6.24: Time Response for Drag With Trailing Edge Upper Surface Blowing at $C_{\mu}=0.16$

Figure 6.25: Time Response for Lift With Leading Edge Upper Surface Blowing at $C_{\mu}=0.16107$ Figure 6.26: Time Response for Drag with Leading Edge Upper Surface Blowing at $C_{\mu}=0.16$

Figure 6.27: Time Response for Lift with Upper Surface Blowing on Both the Leading Edge at

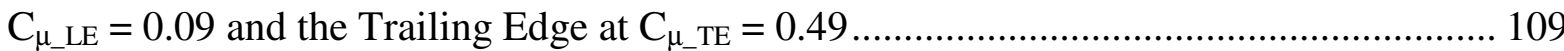

Figure 6.28: Time Response for Drag with Upper Surface Blowing on Both the Leading Edge at

$\mathrm{C}_{\mu_{-} \mathrm{LE}}=0.09$ and the Trailing Edge at $\mathrm{C}_{\mu_{-} \mathrm{TE}}=0.49$ 109

Figure 6.29: Velocity Contour along Centerline of Un-Swept Geometry with Trailing Edge Blowing Coefficient of 0.52 . 
Figure 6.30: Velocity Contour along Centerline of 45-Degree Swept Geometry with Trailing Edge Blowing Coefficient of 0.52.

Figure 6.31: Potential Lift Distribution for the Circulation Control Rotor Blade, in Hover and at a

Flight Speed of $100 \mathrm{mph}$ as Compared to the Data from Meyer and Falabella [1953]....... 111

Figure D. 1: Experimental Time Response Characteristics for Lift with the 30 psig Regulated

Trailing Edge Opening Test Condition at 0.7 Degrees Angle of Attack with an $82 \mathrm{ft} / \mathrm{s}$

Freestream Velocity

Figure D. 2: Lift Force for One Second Time Axis View for Experimental Time Response

Characteristics with the 30 psig Regulated Trailing Edge Opening Test Condition at 0.7

Degrees Angle of Attack with an $82 \mathrm{ft} / \mathrm{s}$ Freestream Velocity ....

Figure D. 3: Lift Force for One-Half Second Time Axis View for Experimental Time Response

Characteristics with the $30 \mathrm{psig}$ Regulated Trailing Edge Opening Test Condition at 0.7

Degrees Angle of Attack with an $82 \mathrm{ft} / \mathrm{s}$ Freestream Velocity ............................................ 144

Figure F. 1: Top Lift Calibration with the Active Circulation Control Model ......................... 155

Figure F. 2: Top Drag Calibration with the Active Circulation Control Model ...................... 155

Figure F. 3: Bottom Lift Calibration with the Active Circulation Control Model .................... 156

Figure F. 4: Bottom Drag Calibration with the Active Circulation Control Model .................. 156

Figure H. 1: Zoomed in view of the 2-D grid near the trailing edge of the model................... 161

Figure H. 2: Zoomed In View of the 2-D grid near the leading edge.................................. 161

Figure I. 1: Streamlines for Trailing Edge Upper (TEU) Blowing at $1200 \mathrm{ft} / \mathrm{s}$...................... 165

Figure I. 2: Streamlines for the Leading Edge Upper (LEU) Blowing at $800 \mathrm{ft} / \mathrm{s}$ case ............. 166

Figure I. 3: Trailing Edge Streamlines for Velocity 150 and LEU600TEU1400 ..................... 166

Figure I. 4: Leading Edge Streamlines for V150LEU600TEU1400 …................................ 167

Figure I. 5: Streamlines for LEL600TEU1400 ................................................................... 167

Figure I. 6: Velocity Magnitude Contour near Trailing Edge for LEL600TEU1400................ 168

Figure I. 7: Trailing Edge Velocity Magnitude Contour for TEU400 ................................... 168

Figure I. 8: Streamlines for TEU400 ............................................................................. 169

Figure I. 9: Trailing Edge Velocity Magnitude Contour for TEU1200 .................................. 169

Figure I. 10: Streamlines for LEU200TEU600 .................................................................... 170

Figure I. 11: Trailing Edge Streamlines for LEU200TEU600 .............................................. 170

Figure I. 12: Streamlines for LEU200 ....................................................................... 171

Figure I. 13:Trailing Edge Streamlines for LEU200 ....................................................... 171

Figure I. 14: Leading Edge Streamlines for LEU200 ....................................................... 172

Figure I. 15: Streamlines for LEU200TEU600.............................................................. 172

Figure I. 16: Trailing Edge Streamlines for LEU200TEU600 .............................................. 173

Figure I. 17: Leading Edge Streamlines for LEU200TEU600 .............................................. 173

Figure I. 18: Drag Response Characteristics for the lower trailing edge at $400 \mathrm{ft} / \mathrm{s}$ and the upper

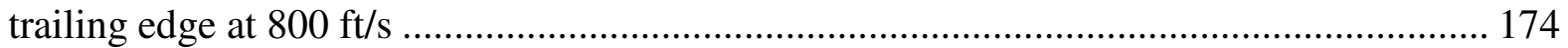

Figure I. 19: Drag Response for LEL600TEU1400.......................................................... 174

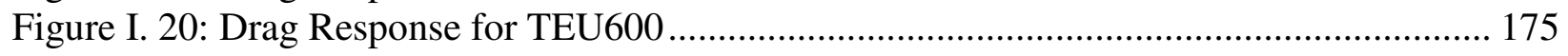

Figure I. 21: Lift Response for TEU600 .......................................................................... 175

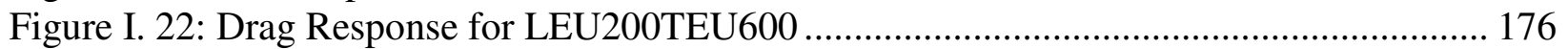

Figure I. 23: Lift Response for LEU200TEU600 ................................................................ 176

Figure I. 24: Lift Response for LEU200 ........................................................................ 177

Figure I. 25: Drag Response for LEU200 ..................................................................... 177

Figure I. 26: Lift Response for LEU200TEU600 .......................................................... 178 
Figure I. 27: Drag Response for LEU200TEU600. 


\section{List of Tables}

Table 4.1: Calculated Centrifugal Acceleration and Corresponding Force on an Air Molecule for

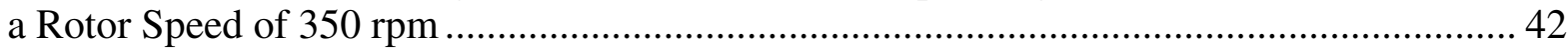

Table 4.2: Lift Coefficient for the NACA 0010-34 Airfoil, Abbott and vonDoenhoff [1959] .... 44

Table 4.3: Drag Coefficient for the NACA 0010-34 Airfoil, Abbott and vonDoenhoff [1959] .. 45

Table 4.4: Summary of the Reynolds Numbers for the Experimentally Tested Velocities and Corresponding Radius at Flight Speeds of $50 \mathrm{mph}$...................................................... 49

Table 4.5: Summary of the Reynolds Numbers from the Computational Study and Corresponding Radius at Flight Speeds of 500 and $100 \mathrm{mph}$............................................ 49

Table 5.1: Surface Pressure Tap Locations, in the Chordwise Direction on the Upper Skin...... 54

Table 5.2: Angle of Attack Matrix Used for Baseline Model Testing........................................69

Table 5.3: Correlation Between Indicated and Geometric Angles of Attack for the Baseline

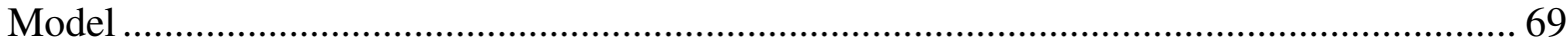

Table 5.4: Steady State Testing Conditions used for Force Augmentation Study ..................... 75

Table 5.5: Test Parameters for the Dynamic Tests .................................................................. 75

Table 6.1: Major parameters used for computational analysis ............................................ 93

Table 6.2: Summary of the data collected for the grid independence study.............................. 94

Table 6.3: Summary of the trailing edge, upper surface blowing computational results as found

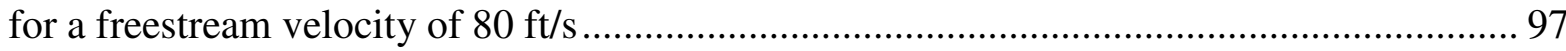

Table 6.4: Summary of the Trailing Edge, Lower Surface Blowing Computational Results as

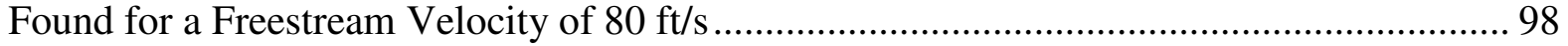

Table 6.5: Summary of Leading Edge Upper Surface Blowing at $C_{\mu \_L E}$ of 0.091 with a Freestream Velocity of $80 \mathrm{ft} / \mathrm{s}$ Cases 102

Table A. 1: Summary of the Blockage Ratios Determined for the NACA 0009 and NACA 0012 Airfoils with a Chordlength of 6 inches.................................................................... 121

Table A. 2: Summary of the Blockage Ratios Determined for the NACA 0009 and NACA 0012 Airfoils with a Chordlength of 9 inches................................................................. 122

Table A. 3: Summary of the Blockage Ratios for 20 inch and 10 inch Chordlenth 10:1 Elliptical Airfoils

Table B. 1: Summary of the Rotational Velocity and Local Reynolds Number for a $15 \mathrm{ft}$ Radius

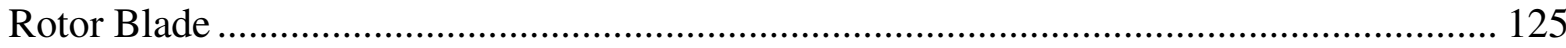

Table E. 1: Lift and Drag Coefficients for the Baseline Model at Various Angles of Attack.... 146

Table E. 2: Lift and Drag Coefficients for the Baseline Model at Various Angles of Attack

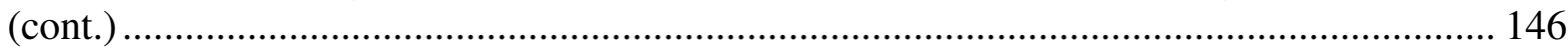

Table E. 3: Baseline Force Data for Run 1, Recorded at $10 \mathrm{kHz}$........................................ 147

Table E. 4: Baseline Force Data for Run 1-A, Recorded at 20 kHz .................................... 147

Table E. 5: Baseline Force Data for Run 2 ........................................................................ 148

Table E. 6: Baseline Force Data for Run 3 .......................................................................... 148

Table E. 7: Baseline Force Data for Run 4 .................................................................... 149

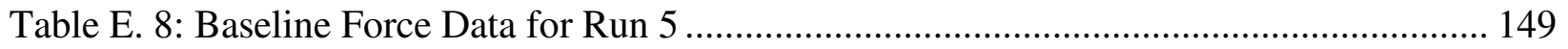

Table E. 9: Baseline Force Data for Run 6 ..................................................................... 150

Table E. 10: Baseline Force Data for Run 7 .................................................................... 150

Table E. 11: Baseline Force Data for Run 8 .................................................................. 151 
Table E. 12: Baseline Force Data for Run 9 .................................................................. 151

Table E. 13: Baseline Force Data for Run 10 .................................................................... 152

Table E. 14: Baseline Force Data for Run 11 .................................................................. 152

Table E. 15: Baseline Force Data for Run 12 ................................................................ 153

Table E. 16: Baseline Force Data for Run 13 ...................................................................... 153

Table G. 1: Summary of Lift and Drag Coefficients Found at 0.68 Degrees Angle of Attack for the Trailing Edge Blowing Slot .................................................................................... 158

Table G. 2: Summary of the Lift and Drag Coefficients at 0.68 Degrees Angle of Attack for the Leading Edge Blowing Slot....

Table G. 3: Summary of the Lift and Drag Coefficients at 0.68 Degrees Angle of Attack and Leading Edge Regulated Pressure at 10 psig....

Table G. 4: Summary of the Lift and Drag Coefficients at 0.68 Degrees Angle of Attack and Leading Edge Regulated Pressure at 20 psig.

Table G. 5: Summary of the Lift and Drag Coefficients at 0.68 Degrees Angle of Attack and Leading Edge Regulated Pressure at 30 psig....

Table I. 1: Summary of the Computational Data with Various Wind Speeds and No Circulation Control Blowing. 163

Table I. 2: Summary of the Computational Data with Various Wind Speeds and an Upper Surface Trailing Edge Blowing of $1400 \mathrm{ft} / \mathrm{s}$ 163

Table I. 3: Summary of the Computational Data with Various Wind Speeds, Upper Surface Leading Edge Blowing of $600 \mathrm{ft} / \mathrm{s}$ and Trailing Edge Jet Velocity of $1400 \mathrm{ft} / \mathrm{s}$ 


\section{Nomenclature}

\begin{tabular}{|c|c|c|}
\hline$\underline{\text { Symbol }}$ & $\underline{\text { Description }}$ & $\underline{\text { Units }}$ \\
\hline \multicolumn{3}{|c|}{ English } \\
\hline $\mathrm{A}$ & Model Frontal Area, Constant & in $^{2},-$ \\
\hline a & Radius of Curvature, acceleration & inches, $\mathrm{ft} / \mathrm{sec}^{2}$ \\
\hline $\mathrm{B}$ & Constant & - \\
\hline $\mathrm{b}$ & Slot Height, Jet Half-Width & inches, inches \\
\hline $\mathrm{C}$ & Constant & - \\
\hline $\mathrm{c}$ & Chordlength & inches \\
\hline $\mathrm{C}_{\mathrm{do}}$ & Coefficient of Drag & - \\
\hline $\mathrm{C}_{1}$ & Coefficient of Lift & - \\
\hline $\mathrm{C}_{\mathrm{Q}}$ & Volume Flow Coefficient & - \\
\hline $\mathrm{C}_{\mu}$ & Blowing Coefficient & - \\
\hline $\mathrm{CC}$ & Circulation Control & - \\
\hline $\mathrm{CCHB}$ & Circulation Control Helicopter Blade & - \\
\hline $\mathrm{E}$ & Empirical Separation/Entrainment Factor & - \\
\hline $\mathrm{F}$ & Boundary Layer Function & - \\
\hline $\mathrm{H}$ & Thwaites Shape Factor & - \\
\hline I & Integral Parameter & - \\
\hline $\mathrm{K}$ & Entrainment Correction Factor & - \\
\hline $\mathrm{k}$ & Constant & 0.8814 \\
\hline $\mathrm{L}$ & Lift Force & $\mathrm{lbf}$ \\
\hline LE & Leading Edge & - \\
\hline LEL & Leading Edge Lower Surface & - \\
\hline LEU & Leading Edge Upper Surface & - \\
\hline M & Segment of Airfoil Surface, Dimensionless Mass Flux, Mach Number &,,--- \\
\hline$\dot{m}$ & Mass Flow Rate & slug/sec \\
\hline $\mathrm{n}$ & Normal Direction & - \\
\hline $\mathrm{n}$ & Constant & $6<\mathrm{n}<7$ \\
\hline $\mathrm{P}$ & Pressure, Plenum Pressure & psf, psf \\
\hline q & Dynamic Pressure & psf \\
\hline
\end{tabular}


r

$\operatorname{Re}$

Ri
Distance Along Surface, Radius of Curvature, Radius of Rotor

Reynolds Number

Richardson Number

Thwaites Shear Correlation, Test Section Cross-Sectional Area

Location Along Surface

Temperature

Time, Tangent to.., Slot Thickness

Trailing Edge

Trailing Edge Lower Surface

Trailing Edge Upper Surface

$\mathrm{X}$-Direction Velocity Velocity

Y-Direction Velocity

Fluctuation in the X-Direction

Distance

Height above surface

Spalding Velocity Parameter

Thwaites Parameter

Symbol

$\begin{array}{ccc}\alpha & \text { Angle of Attack } & \text { deg } \\ \beta & \text { Constant } & 0.66 \\ \frac{\partial}{\partial s} & \text { Partial Derivative in Surface Direction } & - \\ \frac{\partial}{\partial x} & \text { Partial Derivative in X-Direction } & - \\ \frac{\partial}{\partial y} & \text { Partial Derivative in Y-Direction } & - \\ \delta & \text { Boundary Layer Displacement Thickness } & \text { inches } \\ \Delta \mathrm{C}_{\mathrm{do}} & \text { Measured Drag Coefficient } & \\ \varphi & \text { Potential of Motion Around Airfoil } & \end{array}$


$\underline{\text { Symbol }}$

$\Phi$

$\phi$

$\Gamma$

$\gamma$

$\Lambda$

$\lambda$

$\mu$

$v$

$\theta$

$\rho$

$\tau_{\mathrm{xx}}$

$\tau_{\mathrm{yy}}$

$\tau_{\mathrm{xy}}$

$\omega$

$\psi$

$\xi$
Location on Mapped Circular Path

Circulation

Vortex Strength, Ratio of Specific Heats

Karman-Pohlhausen Parameter, Sweep Angle

Thwaites Parameter

Viscosity, Advance Ratio

Kinematic Viscosity

Angular Position, Boundary Layer Momentum Thickness, Location of Flow Separation

Density

Normal Stress in X-Direction

Normal Stress in Y-Direction

XY Shear Stress

Frequency, Rotational Speed

Azimuth Location

Angle Between Coordinate and Jet Exit $\underline{\text { Units }}$

radians

-, deg

slug/ft-s, -

$\mathrm{ft}^{2} / \mathrm{sec}$

deg, inches, deg

$\operatorname{slugs} / \mathrm{ft}^{3}$

$\mathrm{lbf} / \mathrm{ft}^{2}$

$\mathrm{lbf} / \mathrm{ft}^{2}$

$\mathrm{lbf} / \mathrm{ft}^{2}$

$\mathrm{Hz}, \mathrm{rad} / \mathrm{s}$

deg

deg

\section{Subscripts}

\begin{tabular}{ccc}
\hline 1 & X-Direction, Lower Surface & - \\
2 & Upper Surface & - \\
comp & Compressor & - \\
$\mathrm{d}$ & Plenum & - \\
$\mathrm{e}$ & Effective & - \\
$\mathrm{exp}$ & Experimental & - \\
$\mathrm{i}$ & Index Direction & - \\
$\mathrm{j}$ & Jet & - \\
$\mathrm{m} / 2$ & Half-Width of Jet & - \\
max & Maximum & - \\
meas & Measured & - \\
norm & Normal & - \\
o & Plenum &
\end{tabular}


$\underline{\text { Symbol }}$

$\mathrm{P}$

$r$

S

surf

W

$\infty$

$\wedge$

$-, \rightarrow$
Description

\section{$\underline{\text { Units }}$}

Pressure

Radial Direction

Parallel to Surface

Surface

Wall

Freestream, Atmospheric

\section{Superscripts}

Fluctuating Value, Per Unit Span

Turbulent Fluctuations

Vector

Vector 


\section{Acknowledgements}

The author would like to extend his appreciation to his dissertation committee for their interest and assistance. The author would especially like to thank Dr. James Smith for his guidance and support during this research effort. In addition to his committee members the author was given support from the various members of the Center for Industrial Research Applications. The author would like to thank Mr. Jay Wilhelm, Mr. Justin Smith, and Mr. Franz Pertl for their assistance with the data acquisition and valve actuation systems.

The author would also like to thank Mr. Jon Kweder, Mr. Steve Hard, Mr. Andrew Reeder, Mr. Joe Allen, Mr. D. Scott Loud, Mr. Chad Panther, Mr. Pat Wildfire, Ms. Meagan Hubbell, Mr. John Ruth, Mr. Henry Graham and Mrs. Emily Pertl for their assistance in the collection of data for this research.

The author would be remiss if thanks were not given to his extended family for their unwavering support. 


\section{Introduction}

Helicopters are rotary wing aircraft that are more flexible in their flight envelope than fixed wing aircraft. Helicopters have the ability to hover in place, take off and land vertically, as well as fly in any orientation of the aircrafts' body relative to the direction of motion. The main forces on any aircraft, including helicopters, are weight, lift, drag and thrust. In helicopters the main rotor provides both the lift and the thrust, depending on its attitude with the horizon; i.e., tilting the rotor forward provides thrust while maintaining total lift, which is augmented by the lift generated by the rotor 'disc' as air flows over it similar to a traditional fixed wing.

Forward flight and natural winds create asymmetry due to the relative motion of the ascending (advancing) and descending (retreating) blades to the air. The ascending blade experiences a gain in lift due to the increased relative velocity of the air due to the forward motion of the entire aircraft. Similarly, the descending blade encounters a decrease in relative speed and a resulting lift deficit. To counteract this effect, helicopter designers alter the angle of attack, or pitch, of the blade as it travels around in the plane of the rotor, adding lift to the retreating blade by increasing the root angle-of-attack while decreasing the pitch on the advancing blade. It is important to state that all blades of a helicopter, cycle from ascending to descending once every complete rotation of the rotor assembly.

The most complex and maintenance intensive component of a helicopter is the swash-plate. This part alters the pitch of the main rotor blades, as they rotate through their path, to compensate for the asymmetry of lift as well as provide pitch and roll control to the aircraft. The swash-plate connects to the blades at their root and is controlled via pilot input for both collective and cyclic pitch control. The collective rotor control adjusts the rotor blades equally, changing the total lift being generated to provide vertical movement of the craft (ascent or decent). The alternating pitch of the blade is controlled by the cyclic controls of the rotor to create turning and accelerating forces. The mechanical nature of these motions requires constant inspection, maintenance of the swash-plate, and is a major issue for the safety of the rotorcraft. With the advances in computing power and fly-by-wire technology for fixed wing aircraft it is believed that 
helicopters can also be improved using high lift technologies. These technologies could be combined with aspects of flow and circulation control (CC), such as inducing the Coanda Effect, to aerodynamically change the performance characteristics of the rotor without requiring a physical change in the geometry.

The use of circulation control on an airfoil can significantly change its lift performance. The utilization of this enhanced lift coefficient can increase the potential payload capacity of a specific rotor blade, resulting in the ability to either carry larger payloads or operate at slower rotor speeds. It is also likely that cycling the circulation control parameters (exit velocity) can result in the ability to account for the asymmetry of lift, allowing for the reduction in requirements for the swash-plate and its potential removal from the helicopter. To determine the feasibility of replacing the swash-plate with a scheduled circulation control system, two key parameters need to be determined; the amount of lift increase by circulation control, and the time scale at which the force augmentation is achieved.

Active circulation control techniques have previously been applied to airfoils with moderate thickness ( $20 \%$ chord length). Airfoils of this thickness are rarely used in a helicopter main rotor blade, thus for this testing program a thinner airfoil profile, a $10 \%$ thick airfoil, was chosen. A symmetric, elliptical airfoil was selected to provide the most flexibility in the test matrix. Creating the symmetric circulation control system offers the ability to investigate the capability to increase and decrease the lift coefficient. Alternating the lift augmentation enables the flexibility to set the angle of attack at a mean value and cyclically alter (increase/decrease) the lift forces to duplicate the change in pitch angle currently achieved with the swash-plate.

For the initial testing in this program, an elliptic airfoil was used for the Circulation Control Helicopter Blade (CCHB). The elliptic profile allows for the addition of blowing slots near the trailing edge to induce the Coanda Effect over the rounded trailing edge. Due to the rotary movement of the blades, the ability to alter the circulation control around the path of the blade is desired. The use of multiple blowing slots on the upper and/or lower surface(s) provides the greatest flexibility in the resulting aerodynamic force coefficients due to the ability to both increase and decrease the values of lift and drag coefficient. By adjusting the blowing coefficient, selecting which slot is 
open, the lift can be augmented to reduce the asymmetry of lift in the helicopter rotor created by maneuvering flight. The lift augmentation could potentially be enhanced by a rotating slot at the leading edge of the airfoil to aerodynamically simulate a cambered airfoil. Another potential benefit of a slot near the leading edge would be the ability to alter the pitching moment of the blade, which would allow designers to reduce the stresses in the blade itself.

Prior research in this area, discussed further in the literature review, controlled the circulation control blowing by altering the plenum pressures at the hub of the rotor. The current research project is the initial proof of concept testing of a high lift rotor blade using slots that are controlled at the surface. Two characteristics of the Circulation Control Helicopter Blade which need to be proven during this investigation are the achievable change in lift and determining the time in which this lift change can occur. The minimum acceptable requirement for the lift change would be that which corresponds to 5 to 8 degree angle of attack change which is used currently with conventional rotors. Most conventional rotors range in speed from 250 to $350 \mathrm{rpm}$; the goal of the CCHB would be to maintain the current rotor speed. Assuming the use of four control points at 90 degree intervals (i.e. fore, aft, left, and right) this would set the target response time of the lift augmentation at $60 \mathrm{msec}(250 \mathrm{rpm})$ to $43 \mathrm{msec}(350 \mathrm{rpm})$ for the free stream response to the introduction of the circulation control blowing jets. 


\section{Research Objectives}

The primary research objective of this project is to determine the response time of the aerodynamic forces from a circulation control airfoil, with localized, near-surfaceactuated blowing slots. The associated time-dependent change in force (lift and drag) of the airfoil shape will be reported, along with the various measured response times. The relationship between the force augmentation and the response time is expected to be the critical trade-off to be made for the application of circulation control to the main rotor of a helicopter. Another objective of the research is to computationally investigate the effects of azimuth location on the effectiveness of circulation control.

Both computational and experimental techniques were used to investigate this concept. The results of the two methods have been compared to historical lift augmentation capabilities developed primarily for fixed wing aircraft. The time response characteristics of the slot controlled circulation control were also determined. A prediction of the blade's span-wise lift distribution and rotor speed limitations of the CCHB concept was made based on the results of the two analysis methods. 


\section{Literature Review}

This chapter summarizes the flow physics of circulation control and prior research into blowing jets and circulation control. This summary starts with the basic fluid flow relationships, and then presents early applications of circulation control including the Reversed Blowing Circulation Control Rotor. Some of the topics discussed include boundary layer profiles, flow entrainment and separation, circulation around an airfoil and factors that influence the performance of a circulation control airfoil.

\subsection{Basic Fluid Flow Relationships}

The two-dimensional incompressible flow conservation equations stated by Ambrosiani [1971] as shown in Equation 3.1, Equation 3.2, and Equation 3.3 which are the continuity equation and the $\mathrm{x}$ and $\mathrm{y}$ conservation of momentum relationships, respectively, written in thin shear layer form, to show effects of flow geometry radius of curvature. Equation 3.4, Equation 3.5, and Equation 3.6 define the normal $\left(\tau_{\mathrm{xx}}\right.$ and $\left.\tau_{\mathrm{yy}}\right)$ and shear $\left(\tau_{\mathrm{xy}}\right)$ stress terms used in the conservation equations. These relationships are functions of the density, $\rho$, viscosity, $\mu$, time, $t$, the surface radius of curvature, $\mathrm{R}$, height above the surface, $\mathrm{y}$, and the $\mathrm{x}$ and $\mathrm{y}$ velocities, $\mathrm{u}$ and $\mathrm{v}$, respectively:

$$
\begin{aligned}
& \frac{\partial \rho}{\partial t}+\frac{R}{R+y} \frac{\partial(\rho u)}{\partial x}+\frac{R}{R+y} \frac{\partial\left(\frac{R+y}{R}(\rho v)\right)}{\partial y}=0, \quad \text { Equation } 3.1 \\
& \rho\left[\frac{\partial u}{\partial t}+\frac{R}{R+y} u \frac{\partial u}{\partial x}+v \frac{\partial u}{\partial y}+\frac{u v}{R+y}\right]+\frac{R}{R+y} \frac{\partial P}{\partial x} \\
& -\frac{R}{R+y} \frac{\partial \tau_{x x}}{\partial x}-\frac{\partial \tau_{x y}}{\partial y}-\frac{2}{R+y} \tau_{x y}=0, \\
& \rho\left[\frac{\partial v}{\partial t}+\frac{R}{R+y} u \frac{\partial v}{\partial x}+v \frac{\partial v}{\partial y}-\frac{u^{2}}{R+y}\right]+\frac{\partial P}{\partial y} \\
& -\frac{R}{R+y} \frac{\partial \tau_{x y}}{\partial x}-\frac{\partial \tau_{y y}}{\partial y}-\frac{1}{R+y}\left[\tau_{y y}-\tau_{x x}\right]=0
\end{aligned}
$$




$$
\begin{array}{ccc}
\tau_{x x}=2 \mu\left[\frac{R}{R+y} \frac{\partial u}{\partial x}+\frac{v}{R+y}-\frac{1}{3}\left(\frac{R}{R+y} \frac{\partial u}{\partial x}+\frac{\partial v}{\partial y}+\frac{v}{R+y}\right)\right], & \text { Equation } 3.4 \\
\tau_{y y}=2 \mu\left[\frac{\partial v}{\partial y}-\frac{1}{3}\left(\frac{R}{R+y} \frac{\partial u}{\partial x}+\frac{\partial v}{\partial y}+\frac{v}{R+y}\right)\right], & \text { Equation } 3.5 \\
\tau_{x y}=\mu\left[\frac{R}{R+y} \frac{\partial v}{\partial x}+\frac{R+y}{R} \frac{\partial\left(\frac{R}{R+y} u\right)}{\partial y}\right] . & \text { Equation } 3.6
\end{array}
$$

\subsection{Jet Velocity Profiles}

The first reported use of blowing slots to control lift is attributed to Hagedorn and Ruden in 1938, who noticed an unaccountable increase in lift at high blowing rates during investigations into boundary layer control on a flap, see Wilson [1973]. Interest in active blowing systems increased upon the arrival of the turbojet engine, initially in Great Britain and France on a jet flap configuration. The flow of a fluid over curved surfaces has since been studied for a variety of applications. The research activities highlighted within this summary are those focused on lift augmentation methods in particular the flow phenomenon known as the Coanda effect.

Starting in the late 1960's, circulation control was investigated for various applications including but not limited to: fixed wing aircraft, rotary wing aircraft, X-wing aircraft and submarines. The basic concept of circulation control was to alter the circulatory flow around an object to affect the forces generated, particularly lift, and drag. The most commonly used technique to achieve circulation control was to use a thin jet, blown over a rounded trailing edge which induces the Coanda Effect, altering the lift and drag.

Newman [1961] discusses the expansion of a free jet, as shown in Figure 3.1 as dependent upon the location from the origin of the jet. By placing a jet near a surface a wall jet results, as illustrated in Figure 3.2, the presence of the wall alters the pressure and 
velocity profiles on the jet. Figure 3.3 shows that the difference in pressure that alters the flow near a wall, when placed in the presence of an inclined wall results in the reattachment of the flow, turning the jet and altering the streamlines of flow in the proximity of the jet.

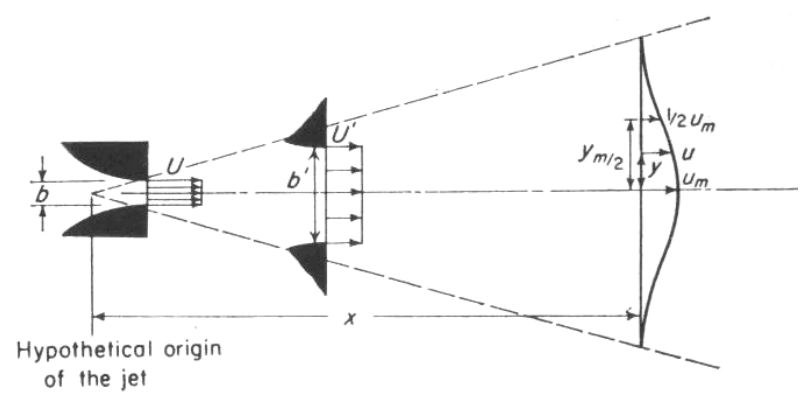

Figure 3.1: Two-dimensional free turbulent jet, Newman [1961]

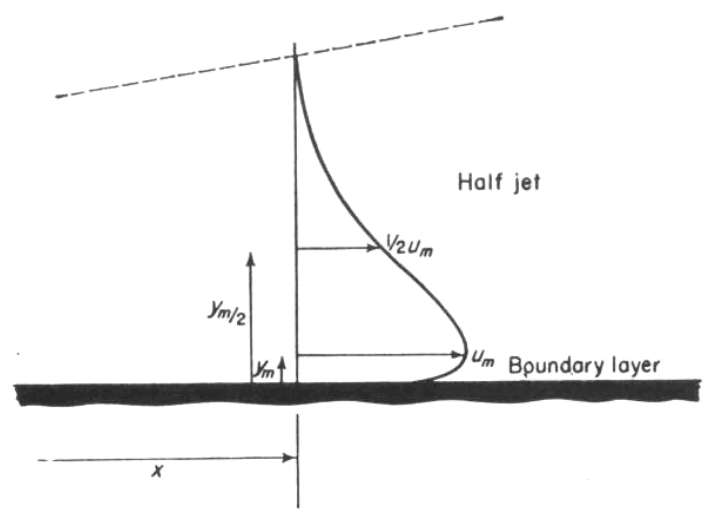

Figure 3.2: Two-dimensional turbulent wall jet, Newman [1961]

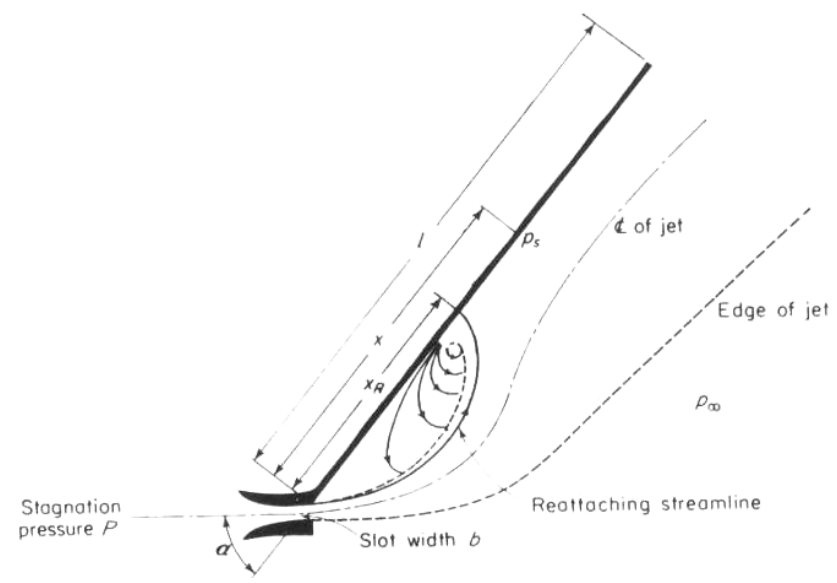

Figure 3.3: Re-attachment of a two-dimensional jet to an inclined flat plate, Newman [1961] 
Ramaprian and Shivaprasad [1977, 1978] investigated the influence of curved surfaces on boundary layers, and concluded that a convex surface has significant impact on the structure of a turbulent boundary layer particularly increasing the wake of the outer portion of the boundary layer. This increase in wake was presumed to be caused by the centrifugal forces. The curvature of the surface also tends to increase the shear stress in the outer portion of the jet, while decreasing it in the inner portion, Gibbs [1975].

Additional experimental studies have been conducted with jets in the proximity of a curved surface. Johnson [1975] conducted tests on a two-dimensional hyper-mixing curved jet, using two jet velocities (4.6 and 7.28 times the free stream speed). Loth, et al. [1976] found that for cases where the momentum within the jet is sufficiently higher (3 or 4 times) than the momentum in the free stream, the inflow velocities do not differ significantly from the static case. Newman [1961] also found that the curved wall jet flow is not influenced by Reynolds number, based on the blowing slot value, provided it is above a value of 9,000. Above these thresholds the jet is the governing mechanism of the flow, independent of the free stream conditions [Churchill, 1992].

The more complex flow scenario of a wall jet adjacent to a curved boundary surface is the deflection of the jet by the boundary, which is referred to as the Coanda effect [Newman, 1961]. This effect is used to refer to cases where flow travels over the top of a convex surface that recedes away from the streamline [Churchill, 1992]. Ambrosiani [1971] defines the Coanda Effect as the deflection of a plane wall jet by its adjacent boundary. This deflection is created by the balance of the centrifugal force in the jet and the reduced pressure at the wall, caused by the jet velocity [Englar, 1975]. Using a rounded trailing edge enables the rear stagnation point to be forced below the chord line thus altering the circulation around the airfoil, consequently increasing the lift.

A common example of the Coanda effect is to place one's finger under a running faucet of water. As the curved edge of the finger makes contact with the edge of the stream of water the entire stream is altered to deflect around the finger. The phenomenon is commonly attributed to Henri-Marie Coanda who, according to Churchill [1992], invented and tried to market several applications of the concept [Debus, 1968]. 
The Coanda effect is physically characterized by a balance of inertial and pressure forces [Churchill, 1992]. The wall jet energizes the boundary layer creating a lower pressure near the surface than in the free stream which delays the separation of the free stream from the surface. As the wall jet thickens from the additional entrained mass, from the free stream and viscous dissipation, the pressure at the wall increases until the pressures (surface and free stream) equalize at which point jet separation typically occurs [Ambrosiani, 1971]. Ambrosiani also states that the location of the separation point is dependent upon the blowing rate.

Churchill [1992] offers an alternative description of the Coanda effect as the entrainment of fluid from both sides of the jet, which due to the limited mass near the wall, the pressure is reduced. This lower pressure in turn pulls the jet toward the surface, providing a pressure gradient force to resist the jet's inertial resistance to turning. As the jet travels downstream, viscosity reduces the thrust within the jet allowing the pressure to rise until an adverse pressure gradient occurs and the flow separates from the surface.

Once the use of jet flaps and other blown circulation augmentation were found beneficial, a means of theoretically predicting the flow field was sought. Many early methods were empirical in nature; see Wilson [1973]. The high velocity jet flap and blowing slots located near the trailing edge of the airfoil results in super-circulation, which Wilson defined as circulation greater than that possible for the unaltered airfoil. Stratford [1959] suggested the use of ejector thrust augmentation to reduce the required jet exit velocity and found that the ejector further increases the direct lift force as compared with the jet exit alone.

\subsection{Boundary Layer Profiles}

The boundary layer profile shapes predicted by Newman [1961] described the distance from the surface at which the velocity is half the maximum, $\mathrm{y}_{\mathrm{m} / 2}$, and the surface pressure, $\mathrm{P}_{\text {surf }}$, distribution are shown in Equation 3.7, and Equation 3.8, respectively:

$$
\frac{y_{m / 2}}{r \theta}=0.11\left(1+1.5 \frac{y_{m / 2}}{r}\right),
$$


and

$$
\frac{\left(P_{\infty}-P_{\text {surf }}\right) r}{\left(P-P_{\infty}\right) b}=\frac{8 \ln \left(1+\frac{4 y_{m / 2}}{3 r}\right)}{\left(\frac{4 y_{m / 2}}{3 r}+1\right)^{2}-1+2 \ln \left(1+\frac{4 y_{m / 2}}{3 r}\right)} . \quad \text { Equation } 3.8
$$

These relationships are two dimensional, per unit depth, and depend on the radius of curvature, $\mathrm{r}$, angular position from the slot exit, $\theta$, the slot height, $\mathrm{b}$, free-stream pressure, $\mathrm{P}_{\infty}$, and the slot plenum pressure, $\mathrm{P}$. Newman also approximated the separation location of the jet over a cylinder as shown in Equation 3.9, which has been based on a limiting value of 245 degrees, which agrees with theory and experiment, according to Newman [1961].

$$
\theta_{\text {sep }}=245-\frac{391 \frac{b}{r}}{\left(1+1.125 \frac{b}{r}\right)}
$$

Equation 3.9

The profile of $\mathrm{y}_{\mathrm{m} / 2}$ from Newman was improved upon by Wilson and Goldstein [1976], as shown in Equation 3.10.

$$
\frac{y_{m / 2}}{b}=0.0787\left(\frac{r \theta}{b}+6\right)\left[1+2.956\left(\frac{y_{m / 2}}{r}\right)-0.1559\left(\frac{y_{m / 2}}{r}\right)^{2}\right]
$$

The authors also provide the velocity profile shown in Equation 3.11 for the jet along the curved surface. Looking at the boundary layer profile of a jet blown tangent to a circular cylinder in stagnate surroundings, as illustrated in Figure 3.4 from Newman [1961], shows the tendency of the jet to deflect with the surface.

$$
\frac{V_{\text {jet }}}{U_{m}}=0.0359\left(\frac{r \theta}{b}+6\right)^{1.05}\left[1+3.354\left(\frac{y_{m / 2}}{b}\right)\right]
$$

Figure 3.5, from Gibbs [1975], shows the boundary layer development in the proximity of a convex surface. From this figure it is seen that the momentum of the jet drastically changes the boundary layer profile of the free-stream flow over the surface. Myer [1972] states that blowing high energy air tangentially into the boundary layer near the trailing edge of an airfoil introduces kinetic energy (or momentum as described by 
Bauman [1976]) into the boundary layer and produces a velocity profile which has been experimentally shown to delay flow separation. Examining the boundary layer profile and jet interaction further around a semi-circular airfoil trailing edge, as shown in Figure 3.6, from Myer, the upper surface separation point can be moved to the lower surface of the airfoil.

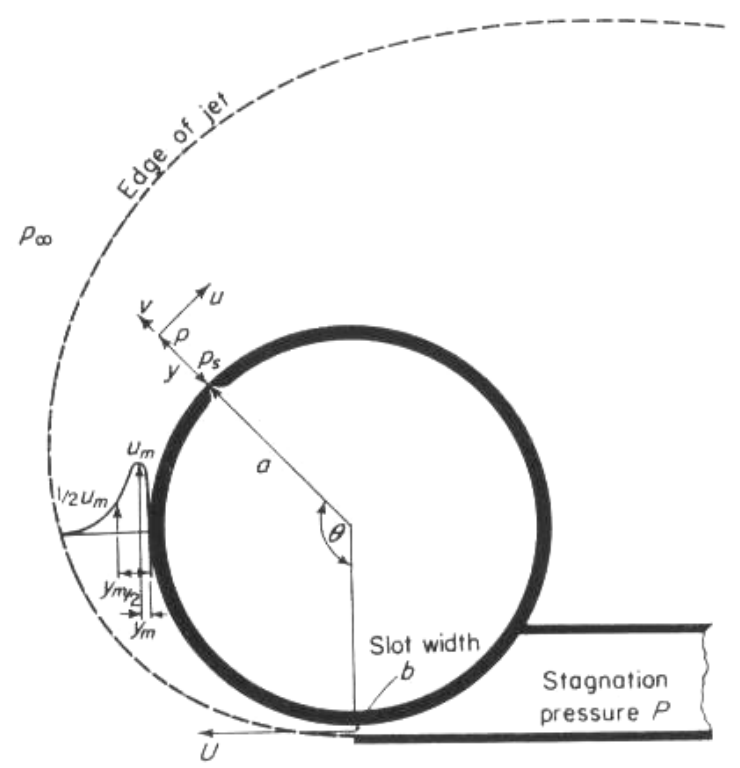

Figure 3.4: Flow of a two-dimensional jet round a circular cylinder, Newman [1961]

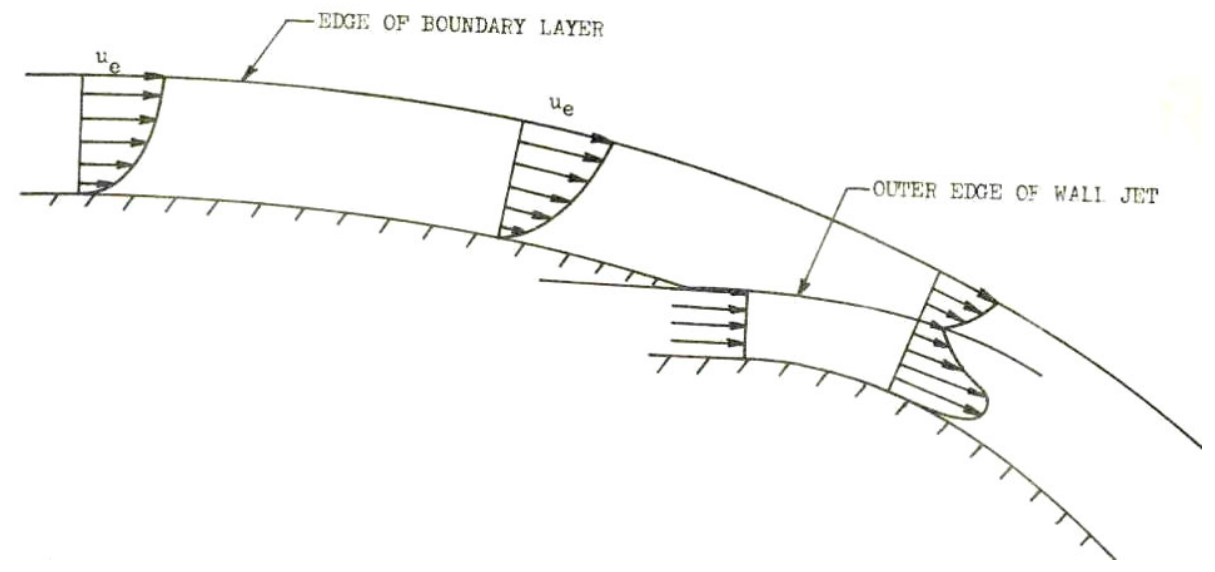

Figure 3.5: Boundary layer development of a wall jet, Gibbs [1975] 


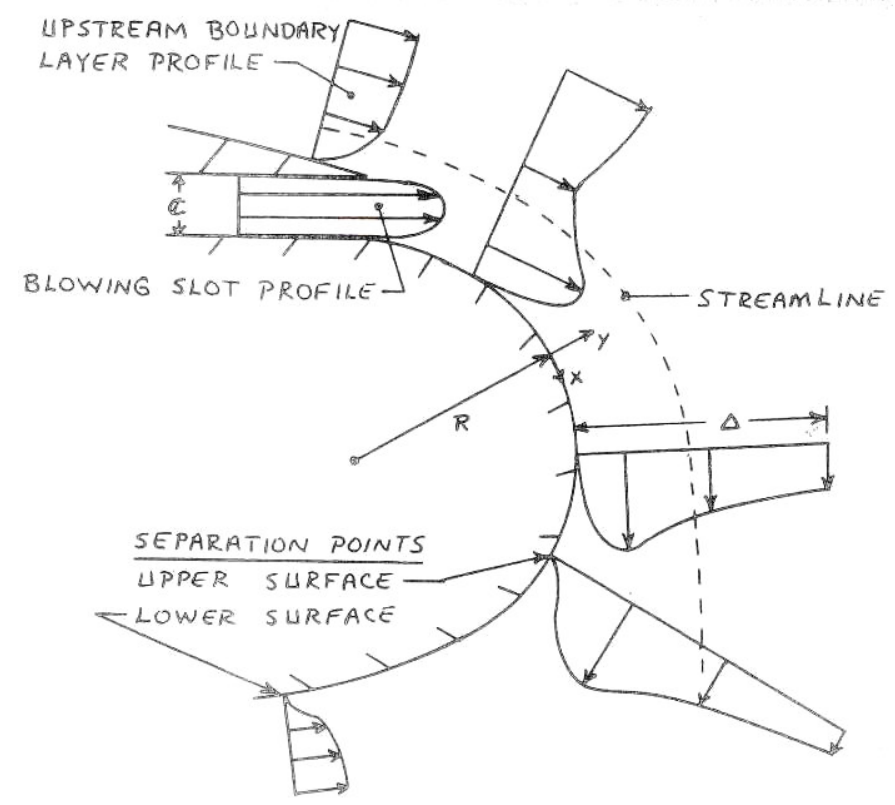

Figure 3.6: Boundary layer-jet interaction over a Coanda surface, Myer [1972]

According to Wilson [1973], the governing equation for incompressible, potential flow is the continuity equation, Equation 3.12. The author also states that the Fredholm integral equation of the first kind, Equation 3.13, does not result in a well behaved set of equations for wing sections with a blowing element (jet flap, blowing slot). However, a well behaved numerical set of equations can be found from a Fredholm equation of the second kind, Equation 3.14, where, $\Phi$ is the total velocity potential, $\alpha_{t}$ is the airfoils angle of attack, $\mathrm{r}_{\mathrm{PQ}}$ is the distance between coordinate on segment $\mathrm{M}$ and the midpoint of another segment, $\gamma$ is the strength of the vortex distribution, $\hat{n}$ is the unit vector normal to the surface, $\vec{V}_{\infty}$ is the free-stream velocity vector, $\hat{t}$ is the unit vector tangent to the surface, s is the coordinate along the body surface, and $\xi$ is the angle between the coordinate s and the exit of jet.

$$
\begin{gathered}
\nabla^{2} \Phi=0 \\
\frac{1}{2 \pi} \oint \frac{\cos \left(\alpha_{t}\right)}{r_{P Q}}(s, \xi) \gamma(\xi) d \xi=-\hat{n} \bullet \vec{V}_{\infty}, \\
\text { and }
\end{gathered}
$$




$$
\frac{1}{2} \gamma(s)+\frac{1}{2 \pi} \oint \frac{\cos \left(\alpha_{t}\right)}{r_{P Q}}(s, \xi) \gamma(\xi) d \xi=\hat{t} \bullet \vec{V}_{\infty} . \quad \text { Equation } 3.14
$$

The key to the potential flow method used by Wilson [1973] is the approximation of the body surface by straight line segments over which the velocity discontinuity distribution is a known and is a function of the surface distance. The author also treated the jet as an extension of the solid structure which has an unknown shape, which was determined through an iterative process which compared the net local pressure force with the centrifugal force acting on the fluid.

A considerable indirect effect of large potential flow velocity and pressure gradients over a small radius of curvature is that an adverse pressure gradient is present. According to Gibbs [1975] the wall jet is rapidly thickened, making the curvature of the streamline outside of the jet greater than the sum of the surface curvature and boundary layer growth.

\subsection{Flow Entrainment}

The entrainment function for a flat plate was modified by adding a correction factor to correct the entrainment rate for a curved surface. Churchill [1992] defined the correction factor, $\mathrm{K}$, as shown in Equation 3.15 which is dependent on the radius of curvature, $r$, and the height above the surface where the velocity is half of the peak jet velocity, $\mathrm{y}_{\mathrm{m} / 2}$. Equation 3.16 is the relationship for the entrainment function for a curved surface based on the dimensionless mass flux in the stream wise direction, $\mathrm{dM} / \mathrm{dx}$. Churchill worked with non-dimensional forms of the equations to eliminate difficulties associated with defining all of the parameters, particularly defining the geometry and other flow properties with consistent units. Non-dimensionalization also makes studies based on conventional dimensionless flow parameters (Reynolds number) achievable with simplified equations. It is important to note that despite the ease of use with dimensionless forms of equations, care should be taken to ensure true dimensionless terms and that the same unit system is used for both the numerator and denominator of the dimensionless term. 


$$
\begin{gathered}
K=\frac{\left[1+4.48\left(\frac{y_{m / 2}}{r}\right)-1.34\left(\frac{y_{m / 2}}{r}\right)^{2}\right]^{2}}{1+1.34\left(\frac{y_{m / 2}}{r}\right)^{2}}\left[\frac{1}{1+0.3\left(\frac{y_{m / 2}}{r}\right)}\right] \\
\text { and } \\
\frac{d M}{d x}=\frac{U}{c U_{\infty}} K(0.03 Z-0.02)
\end{gathered}
$$

An expression for the spreading rate, $\frac{\partial b}{\partial s}$, of a curved wall jet as shown below, was defined by Roberts [1986]. The jet spreading rate is dependent upon the half-width of the jet, $\mathrm{b}$, radius of the surface, $\mathrm{R}$, location along the surface, $\mathrm{s}$, where the constant $\mathrm{k}$ is equal to 0.8814, $\mathrm{n}$ has a value between 6 and 7 based from existing experimental data, and $\mathrm{K}$ is determined experimentally. Thus, when the wall shape is known this relationship, Equation 3.17, can be integrated with respect to surface location to determine the jet half width, $b(s)$.

$$
\frac{\partial b}{\partial s}=K\left[1+\frac{n}{k} \frac{b}{R}\right]+\left(\frac{19}{12}-\frac{\log (2)}{k}\right)\left(\frac{b}{R} \frac{\partial R}{\partial s}\right) \frac{b}{R}+\vartheta\left(\frac{b}{R}\right)^{2}
$$

\subsection{Separation Location Prediction}

According to Harness [1970] the application of Thwaites condition for flow over a bluff ended body requires the circulation around the body to have a value such that the velocity outside of the upper and lower surfaces of the body is the same at the separation locations. This constraint provides a method to predict flow separation. Stratford [1959] defined the separation point of flow over an immersed body based on the pressure coefficient, $\mathrm{C}_{\mathrm{p}}$, and the Reynolds number, Re. The relationship between pressure coefficient and Reynolds number is shown in Equation 3.18, which can be simplified to the criteria shown in Equation 3.19 outside of which the flow is separated. The term $n$ is defined as shown in Equation 3.20 based on the free-stream Reynolds number found from 
Equation 3.21, based on the distance parallel to the surface, $\mathrm{x}_{\mathrm{s}}$, free-stream velocity, $\mathrm{U}_{\infty}$, and the kinematic viscosity, $v$. The term $\beta$ has a value of 0.66 and is constant.

$$
\begin{gathered}
\left(2 C_{p}\right)^{0.25(n-2)}\left(x \frac{d C_{p}}{d x}\right)^{0.5}=1.06 \beta\left(10^{-6} \mathrm{Re}\right)^{0.1} \\
C_{p} \leq \frac{n-2}{n+1} \\
n=\log _{10}\left(\operatorname{Re}_{s}\right) \\
\text { and } \\
\operatorname{Re}_{s}=\frac{x_{s} U_{\infty}}{v} .
\end{gathered}
$$

Gibbs [1975] used Thwaites condition of constant pressure in the separated region to predict the location of flow separation. Thwaites parameters, $\mathrm{S}$, and $\mathrm{H}$, are described by White [1991] as the shear correlation, Equation 3.22, and shape factor of the object within the fluid flow, Equation 3.23. These relationships are defined in terms of shear stress at the wall, $\tau_{\mathrm{w}}$, momentum thickness, $\theta$, fluid viscosity, $\mu, \mathrm{X}$-direction velocity, u, displacement thickness, $\delta^{*}$, and the parameters of $\lambda$ and $\mathrm{z}$ as defined in Equation 3.24 and Equation 3.25, where $\Lambda$ is the Karman-Pohlhausen parameter.

$$
\begin{gathered}
S(\lambda)=\frac{\tau_{w} \theta}{\mu u} \cong(\lambda+0.09)^{0.62}, \\
\text { and } \\
H=\frac{\delta^{*}}{\theta} \cong 2.0+4.14 z-83.5 z^{2}+854 z^{3}-3337 z^{4}+4576 z^{5}, \\
\text { where } \\
\lambda=\frac{\theta^{2} U^{\prime}}{v}=\left(\frac{\theta}{\delta}\right)^{2} \Lambda, \\
\text { and } \\
z=(0.25-\lambda)
\end{gathered}
$$


The Karman Pohlhausen parameter is defined by White [1991] as shown in Equation 3.26 as a function of the boundary layer displacement thickness, $\delta$, pressure, $\mathrm{P}$, and the free-stream velocity, $\mathrm{U}$.

$$
\Lambda=\frac{\delta^{2}\left(\frac{d P}{d x}\right)}{\mu U}
$$

White [1991] also defines a boundary layer function, F, Equation 3.27, which was simplified to a linear fit and flow separation was predicted when $\mathrm{F}$ has a value of 1 , or $\lambda$ is approximately -0.09 .

$$
F(\lambda)=2[S(\lambda)-\lambda(2+H)] \cong 0.45-6.0 \lambda
$$

Equation 3.27

Young [1989] provides yet another description of the onset of flow separation as when the Thwaites parameter $S=0$. The linear fit of Thwaites method is approximately $\pm 5 \%$ for the favorable and mildly adverse pressure gradients and up to $\pm 15 \%$ near flow separation [White, 1991]. The separation point of flow over a cylinder is defined as the point at which the surface shear force is equal to zero by Churchill [1992] and described by the criteria shown in Equation 3.28, where $M$ is the dimensionless mass flux in the wall jet, $\mathrm{c}$ is the airfoil chord length, $\mathrm{I}_{1}$ is the integral parameter of the $\mathrm{x}$-direction velocity profile, $\mathrm{Z}$ is a parameter in Spalding's velocity profile equation as used by Churchill [1992], and E is an empirical factor for separation/entrainment. The parameter $\mathrm{Z}$ is the ratio of the law-of-the-wall velocity at the outer edge of the boundary layer to the free-stream velocity. From these relationships it is seen that several different locations could be predicted for the location of flow separation, but provide a reasonable approximation for the location of flow separation.

$$
\frac{M\left(\frac{U_{\infty}}{U}\right)^{3} c\left[\frac{\partial C_{p}}{\partial x}\right]_{w}}{I_{1} Z^{2}}<0.126 E^{2}
$$

Newman [1961] experimentally found a limit for the separation location of a wall jet over a cylinder of 240 degrees from the exit of the jet, as shown in Figure 3.7. It was also found during this testing that above a threshold of $4 \times 10^{4}$ the Reynolds number had no further influence on the delay of flow separation. Newman used the relationship 
shown in Equation 3.29 to determine the Reynolds number and the dependence of the variation of the separation location on Re, as shown in Figure 3.7.

$$
\operatorname{Re}=\left[\frac{\left(P-p_{\infty}\right) b a}{\rho v^{2}}\right]^{1 / 2}
$$

Equation 3.29

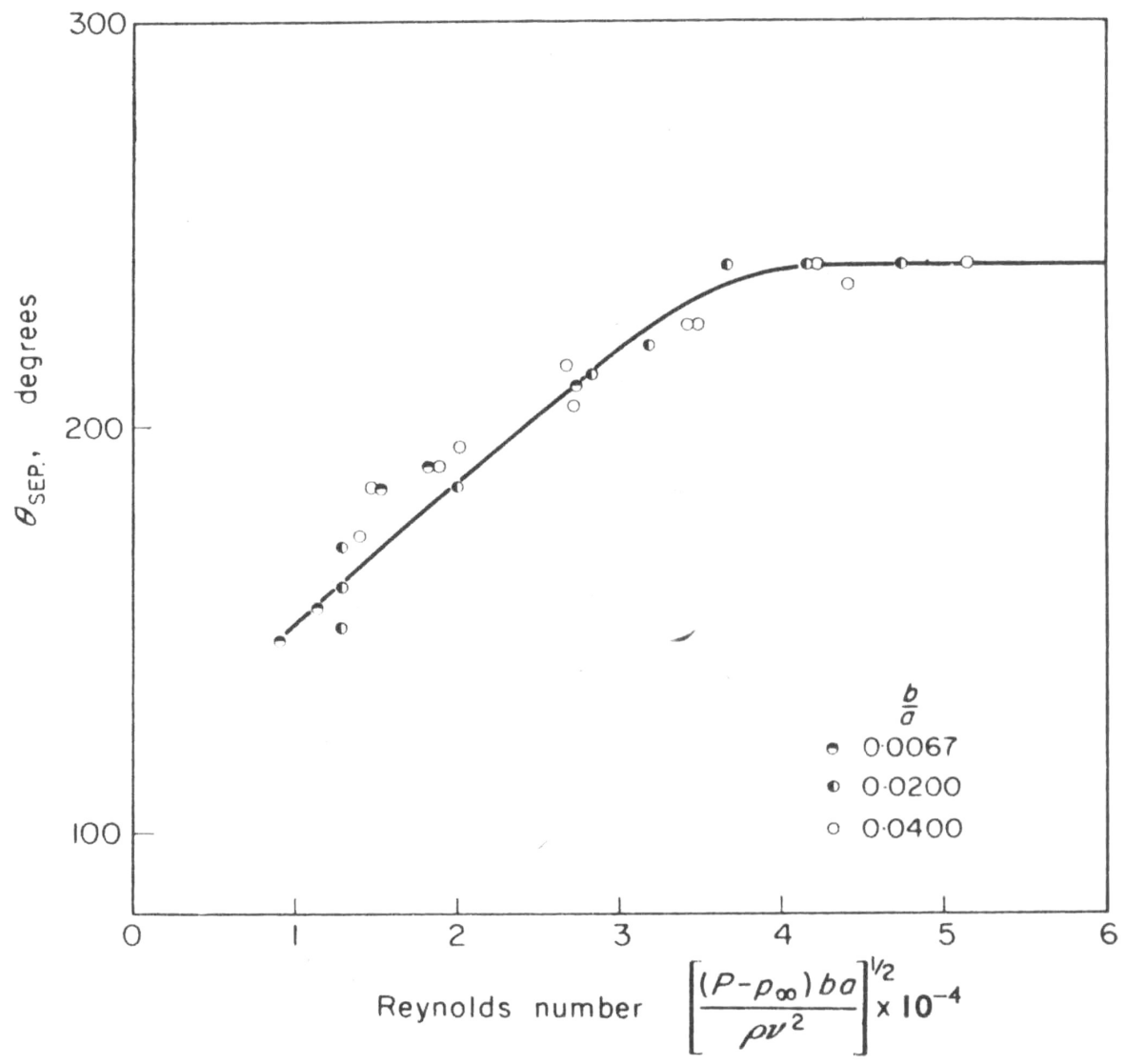

Figure 3.7: The position of separation for small b/a, Newman [1961]

Ambrosiani [1971] states that potential flow methods for calculating the wall jet edge pressure were found to be not applicable for determining pressures in relatively thick boundary layers, due to the inaccuracies found by Kind [1968]. According to Gibbs [1975] the correct blowing slot conditions are those which establish Thwaites condition, while the pressure coefficient of separation is equal between the upper and lower surfaces, which has been experimentally justified by Kind and Maull [1968]. For mild wall pressure gradients with a blowing slot located within the final $5 \%$ of the chord 
length of an airfoil Gibbs [1975] concluded that potential flow calculations were ineffective at estimating the flow.

\subsection{Blowing Coefficient}

The blowing characteristics of the circulation control are described based on the blowing coefficient, $\mathrm{C}_{\mu}$. However, there are multiple definitions of the blowing coefficient where most of them are derived from the relationship shown in Equation 3.30. The blowing coefficient is a function of the density, $\rho$, velocity, $V$, slot thickness, $t$, and the chord length, c, of the jet and free-stream as prescribed by the subscripts $\mathrm{j}$ and $\infty$, respectively. Harness [1970] and Johnson [1975], as well as many others, use this definition of blowing coefficient, but Ambrosiani [1971] developed an alternative definition, shown in Equation 3.31. This relationship describes the blowing coefficient in terms of the ratio of specific heats, $\gamma$, the Mach number of the jet at the slot exit, $\mathrm{M}_{\mathrm{j}}$, slot plenum pressure, $\mathrm{P}_{\mathrm{o}}$. Myer [1972] defined the blowing coefficient in terms of the mass flow per unit span, $\dot{m}$, Equation 3.32 .

$$
\begin{gathered}
C_{\mu}=\frac{\rho_{j} V_{j}^{2} t}{\frac{1}{2} \rho_{\infty} V_{\infty}^{2} c} \\
C_{\mu}=\frac{t}{c} \frac{2 \gamma M_{j}^{2}}{\rho_{\infty} V_{\infty}^{2}} P_{o}\left[1+\frac{\gamma-1}{2} M_{j}^{2}\right]^{\frac{-\gamma}{\gamma-1}}, \\
\text { and } \\
C_{\mu}=\frac{\dot{m} V_{j}}{\frac{1}{2} \rho_{\infty} V_{\infty}^{2} c}
\end{gathered}
$$

Experimental testing conducted by Novak and Cornelius [1986] showed that the slot height is an equally important characteristic. For a constant trailing edge radius, higher lift values were recorded with a smaller slot gap for a given $\mathrm{C}_{\mu}$. The investigators state that this indicates that the overall mixing of the jet with the free stream flow depends on the ratio of velocities. Englar [2005], states a relationship, Equation 3.33, based on isentropic flow from the plenum, to determine the jet velocity based on the 
plenum pressure, $\mathrm{P}_{\mathrm{d}}$, freestream static pressure, $\mathrm{P}_{\infty}$, and the temperature in the plenum duct, $T_{d}$ where $R$ is the gas constant and $\gamma$ is the ratio of specific heats for air, $1716 \mathrm{ft}^{2} /\left(\mathrm{s}^{2}\right.$ $\left.{ }^{\circ} \mathrm{R}\right)$ and 1.4 , respectively.

$$
V_{j}=\sqrt{\frac{2 \gamma R T_{d}}{\gamma-1}\left[1-\left(\frac{P_{\infty}}{P_{d}}\right)^{\frac{\gamma-1}{\gamma}}\right]}
$$

Since the blowing coefficient is dependent on the mass flow rate through the blowing slot, higher $\mathrm{C}_{\mu}$ values require more mass flow. A pulsed blowing slot, or even a cyclic blowing/suction slot can be used to reduce the mass flow requirements. Minimizing the mass flow potentially reduces the sub-system component size and weight, hence making the incorporation of a circulation control system more feasible for aircraft. Englar [2005] states that future investigations of active blowing circulation control should investigate the use of pulsed blowing to reduce the mass flow requirements of the system.

\subsection{Circulation around an Airfoil}

Gibbs [1975] states that the circulation around the airfoil is dependent upon the airfoil shape, free-stream conditions, and blowing rate. Equation 3.34 is the theoretical definition of the circulation defined by the integral around closed path $\mathrm{c}$ of the velocity component, $\bar{V}$, tangent to the surface, $\bar{s}$, as used by Myer [1972].

$$
\Gamma=\oint_{c} \bar{V} \bullet d \bar{s}
$$

Myer [1972] then used the circulation to predict the lift per unit span, L', using the KuttaJoukowski theorem shown in Equation 3.35. However, the use of the Kutta-Joukowski method for alternating airfoils (i.e. helicopter rotors where the pitch constantly changes) has questionable validity. Durand [1976] states that for oscillating airfoils the approximations made within the Kutta-Joukowski theorem are no longer valid and the calculation of lift must be made with more complex means to determine the changes in the flow pattern as the shape oscillates. Durand later discusses methods to determine the forces acting on an airfoil which essentially adds a correction to the Kutta-Joukowski 
method to account for the variation in the flow, as shown in Equation 3.36. In this relationship the subscripts 1 and 2 indicate the lower and upper surface of the airfoil, respectively. Also, $\varphi$ is the potential of the motion around the airfoil and is defined in Equation 3.37, where $\mathrm{w}_{\mathrm{x}}$ is the fluctuation in the $\mathrm{x}$-direction velocity and the index $\mathrm{i}$ indicates that the same relationship holds for both the upper and lower surfaces.

$$
\begin{array}{rlr}
L^{\prime}=\rho_{\infty} V_{\infty} \Gamma, & \text { Equation 3.35 } \\
L^{\prime}=\rho_{\infty} V_{\infty} \Gamma+\rho_{\infty} \frac{\partial}{\partial t}\left(\int\left(\varphi_{2}-\varphi_{1}\right) d x\right), & & \text { Equation 3.36 } \\
\text { and } & \\
\frac{\partial \varphi_{i}}{\partial x}=V+w_{x_{i}} . & \text { Equation 3.37 }
\end{array}
$$

\subsection{Circulation Controlled Airfoils}

Gibbs [1975] also used the Theodorsen method in a potential flow analysis of circulation controlled airfoils. Theodorsen [1931, 1940] transformed the airfoil shape to a circular shape, and then analyzed the potential fluid flow of the circular shape, which favorably agrees with experimental measurements of surface pressure distributions (Gibbs [1975]). The methods derived by Theodorsen are for frictionless, incompressible fluids, thus not truly valid for circulation control airfoils, especially those using active circulation control, due to the compression of air at the blowing slot.

The Coanda simulation conducted by Churchill [1992] under-predicts the decay of the maximum jet velocity, when compared to the experiments conducted by Newman [1961] and Wilson and Goldstein [1976] but the growth of the jet (in thickness) is adequately estimated by the simulation method used. The simulation methods described by Kind [1968], were compared to experimental data and found to be in fair agreement, less that $20 \%$ difference, at low angles of incidence $(0<\alpha<5$ degrees), as seen in Figure 3.8. However, at moderate to large angles of incidence $(5<\alpha<15$ degrees) the agreement digresses, due to the thickening of the upstream boundary layer. Kind [1968] states that the theory becomes inaccurate when the upstream boundary layer thickens enough to 
cause the static pressure coefficient at the edge of the boundary layer to have a value of 1.778 or less.

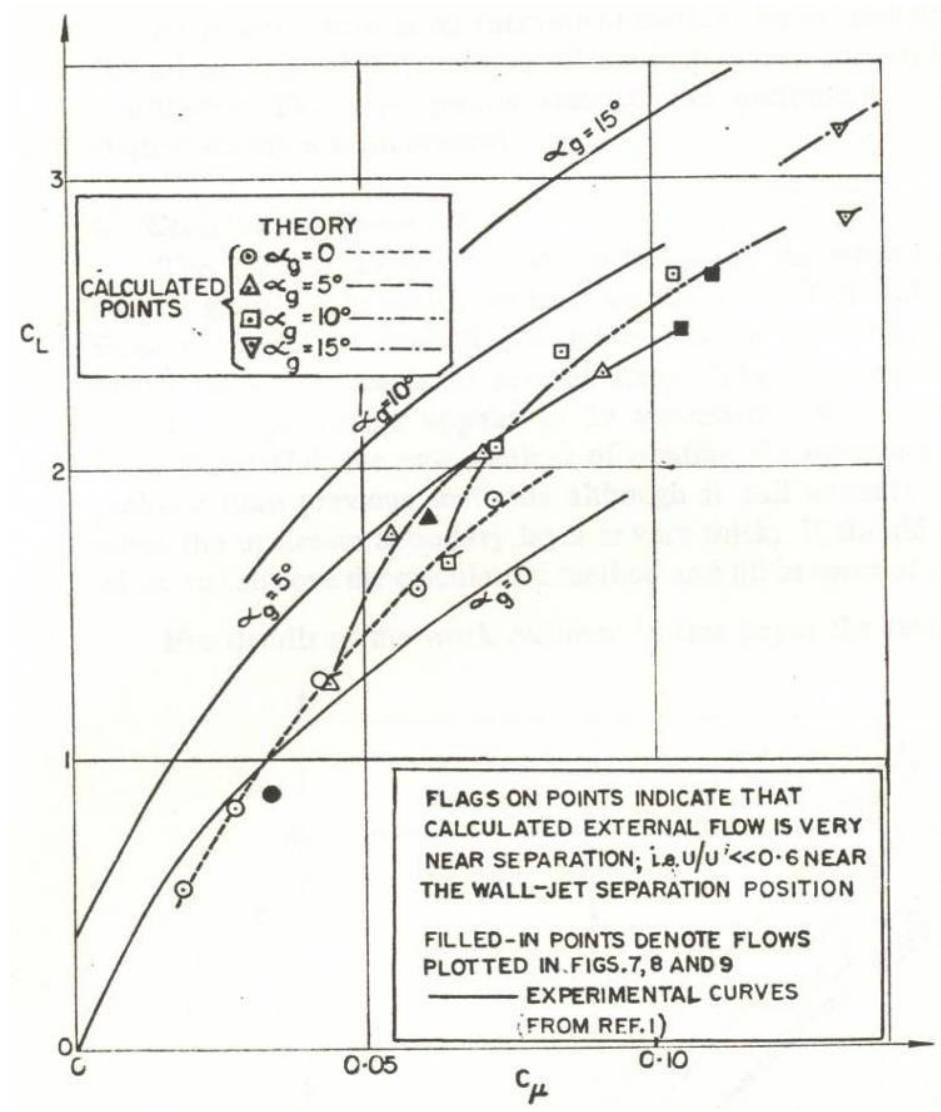

Figure 3.8: Comparison of theory presented in Kind [1968] to experimental curves presented in Kind and Maull [1968]

Another aerodynamic parameter that is of interest is the pitching moment and center of pressure location. From Harness' testing [1970] there was little pitching moment or change in center of pressure location for both of the elliptical airfoil models with different blowing coefficients.

Harness [1970] experimentally found a lift augmentation ratio, the slope of the $C_{1}$ -vs- $\mathrm{C}_{\mu}$ curve, had a maximum value of 25 and 40 for the two elliptical model tests for the cambered ellipse without and with the modified trailing edge. Other experiments, in particular, by Abramson [1975, 2004] have measured lift augmentation ratios greater than 70. Englar et. al. [2001] states that a lift augmentation ratio of 80 is achievable. It has been noted, by Englar [2005] that a two-dimensional lift coefficient as high as 20 can be achieved without moving parts, creating a Super-STOL capability. Johnson [1975] tested 
a straight Coanda jet model as well as a cross-flow jet model which provided for ejection of the jet at angles from $0^{\circ}$ to $90^{\circ}$ relative to the test section flow with jet velocity to freestream velocity ratios of 4.6 and 7.28.

The models tested by Harness [1970] revealed that flow separation over the entire upper surface of the airfoil could be prevented for angles of attack less than 12 degrees. The coefficient of lift is dependent upon the location of rear separation, $\theta$, and effective angle of attack, $\alpha_{e}$, of a 20\%c ellipse can be obtained from Equation 3.38. Harness noted that comparing this relationship to experimental data of the 5\%c cambered airfoil, resulted in a shift in $\theta$ of one degree in the location of the rear stagnation point. This shift in the rear stagnation point was due to the addition of camber, and subsequent increase in lift, which concurs with the work of Myer [1972].

$$
C_{\ell}=\frac{12 \pi}{5} \sin \left(\alpha_{e}+5 \theta\right)
$$

Ambrosiani's [1971] analysis indicated that high lift coefficients could be achieved for relatively low blowing rates, as illustrated in Figure 3.9. At these low blowing rates, the lift coefficient increases with blowing rate but at high blowing rates an asymptotic limit of the lift coefficient is reached. A maximum two-dimensional lift coefficient of 4.58 was found by Myer [1972] for the 20\%c thick, 5\%c cambered airfoil with a blowing coefficient of 0.17 .

The use of pulsed plenum pressures can considerably increase the efficiency of a circulation control system over a constant, high pressure system. Churchill [1992] discusses the alternating growth and decay of the boundary layer, ultimately increasing the entrainment rate from the free stream because of the vortices established at the edge of the boundary layer. The pulsed flow would require less mass flow and thus need less power.

Determination of the drag on a two dimensional airfoil can be estimated by a combination of form drag and viscous drag. Harness [1970] describes form drag as the retarding force experienced by an airfoil due to the resultant pressure forces and the viscous drag is a shear stress component, due to fluid viscosity, in the direction of the free-stream. In using the pressure deficit method to determine the form drag of a 
circulation control airfoil Harness [1970] accounted for the thrust due to the blowing jet by using Equation 3.39. In this relationship the drag coefficient, $\mathrm{C}_{\mathrm{do}}$, was based on the measured drag coefficient, $\Delta \mathrm{C}_{\mathrm{do}}$, and the blowing coefficient, $\mathrm{C}_{\mu}$. The author also noted a large effect on the local dynamic pressure in the flow field downstream of the wing through the introduction of the blowing slot which needs to be considered when using the velocity deficit method to estimate drag. Abramson [2004] developed a relationship for the drag based on the compressor power, $\mathrm{P}_{\text {comp }}$, and intake momentum flux, $\dot{m} V_{\infty}$, as shown in Equation 3.40. Non-dimensionalizing this relationship can be expressed in terms of the blowing coefficient, jet velocity, and freestream velocity, as shown in Equation 3.41.
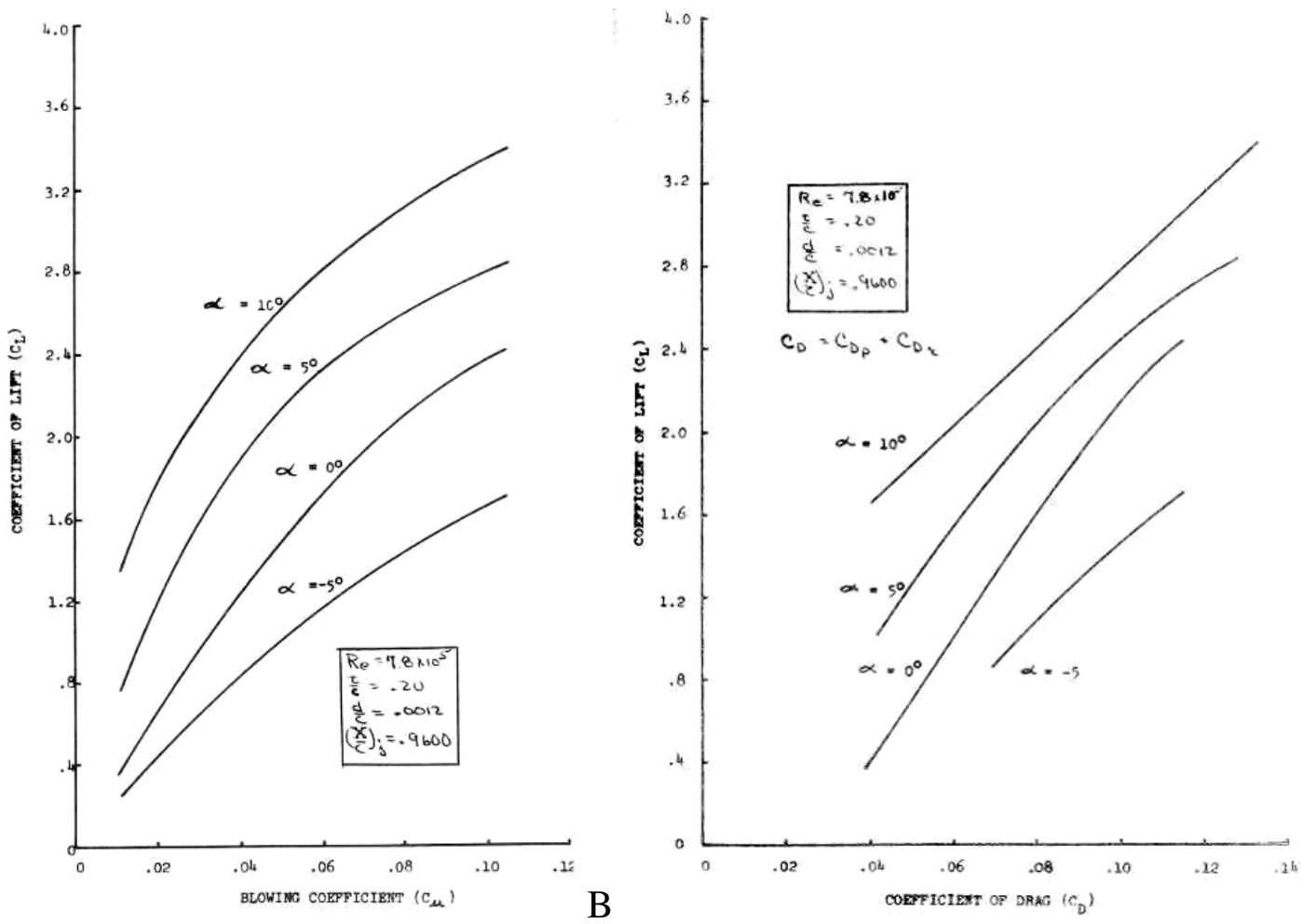

Figure 3.9: Lift (A) and drag (B) coefficients for a circulation controlled elliptical airfoil, Ambrosiani [1971] 


$$
\begin{gathered}
C_{d_{o}}=\underset{\substack{d_{o} \\
\text { and }}}{ }+C_{\mu}, \\
d_{e}=d+\frac{P_{\text {comp }}}{V_{\infty}}+\dot{m} V_{\infty} \\
\text { for } \\
C_{d_{e}}=C_{d}+C_{\mu} \frac{V_{j}}{2 V_{\infty}}+C_{\mu} \frac{V_{\infty}}{V_{j}} .
\end{gathered}
$$

Equation 3.40

Equation 3.41

Englar [2005] lists four additional benefits noticed from prior experimentation, in addition to lift augmentation, for fixed wing aircraft with circulation control force and moment augmentation. These benefits are:

1. only very small movement, or even non-moving, control surfaces required,

2. lift augmentation can be achieved, independent of the airfoil angle of attack,

3. jet turning angle is no longer limited by physical jet exit angle or blown flap deflection angle, and

4. very high force augmentation can be achieved per unit blowing momentum input.

An alternative to using a blowing jet to increase the momentum of the boundary layer fluid is to incorporate a suction slot to remove the low energy boundary layer from the surface of the airfoil. Cerchie et. al. [2006] provide a relationship for the change in lift coefficient, $\Delta \mathrm{C}_{\mathrm{L}}$, based on the volume flow coefficient, $\mathrm{C}_{\mathrm{Q}}$ and the location of the slot when mapped on a "circular path," $\phi$, as shown in Equation 3.42, where the volume flow coefficient is defined by Equation 3.43. The change in drag coefficient due to a suction circulation control device can be found by Equation 3.44. 


$$
\begin{gathered}
\Delta C_{L}=2 C_{Q} \cot \left(\frac{\phi}{2}\right), \\
C_{Q}=\frac{Q}{S U_{\infty}}, \\
\text { and } \\
\Delta C_{D}=2 C_{Q}
\end{gathered}
$$

It was stated by Cerchie et. al. [2006] that there are two major difficulties with a suction type system, the considerable drag increase and the ducting requirements which create operational limits to the application of suction.

\subsection{Trailing Edge Geometry}

The blowing slot not only delays flow separation but it also alters the pressure distribution in the wall jet region, which according to Ambrosiani [1971], could not be theoretically predicted using an ideal-fluid approach. According to Myer [1972] the trailing edge geometry is an important factor in the application of circulation control. Harness [1970] investigated this geometry and found that a round trailing edge yielded the largest influence on the aerodynamic forces. Harness tested two models, the first of which had a 9 inch chord length, a span of $16 \frac{1 / 2}{2}$ inches and was a 20\%c thick elliptical airfoil with 5\%c camber with a blowing slot with thickness t/c of 0.001 and located at an $\mathrm{x} / \mathrm{c}$ of 0.92 . The second model tested by Harness incorporated a modified trailing edge, which shortened the chord-length to $85 / 8$ inches by adding a 5.8\%c radius to the trailing edge. This modification also moved the blowing slot location to an $\mathrm{x} / \mathrm{c}$ of 0.955 .

Two characteristics noticed by Harness [1970], led to the conclusion that the modified trailing edge was more efficient for circulation control. These factors were the presence of relatively high suction peaks at the trailing edge, and the elimination of the slight pressure increase downstream of the slot that was present in the unmodified elliptical airfoil. This second characteristic could also be a factor in the location of the blowing slot. The discrepancies in Harness' results are difficult to separate into the two influences with the models tested. Since only two models were tested by Harness [1970] 
and the difference between them incorporated both a different trailing edge radius and location of the blowing slot $(\mathrm{x} / \mathrm{c})$, which parameter has a greater influence cannot be determined.

Abramson [2004] and Imber et. al. [2005] investigated increasing the range of lift augmentation for a fixed angle of attack through applying circulation control on both the upper and lower surfaces. In this experimental study it was noticed that the introduction of the lower slot geometry did not compromise the lift augmentation of the upper surface blowing. By using the dual blowing slot setup, the range of change in lift was doubled. A trailing edge radius to chord length of 0.067 was tested by Novak and Cornelius [1986], who acknowledge that this ratio is too large for a practical flight system. The increased turning radius allowed for the use of a larger slot height. Wood [1986] noticed that a discontinuity in the radius of curvature leads to a premature jet detachment. Therefore a smooth transition from the nozzle to the Coanda surface is needed.

\subsection{Impact of Sweep Angle on Circulation Control}

Another parameter that can influence the effectiveness of circulation control is the sweep angle. Keener, et. al. [1986] experimentally tested a swept circulation control wing and found that the conventional sweep theory corrections applied to the data adequately described the forces, without additional corrections for changes in blowing efficiency. Conventional sweep theory for a very high aspect ratio wing incorporates trigonometric terms of the sweep angle to determine the flow parameters (speed, pressure) in the normal direction. Equation 3.45 through Equation 3.48 show the relationships for conventional sweep theory for the Mach number, M, dynamic pressure, $\mathrm{q}$, coefficient of pressure, $\mathrm{C}_{\mathrm{p}}$, and coefficient of lift, $\mathrm{C}_{\ell}$, as functions of the local pressure, $\mathrm{P}$, and atmospheric pressure, $\mathrm{P}_{\infty}$, and the sweep angle, $\Lambda$. 


$$
\begin{gathered}
M_{\text {normal }}=M_{\infty} \cos ^{2} \Lambda \\
q_{\text {normal }}=q_{\infty} \cos ^{2} \Lambda \\
C_{p, \text { normal }}=\frac{\left(P-P_{\infty}\right)}{q_{n}} \\
C_{\ell, \text { normal }}=\frac{C_{\ell}}{\cos ^{2} \Lambda}
\end{gathered}
$$

Spaid and Keener [1986] added that the jet flow near the slot is directed normal to the slot. For a wing that is swept towards the aft of the aircraft the jet blows in the inboard direction, toward the fuselage. Additional considerations should be made to account for the primary flow directions of the respective elements (freestream and jet). Likewise, a forward swept wing incorporates an outward component to the flow in the wake of the airfoil.

\subsection{Sources of Error in Circulation Control Investigations}

The complex flow around a circulation control airfoil makes the prediction of the flow difficult. For typical airfoils, with sharp trailing edges, the circulation, $\Gamma$, around the airfoil is determined based on the Kutta condition. However, according to Ambrosiani [1971], for rounded (bluff) trailing edge airfoils the Kutta condition does not apply and the theoretical solution becomes indeterminate. Churchill [1992] stated that the piecewise calculation of the flow field near an airfoil allows for refined calculation of the near airfoil boundary layer without the time penalty of small numerical cells in the entire flow field. The flow field was divided into three components, the potential flow around the wing, the boundary layers of the upper and lower surfaces, and the wall jet, to simplify the calculations of flow parameters. The flow in the high energy jet influences the boundary layer development and separation location, which in turn influences the potential flow in the larger flow field.

Gibbs [1975] discusses yet another difficulty with many circulation controlled airfoils, in that there is a discontinuous radius of curvature, from the truncation of the airfoil at the blowing slot and the rounded trailing edge. During experiments conducted by Kind [1968] and Harness [1970], a feedback in the surface pressure was noticed 
upstream of the wall jet differing from the predicted pressures from a potential flow model. The amount and distance of this feedback depends on the blowing coefficient, with higher blowing coefficients resulting in a larger distance, which was found experimentally to be approximately $10 \%$ of the chord length.

It was noticed that for the experimental model tested by Myer [1972] that a vortex was shed at the airfoil-endplate junction which caused downwash, decreasing the effective angle of attack, relative to the geometric angle of attack. The downwash angle was accounted for to obtain two-dimensional results which can be useful in designing blades and wings. Kind and Maull [1968] state that for models with blowing or suction at small blowing rates $\left(\mathrm{C}_{\operatorname{lmax}} \sim 4\right)$, the interference issues of wind tunnel testing are the same as in the testing of normal (non-circulation controlled) models, provided the chord length of the model is less than one-third of the wind tunnel height.

Another issue of any wind tunnel testing is the interference created by the blockage of the test section. Franke and Harvell [1986] used the standard blockage corrections outlined by Pope in an early edition of Barlow, Rae and Pope [1999]. Equation 3.49 provides a correction for the drag coefficient based on the test section blockage. In these relationships, A is the frontal area of the model exposed to the freestream velocity and $\mathrm{S}$ is the cross-sectional area of the wind tunnel test section.

$$
C_{D}=\frac{C_{D_{\text {meas }}}}{\left(1+\frac{A}{4 S}\right)^{2}}
$$

The effectiveness of circulation control depends on the jet conditions so care must be taken to ensure proper exit conditions. The width of the slot on the model used by Bauman [1976] was found to have a non-uniform slot width, as well as being partially blocked. The inconsistency in the slot width alters the geometry of the wake and the surface pressure distributions around the model, incorporating three-dimensional effects and introducing errors.

The wall interference on the effectiveness of the circulation control jet during experimental testing is also a potential source for error. Owen [2005] estimated the wall boundary layer separation interference/error by correcting the angle of attack by multiplying the lift coefficient by -1.5 and adding to the geometric angle of attack. An 
error greater than $100 \%$ was found to be typical for induced angles of attack less than one degree as noted by Owen [2005].

\subsection{Computational Modeling of Circulation Control}

The computational modeling of the physics occurring within the airflow around a circulation control wing has proven to be difficult, requiring experimental validation of the specific blowing configuration. Shrewsbury [1986] states that the complex flow fields of the circulation control airfoil are governed by highly interactive flow regimes. To adequately model this interactivity analysis, procedures which account for the strongly coupled viscous and inviscid flow regimes are needed. Two deficiencies, as noted by Dvorak and Dash [1986], in computational modeling were the inability to predict the shock structure of under-expanded supersonic jets, and the insensitivities of the calculations to small changes in the Coanda surface geometry. Potential flow based computational techniques can determine the basic "what-if" trends of a proposed circulation control application but have difficulty determining the quantitative "how-to" details of the trailing edge shape, mass flow requirements, and the identity of the performance boundaries of a circulation control system, according to Imber et. al. [2005].

Viegas et. al. [1986] computationally investigated the trailing edge region of a circulation control airfoil using the Reynolds-averaged Navier-Stokes equations. The investigators state that the Navier-Stokes solvers appear to handle the compressibility effects of the transonic, and under-expanded blowing jets. However, significant modification to the current mixing length models are needed in order to handle the multiple streams with different turbulence intensities and length scales at jet and separation points are needed for CFD analysis of circulation control airfoils.

The extreme stream-wise curvature, creating a lift coefficient greater than three, requires the implementation of corrections, according to Viegas et. al. [1986]. The ability to predict the separation point is a critical parameter in determining the performance characteristics of the circulation control airfoil. Most turbulence models have difficulty in this regime thus the selection of turbulence model is important. Linear mathematical models require less computation time but typically have higher errors that exponential relationships for the turbulence, thus a trade-off is made to determine the appropriate 
turbulence model for the geometry. The Cebeci-Smith and Baldwin-Lomax turbulence models were investigated by Viegas et. al. [1986], using a modified boundary layer model. It was concluded from this investigation that higher-order turbulence models should be incorporated in future computational work with circulation control.

A semi-elliptic extension of the parabolic modeling of the airfoil's trailing edge is needed to account for turbulent contributions $\left(\bar{v}^{\prime} \vec{v}^{\prime \prime}\right)$ to the normal pressure variation near strong curvature, according to Dash, et.al. [1986]. A curvature correction for the k- $\varepsilon$ turbulence model, based upon the Richardson number, Ri, and the constants $\mathrm{C}_{1}$ and $\mathrm{C}_{2}$ as defined in Equation 3.50 through Equation 3.52 was used by Dash et. al. [1986].

$$
\begin{aligned}
R i & =-k U\left(\frac{k}{\varepsilon}\right)^{2} \frac{\partial U}{\partial n} \\
C_{1} & =1.43\left(1+C_{c} R i\right) \\
C_{2} & =1.92\left(1-C_{c} R i\right)
\end{aligned}
$$

Equation 3.52

Here, a value of 0.2 for $\mathrm{C}_{\mathrm{c}}$ yielded optimal predictions for curved boundary layer flows. Shrewsbury [1986] successfully used Navier-Stokes methods to computationally solve the aerodynamics around circulation control airfoils.

An offset in the angle of attack was noticed by Shrewsbury [1986] for the computational lift coefficient, at -2 degrees angle of attack for the computational model corresponded to the zero degree incidence angle experimental value of 4.55 found by Novak and Cornellius [1986]. An alternative correction for the computational angle of attack, shown below in Equation 3.53, was used by Pulliam et. al. [1986] to correlate the computational data to experimentally measured data.

$$
\Delta \alpha_{\text {deg }}=-1.5 C_{\ell_{\text {exp }}}
$$


Owen [2005] states that more research and analysis is required in order to properly establish a reliable data base for CFD code validation.

Paterson and Baker [2006] were able to get a Reynolds Averaged Numerical Solver (RANS) model to successfully predict the pressure distribution trends with a blended k- $\omega$ and k- $\varepsilon$ turbulence model for the NCCR airfoil with large jet-momentum coefficients. Fluent's steady-state, two-dimensional Navier Stokes segregated solver was used by Chang et. al. [2006] to model the flow around a circulation control airfoil. The authors noted that for a circular-arc trailing edge, the full Reynolds Stress turbulence model fairly accurately predicted the Coanda jet detachment point. For most cases the lift coefficient was found to be approximately 0.5 lower than the comparable experimental values, but the trends in lift augmentation were correctly predicted for a range of angles of attack, jet slot heights, and blowing coefficients.

\subsection{Rotorcraft Applications}

The asymmetry of lift which is generated by a disparity in velocities experienced by advancing and retreating blades in maneuvering flight, is traditionally overcome by altering the blades angle of attack to maintain a symmetric lift profile throughout the rotor disc. Bauman [1976] and Reader and Wilkerson [1977] introduce the concept of using properly scheduled blowing of circulation control slots instead of the pitching of the blade. This in turn can lead to the removal of the swash-plate and associated linkages by designing a pneumatic valving system to respond to the traditional cyclic and collective inputs from a pilot. Another benefit of removing the swash-plate is a simplified external shape, presenting a cleaner profile for total aircraft drag considerations.

Bauman [1976] states that by controlling the blowing velocity it is possible to control the lift generated by the airfoil without changing the angle of attack. In this experimental setup the plenum pressures were controlled by a rotating valve, made of an elliptical cam and two globe valves, and used to generate the sinusoidal blowing coefficient of the form expressed in Equation 3.54, where the constants A and B are adjusted by the globe valves with a frequency of oscillation, $\omega$, and time, $t$. 


$$
C_{\mu}=A+B \sin (\omega t)
$$

The helicopter specific testing conducted by Bauman [1976] had limited scope since during this research effort, only a -5 degree angle of attack, which is the zero-lift angle of attack, was investigated. The airfoil shape was a $20 \%$ c thick elliptical airfoil with $3.3 \% \mathrm{c}$ camber and had a 0.010 inch blowing slot located at $0.95 \mathrm{c}$. Another notable characteristic of the experimental setup used was that the valve controlling the oscillations was outside of the wind tunnel in the conduit to the model.

A more refined cam-controlled valve was used by Reader [1976] to evaluate the effect of reduced cam size, and the dynamic effects of a collector ring on the airflow from the hub to the ducts in the blade. This model's hub valving system provided data for evaluating stratification (defined by Reader as the velocity difference between portions of the plenum inlet), pressure recovery in the blades, and the shape of the pressure wave in the blade.

By controlling the blowing velocity both cyclic and collective controls can be achieved on a fixed root rotor with circulation control. Reader and Wilkerson [1977] describe the concept of a circulation control rotor has having the potential to reduce the rotor speed (rpm) in a thrust compounded helicopter with speeds approaching 400 knots. The authors also introduce the concept of using circulation control on a stoppable rotor on a craft that could reach speeds approaching Mach 1.0.

Kumagai [1984] performed a numerical analysis on a once per revolution cyclic tip section which created a cyclic moment to longitudinally trim the rotor. Several assumptions were made by the author during this analysis. The first assumption was that the induced velocity was modeled in terms of a prescribed wake by defining the inflow angle distributions. The wake profile was a combination of uniform and non-uniform downwash and accounted for the wake induced velocity and the movement of the blade. The author also based the aerodynamic parameters on the two-dimensional blade element theory. The lift, drag, and moment coefficients were approximated using the PrantlGlauert similarity to compensate for compressibility. The aerodynamic damping was estimated by the unsteady quasi vortex lattice method and was the only unsteady effect that was analyzed. The final assumption made during this analysis was that the center of 
gravity of the free-tip section was designed to coincide with the pitch axis to minimize the moment of inertia. The free-tip was shown to allow the inboard section of the blade to have a larger twist, resulting in a better overall performance.

A circulation control flap response of approximately two per revolution was used by Chopra and Hong [1986], and the amplitude of the response increased with the advance ratio, $\mu$. Numerical results were determined by the authors for a 4-bladed circulation control rotor where the blade flap frequency was 2.3 per rev., blade lagfrequency was 2.6 cycles per rev. and the torsion rotating frequency of 18 cycles per rev., assuming no structural damping in the blades. Chopra and Hong state that for high forward flight speeds $(\mu>0.4)$ the lag response becomes unstable at low thrust levels.

Englar [2005] summarized the performance outcomes of the 1979 test flights of the Kaman H-2 helicopter, Circulation Control Rotor Flight Demonstrator. One difficulty discussed was the control system response phasing problems which limited the flight test envelope of the aircraft. The test flights demonstrated the ability to substitute the pneumatics for mechanical blade lift and control devices for hover and limited forward flight. The possibility of using circulation control in a higher harmonic (> one per rev.) control of helicopter components can possibly be used to eliminate some rotor-induced vibrations. Despite these potential benefits, the considerable limitations to the forward flight speed limited further research into their circulation control rotor.

In testing conducted by Alexander et. al. [2006] it was noted for the relatively low freestream velocity, $M=0.3$, the effectiveness of the circulation control increased with decreasing slot height and decreasing Coanda surface elliptical ratio. Testing at a Mach number of 0.8 yielded that the effectiveness increased with decreasing slot height and increasing Coanda surface elliptical ratio. Thus, for a helicopter rotor the slot height and trailing edge shape could vary with respect to the span of the rotor blade.

\subsubsection{Reverse Blowing Circulation Control Rotor}

An aircraft making use of the rotor system of Reader and Wilkerson [1977] is compressor power and/or trim limited in forward speed. A throttling mechanism in the rotor head provided control over the cyclic and collective rotor control requirements. Bauman [1976] investigated use of a sinusoidal input whose frequency was equal to the 
rotational frequency of the main rotor. In this investigation a system was designed and fabricated to generate a sinusoidal blowing coefficient by controlling the plenum pressure, to determine the response of lift coefficient to the oscillating circulation control. The peak surface pressure oscillation was found to lag behind the peak plenum pressure by approximately 50 to 70 degrees in azimuth.

By using an airfoil with the same leading and trailing edge profiles, Reader and Wilkerson [1976, 1977] introduced the concept of a Reverse Blowing - Circulation Control Rotor (RB-CCR). A four bladed, 80-inch diameter RB-CCR model was tested in the 8x10 Foot North Subsonic Wind Tunnel of the Aviation and Surface Effect Department of the David W. Taylor Naval Ship Research and Development Center. The rotor had a varying airfoil profile; Figure 3.10 shows the root and tip profiles. The root airfoil was a $20 \%$ c thick ellipse with a $5 \%$ c camber, and the tip profile was a $15 \%$ c thick ellipse with no camber. Blowing slots were placed at $3.2 \% \mathrm{c}$ and $96.8 \% \mathrm{c}$ with a slot thickness of $0.2 \%$, with a constant chord length of 5.0 inches, throughout the blade. The testing of this rotor verified the capability for efficient operation from hover to an advance ratio, $\mu$, of up to 4.0. The addition of the leading edge slot allowed for the inversion of blowing on the retreating blade when the flight speed is greater than the blades rotational speed, mitigating retreating blade stall. At high speed the alternating blowing scheme was used with the blades trailing edge slot on for azimuth positions from 0 to 180 degrees and the leading edge slot operating for azimuth positions from 180 to 360 degrees. 


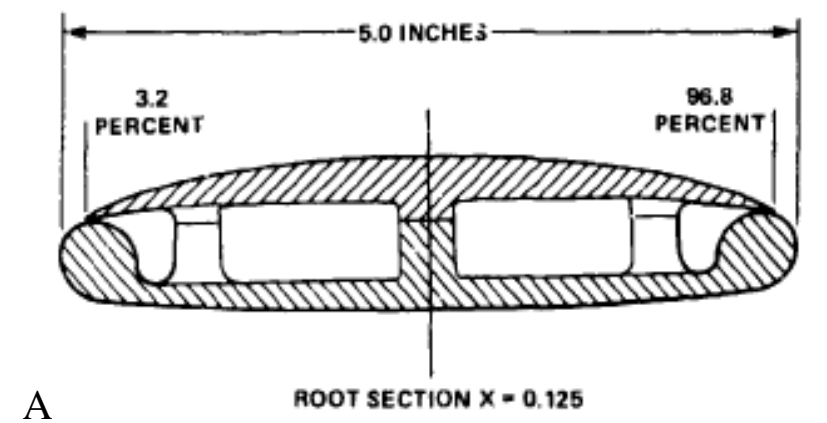

A

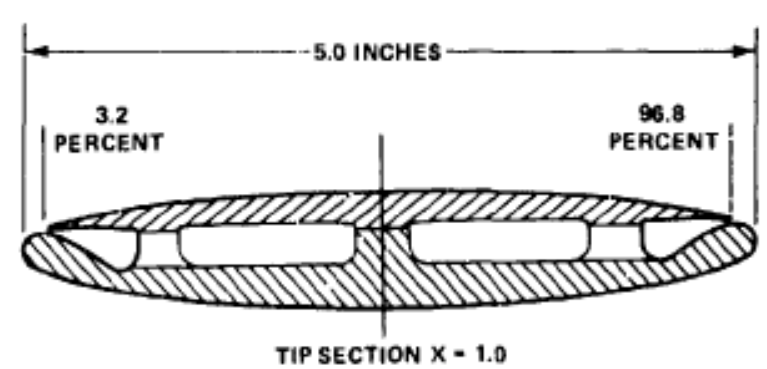

B

Figure 3.10: Root (A) and tip (B) airfoil profile shapes used in the reverse blowing circulation control rotor, Reader and Wilkerson [1977]

The strategy for the use of circulation control was determined based on the advance ratio in which the aircraft is operating. The operational flight envelope was divided into three parts by Reader and Wilkerson [1976, 1977]: conventional operation $(\mu<0.5)$, transitional operation $(0.5 \leq \mu \leq 1.4)$, and cruise operation $(\mu>1.4)$. In the conventional flight regime only the trailing edge blowing is used with a once per revolution cyclic control. At transitional advance ratios a dual blowing scheme is employed on the retreating blade and a two pulse per revolution cosine control signal is added to increase the lift on the fore and aft positions of the rotor disc. As the Mach number at the tip of the advancing blade approaches 0.9 the rotor $\mathrm{rpm}$ is reduced to prevent drag divergence to allow for higher flight speeds.

The blowing rates for the RB-CCR of Reader [1976] were controlled at the hub of the rotor. A two cam system would provide airflow to the blowing slots for the once per and two per revolution schemes. The collective lift coefficient was controlled by a collector ring that altered the area which was not covered by the cams, as illustrated in Figure 3.11. 


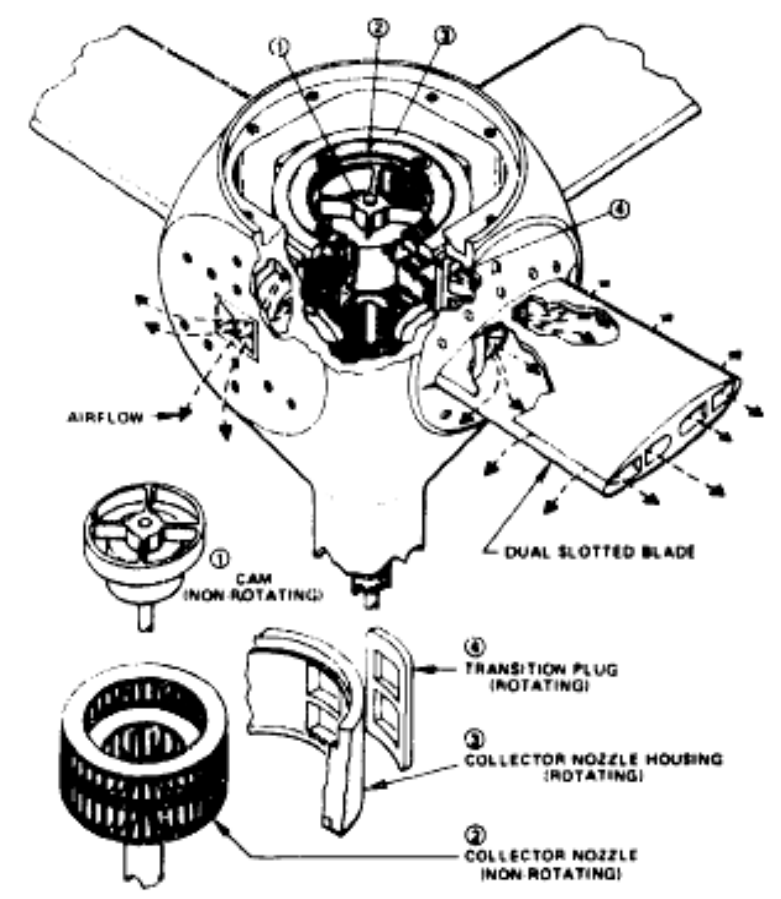

Figure 3.11: Reverse blowing circulation control rotor hub, Reader and Wilkerson [1977]

Five criteria were used by Reader [1976] to evaluate the hub and rotor performance. The first was the minimal presence of stratification which is caused by the incomplete mixing of the collective and cyclic components of the airflow. Stratification of the air flow caused the leading and trailing edge pressure plenums to have unequal pressures. The ability to vary the area ratio (collector open area to the slot area) with azimuth position was the second evaluation criteria. A third criterion was the ability to maintain a proper sinusoidal wave blowing rate shape throughout the possible flight regimes. Maintaining a maximum pressure ratio (pressure in the blade to the pressure at the hub) greater than 0.75 and ease of sealing the system are the final two evaluation criteria. Reader and Wilkerson [1977] state that the RB-CCR model demonstrated both the lift and trim capability needed to reduce the rotor speed and be able to operate at a high advance ratio for cruise.

A further investigation, conducted by McLachlan [1986], found that during simultaneous leading and trailing edge blowing, the leading edge blowing reduces the maximum value of lift, and increases the unsteadiness of the lift. An alternative configuration is discussed by Imber et. al. [2005], a self driven rotary thruster which 
combines the Coanda type circulation control aerodynamics with the cold cycle reaction drive technology.

\subsubsection{Rotor Blade Lift Distribution}

Meyer and Falabella [1953] conducted tests on a two bladed model rotor with a NACA 0015 airfoil with a 3 inch chord length and a rotor diameter of 5 feet to measure the aerodynamic loading on the rotor. Figure 3.12 shows the loading profile determined by measuring the surface pressure profiles at several span-wise locations with a pitch angle of 8 degrees with no twist. The increased velocity as the span-wise location is increased is a major contributing factor to the majority of the lift being on the outboard portion of the blade. The total lift generated in hover by a rotor blade is based on the integral of the curve over the radius of the rotor. Accounting for maneuvering flight is typically done by integrating the pressure contour of the rotor disc.

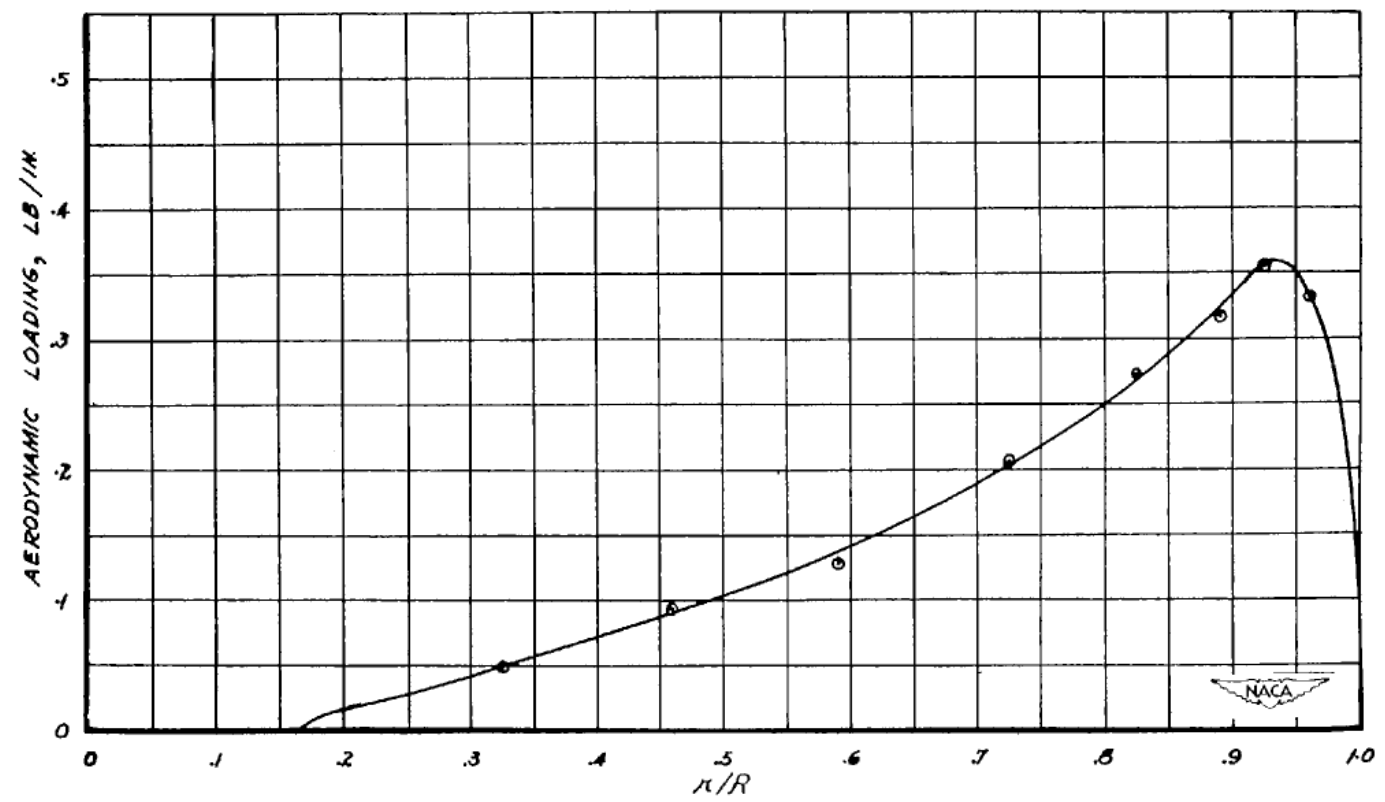

Figure 3.12: Spanwise loading of a model rotor blade, Meyer and Falabella [1953]

\subsubsection{Flow Development Response Time}

Smith, et al. [2002] introduced the use of a blowing slot controlled by piezoelectric materials at the surface of the slot. This would allow for the control of the 
blowing slot at the surface of the blade, minimizing the time delay in the system. Reducing the response time of the system has significant potential to overcome the difficulties experienced by Bauman [1976], Reader [1976], and Reader and Wilkerson [1977] with the frequency of the blowing system when it is controlled at the hub.

Friedman, Arieli and Levy (2007) conducted a computational investigation into determining the time required for the force response to a change in exit velocity of a circulation control blowing slot. In their analysis a time required was approximately 0.070 seconds with the use of four control points per rotor revolution. Though this is slightly higher than the 0.050 second time desired at a rotor speed of $300 \mathrm{rpm}$, slowing the rotor to $200 \mathrm{rpm}$ would increase the required response time to 0.075 seconds, within this predicted response time.

Combining the estimated times, to fill the plenum and develop new flow characteristics, the total response time of a 10 foot long circulation controlled blade with the valve at the root would be approximately 0.156 seconds (96 RPM with 4 control points per revolution). Utilizing the rotating trailing edge valve the time to establish the new steady-state lift value would be 0.070 seconds (214 RPM with 4 control points per revolution). Both of these times ( 0.156 seconds and 0.070 seconds) do not account for the time required to open the valve, an addition of approximately 0.030 seconds, which could be accounted for by timing the slot to account for this 0.030 seconds. Otherwise, the total estimated time required to open the valve and establish the new force magnitude would be 0.100 seconds which corresponds to 150 RPM with the four control points per revolution.

Another important aspect of a circulation control system is the mass flow and compressor power requirements of the system itself. This is typically described by a mass or volumetric flow rate of air required to obtain the resulting increase in lift. The configuration analyzed by Ambrosiani [1971] required a plenum pressure of 2448 pounds per square foot (17 psi) to get a jet Mach number of 0.49 and a blowing coefficient of 0.0706 . This blowing coefficient corresponded to a lift coefficient of approximately 2.0 for the angle of attack of 0 degrees and approximately 3.0 for a 10 degree angle of attack, a $525 \%$ and $220 \%$ improvement, respectively. 


\subsection{Summary}

The literature reviewed herein was focused on the application of circulation control to an aerodynamic shape, namely an airfoil. Also provided were specific applications to rotorcraft main rotor blades. The downfalls experienced during these early helicopter applications were primarily related to the response times of the system, which can be overcome by relocating the valves from the hub of the rotor to the surface of the blade.

Both experimental and numerical studies were reviewed that provided insight into the difficulties associated with modeling circulation control airfoils. The differences between the numerical predictions and the experimental results have led to the desire to conduct an experimental study into the effectiveness of surface located valves and serve as a verification case for the computational analysis. 


\section{Preliminary Calculations}

Initial estimates of key elements were calculated to determine reasonable guidelines for parameters such as the response time of the freestream, and the plenum filling time required for a 10 foot radius rotor blade. In addition to these calculations a comparable airfoil was selected to verify that the values of force coefficients are within reasonable bounds.

\subsection{Required Response Time}

The application of circulation control to the main rotor of a helicopter requires a systematic response that is coordinated with the pilot controls to simulate the collective and cyclic control of conventional rotorcraft. There are effectively two response times of concern for the current circulation control model: the time to open the rotary valve and the time to develop the change in flow streamlines, and hence, to also change the magnitude of lift. The present experimental testing will incorporate a relatively high sampling rate $(20 \mathrm{kHz})$ for data to accurately determine the settling time of the force upon the opening of the rotary valve. The time to open the valve will be determined based on the opening time of the solenoid used to open the valve.

The primary issue to the application of circulation control to a helicopter main rotor is the limitation placed upon the performance of the aircraft by the response time of the blowing to establish the new lift force magnitude. This limitation is primarily due to the reaction time of the circulation control system. The previous applications of circulation control to rotorcraft have utilized a cam system located within the hub of the rotor to actuate or control the blowing rate. The time lag of this system considerably hindered the performance of the aircraft due to the need to fill the plenum inside the rotor blade, in addition to the times required to open the valve and establish the new flow streamlines around the blade.

Using the typical rotor speeds of 200-400 rpm and assuming that four control points in the blades' path would be necessary per rotor revolution, a required response 
time of the system would be approximately $55 \mathrm{msec}$, as seen in Figure 4.1. For this preliminary time estimate four control points were specified per revolution for the blade.

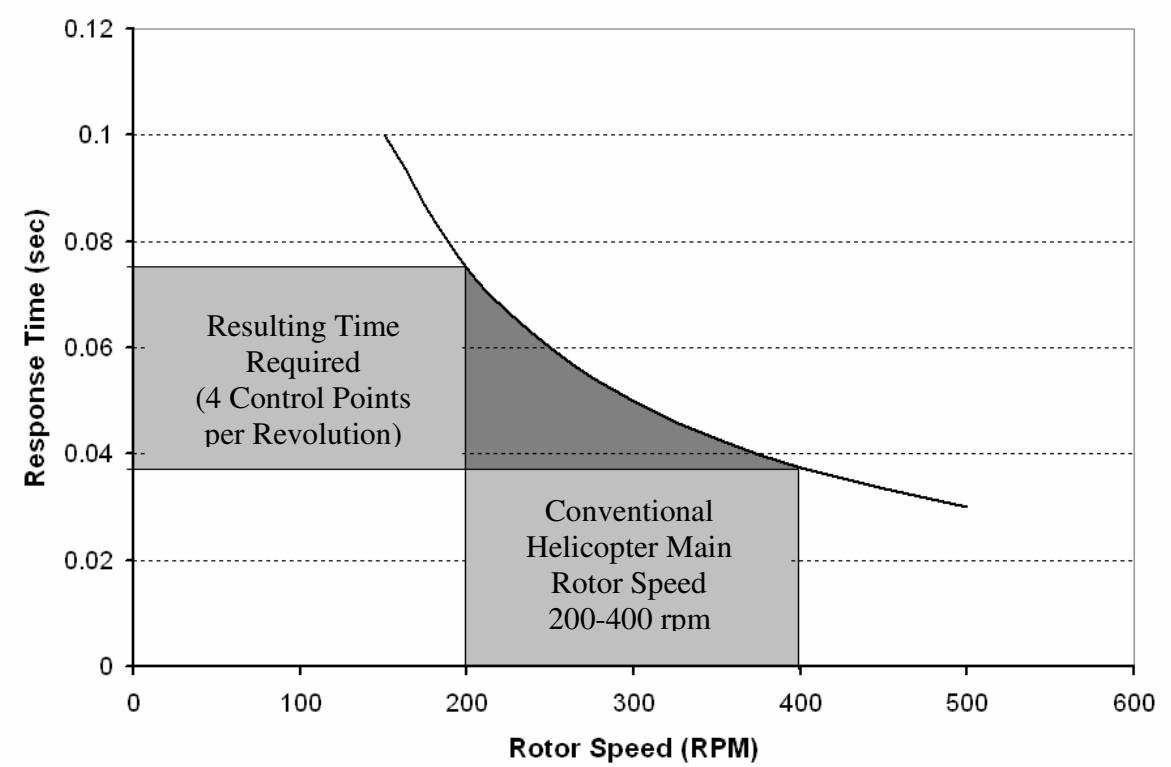

Figure 4.1: Response time required based on typical rotor speeds

\subsection{Blade Filling Time Estimation}

Based on the dimensions used in the analysis by Bauman [1976] the time to fill the blades plenum was estimated for the 10 foot span rotor blade (20 foot diameter rotor) with a supply pressure of $15 \mathrm{psig}$, where it was determined that 0.042 seconds would be required to pressurize the blade with a plenum volume of $0.242 \mathrm{ft}^{3}$. However, this 0.042 second time estimate would be to fill the plenum provided there are no exits, i.e. no open blowing slots.

The centrifugal acceleration and corresponding forces were calculated using Equation 4.1 based on the radius, r, and rotational speed, $\omega$. Given the mass of an air molecule is $6.4 \times 10^{-26} \mathrm{lbm}$, the forces generated by the rotational motion, as shown in Table 4.1, were neglected in this preliminary analysis. The pressure force is $2.9 \times 10^{-15}$ lbf, orders of magnitude greater than the centrifugal force.

$$
a_{r}=-r \omega^{2}
$$


Table 4.1: Calculated centrifugal acceleration and corresponding force on an air molecule for a rotor speed of $350 \mathrm{rpm}$

\begin{tabular}{|c|c|c|}
\hline Radius & Centrifugal Acceleration & Force on Air Molecule \\
\hline $\mathrm{ft}$ & $\mathrm{ft} / \mathrm{s}^{\wedge} 2$ & $\mathrm{lbf}$ \\
\hline 1 & -1340 & $-2.66 \mathrm{E}-24$ \\
\hline 2 & -2690 & $-5.33 \mathrm{E}-24$ \\
\hline 3 & -4030 & $-7.99 \mathrm{E}-24$ \\
\hline 4 & -5370 & $-1.07 \mathrm{E}-23$ \\
\hline 5 & -6720 & $-1.33 \mathrm{E}-23$ \\
\hline 6 & -8060 & $-1.60 \mathrm{E}-23$ \\
\hline 7 & -9400 & $-1.87 \mathrm{E}-23$ \\
\hline 8 & -10700 & $-2.13 \mathrm{E}-23$ \\
\hline 9 & -12100 & $-2.40 \mathrm{E}-23$ \\
\hline 10 & -13400 & $-2.66 \mathrm{E}-23$ \\
\hline 11 & -14800 & $-2.93 \mathrm{E}-23$ \\
\hline 12 & -16100 & $-3.20 \mathrm{E}-23$ \\
\hline 13 & -17500 & $-3.46 \mathrm{E}-23$ \\
\hline 14 & -18800 & $-3.73 \mathrm{E}-23$ \\
\hline 15 & -20100 & $-4.00 \mathrm{E}-23$ \\
\hline
\end{tabular}

This analysis was then expanded to include constantly open blowing slots with a height of 0.007 inches and span 60 percent (72 inches, or 6 feet) of the 10 foot blade, resulting in an exit area of $0.5 \mathrm{in}^{2}$. Using the isothermal density and pressure relationship, at $70^{\circ} \mathrm{F}$ and an atmospheric pressure of 14.696 psia, shown in Figure 4.2 from the Engineering Toolbox [2007] the time required to fill the plenum was determined to be $86 \mathrm{msec}$.

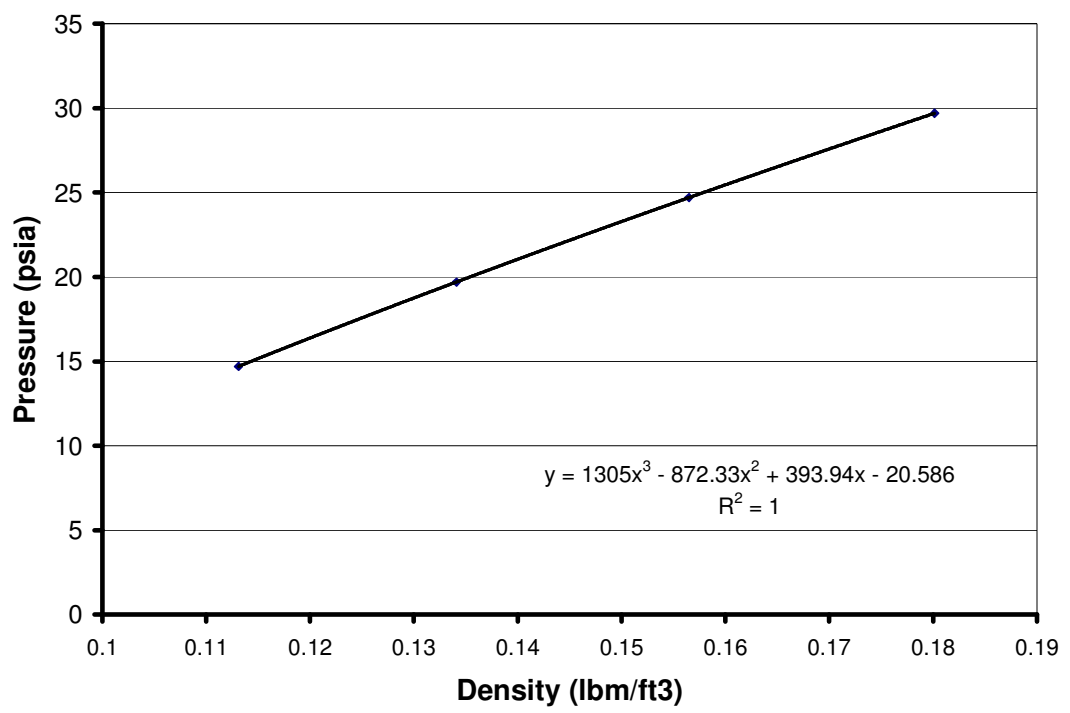

Figure 4.2: Relationship between density and pressure for compressed air [The Engineering Toolbox, 2007]. 
The 0.086 seconds filling time estimate was found assuming an inlet pressure of $15 \mathrm{psig}$, resulting in a plenum pressure of $10.8 \mathrm{psig}$ and steady slot velocity of $1010 \mathrm{ft} / \mathrm{s}$, as seen in Figure 4.3 and Figure 4.4, respectively. It was noticed that the plenum pressure is considerably less than the 15 psig supplied pressure when the exit is added to the system. It should also be noticed that this analysis does not account for any spanwise variation in the parameters in the plenum which would be anticipated. A further, more detailed analysis or an experimental study would be required to obtain a better understanding of the spanwise distribution of properties (i.e. exit velocity) with the threedimensional aspects of the blown air supply plenum(s).

An iterative approach was used to calculate the plenum pressure and the velocities (inlet and exit) from the blades' plenum based on an accumulated mass analysis. The 0.086 seconds calculated in this analysis is only the time required to build up a steady pressure inside the blade. The time required for the freesteam to interact with the circulation control jet and develop the augmented forces still needs to be accounted for, thus restricting the rotor speed to $150 \mathrm{rpm}$ or less according to the analysis in the Section 4.1 of this thesis.

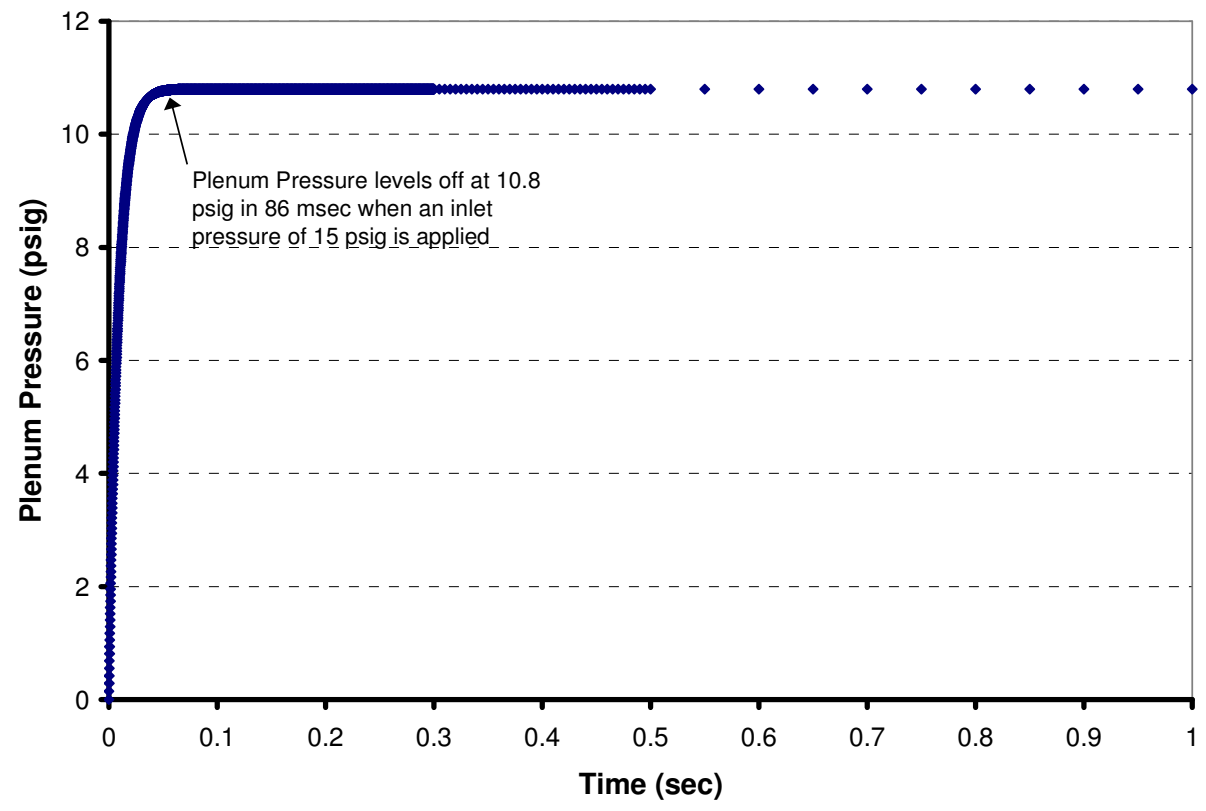

Figure 4.3: Plenum pressure of the system as dependent upon time 


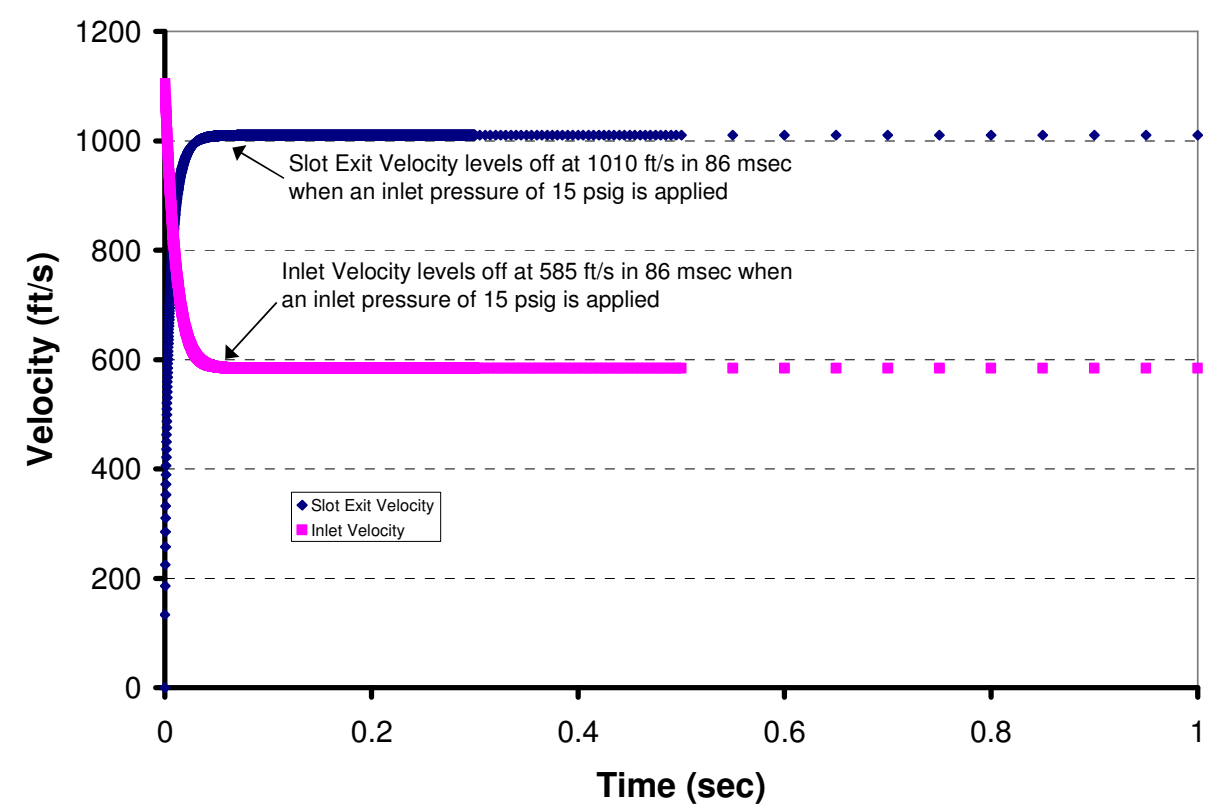

Figure 4.4: Exit and inlet velocities of the system as dependent upon time

\subsection{Comparable Airfoil NACA 0010-34}

For comparison purposes an airfoil, the NACA 0010-34, was selected due to the leading edge similarities to the elliptic profile of the current CCHB model. The lift curve and drag polar for the NACA 0010-34 airfoil was found in Abbott and vonDoenhoff [1959], and is shown in Table 4.2 and Table 4.3. There are still noticeable differences between the NACA airfoil and the CCHB airfoil, due to the rounded trailing edge shape as shown in Figure 4.5.

Table 4.2: Lift coefficient for the NACA 0010-34 airfoil, Abbott and vonDoenhoff [1959]

\begin{tabular}{|c|c|}
\hline$A \circ A$ (deg) & $\mathrm{C}_{\mathrm{l}}$ \\
\hline-2 & -0.2 \\
\hline 0 & 0 \\
\hline 2 & 0.2 \\
\hline 4 & 0.4 \\
\hline 6 & 0.6 \\
\hline 8 & 0.75 \\
\hline 10 & 0.8 \\
\hline 12 & 0.75 \\
\hline 14 & 0.725 \\
\hline
\end{tabular}


Table 4.3: Drag coefficient for the NACA 0010-34 airfoil, Abbott and vonDoenhoff [1959]

\begin{tabular}{|c|c|}
\hline$C_{l}$ & $C_{d}$ \\
\hline-0.7 & 0.0105 \\
\hline-0.6 & 0.009 \\
\hline-0.5 & 0.008 \\
\hline-0.4 & 0.0075 \\
\hline-0.3 & 0.006 \\
\hline-0.2 & 0.0045 \\
\hline-0.1 & 0.0045 \\
\hline 0 & 0.0045 \\
\hline 0.1 & 0.0045 \\
\hline 0.2 & 0.0045 \\
\hline 0.3 & 0.005 \\
\hline 0.4 & 0.007 \\
\hline 0.5 & 0.0085 \\
\hline 0.6 & 0.0095 \\
\hline
\end{tabular}

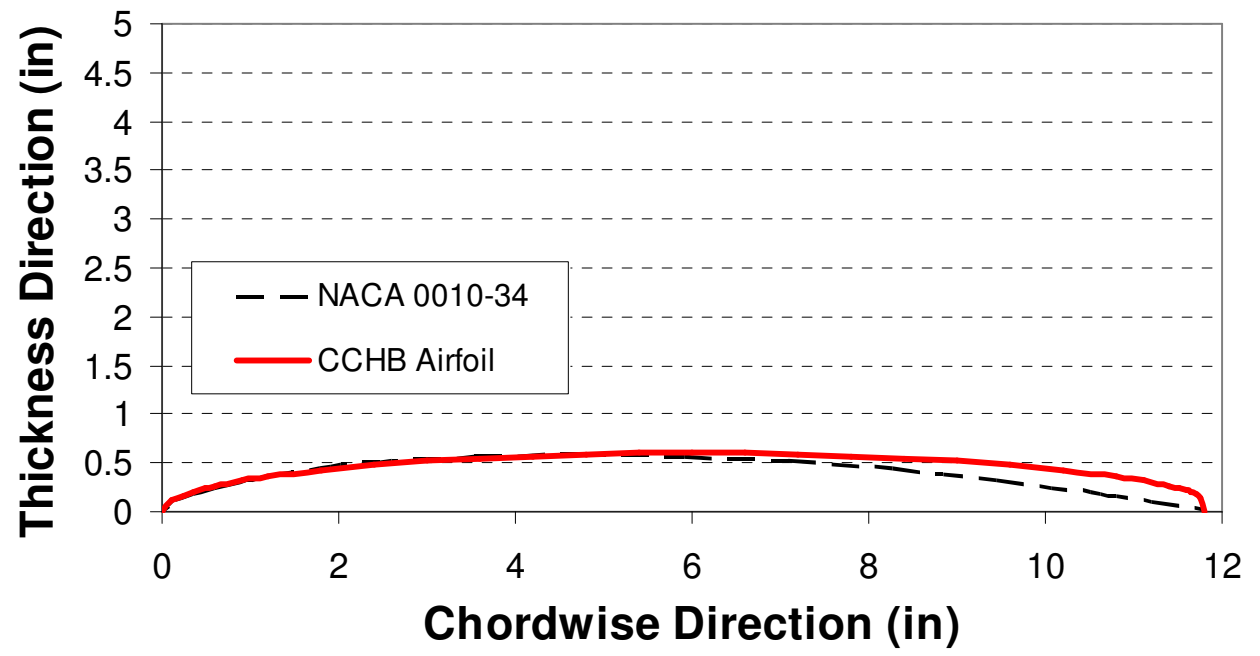

Figure 4.5: Profile comparison of the CCHB Airfoil to the NACA 0010-34

\subsection{Test Section Blockage Considerations}

The test section blockage ratio, BR, as defined in Equation 4.2, was used to determine an appropriate chord length of the model.

$$
B R[\%]=100 \frac{A_{\text {model }}}{A_{\text {tunnel }}}
$$

Equation 4.2 
This relationship was used for the wind tunnel test section, cross sectional area, $\mathrm{A}_{\text {tunnel }}$, to non-dimensionalize the frontal area of the model, $\mathrm{A}_{\text {model }}$. The frontal area of the model was determined based on the chordlength, c, thickness of the airfoil, $\mathrm{t}$, span of the model, $\mathrm{b}$, and the angle of attack, a, as defined in Equation 4.3.

$$
A_{\text {model }}=(c \sin (\alpha)+t \cos (\alpha)) b
$$

Three primary airfoil shapes were considered for this preliminary model, the NACA 0009, NACA 0012 and a 10:1 ellipse. Figure 4.6 shows the blockage ratio determined for the selected airfoils with different chordlengths; Appendix A summarizes the data used to create this figure. The blockage ratio was also determined for various chordlengths of the three airfoil shapes as summarized in Figure 4.7.

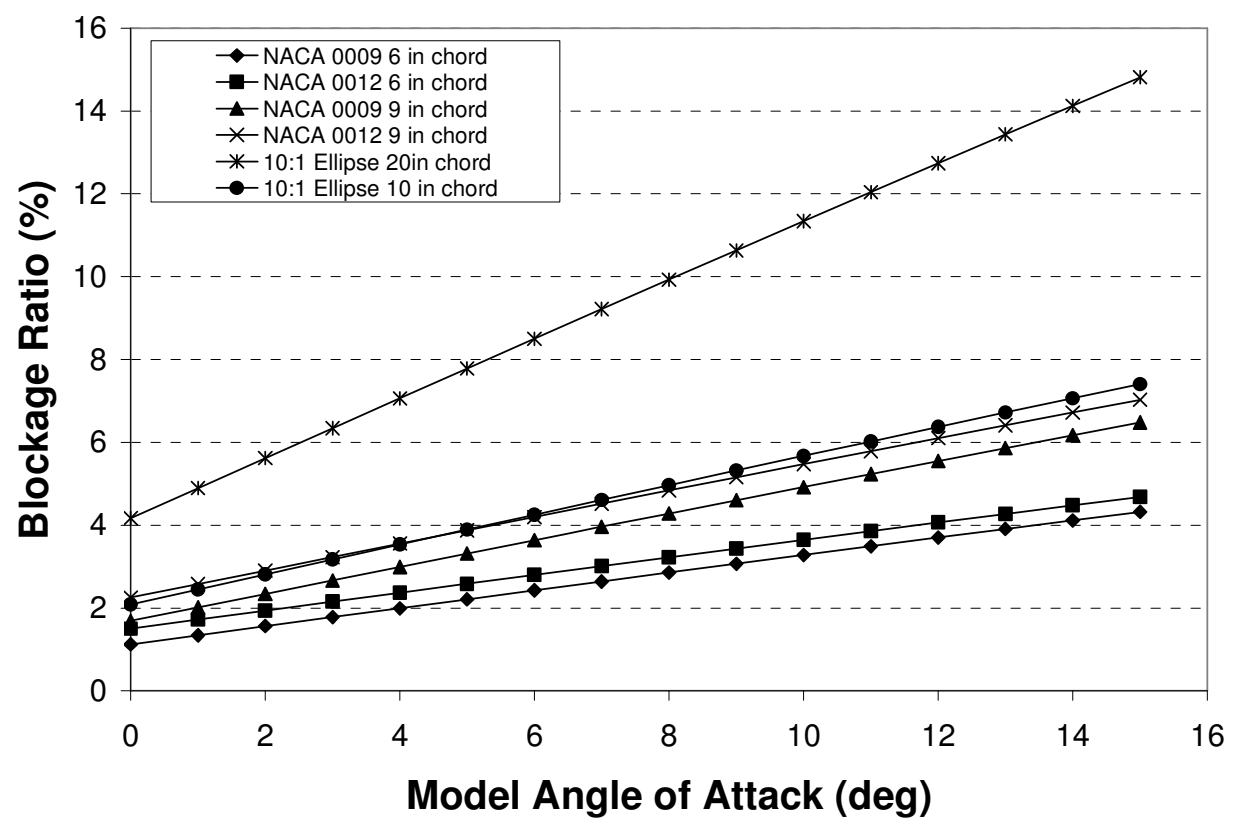

Figure 4.6: Blockage ratio in percent for basic airfoil shapes considered for the CCHB as dependent upon the angle of attack 


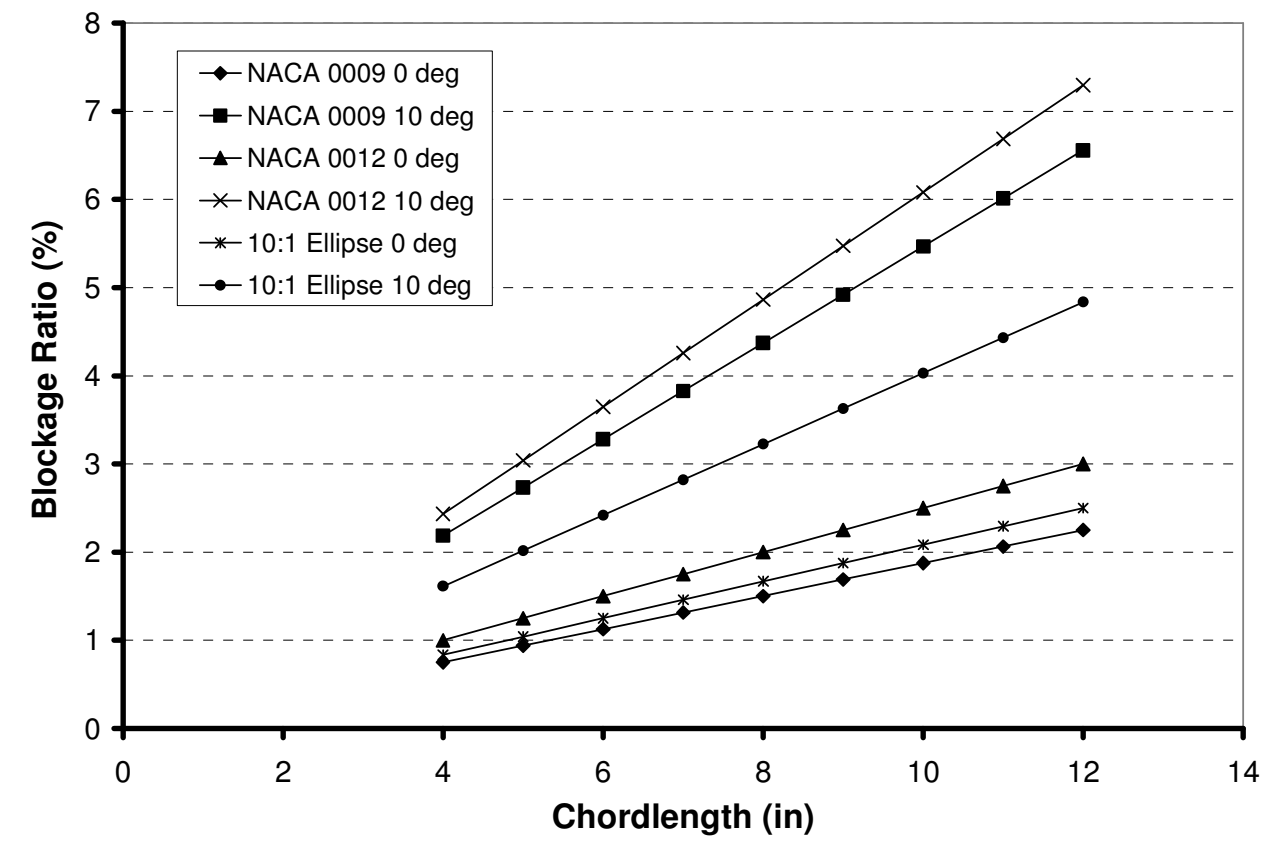

Figure 4.7: Blockage ratio in percent for basic airfoil shapes considered for the CCHB as dependent upon the chordlength

From these figures, it is evident that smaller the chordlengths result in the smallest blockage ratios, as expected. However, a small sized models places considerable tolerances on the manufacture of a model with circulation control, as well as restrictions on the supply of the airfoil to the blowing slots. Using the guideline for blockage ratio set by Barlow, Rae, and Pope [1999] of less than $7 \%$ the 10 inch chordlength elliptical airfoil was selected for testing in the $31 \frac{1}{2} 2$ inch by 45 inch test section of the Closed Loop Wind Tunnel. With this airfoil shape and size the blockage ratio was determined to reach the guideline of $7 \%$ at an angle of attack of 14 degrees. Barlow, Rae, and Pope [1999] also provide a correction factor for the drag force at relatively high blockage ratios, less than 20 percent, as discussed in Section 3.11. 


\subsection{Reynolds Number Analysis}

The Reynolds number as defined in Equation 4.4 was used to estimate the radial location, along the span of the rotor blade of the computational and experimental test conditions.

$$
\operatorname{Re}=\frac{\rho V c}{\mu}
$$

Equation 4.4

Here $\rho$ is the density of air, $\mathrm{V}$ is the resultant velocity (sum of the rotational speed and flight speed), $\mathrm{c}$ is the chord length and $\mu$ is the viscosity of air.

Using a helicopter rotor speed of $200 \mathrm{rpm}$, the rotational velocity and local velocities were determined for a 30 foot diameter (15 foot radius) with a chord length of one foot, helicopter rotor in hover and at flight speeds of $50 \mathrm{mph}$ and $100 \mathrm{mph}$. Figure 4.8 summarizes the data representative of the conditions conventional helicopter rotors are exposed to; the data is provided in Appendix B.

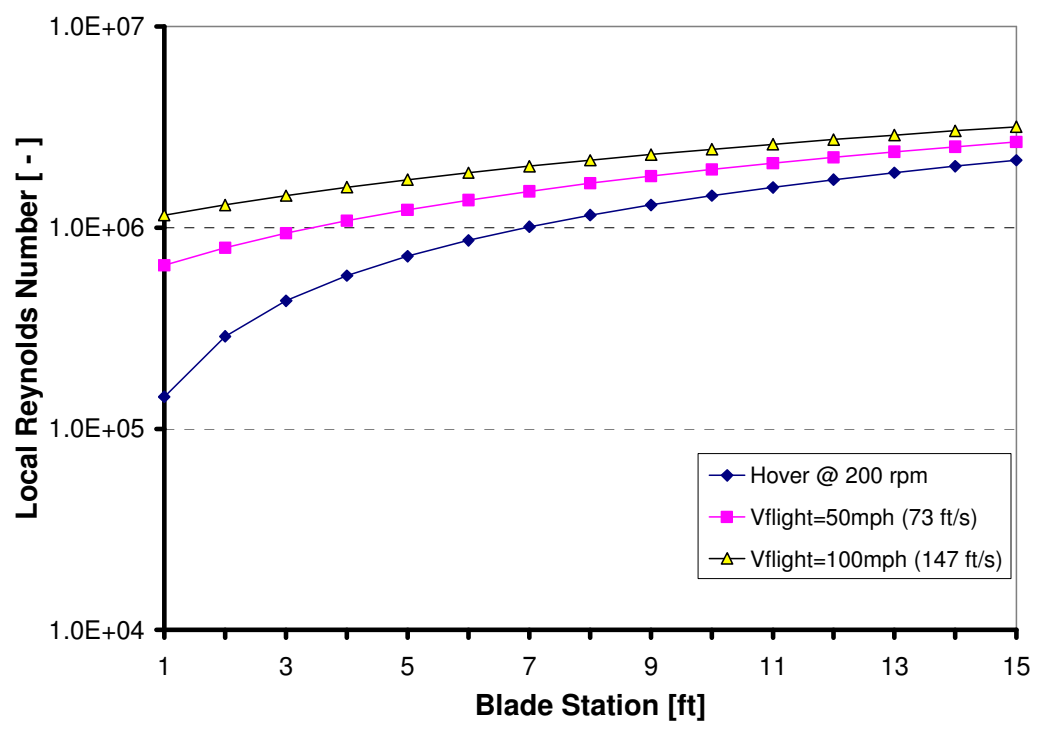

Figure 4.8: Local Reynolds number for a helicopter rotor, with a one foot chordlength, spinning at $200 \mathrm{rpm}$ as dependent upon the blade station 
The Reynolds numbers corresponding to the experimental conditions are summarized in Table 4.4 in which a radial station of 3 feet was simulated. The computational model was used to replicate larger radial stations and higher flight speeds as shown in Table 4.5 .

Table 4.4: Summary of the Reynolds numbers for the experimentally tested velocities and corresponding radius at flight speeds of $50 \mathrm{mph}$

\begin{tabular}{|c|c|c|}
\hline $\mathrm{V}$ & $\mathrm{RE}$ & Radius_V50mph \\
\hline $\mathrm{ft} / \mathrm{s}$ & - & $\mathrm{ft}$ \\
\hline 50 & $3.08 \mathrm{E}+05$ & 0.0 \\
\hline 75 & $4.62 \mathrm{E}+05$ & 0.0 \\
\hline 100 & $6.16 \mathrm{E}+05$ & 0.8 \\
\hline 125 & $7.70 \mathrm{E}+05$ & 1.8 \\
\hline 150 & $9.24 \mathrm{E}+05$ & 2.9 \\
\hline
\end{tabular}

Table 4.5: Summary of the Reynolds numbers from the computational study and corresponding radius at flight speeds of 500 and $100 \mathrm{mph}$

\begin{tabular}{|c|c|c|c|}
\hline$V$ & $R E$ & Radius_V50mph & Radius_V100mph \\
\hline $\mathrm{ft} / \mathrm{s}$ & - & $\mathrm{ft}$ & $\mathrm{ft}$ \\
\hline 80 & $4.93 \mathrm{E}+05$ & 0.0 & 0.0 \\
\hline 150 & $9.24 \mathrm{E}+05$ & 2.9 & 0.0 \\
\hline 450 & $2.77 \mathrm{E}+06$ & 15.7 & 12.3 \\
\hline 600 & $3.70 \mathrm{E}+06$ & 22.1 & 18.7 \\
\hline 750 & $4.62 \mathrm{E}+06$ & 28.5 & 25.1 \\
\hline 900 & $5.54 \mathrm{E}+06$ & 34.9 & 31.5 \\
\hline 1050 & $6.47 \mathrm{E}+06$ & 41.3 & 37.9 \\
\hline 1200 & $7.39 \mathrm{E}+06$ & 47.7 & 44.3 \\
\hline 1350 & $8.31 \mathrm{E}+06$ & 54.1 & 50.7 \\
\hline 1500 & $9.24 \mathrm{E}+06$ & 60.5 & 57.1 \\
\hline
\end{tabular}

\subsection{Summary}

This chapter provided background information that was developed to assist in the determination of experimental and computational model characteristics. As a result of the calculations for the response time characteristics, a goal of $60 \mathrm{msec}$ was selected for the development of the augmented forces to enable operation at current helicopter rotor speeds. The plenum filling time estimates revealed that $86 \mathrm{msec}$ was required just to fill the rotor blade for the application of hub controlled activation. This time requirement is outside the normal operational speeds of modern helicopter rotors, thereby eliminating 
the option of using a valve in the hub. The surface mounted valves of this work would improve the response characteristics of the aerodynamic forces, justifying the present research endeavor. The blockage and Reynolds number analyses lead to the sizing of the experimental and computational models used in this feasibility study. 


\section{Experimental Investigation}

The major objectives of this research effort have been to experimentally prove the response time of the circulation control and to determine the magnitude of change in aerodynamic characteristics created by the circulation control system of a 10:1 elliptical airfoil with forward and aft blowing slots. One model with appropriate instrumentation will be used to evaluate both aspects, though each aspect will be tested independently. To reduce model cost of this preliminary model, two rotating devices will be used to control the opening and closing of the blowing slots, for the leading and trailing edges, where an operational rotor blade would be expected to incorporate smart materials such as piezoelectric materials, or similar electronically controlled device, as the valve system.

Two models have been constructed for this testing. The two models have elliptic profiles with a rounded trailing edge, creating the Coanda surface. The airfoil is a 10:1 elliptical profile, with a 12 inch major diameter and a minor diameter of 1.2 inches; the trailing edge of the ellipse is trimmed by a radius of 0.4 inches, making the actual chord length of the model 11.8 inches and the span of the model is 31.5 inches. The models are made from machined aluminum and instrumented with surface pressure taps, and have attachments on the ends to connect to load cells that will be used for force determination.

\subsection{Experimental Apparatus}

\subsubsection{Baseline Model}

The CCHB airfoil shape is modified from a true elliptical airfoil, thus a baseline model was tested to determine the lift and drag characteristics of the elliptical shape with a trailing edge radius of 0.20 inches. Figure 5.1 shows the top view of the baseline model without the end plates. To ease the instrumentation process the model is constructed with a removable surface which allows access to the internals of the model. Figure 5.2 shows the internal structure of the model which contains a total of six ribs. The outer most ribs have a width of $3 / 4$ inches, the center two ribs have a width of $1 / 2$ inch and the intermediate ribs are $1 / 4$ inch wide. The trailing edge is removable and provides a clamping force to 
hold the upper surface in place, as shown in Figure 5.3. Figure 5.4 shows the end view of the model with the leading edge to the right, and with the upper surface fastened in place.

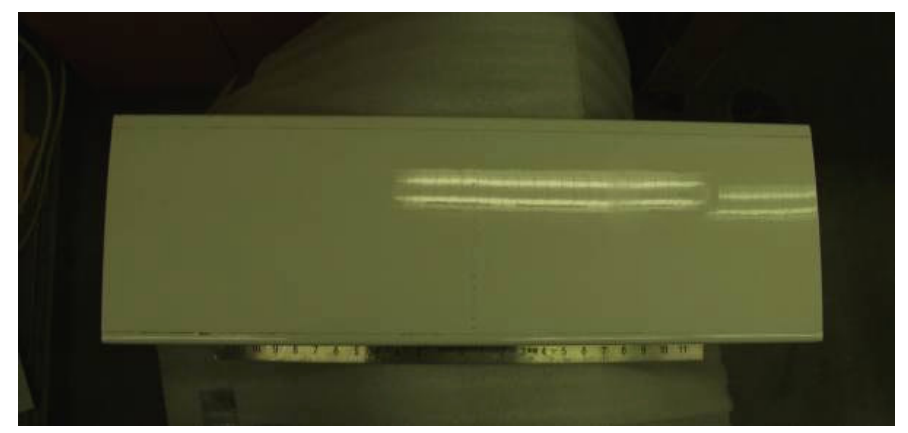

Figure 5.1: Top view of baseline elliptical airfoil model

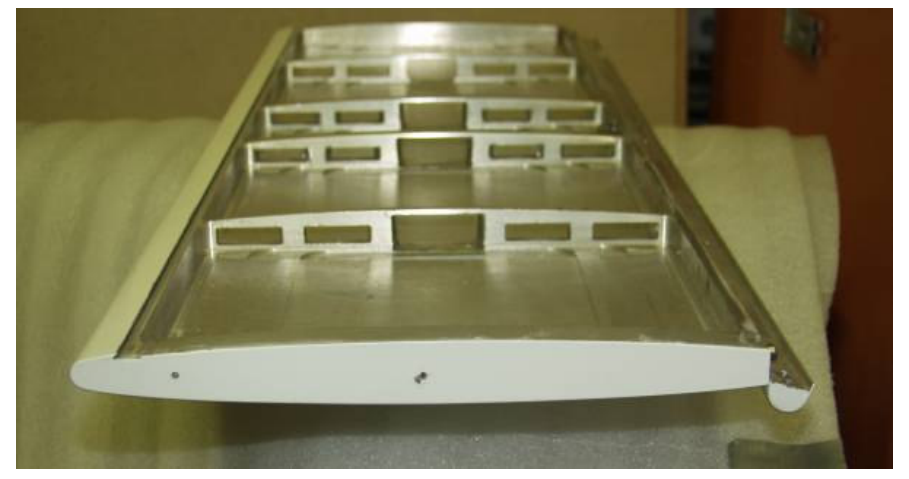

Figure 5.2: End view of baseline elliptic airfoil model with top cover and trailing edge removed

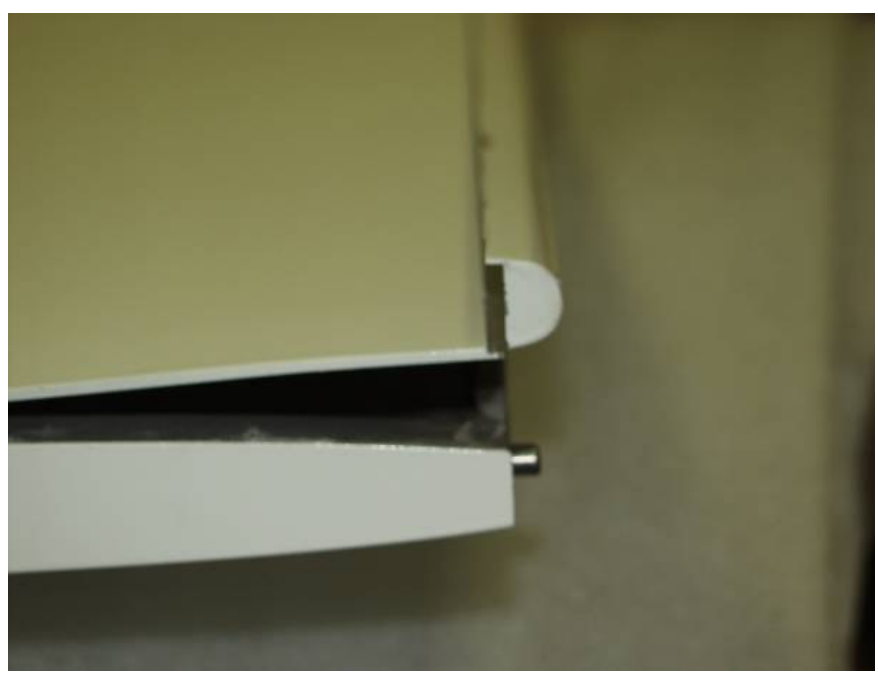

Figure 5.3: Close-up view of the assembly method for the baseline elliptic airfoil model 


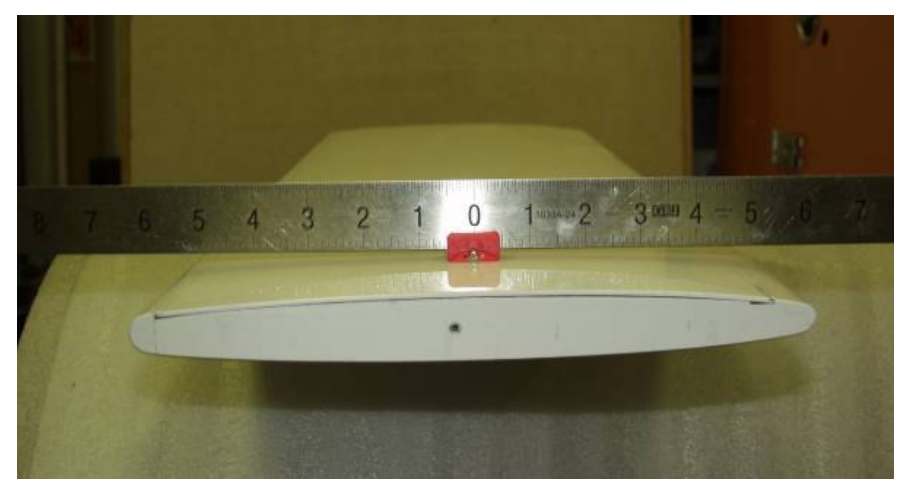

Figure 5.4: End view of assembled baseline elliptic airfoil model without endplates

Surface pressure taps have been distributed across the centerline of the upper surface of the model, Figure 5.5, with additional taps machined into the leading and trailing edges. The surface pressure reading allowed for a second measurement technique of the primary aerodynamic forces of lift and drag. The pressure taps will be focused on one side of the model, the positions of the upper surface pressure taps are listed in Table 5.1. The concentration of the surface pressure taps on one side of the model is to increase the resolution of the measurement with existing instrumentation capabilities. The use of a symmetric airfoil allows for upper and lower surface measurements when testing at positive and negative angles of attack.

Endplates were attached to the Baseline Model such that their vertical spacing was the same as that of the Active Circulation Control Model, described in Section 5.1.2. The baseline model installed in the WVU low-speed wind tunnel for testing is shown in Figure 5.6. 


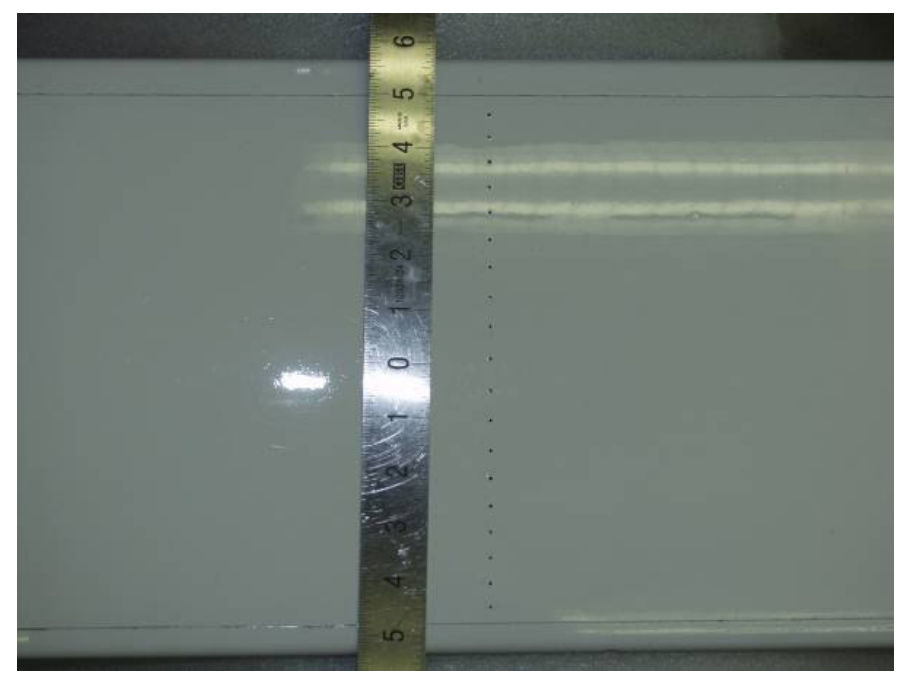

Figure 5.5: Close-up of surface pressure tap spacing along top surface of the baseline elliptic airfoil model

Table 5.1: Surface pressure tap locations, in the chordwise direction on the upper skin

\begin{tabular}{|c|c|c|}
\hline $\begin{array}{c}\text { Tap } \\
\text { Number }\end{array}$ & \multicolumn{2}{|c|}{$\begin{array}{c}\text { Location from } \\
\text { Leading Edge } \\
\text { of Skin }\end{array}$} \\
\hline- & $\mathrm{mm}$ & in \\
\hline 1 & 14.5 & 0.571 \\
\hline 2 & 26.5 & 1.043 \\
\hline 3 & 40 & 1.575 \\
\hline 4 & 52.5 & 2.067 \\
\hline 5 & 65 & 2.559 \\
\hline 6 & 77.5 & 3.051 \\
\hline 7 & 90 & 3.543 \\
\hline 8 & 102.5 & 4.035 \\
\hline 9 & 115 & 4.528 \\
\hline 10 & 128 & 5.039 \\
\hline 11 & 141 & 5.551 \\
\hline 12 & 153 & 6.024 \\
\hline 13 & 166 & 6.535 \\
\hline 14 & 179.5 & 7.067 \\
\hline 15 & 192 & 7.559 \\
\hline 16 & 204.5 & 8.051 \\
\hline 17 & 218 & 8.583 \\
\hline 18 & 230.5 & 9.075 \\
\hline 19 & 242 & 9.528 \\
\hline \multicolumn{2}{|r}{}
\end{tabular}




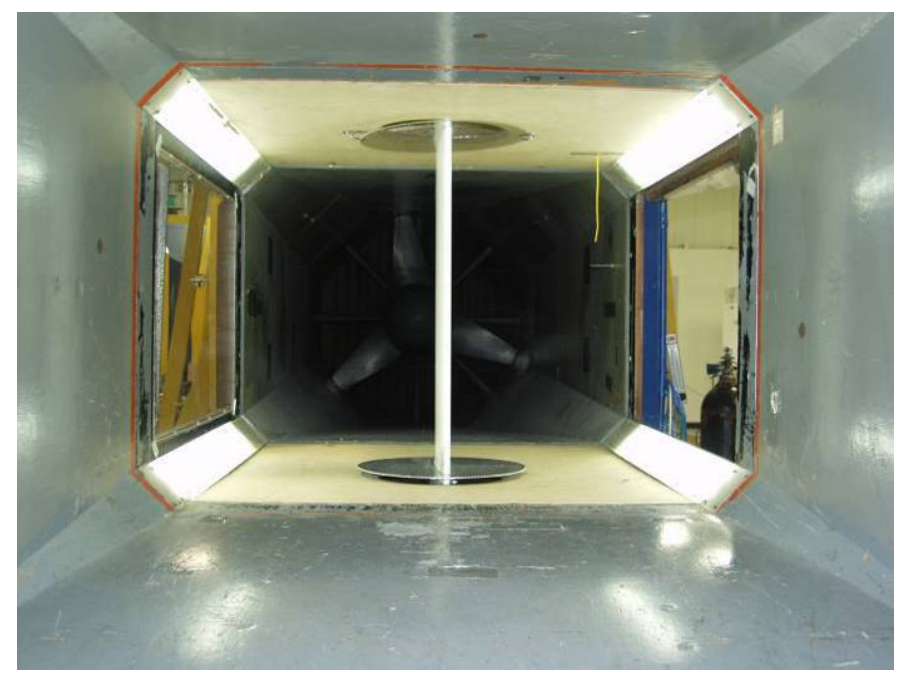

Figure 5.6: Baseline model installed in the WVU Closed Loop Wind Tunnel

\subsubsection{Active Circulation Control Model}

The second model used the same elliptical airfoil with an aspect ratio of 10:1, Figure 5.7. The two-dimensional model Figure 5.8, was built with a chord length of 11.8 inches and a maximum thickness of 1.2 inches, but with a span of 31.5 inches. The airfoil has been divided into four plenums for circulation control supply air distribution, arranged as shown in Figure 5.9. The two supply air lines enter the model from the ceiling of the wind tunnel, near the center of the airfoil for the leading and trailing edge plenums of the model respectively, which are separated by a solid wall. The entrance plenum is connected to a second plenum for both leading and trailing edges through a porous wall, which creates a pressure drop, assisting in the stabilization of a uniform slot exit pressure profile.

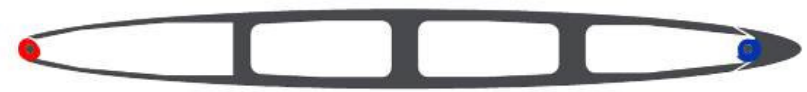

Figure 5.7: Cross-sectional schematic of 10:1 elliptic airfoil with internal plenums 


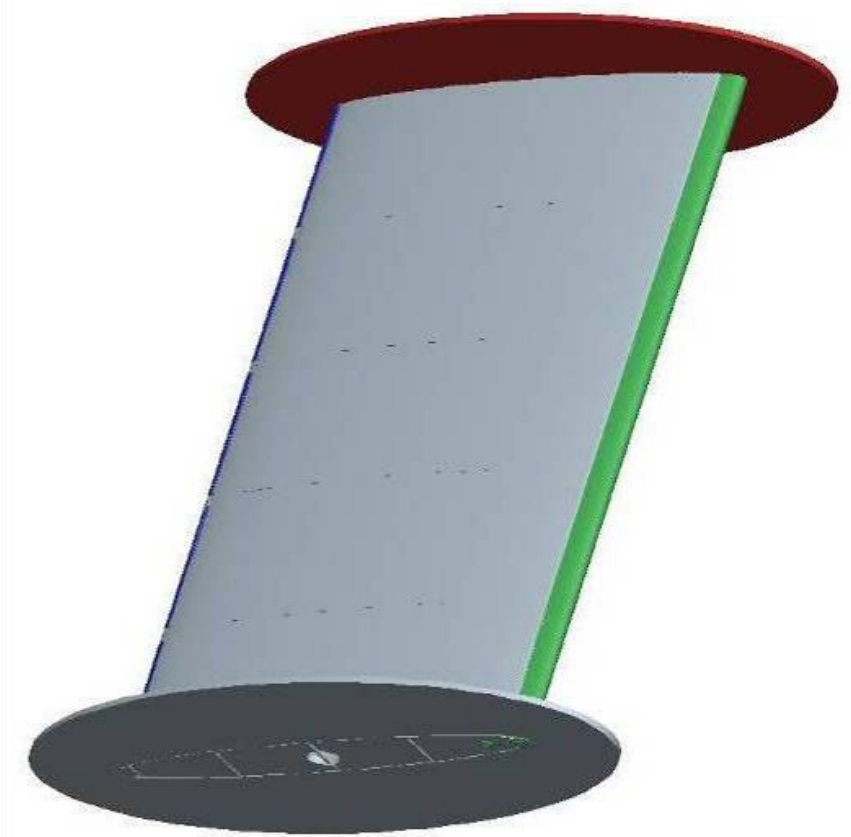

Figure 5.8: Two-dimensional model of circulation controlled helicopter blade with endplates

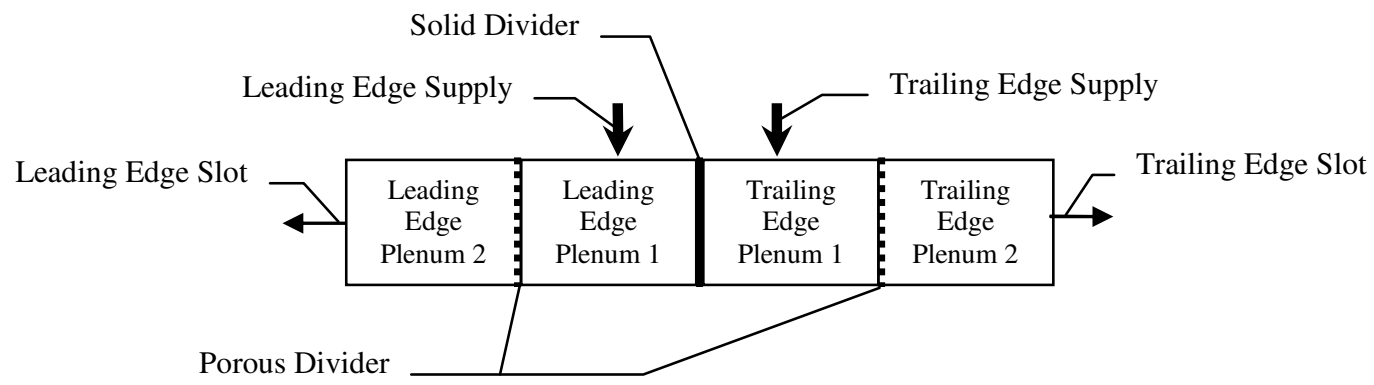

Figure 5.9: Block diagram of internal airflow of wind tunnel model

The development of the leading and trailing edge rotating device is an important component of this model. These devices provide seals when in the closed position which can be opened rapidly to simulate the use of smart materials, such as piezoelectronics, at the slot opening.

The speed at which the device opens and closes influences the response of the free stream to the blowing slot. Requiring the smallest possible rotation of the device is one way to reduce the time required to open or close. The initial configuration of the leading edge rotating device, Figure 5.10, requires approximately 2 degrees of rotation to fully open or close one slot. Figure 5.11 illustrates the second concept of the leading edge slot 
shape. Both of these configurations slightly alter the leading edge shape, which effectively creates a leading edge droop, when the upper slot is opened. This small change in airfoil shape could potentially be useful in enhancing the lift of the overall blade; however, for this preliminary investigation it was desired to minimize the changes to the blades overall shape to determine the effects of the circulation control system. A second drawback to using the shapes shown in Figure 5.10 and Figure 5.11 is the difficulty of sealing the system when both slots are desired to be closed. This led to the final configuration of the leading edge device, shown in Figure 5.12 which contains a fixed leading edge and provides the ability to close both the upper and the lower blowing slots at the same time. The final rotary valve configuration is symmetric with small ridges on each side to assist in deflecting the airflow from the plenum to the blowing slot.

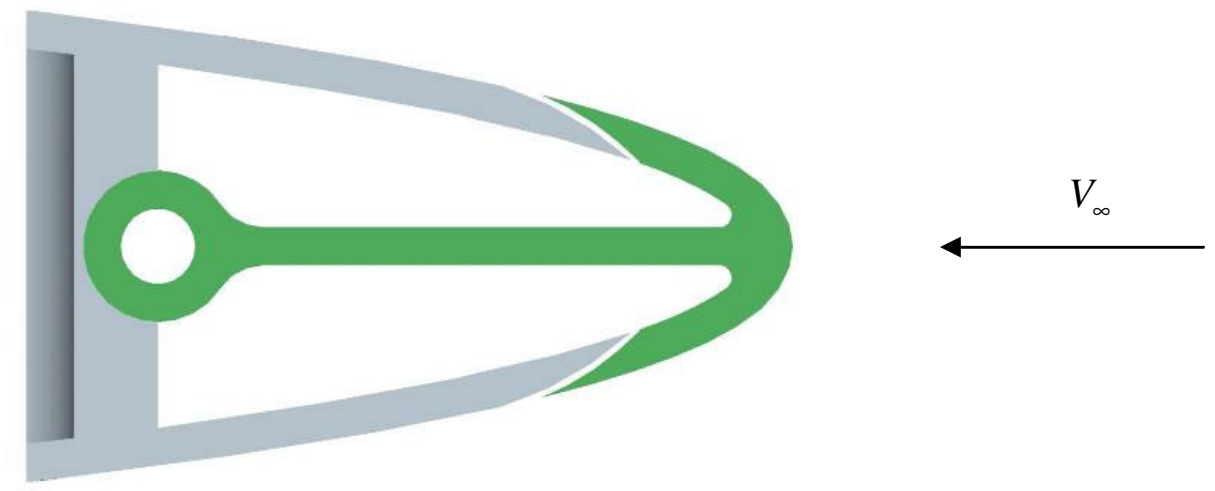

Figure 5.10: Preliminary leading edge slot control configuration for wind tunnel model

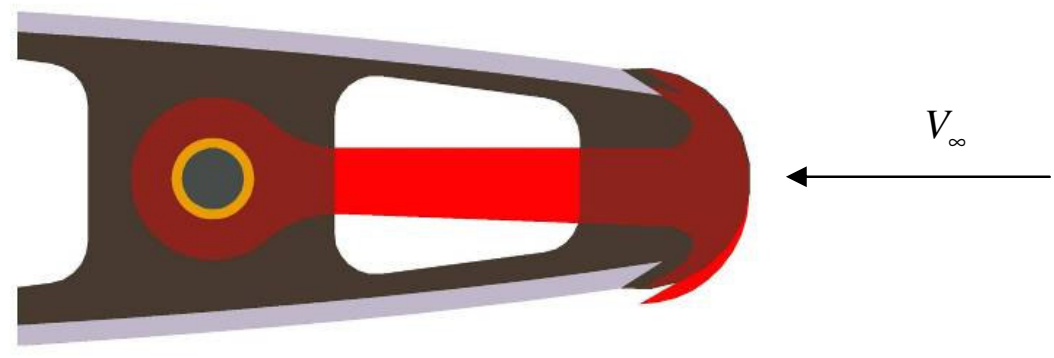

Figure 5.11: Second leading edge slot control configuration for wind tunnel model 


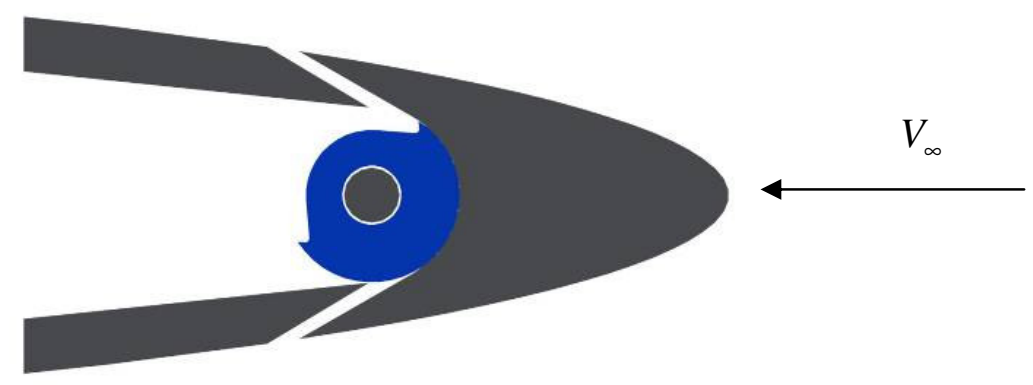

Figure 5.12: Final leading edge slot control configuration for wind tunnel model

A similar development process was conducted for the trailing edge slot control device. The final slot configuration of the trailing edge, shown in Figure 5.13, incorporates a profile of the radius which transitions from a minimum toward the plenum to the trailing edge radius within 90 degrees. The radial profile of the trailing edge devices considered during the design are compared in Figure 5.14 where the angle $\theta$ is defined as the displacement from the centered position, with the positive direction being that required to open the upper surface blowing slot. The remaining portion of the device $(90<\theta<180)$ is at the maximum radius and acts as the Coanda surface for the trailing edge blowing slot.
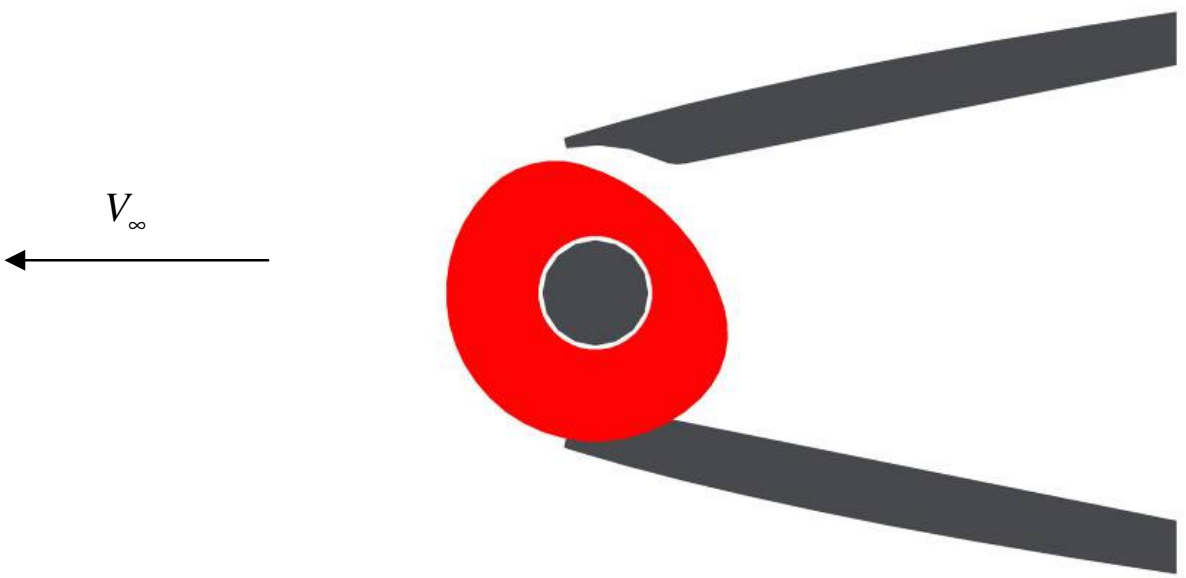

Figure 5.13: Final trailing edge slot control configuration for wind tunnel model 


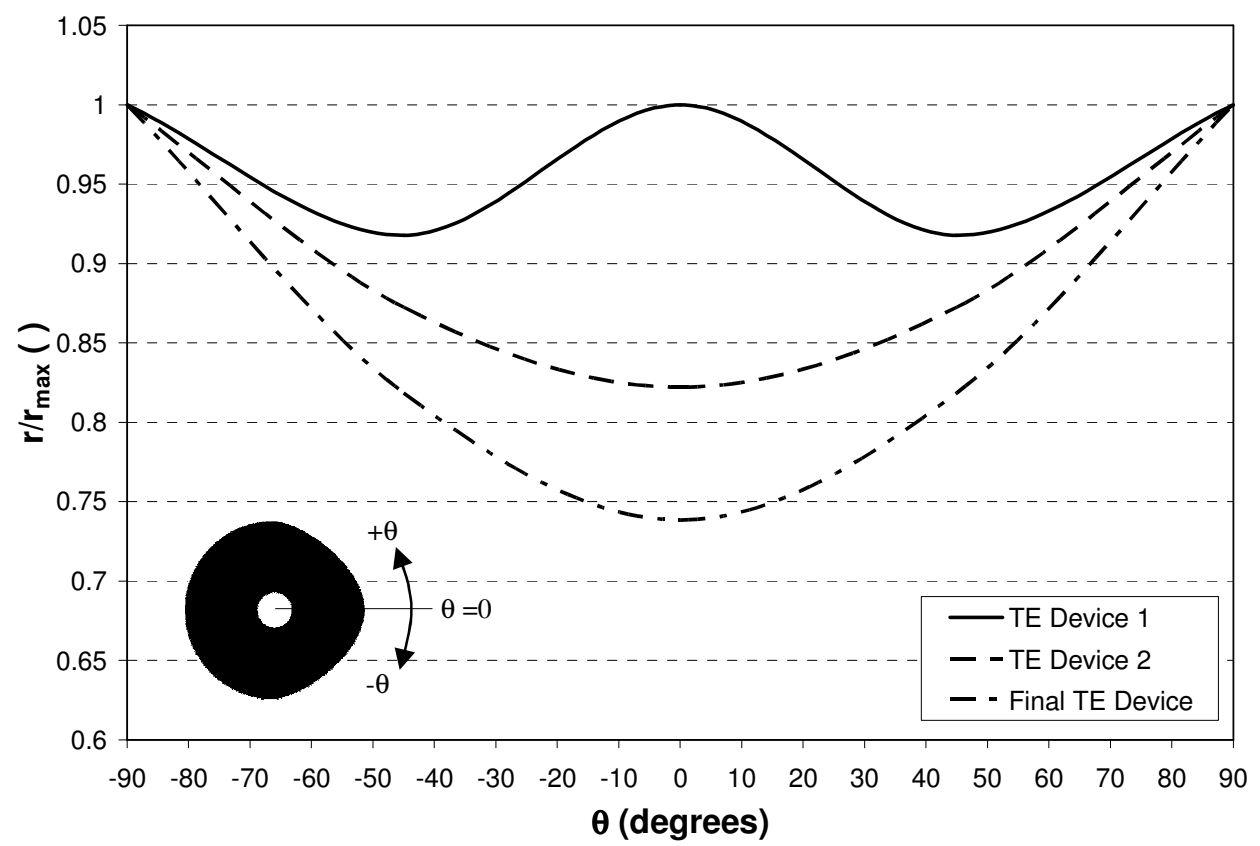

Figure 5.14: Profile of radius for the three trailing edge slot control devices

The structure of the active circulation control model is similar to that of the baseline model. It contains six ribs, all of which have a width of half an inch and both the upper and lower surfaces were removable. The rotating devices are installed in the model through the channels in the ribs for the circulation control air and held in place with a bearing surface and set screws. The ribs and add-on surfaces created the bearings for the rotating device and are coated with Teflon to decrease the friction between the aluminum surfaces and the aluminum trailing edge rotating valve.

Solenoids were used to control the rotating valves that opened the leading and trailing edge blowing slots. Figure 5.15 shows the setup of the solenoids used to control the leading edge valve. This particular setup was designed to open and close one side of the rotary valve (upper surface); thus the set point on the rotary shaft was changed to control the opposite slot (lower surface). The activation of the solenoids was controlled by the data acquisition software, and opened in 0.055 seconds ( 55 milliseconds). 


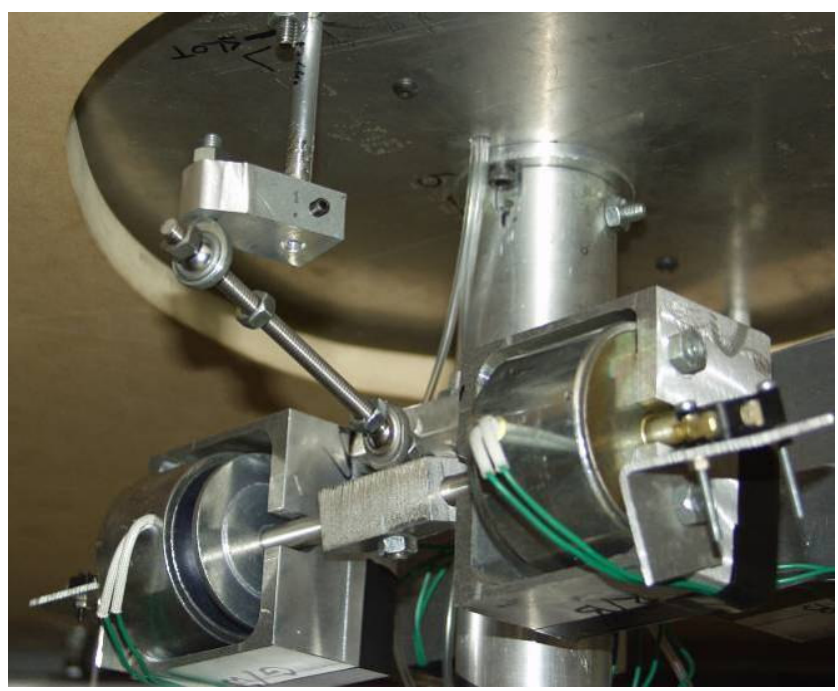

Figure 5.15: Leading edge solenoid control configuration for the rotary valve

The air pressure inlets, as well as the porous walls dividing the first and second plenums are shown in Figure 5.16. The tubing for the surface pressure taps was held in place with silicone against the center divider and the inner side of the lower surface of the model, exiting the model at the bottom as shown in Figure 5.17. A thin rubber strip was adhered to the top of the center divider to seal the pressure plenums (leading and trailing) from each other. A silicone bead was placed between the lower surface and the center divider completing the seal between the leading and trailing edge plenums.

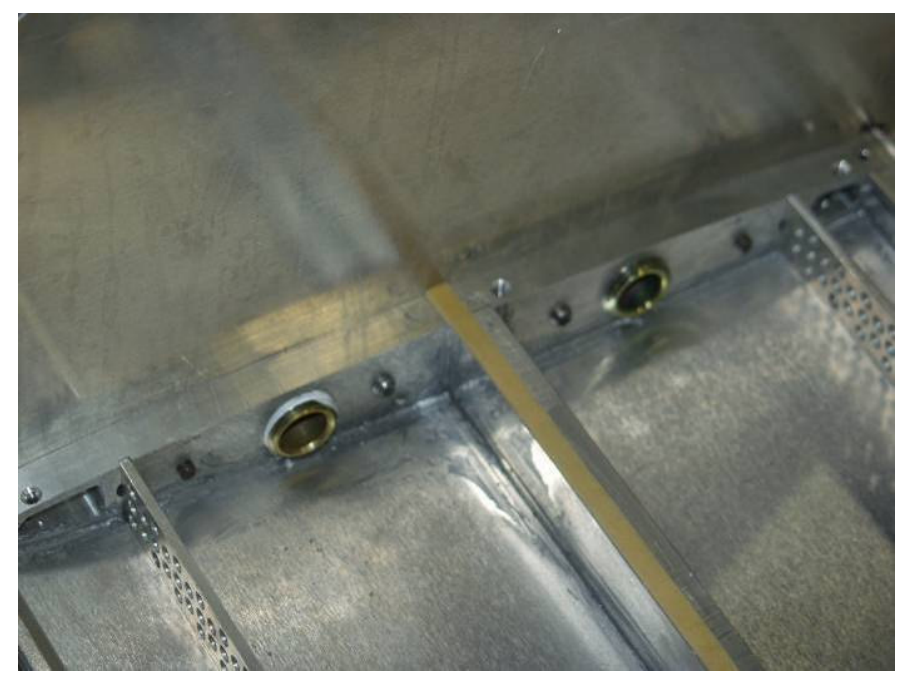

Figure 5.16: Close up of CCHB model internal components, including the air inlets, and porous walls 


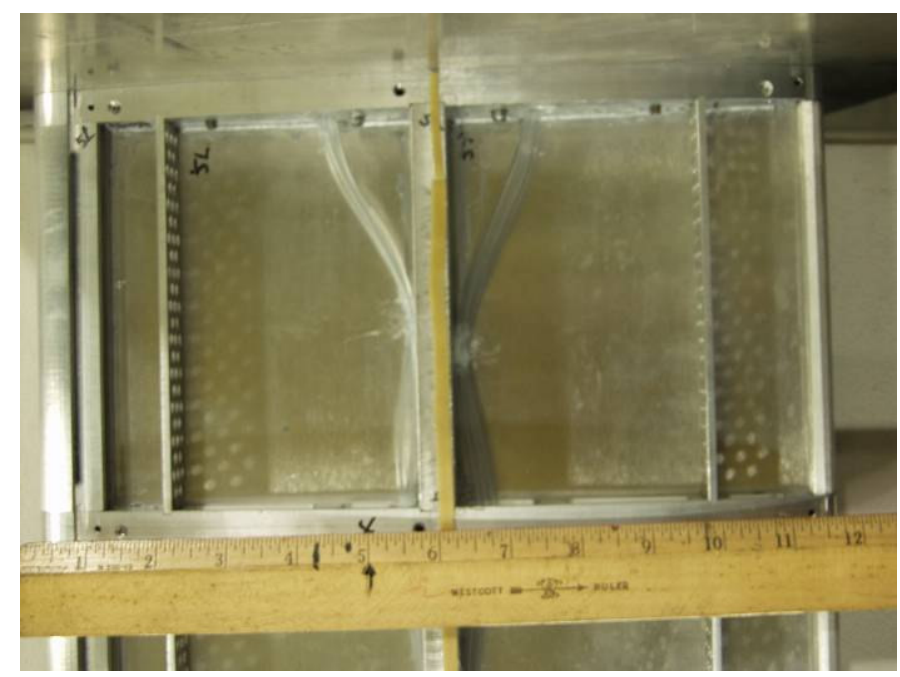

Figure 5.17: Chordwise view of the CCHB model showing the leading edge slot structure and trailing edge rotating device

\subsection{Experimental Instrumentation}

The measurement system for both the baseline elliptic airfoil and the Circulation Control Helicopter Blade models was the same. Of the two characteristics to be proven, the testing for the magnitude of change in the force measurement is the least complex to determine. Care was taken in determining the speed at which the freestream responds to the introduction of circulation control blowing, since the speed at which the slot itself opens influences the response of the freestream.

The method used to measure the lift and drag was through the use of load cell(s). The load cell configuration, partially shown Figure 5.18, contained a series of four load cells used to record the aerodynamic forces of lift and drag on both ends (top and bottom) of the model. Placed between the load cells and the model were two aluminum discs, one which rotated with the model, with the other pinned to a fixed plate connected to the load cells. A hole pattern, consisting of two degree increments from -4 to 16 degrees, was placed in the rotating disc, and pinned to the stationary disc to repeatably set the model at different angles of attack, as shown in Figure 5.19. 


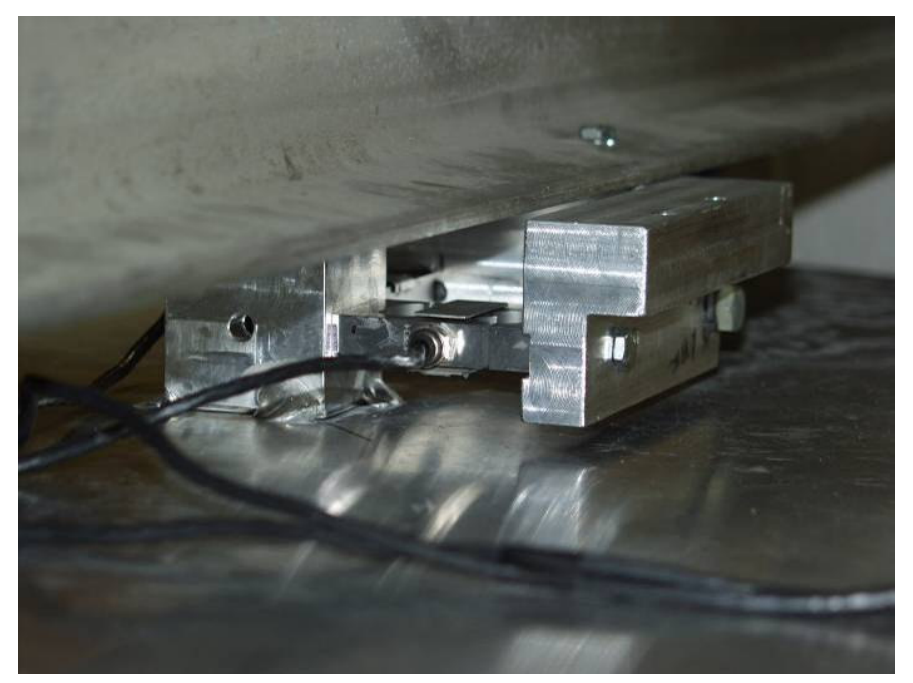

Figure 5.18: Upper load cell configuration

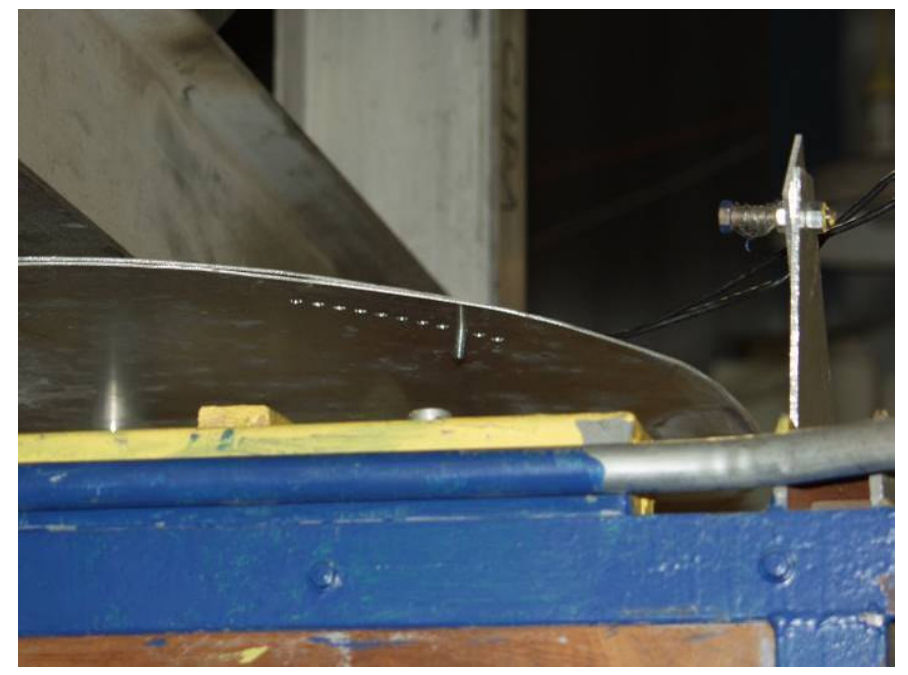

Figure 5.19: Angle of attack setting device pinned at 0 degree, indicated angle of attack

\subsection{Load Cell Calibration}

Measurement devices, especially electric ones, require calibration to ensure that accurate values are recorded. The load cells were calibrated after each model (baseline and active) was installed in the test section by applying known forces via weights and pulleys to the model. Figure 5.20 shows a configuration used to apply a lift force on the model. In addition to applying forces along the center of the model as shown, forces were also applied at the connection point of the model to the load cells, shown in Figure 5.21, 
allowing for a calibration curve to be created for each load cell, to determine the interference between load cells.

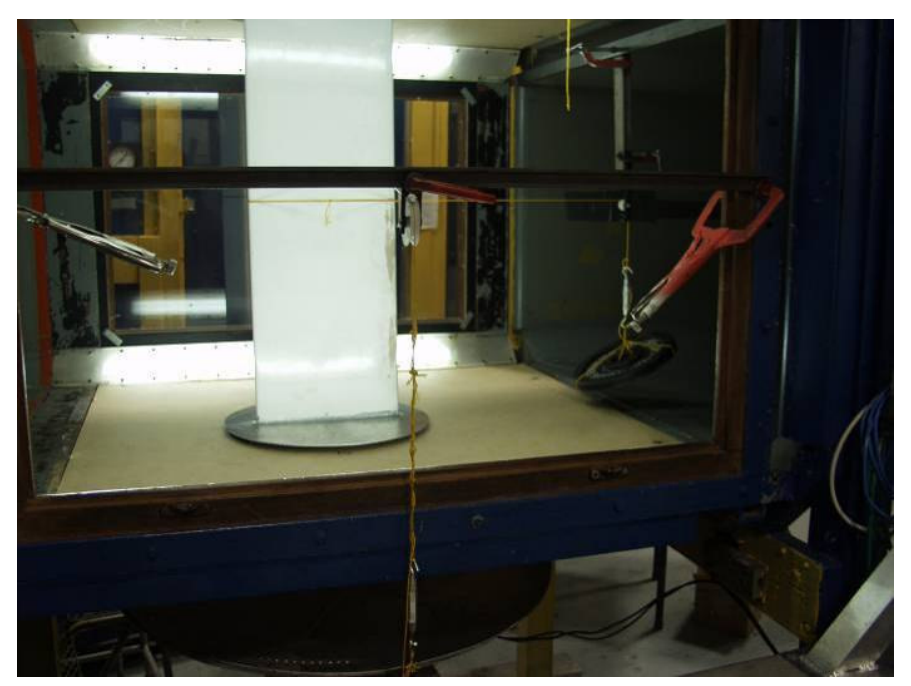

Figure 5.20: Centerline calibration setup of the baseline model

By applying a series of weights in the direction of the lift and drag forces (normal to and with the freestream) and recording the corresponding voltages a relationship between the analog voltage recorded and force was made. Figure 5.21 shows the calibration curves for the four load cells (top lift, TL, top drag, TD, bottom lift, BL, and bottom drag, BD), calibration curves with the baseline model. The calibration curves for the active circulation control model are shown in Appendix B. The rigid connection between the lift and drag load cells created some interference between the two measurements. The relationship between the measured lift and drag and the error in the perpendicular measurement was found by applying combinations of loads and was estimated with the linear fit to the data shown in Figure 5.22. This error was found to be considerably large and is due to the direct linkage between the lift and drag directions. The linkage transferred a portion of the lift force to the drag through the torque at the connection point and was compensated for during the data reduction. 
A.
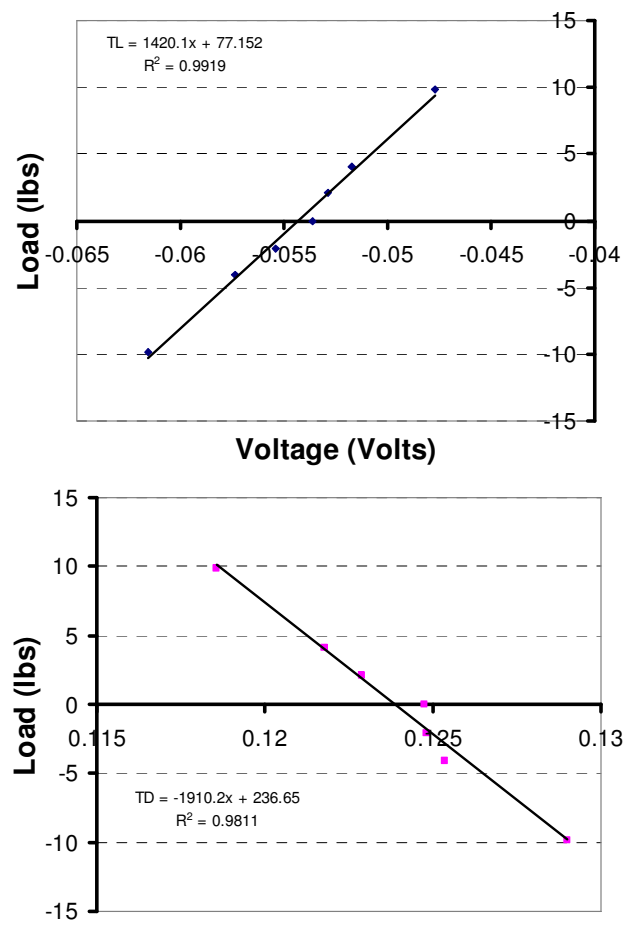

B.

Voltage (Volts)

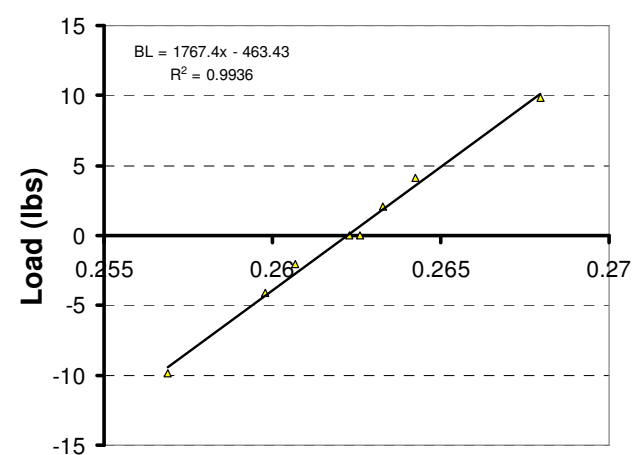

C.

Voltage (Volts)

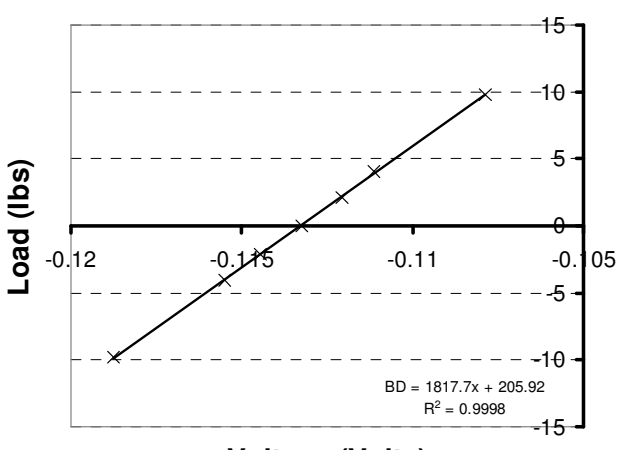

Voltage (Volts)

Figure 5.21: Calibrations of the four load cells with the baseline model:
A. Top Lift
C. Bottom Lift
B. Top Drag
D. Bottom Drag

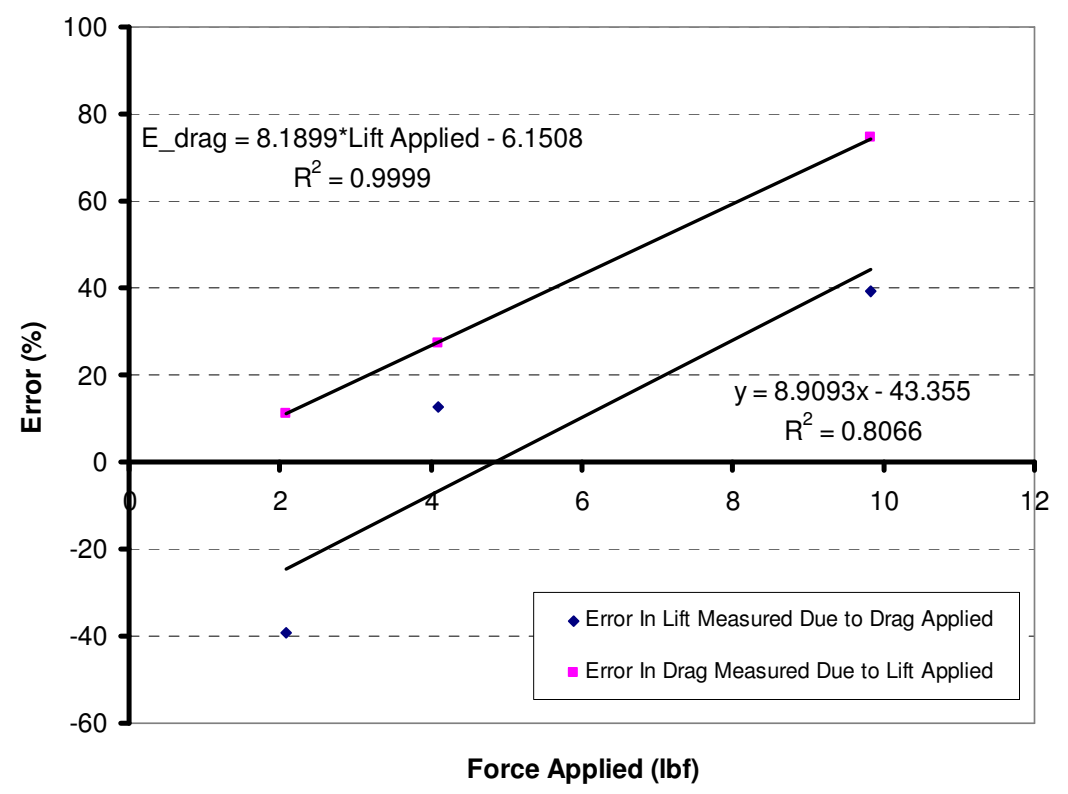

Figure 5.22: Interference of force measurement due to the opposite force 


\subsection{Force Response of Rotating Valve Actuation}

In order to effectively predict the forces experienced by the model, all of the forces occurring during and immediately after activating the solenoids were recorded. An example of the forces experienced with no freestream velocity during the activation of the solenoids were recorded and are shown as a function of time in Figure 5.23 for bottom drag force due to the opening of both the leading and trailing edge blowing slots. The bottom drag force was also recorded during the activation of only the leading edge blowing slot and is shown in Figure 5.24, where Figure 5.25 shows the bottom drag forces from the trailing edge slot activation. Since the amplitude and the damping rate of the forces created by the solenoids were consistent for the repeat runs (Case \#1, Case \#2, and Case \#3), within $~ 2 \%$, these forces were tared out of the data collected during the dynamic, time response tests.

As seen in the following figures, the activation of the solenoids induces a dynamic response which damps out in approximately one second. Similar graphs are shown in Appendix D for the dynamic response of the lift force to opening of the valves with various experimental blowing configurations. In the determination of the time response of the circulation control system, the forces created by the activation of the solenoids were removed as tare values from the data.

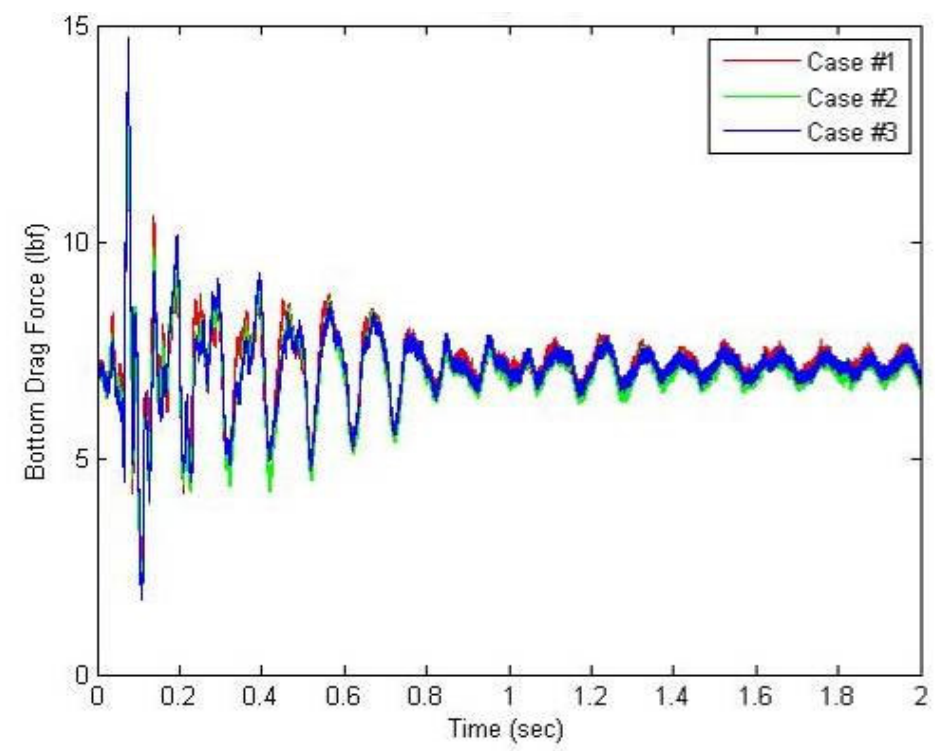

Figure 5.23: Measured forces during solenoid opening of both blowing slots in bottom drag with no freestream velocity 


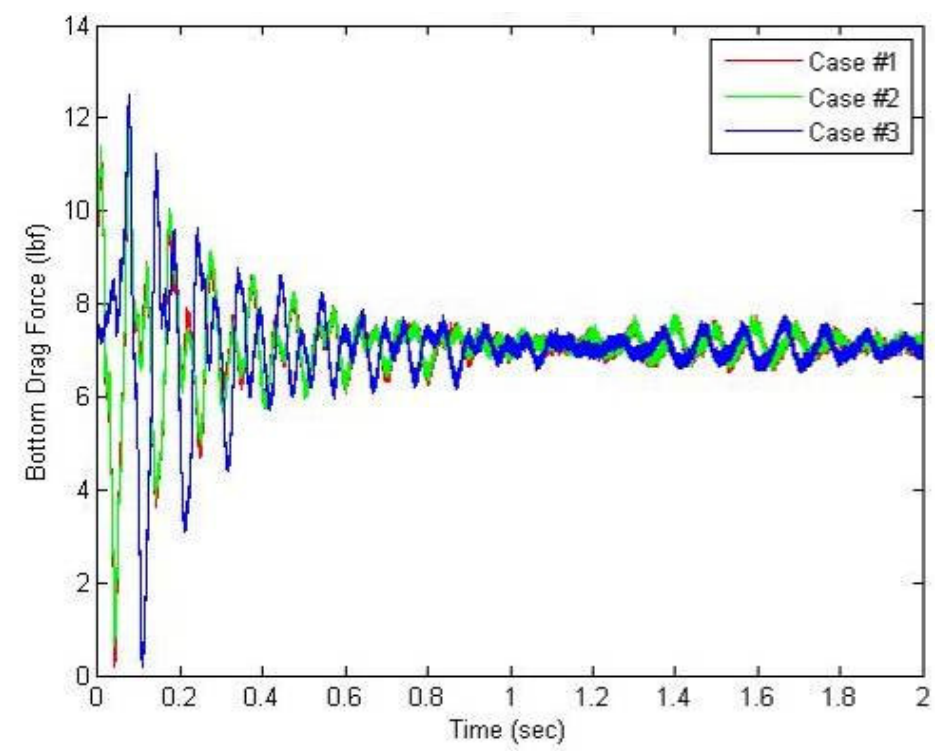

Figure 5.24: Measured forces during solenoid opening of leading edge blowing slot in bottom drag with no freestream velocity

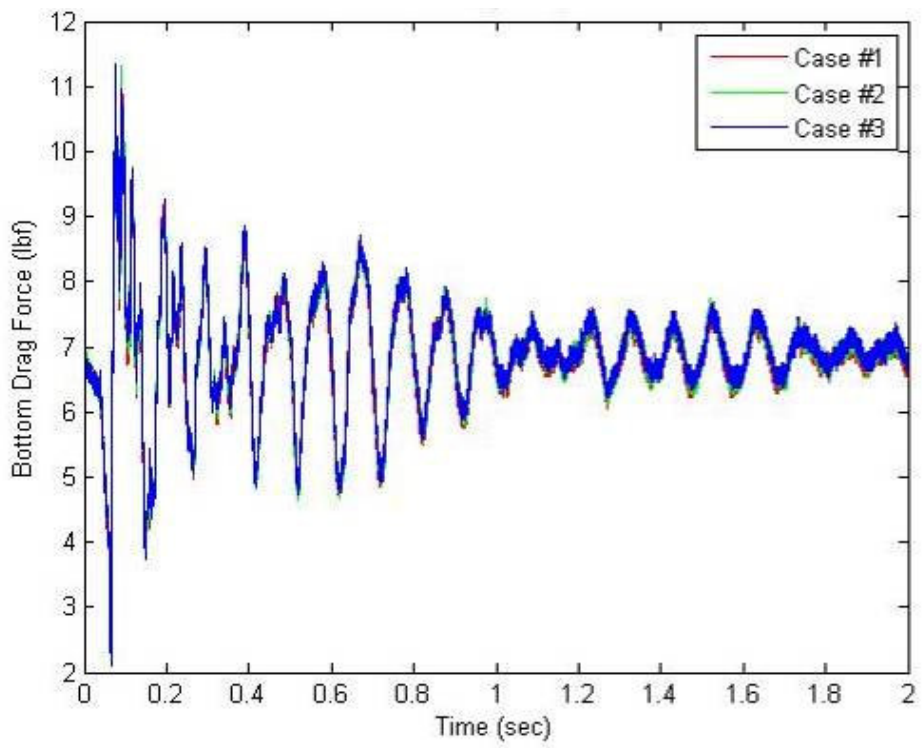

Figure 5.25: Measured forces during solenoid opening of trailing edge blowing slot in bottom drag with no freestream velocity

\subsection{Blowing Slot Velocity Profiles}

The velocity profiles of the circulation control blowing slots were measured to verify the two-dimensional nature of the active model which were then nondimensionalized by dividing by the average jet velocity at that particular blowing condition. The blowing slot velocity exit profiles were measured with a pitot-static probe 
and are shown in Figure 5.26 and Figure 5.27 for the leading and trailing edge slots, respectively. During the measurement of the exit profiles it was noticed that the blowing slot at the top of the model, near the inlet of the pressure system, was restricted and no reliably measurable velocity was present in the first six inches of the model for both the leading and trailing edge. This alteration of the span of the blowing slot was accounted for in the determination of the blowing coefficient.

As seen in Figure 5.26, the non-dimensional velocity profile for the leading edge blowing slot has a relatively low region near the center of the model and the velocity increases toward the bottom end plate of the model to a value for $V / \mathrm{V}_{\text {avg }}$ of approximately 1.1. The profile of the trailing edge velocity, Figure 5.27, was found to be more uniform with a high velocity region $\left(\mathrm{V} / \mathrm{V}_{\mathrm{avg}} \sim 1.08\right)$ near the models' center. Since the trailing edge is the primary interest for lift augmentation, these profiles were deemed reasonable to continue without modification to the model.

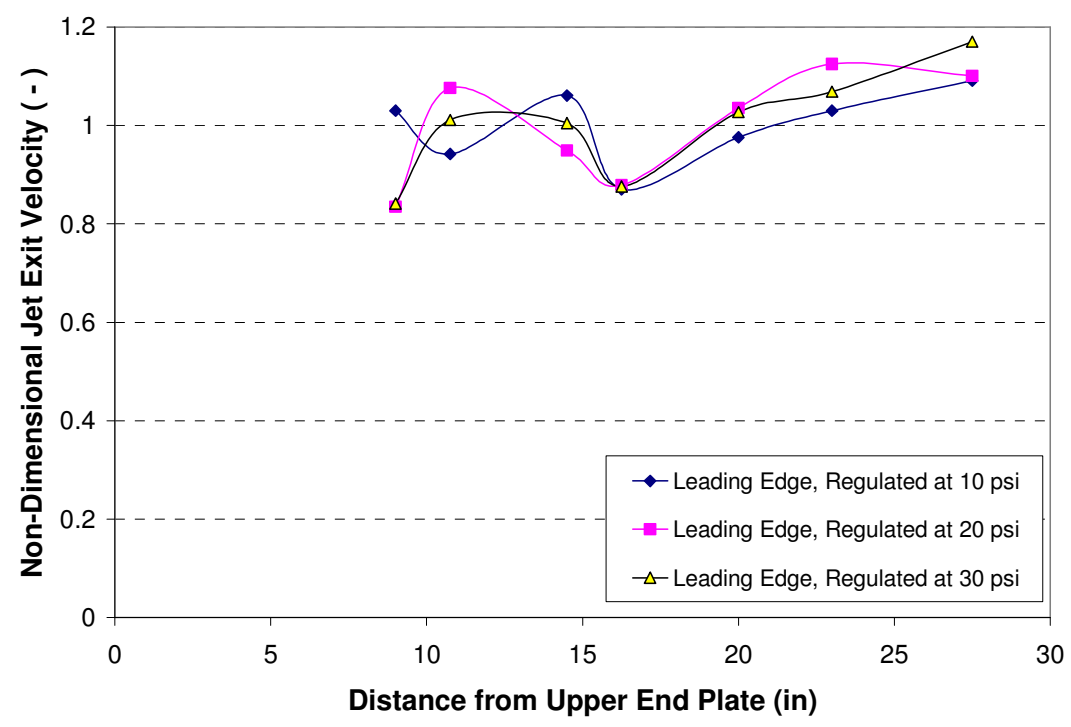

Figure 5.26: Non-dimensional blowing slot exit profile for the leading edge 


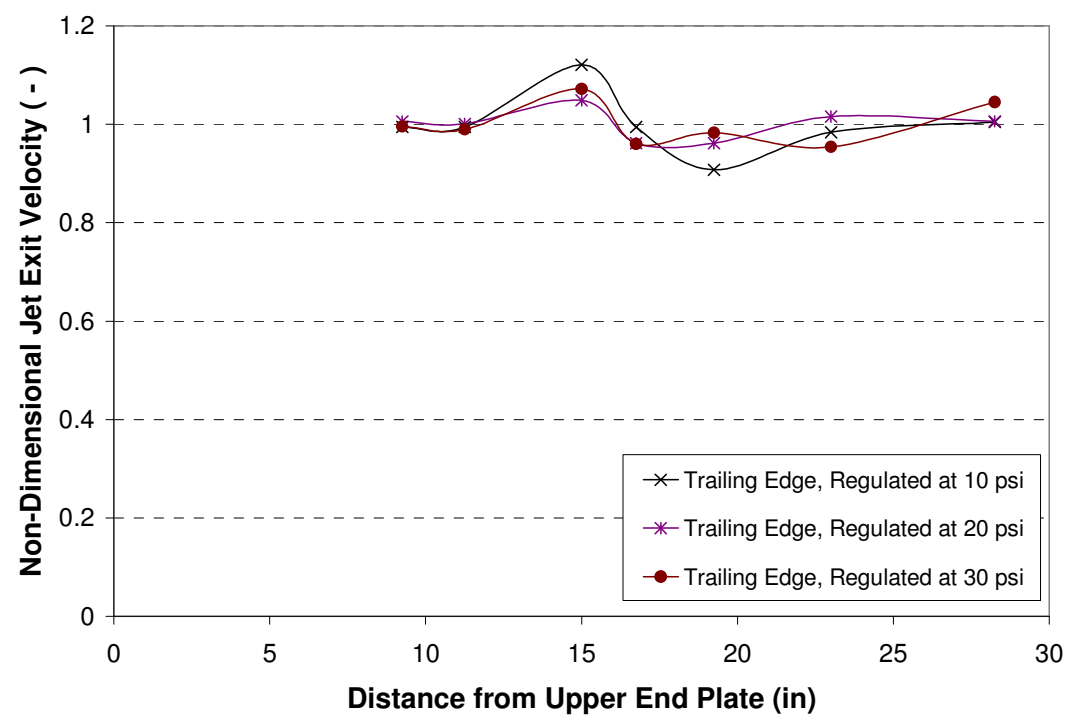

Figure 5.27: Non-dimensional blowing slot exit profile for the trailing edge

\subsection{Baseline Model Experimental Testing}

Testing was conducted in the West Virginia University Closed Loop Wind Tunnel's 3 foot by 4 foot test section. By testing at different wind speeds, the velocity independence nature of the circulation control airfoil shape was investigated. The wind speeds tested during this part of the experimental phase were $80 \mathrm{ft} / \mathrm{s}, 100 \mathrm{ft} / \mathrm{s}$, and 150 $\mathrm{ft} / \mathrm{s}$. The baseline model was tested at various angles of attack ranging from -4 to 16 degrees, in increments of two degrees, to determine the lift curve for the modified elliptical airfoil shape. The angles of attack were set for the 11 cases as shown in Table 5.2. Experimental runs 1 through 9 were collected at approximately $100 \mathrm{ft} / \mathrm{s}$, Run 10 was taken at $80 \mathrm{ft} / \mathrm{s}$ and Run 11 was taken at $150 \mathrm{ft} / \mathrm{s}$. The reduced data from the individual tests in both engineering units and in coefficient form are provided in Appendix E. 
Table 5.2: Angle of attack matrix used for baseline model testing

\begin{tabular}{|c|c|c|c|c|c|c|c|c|c|c|c|c|}
\cline { 2 - 16 } \multicolumn{1}{c|}{} & \multicolumn{10}{c|}{ Angle of Attack } \\
\hline Test Case & Run 1 & Run 2 & Run 3 & Run 4 & Run 5 & Run 6 & Run 7 & Run 8 & Run 9 & Run 10 & Run 11 \\
\hline 1 & -4 & -4 & -4 & -4 & 6 & 6 & 6 & -4 & 6 & -2 & -4 \\
\hline 2 & -2 & 16 & 8 & 8 & 8 & 16 & 16 & 16 & 8 & 8 & 8 \\
\hline 3 & 0 & -2 & -2 & -2 & 4 & 4 & 4 & -2 & 4 & -4 & -2 \\
\hline 4 & 2 & 14 & 10 & 10 & 10 & 14 & 14 & 14 & 10 & 10 & 10 \\
\hline 5 & 4 & 0 & 0 & 0 & 2 & 2 & 2 & 0 & 2 & 0 & 0 \\
\hline 6 & 6 & 12 & 12 & 12 & 12 & 12 & 12 & 12 & 12 & 16 & 12 \\
\hline 7 & 8 & 2 & 2 & 2 & 0 & 0 & 0 & 2 & 0 & 4 & 2 \\
\hline 8 & 10 & 10 & 14 & 14 & 14 & 10 & 10 & 10 & 14 & 14 & 14 \\
\hline 9 & 12 & 4 & 4 & 4 & -2 & -2 & -2 & 4 & -2 & 2 & 4 \\
\hline 10 & 14 & 8 & 16 & 16 & 16 & 8 & 8 & 8 & 16 & 12 & 16 \\
\hline 11 & 16 & 6 & 6 & 6 & -4 & -4 & -4 & 6 & -4 & 6 & 6 \\
\hline
\end{tabular}

It was noticed that the indicated 0 degree angle of attack had a non-zero lift coefficient, and thus the geometric angle of attack was determined relative to the wind tunnel. The geometric angle of attack for the baseline model was found by measuring the distance from the wind tunnel test section wall. Table 5.3 shows the correlation of the indicated angle of attack and the geometric angle of attack, the geometric angle of attack has been used in the discussion of results for this investigation.

Table 5.3: Correlation between indicated and geometric angles of attack for the baseline model

\begin{tabular}{|c|c|c|}
\hline Indicated AoA & Geometric AoA & Difference \\
\hline deg & deg & deg \\
\hline-4 & -2.39 & 1.61 \\
\hline-2 & -0.14 & 1.86 \\
\hline 0 & 2.49 & 2.49 \\
\hline 2 & 3.73 & 1.73 \\
\hline 4 & 5.27 & 1.27 \\
\hline 6 & 8.16 & 2.16 \\
\hline 8 & 9.70 & 1.70 \\
\hline 10 & 11.65 & 1.65 \\
\hline 12 & 13.70 & 1.70 \\
\hline 14 & 15.38 & 1.38 \\
\hline 16 & 17.47 & 1.47 \\
\hline
\end{tabular}

\subsubsection{Baseline Model Data Reduction}

The tab delimited data files collected during the wind tunnel testing were read into the data reduction programs shown in Appendix C. In the computer programs the average value of the forces (FA1, FA2, FB1, FB2) were determined in order to find the 
lift, L, and drag, D, forces using Equation 5.1, and Equation 5.2, based on the forces, F, measured by each load cell.

$$
\begin{aligned}
& L=F_{A_{1}}+F_{A_{2}} \\
& D=F_{B_{1}}+F_{B_{2}}
\end{aligned}
$$

The lift and drag forces were then converted to coefficients using the conventional definition of force coefficient, $\mathrm{C}_{\mathrm{F}}$, as defined by Equation 5.3. The force coefficient is dependent upon the free stream velocity, $\mathrm{V}$, the density of air, $\rho$, the model wing area, $\mathrm{A}_{\text {model }}$, and the measured force, $\mathrm{F}$.

$$
C_{F}=\frac{F}{1 / 2 \rho V^{2} A_{\text {model }}}
$$

\subsubsection{Error Analysis}

The measurement error is determined for any calculated term, $\mathrm{W}_{\mathrm{r}}$, based on the instrument errors, $\mathrm{w}_{\mathrm{i}}$, the partial derivatives of the resultant term, $\mathrm{R}$, with respect to each measured quantity, $x_{i}$, and the instrumentation uncertainty as shown in Equation 5.4. The primary calculated terms are the coefficients of lift, drag, and pressure, as seen in Equations 5.5 through 5.7. Typical errors for the lift and drag coefficient calculation were found to be 0.0016 and 0.0126 , respectively.

$$
\begin{gathered}
W_{r}=\left[\sum_{i=1}^{n}\left(\frac{\partial R}{\partial x_{i}} w_{i}\right)^{2}\right]^{1 / 2} \\
W_{C_{L}}=\left[\left(\frac{2 w_{L}}{\rho V_{\infty}^{2} A}\right)^{2}+\left(\frac{2 L w_{\rho}}{\rho^{2} V_{\infty}^{2} A}\right)^{2}+\left(\frac{4 L w_{V_{\infty}}}{\rho V_{\infty}^{3} A}\right)^{2}+\left(\frac{2 L w_{A}}{\rho V_{\infty}^{2} A^{2}}\right)^{2}\right]^{1 / 2} \\
W_{C_{D}}=\left[\left(\frac{2 w_{D}}{\rho V_{\infty}^{2} A}\right)^{2}+\left(\frac{2 D w_{\rho}}{\rho^{2} V_{\infty}^{2} A}\right)^{2}+\left(\frac{4 D w_{V_{\infty}}}{\rho V_{\infty}^{3} A}\right)^{2}+\left(\frac{2 D w_{A}}{\rho V_{\infty}^{2} A^{2}}\right)^{2}\right]^{1 / 2}
\end{gathered}
$$




$$
W_{C_{P}}=\left[\left(\frac{2 w_{P}}{\rho V_{\infty}^{2}}\right)^{2}+\left(\frac{2 w_{P_{\infty}}}{\rho V_{\infty}^{2}}\right)^{2}+\left(\frac{2\left(P-P_{\infty}\right) w_{\rho}}{\rho^{2} V_{\infty}^{2}}\right)^{2}+\left(\frac{4\left(P-P_{\infty}\right) w_{V_{\infty}}}{\rho V_{\infty}^{3}}\right)^{2}\right]^{1 / 2}
$$

However, the density, free stream velocity and wing area are also calculated values, thus their uncertainty needs to be determined. Equations 5.8 through 5.10 which show the derived uncertainty relationships for the density, $\rho$, free stream velocity, $\mathrm{V}_{\infty}$, and area, A, respectively are:

$$
\begin{array}{cc}
W_{\rho}=\left[\left(\frac{w_{P_{\infty}}}{R T}\right)^{2}+\left(\frac{P_{\infty} w_{T}}{R T^{2}}\right)^{2}\right]^{1 / 2}, & \text { Equation 5.8 } \\
W_{V_{\infty}}=\left[\left(\frac{w_{\Delta P}}{2} \sqrt{\frac{2}{\rho(\Delta P)}}\right)^{2}+\left(\frac{w_{\rho}}{2} \sqrt{\frac{2(\Delta P)}{\rho^{3}}}\right)^{2}\right]^{1 / 2}, & \text { Equation 5.9 } \\
W_{A}=\left[\left(b w_{c}\right)^{2}+\left(c w_{b}\right)^{2}\right]^{1 / 2} . & \text { Equation 5.10 }
\end{array}
$$

Since the errors are dependent upon the values discussed in the results, the values of the errors are presented with the results sections for the baseline model in Section 5.6.3.

\subsubsection{Baseline Airfoil Results}

Experimental testing of the baseline model revealed the lift curve for the elliptical profile with a rounded trailing edge, used for comparison to the circulation controlled model in determining the representative change in angle of attack. Figure 5.28 compares the results of the lift curve for a NACA 0010-34 airfoil [Abbott and vonDoenhoff, 1959] to the $\mathrm{CCHB}$ lift curve. The error bars in Figure 5.29 represent the average instrumentation error of the 11 test cases, calculated using Equations 5.5 - 5.10. The rounded trailing edge of the CCHB model slightly alters the lift curve from that of the NACA 0010-34 airfoil at moderate angles of attack. For small angles of attack $(<5$ degrees) the CCHB lift curve closely resembles the NACA airfoils lift performance. 
Thus, the rounded trailing edge alters the lift performance of an airfoil, even with no active blowing slot, at angles of attack greater than 5 degrees.

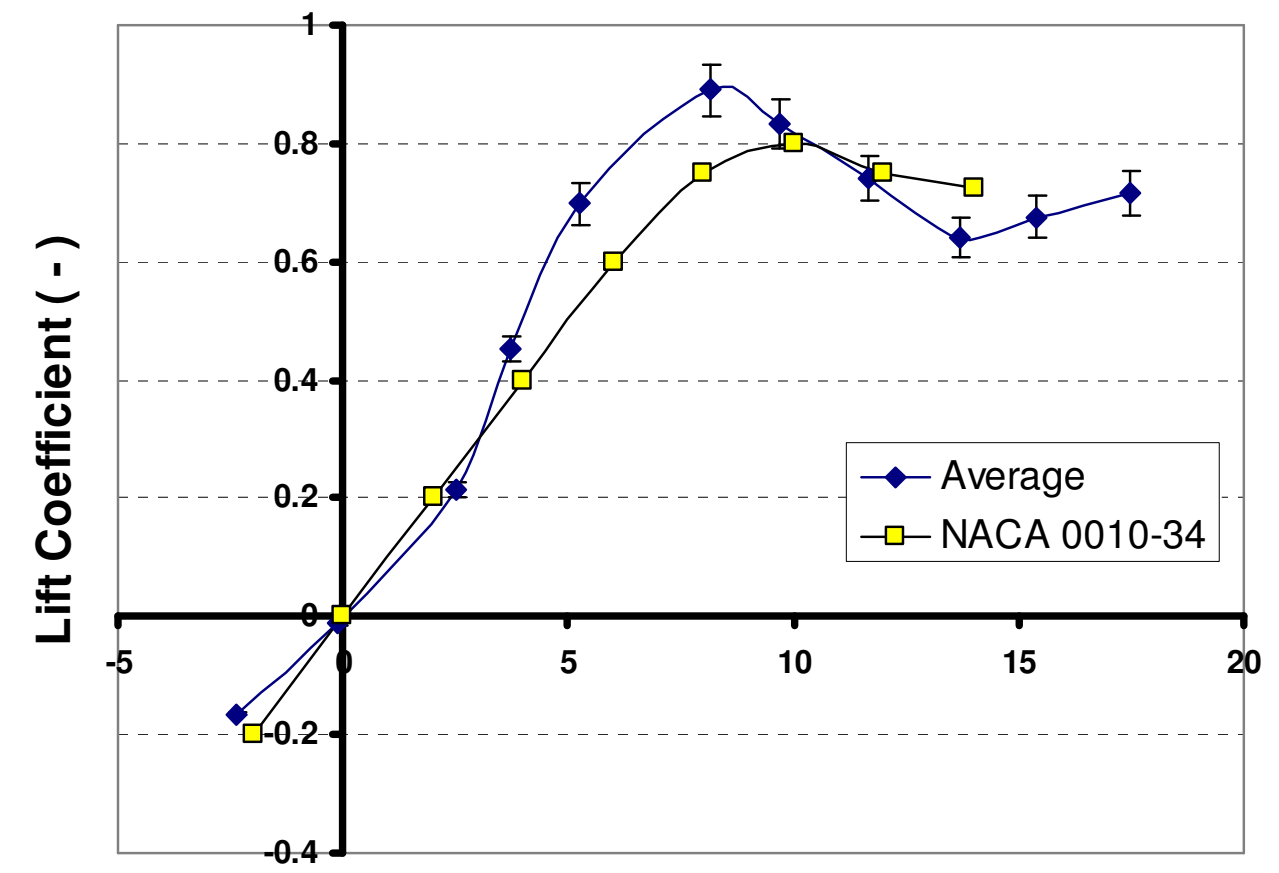

Angle of Attack (deg)

Figure 5.28: Baseline test results compared to the NACA 0010-34 airfoil

Figure 5.29 shows the comparison of the Abbot and vonDoenhoff [1959] NACA 0010-34 drag coefficients to the measured drag for the baseline CCHB model. The relatively high computed uncertainties in the drag values are primarily due to the smaller force magnitudes as compared to the lift coefficient. The trailing edge geometry also influences the drag force, as expected, due to the rounded trailing edge. The CCHB trailing edge varies the location of the separation point, due to the relaxation of the Kutta condition at the trailing edge. The flow separation point of the NACA 0010-34 is at the trailing edge of the airfoil as defined by the Kutta condition as the sharp trailing edge, unless the airfoil stalls at high angles of attack. However, the drag coefficient values are reasonably close in the range of lift coefficients between -0.2 to +0.4 . 


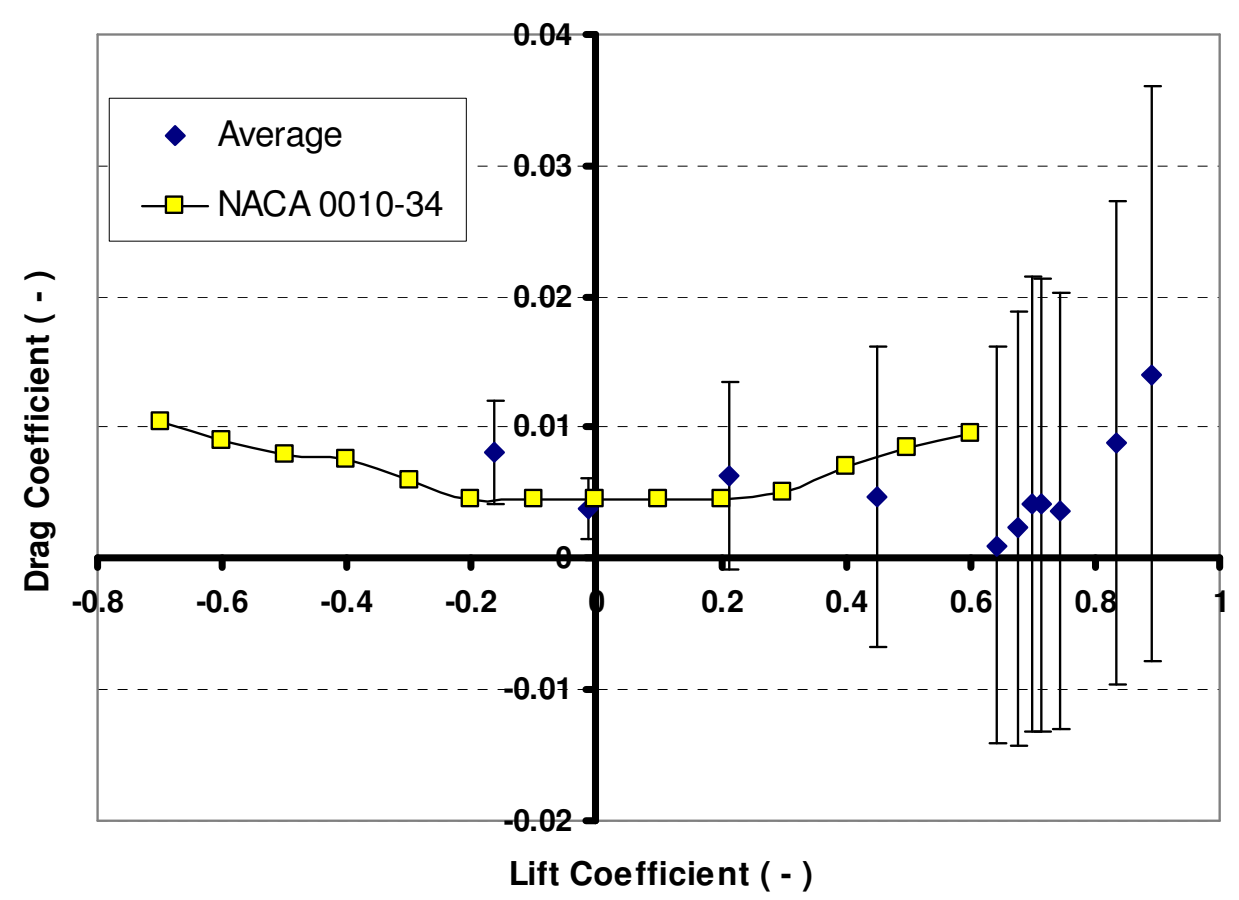

Figure 5.29: Drag coefficient measured for the 10:1 elliptic airfoil compared to the NACA 0010-34 drag polar

\subsection{Active Circulation Control Model Experimental Testing}

The circulation control model testing was primarily done at 0 degree angle of attack, with several tests being conducted at $-2,2$ and 6 degrees. The small angles of attack were used to minimize the influence of the tunnel walls on the flow field around the model, particularly in the wake of the model with circulation control. The source of the circulation control air was a bleed system from the WVU Blowdown Wind Tunnel, which was controlled by two pressure regulators placed in the system, one to control the pressure of the leading edge and one for the trailing edge.

Thus, the resulting jet exit velocity was determined based on a calibration curve, versus the regulated pressure, resulting in Figure 5.30. Once the jet velocity was known, the blowing coefficient was determined for comparison purposes. In this testing program, the primary regulated pressures used were $10 \mathrm{psig}, 20 \mathrm{psig}$ and $30 \mathrm{psig}$. Several tests were run at higher pressures ( 40 and 60 psig) to verify that the maximum jet velocity (choked flow) was achieved. 


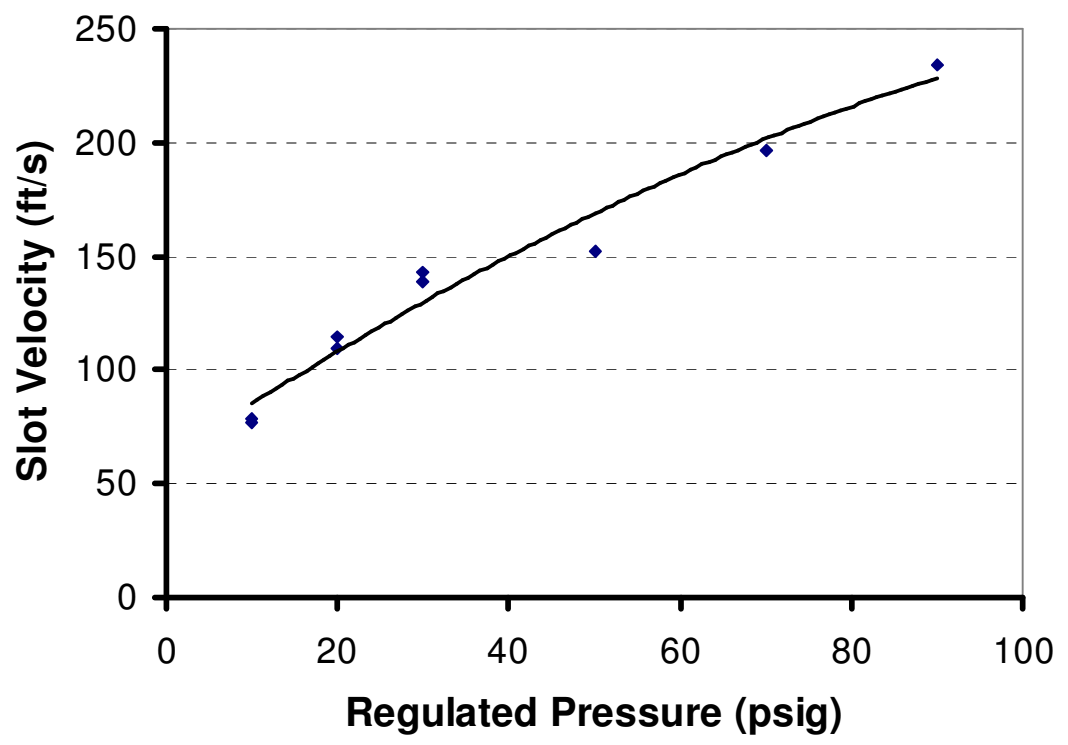

Figure 5.30: Jet exit velocity as measured based on the regulated pressure

\subsubsection{Active Model Testing Procedure}

Since the circulation control air supply tubing could alter the force readings, the forces created by pressurizing the supply lines were recorded with combinations of leading and trailing edge pressures (regulated at 10,20 and 30 psig) at the wind speeds of approximately 50, 70, and $100 \mathrm{ft} / \mathrm{s}(0.6,1$, and 2 inches of water, respectively). These forces were removed from the steady state blowing tests as tare values with matching pressure conditions, using data such as shown in Appendix D.

Two test matrices were used to analyze the two primary aspects: magnitude of augmentation for a $10 \%$ chord thick airfoil, and the time required to establish the force augmentation, with the same test model. To determine the force augmentation ratio $\left(\Delta \mathrm{C}_{1} / \mathrm{C}_{\mu}\right)$ the test matrix shown in Table 5.4 was used with steady blowing at the prescribed settings. Through the use of solenoids to activate the rotary valves in the model, dynamic tests were performed with combinations of conditions outlined in Table 5.5. The movement of the solenoids created a force that was recorded by the measurement system, as previously discussed in Section 5.4, which were later removed as tare values. To ensure consistent starting times of the solenoid movement the data acquisition program was used to control the valve actuation with a start time $50 \mathrm{msec}$ after the start of data collection. 
Table 5.4: Steady state testing conditions used for force augmentation study

\begin{tabular}{|c|c|c|c|}
\hline $\begin{array}{c}\text { Angle of Attack } \\
(\mathrm{deg})\end{array}$ & $\begin{array}{c}\text { Wind Speed } \\
\text { (approximate ft/s) }\end{array}$ & $\begin{array}{c}\text { Leading Edge } \\
\text { Regulated Pressure } \\
(\mathrm{psig})\end{array}$ & $\begin{array}{c}\text { Trailing Edge } \\
\text { Regulated Pressure } \\
(\mathrm{psig})\end{array}$ \\
\hline \multirow{4}{*}{0} & $50,70,100$ & 0 & $0,10,20,30$ \\
\cline { 2 - 4 } & $50,70,100$ & 10 & $0,10,20,30$ \\
\cline { 2 - 4 } & $50,70,100$ & 20 & $0,10,20,30$ \\
\cline { 2 - 4 } & $50,70,100$ & 30 & $0,10,20,30$ \\
\hline \multirow{3}{*}{2} & $50,70,100$ & 10 & $0,10,20,30$ \\
\cline { 2 - 4 } & $50,70,100$ & 20 & $0,10,20,30$ \\
\cline { 2 - 4 } & $50,70,100$ & 30 & $0,10,20,30$ \\
\hline
\end{tabular}

Table 5.5: Test parameters for the dynamic tests

\begin{tabular}{|c|c|c|c|c|}
\hline $\begin{array}{c}\text { Angle of } \\
\text { Attack } \\
(\mathrm{deg})\end{array}$ & $\begin{array}{c}\text { Approximate } \\
\text { Wind Speed } \\
(\mathrm{ft} / \mathrm{s})\end{array}$ & $\begin{array}{c}\text { Leading Edge } \\
\text { Regulated } \\
\text { Pressure }(\mathrm{psig})\end{array}$ & $\begin{array}{c}\text { Trailing Edge } \\
\text { Regulated Pressure } \\
(\mathrm{psig})\end{array}$ & Valve Direction \\
\hline$-2,0,2,6$ & $50,70,100$ & $0,10,20,30$ & $0,10,20,30$ & Opening/closing \\
\hline 2 & 100 & $0,10,20$ & 40 & Opening/closing \\
\hline
\end{tabular}

\subsubsection{Active Model Experimental Results}

The same load cell configuration was used in the testing of both the baseline and active models. The results of the active model testing were compared to historical data from Kind and Maull [1968] and the baseline model. Three primary blowing pressures were used in the experimental analysis with additional blowing coefficients achieved by reducing the freestream velocity.

\subsubsection{Aerodynamic Force Coefficient Comparisons}

To verify the similarity of the two circulation control elliptical models, geometric angles of attack of $-2,0,2$, and 6 degrees were tested with the Active model without circulation control blowing and compared to the lift curve of the Baseline model. Figure 5.31 compares the lift curve slope of the Active Circulation Control Model to the 
Baseline model lift curve. From this figure it is seen that lift curve slope for the Active model tracks and is slightly higher (2\%) than the Baseline model, thus the aerodynamic performance of the two models will be approximately the same. There are several sources of error that are expected to contribute to the difference in lift performance, which are discussed in Section 5.7.3.

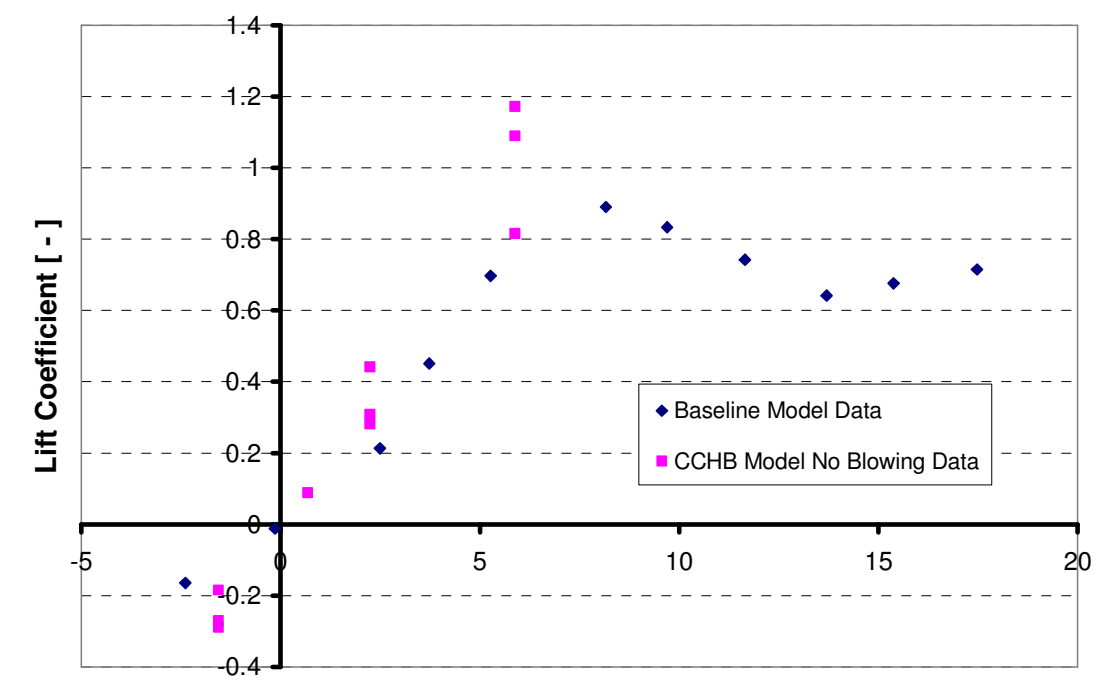

Angle of Attack [deg]

Figure 5.31: Lift curve comparison of the baseline and active models

Comparisons of the results to historical data from Kind and Maull [1968], one of the few prior investigations with a $10 \%$ chordlength thick airfoil, are shown in Figure 5.32. From this figure it was determined that the steady state performance of the elliptical airfoil was $50 \%$ different, at a blowing coefficient of 0.03 , than that found by Kind and Maull [1968]. The general trend, increasing lift coefficient as the blowing coefficient is increased is observed. Possible causes for the discrepancies between the historical data and the data collected with the trailing edge blowing are reconciled in the error analysis sub-section 5.7.3. With two of the most likely being the fact that the present model effectively had no blowing over the top $20 \%$ of the span and a discontinuity in the trailing edge curvature. 


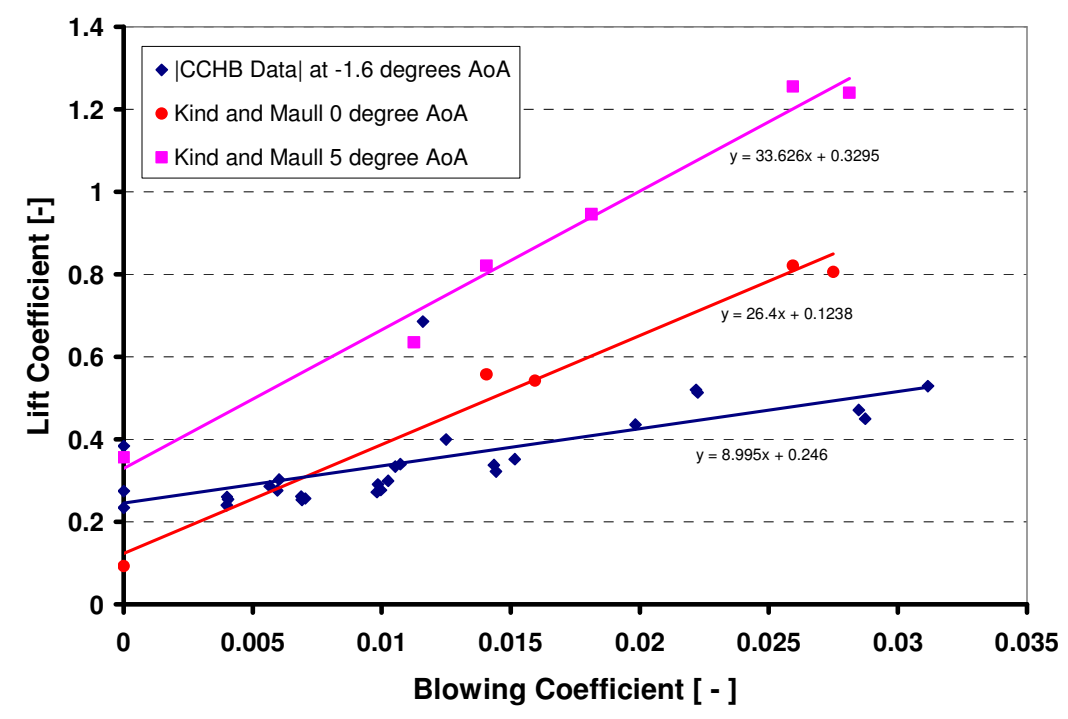

Figure 5.32: Lift coefficient as dependent upon the blowing coefficient data at a Reynolds number of $4 \times 10^{6}$, compared to the results from Kind and Maull [1968].

The lift coefficient augmentation created by the leading edge blowing slot was found to have a greater influence in the lift force relative to trailing edge blowing, as shown in Figure 5.33. This increase is primarily artificial in nature due to the reaction force to the jet being in the lift direction. A similar reduction in drag was found for the trailing edge blowing slots, Figure 5.34, where the momentum of the jet creates a small thrust component.

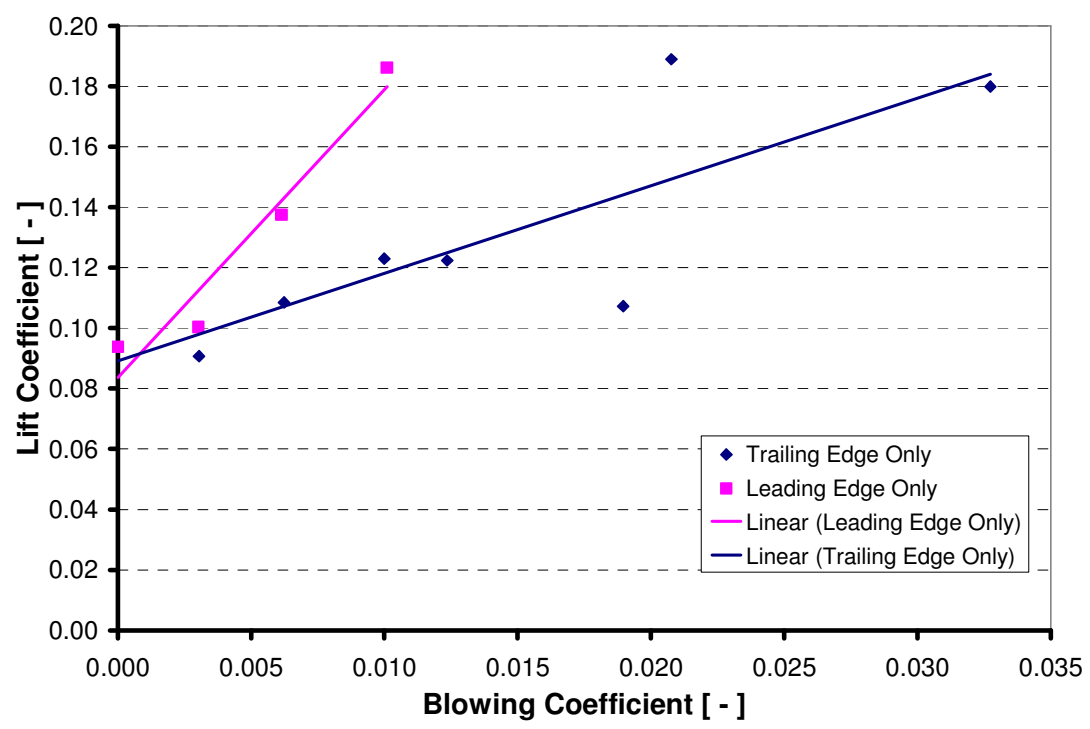

Figure 5.33: Lift Coefficients for the CCHB Active Model at 0.68 degrees angle of attack with one slot blowing at a Reynolds number of $4 \times 10^{6}$. 


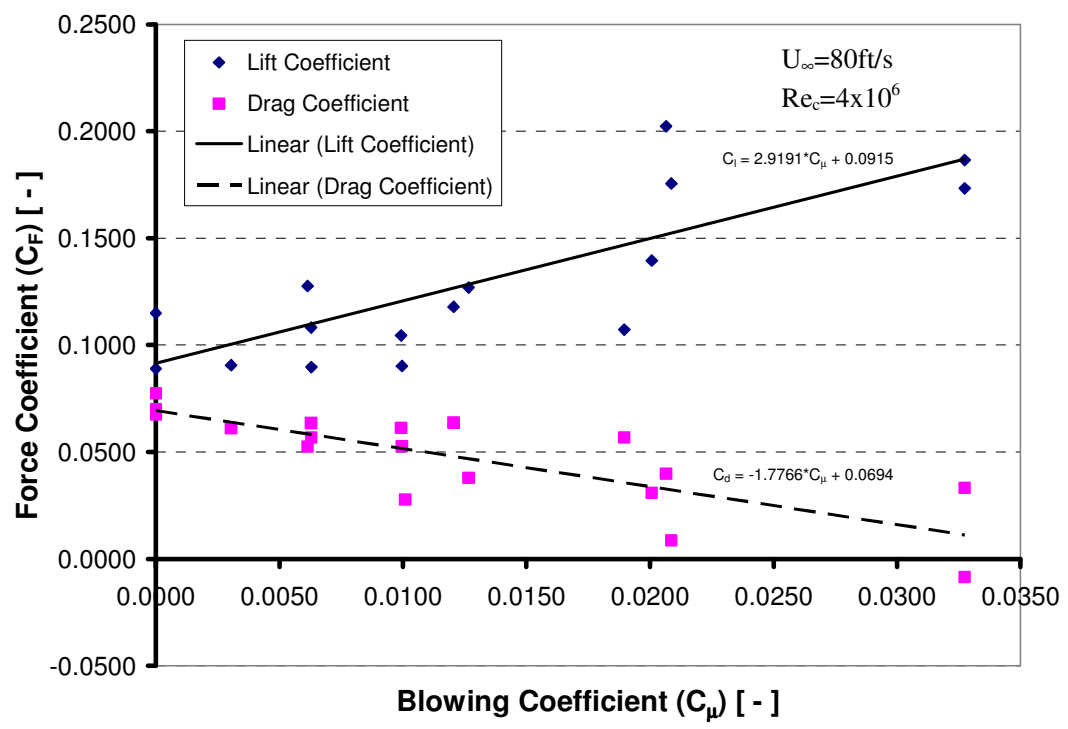

Figure 5.34: Lift and drag force coefficients measured for various blowing coefficients for the CCHB active model at 0.68 degrees angle of attack

Combinations of the leading and trailing edge blowing conditions were also tested. Figure 5.35 shows the results for the test cases where the leading edge blowing slot was set at $10 \mathrm{psig}$, where the different leading edge blowing coefficients are created by changing the wind tunnel airspeed, thus the freestream momentum. The data indicates that for the conditions tested, the leading edge interferes with the lift augmentation performance of the trailing edge blowing slot as the blowing coefficient is increased. As the regulated pressure is increased, Figure 5.36 for 20 psig and Figure 5.37 for 30 psig, the interference between the blowing slots was increased. The tabulated data for these cases are shown in Appendix E. In each of these three figures, it was found that for the leading edge blowing slot configuration tested in this investigation, increases in the leading edge blowing coefficient, by reducing the wind tunnel velocity, increased the lift coefficient and altered the effectiveness of the trailing edge, Coanda jet. It was also found that increasing the leading edge regulated pressure reduced the lift coefficient for equivalent blowing coefficients. 


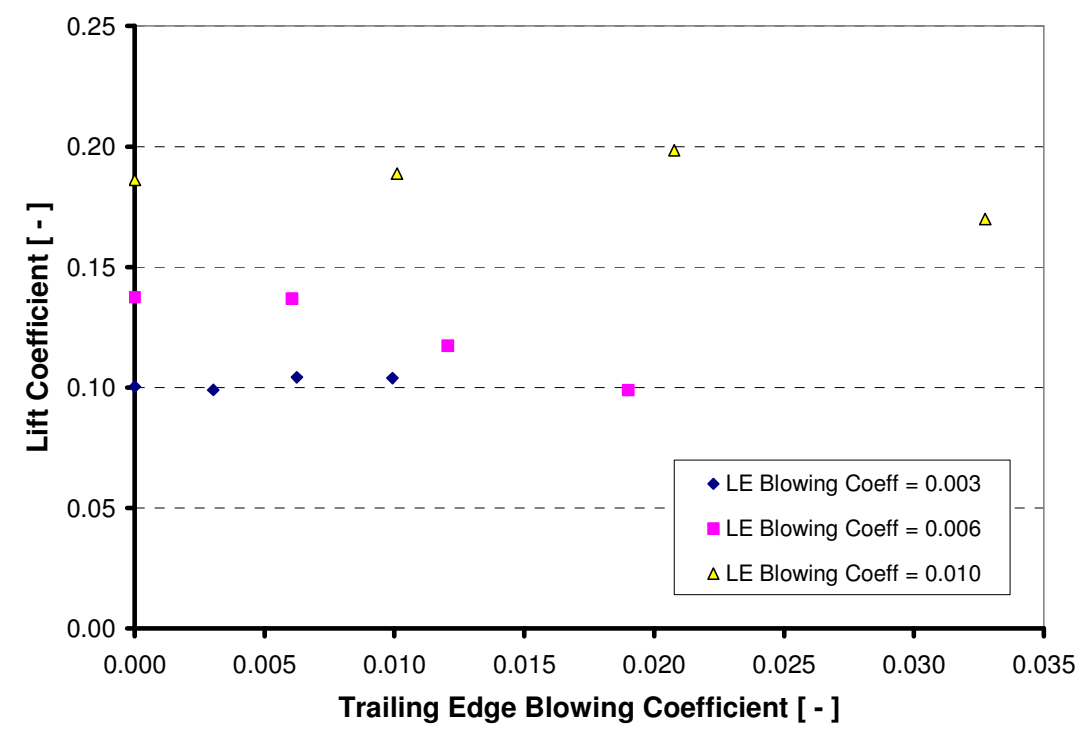

Figure 5.35: Lift coefficients for the CCHB active model at 0.68 degrees angle of attack with a leading edge pressure of 10 psig

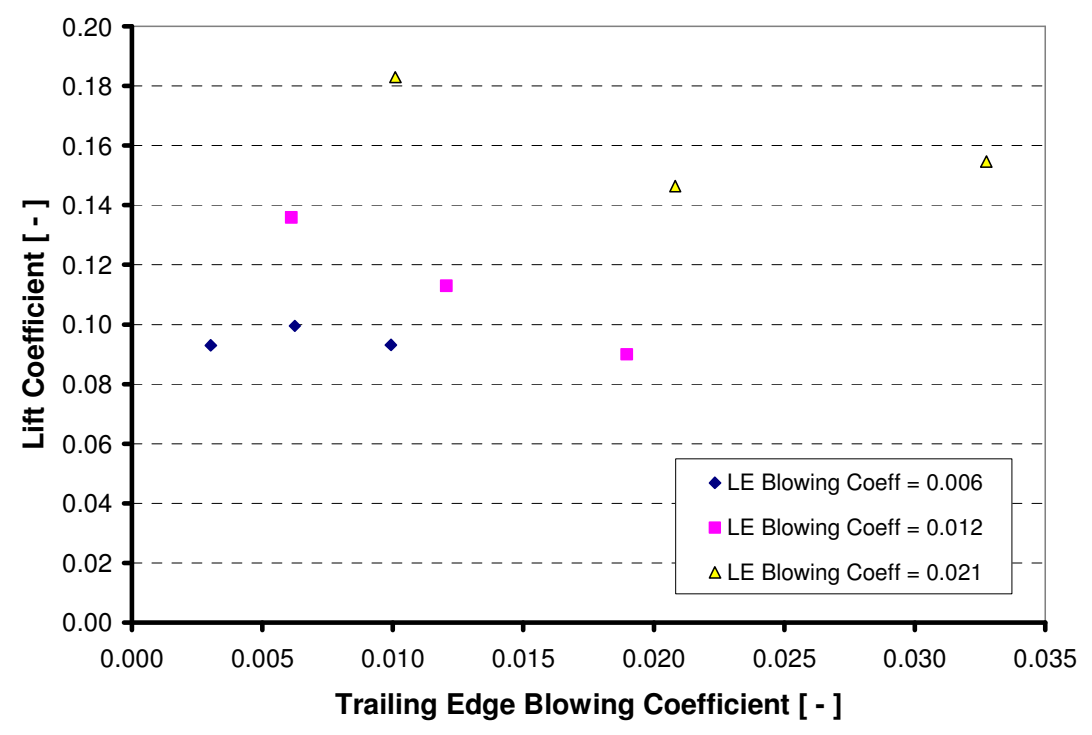

Figure 5.36: Lift coefficients for the CCHB active model at 0.68 degrees angle of attack with a leading edge pressure of 20 psig 


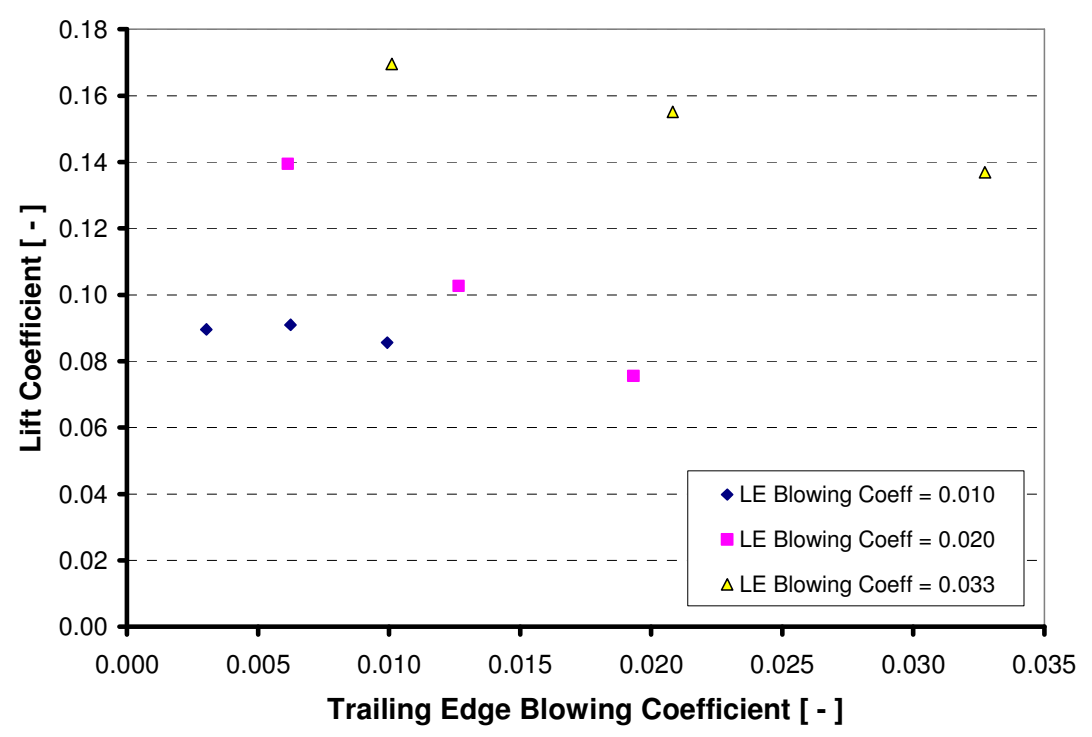

Figure 5.37: Lift coefficients for the CCHB active model at 0.68 degrees angle of attack with a leading edge pressure of $30 \mathrm{psig}$

This part of the analysis also showed that the leading edge blowing slot was more effective than the trailing edge blowing slot. However, a combined blowing case produced a larger lift coefficient than either the leading or trailing edge alone, but also at a higher mass flow rate. Thus the most effective blowing condition tested was with the leading edge regulated pressure of $10 \mathrm{psig}$, and a LE blowing coefficient of 0.003 and the trailing edge blowing coefficient of 0.003 , resulting in a lift coefficient of 0.1 . This indicates that a trade-off needs to be made between the power/mass flow requirements and the circulation control performance.

\subsubsection{Reaction Time Comparisons}

The second objective of this research was to determine the reaction time of the forces created by the freestream airflow to the introduction of the blowing jet. In order to determine these, it is necessary to know the time response characteristics of the components controlling the opening of the rotary valve. The system that actuated the valve consisted of a relay, which has a 10 millisecond response time to energize, and a solenoid. The time for the solenoid to move was measured for opening and closing of the valve as 46 and 45 milliseconds, respectively, and the activation response is shown in Figure 5.38 with 0 seconds indicating the initiation of the solenoid activation from the 
computer and the "jump" in voltage at 0.056 seconds indicating the solenoid has completed its movement. With the system response known the aerodynamic response times could be estimated.

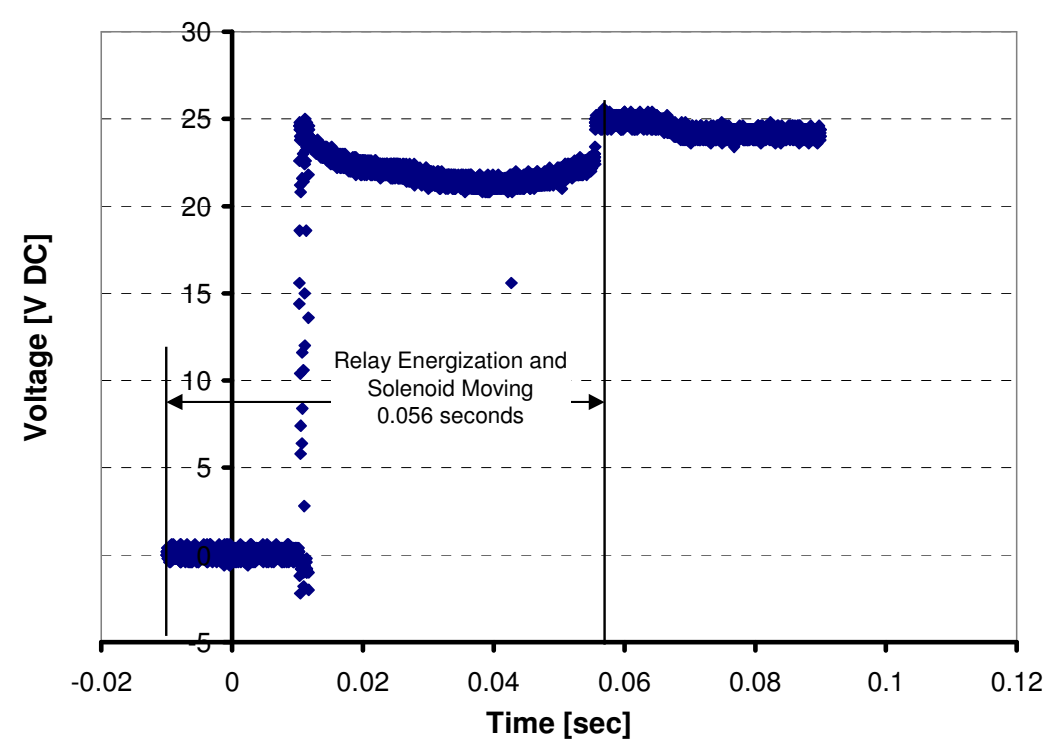

Figure 5.38: Solenoid opening time requirement measurement

Figure 5.39 shows the recorded lift force for the case with the trailing edge blowing slot closing while regulated at 30 psig and at a 0.68 degree angle of attack. In this data file a 0.050 second delay was set for the initiation of the solenoid, after incorporating the 65 seconds for the relay and solenoid the blowing slot was fully opened at 0.105 seconds. Though there is a larger amplitude to the recorded force after the opening of the slot, the mean of this cyclic force is stabilized shortly (within 5 milliseconds) after the valve is opened by the solenoid. The response of the drag force is shown in Figure 5.40. 


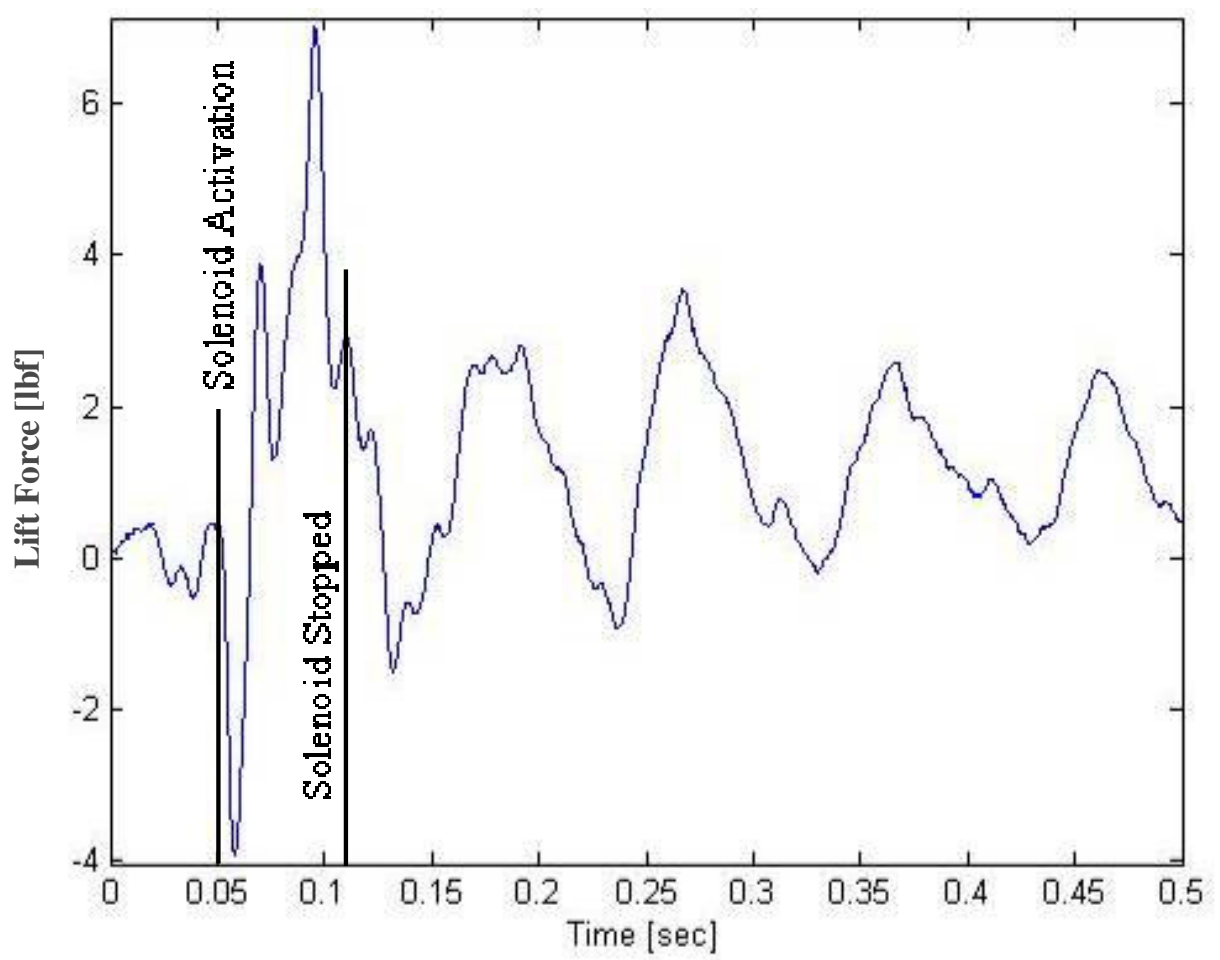

Figure 5.39: Experimental time response for lift with the 30 psig regulated trailing edge closing test condtion at 0.7 degrees angle of attack

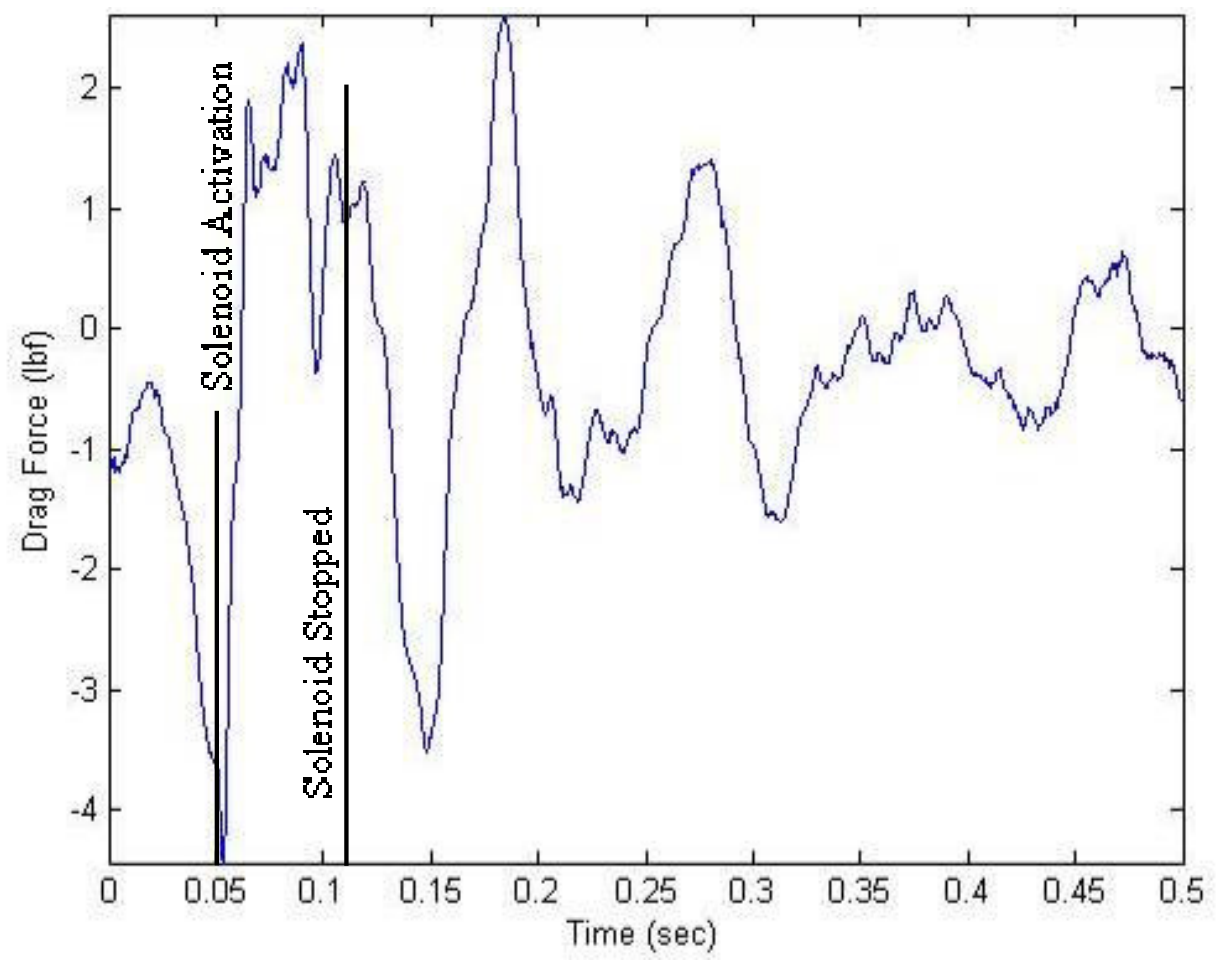

Figure 5.40: Experimental time response for drag with the 30 psig regulated trailing edge closing test condition at 0.7 degrees angle of attack 
The time response analysis revealed that the mean augmented force has occurred within the opening of the blowing slot. Little variation was found in the force stabilization time for the various blowing conditions.

\subsubsection{Error Analysis}

The relationships for the measurement errors are the same as that which was determined for the Baseline model with the addition of the error in the blowing coefficient, $\mathrm{C}_{\mu}$, as defined in Equation 5.11. From this relationship the typical error in blowing coefficient was found to be 0.03 .

$W_{C_{\mu}}=\left[\left(\frac{2 V_{j e t} w_{\dot{m}_{j e t}}}{\rho V_{\infty}^{2} A}\right)^{2}+\left(\frac{2 \dot{m}_{j e t} w_{V_{j e t}}}{\rho V_{\infty}^{2} A}\right)^{2}+\left(\frac{2 \dot{m}_{j e t} V_{j e t} w_{\rho}}{\rho^{2} V_{\infty}^{2} A}\right)^{2}+\left(\frac{4 \dot{m}_{j e t} V_{j e t} w_{V_{\infty}}}{\rho V_{\infty}^{3} A}\right)^{2}+\left(\frac{2 \dot{m}_{j e t} V_{j e t} w_{A}}{\rho V_{\infty}^{2} A^{2}}\right)^{2}\right]^{1 / 2}$

Equation 5.11

Potential sources of error in the experimental testing of the active model include, but are not limited to, the slight modification to the shape by the blowing slots and associated seals. The rubber seals separating the leading edge plenum from the trailing edge plenum added approximately 0.04 inches $(0.3 \% \mathrm{c})$ to the thickness of the airfoil at $50.8 \%$ of the chordlength. Another source of error is the limited seals on the slots themselves. To prevent interference with the activation of the blowing slot, the seal used was just the contact between the models surfaces (upper and lower) and the rotating valve. Figure 5.41 shows the trailing edge of the Active model with the rotating valve in the closed position and Figure 5.42 is in the open position. 


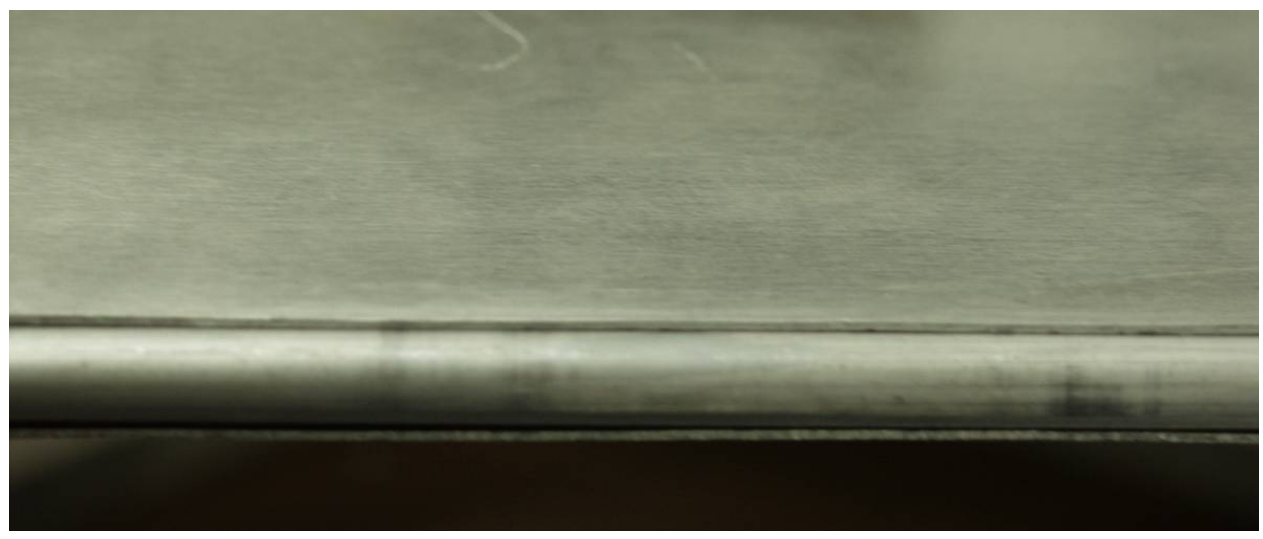

Figure 5.41: Trailing edge of the active model in the closed position

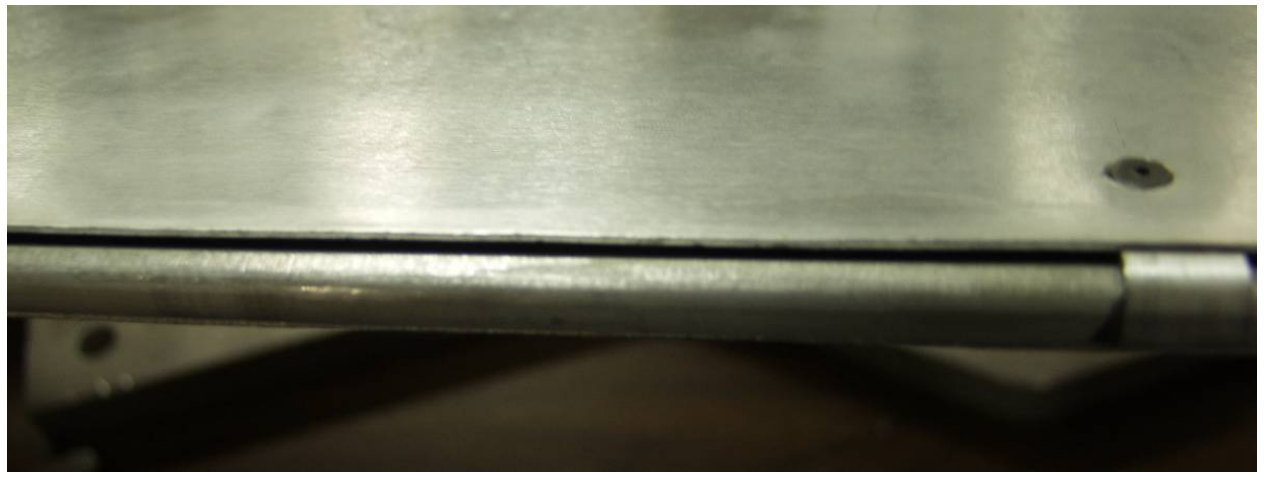

Figure 5.42: Trailing edge of the active model in the open position

A third source of error is the transition in radius from the open dimension to the closed value. Due to the size of the component (0.4 inches diameter and 36 inches in length) manufacture of a smooth contour between radii with available resources was difficult, thus there was a discontinuity in the radius which prematurely separates the circulation control jet from the Coanda surface, as discussed by Wood [1986]. The premature separation of the Coanda jet, due to the discontinuous surface, is expected to be responsible for the limited force augmentation and the disagreement with the data presented in Kind and Maull [1968].

A source of error that only impacted the time response data was the dynamic response of the load cells to the movement of the solenoids. The relatively large mass of the solenoids created a force that damped out of the reading in 2 seconds. Multiple measurements of the force response showed that the magnitude of the forces were within 0.4 pounds and thus were tared out of the data files. 


\section{Computational Investigation}

Another key parameter to helicopter performance is the blade azimuth angle, defined as the location of the blade within the rotor disc, typically defined from the nose of the aircraft and in the direction of rotation. The resultant velocity of the airflow over the blade affects the forces, by affecting the relative velocity magnitude and direction with respect to the blade, as seen in Figure 6.1 and Equation 6.1 below. The position of the blade relative to the direction of flight, or azimuth angle $(\psi)$, influences the lift generation. The lift force is also dependent upon the rotational speed of the blade, $\mathrm{V}_{\omega}$, which is a function of the radial position, $r$, and the rotational speed, $\omega$, of the rotor. The forward flight speed, $\mathrm{V}_{\infty}$, trigonometrically influences the creation of lift based on the azimuth angle of the blade. Thus the relative velocity, $\mathrm{V}_{\text {relative, }}$ is dependent upon the rotor speed, the radial position along the blade, the forward flight speed, the azimuth position, and the wind speed, $\mathrm{V}_{\text {wind }}$, and direction, $\gamma$. Figure 6.1 illustrates the parameters used in defining the relative velocity. The velocity magnitude of the flow over the rotor blade alters the magnitude of the force being created and is thus integral to the understanding of the required augmentation to the force coefficient needed to maintain stable flight of a helicopter.

$$
V_{\text {relative }}=\omega r+V_{\infty} \sin (\psi)+V_{\text {wind }} \sin (\psi-\gamma) \quad \text { Equation } 6.1
$$

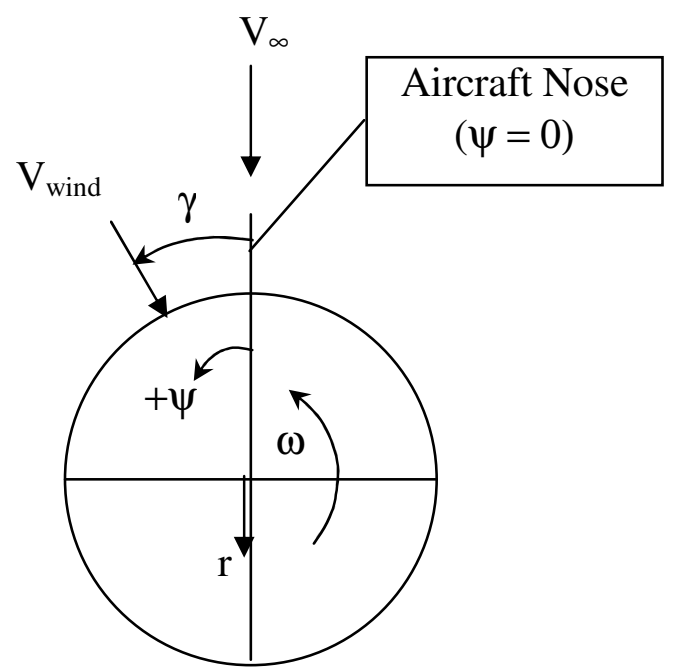

Figure 6.1: Representation of azimuth location 
To enhance this feasibility study a computational investigation into the influence of the orientation (azimuth angle) of the rotor blade with respect to the free stream velocity on the aerodynamic performance was conducted by adjusting the jet and freestream velocities, in unsteady two-dimensional modeling, as well as limited threedimensional cases. This study will be used in the prediction of the blades lift profile and necessary scheduling of the opening and closing of the circulation control blowing slots using the commercial Fluent CFD software package.

\subsection{Computational Geometry}

The two-dimensional computational grids used in this investigation were built in Gambit, the commercially available preprocessing software for the Fluent computational flow solver, based on the experimental setup. The two-dimensional computational grid was primarily used to determine predictions of both the reaction time and the force augmentation. A three dimensional grid was setup to estimate the influence of sweep on the circulation control effectiveness.

\subsubsection{Two-Dimensional Grid Setup}

A two-dimensional cross-section of the experimental test section was modeled computationally, as shown in Figure 6.2. The leading and trailing edge blowing slots were divided into three intervals, with 1693 node points evenly dispersed around the remainder of the model. A ratio of 1.05 was used to stretch the mesh away from the blowing slot, as shown in Figure 6.3 for the trailing edge and Figure 6.4 for the leading edge. The four blowing slots were all set as velocity inlets with the velocities ranging from 400 to 1600 feet per second to vary the blowing coefficient. The remaining surfaces

of the airfoil model were defined as walls, with a no slip boundary condition, as were the upper and lower surfaces of the grid. A velocity inlet, to the left in Figure 6.2, was used as the uniform inlet to the flow field, and the right wall was defined as a pressure outlet. 


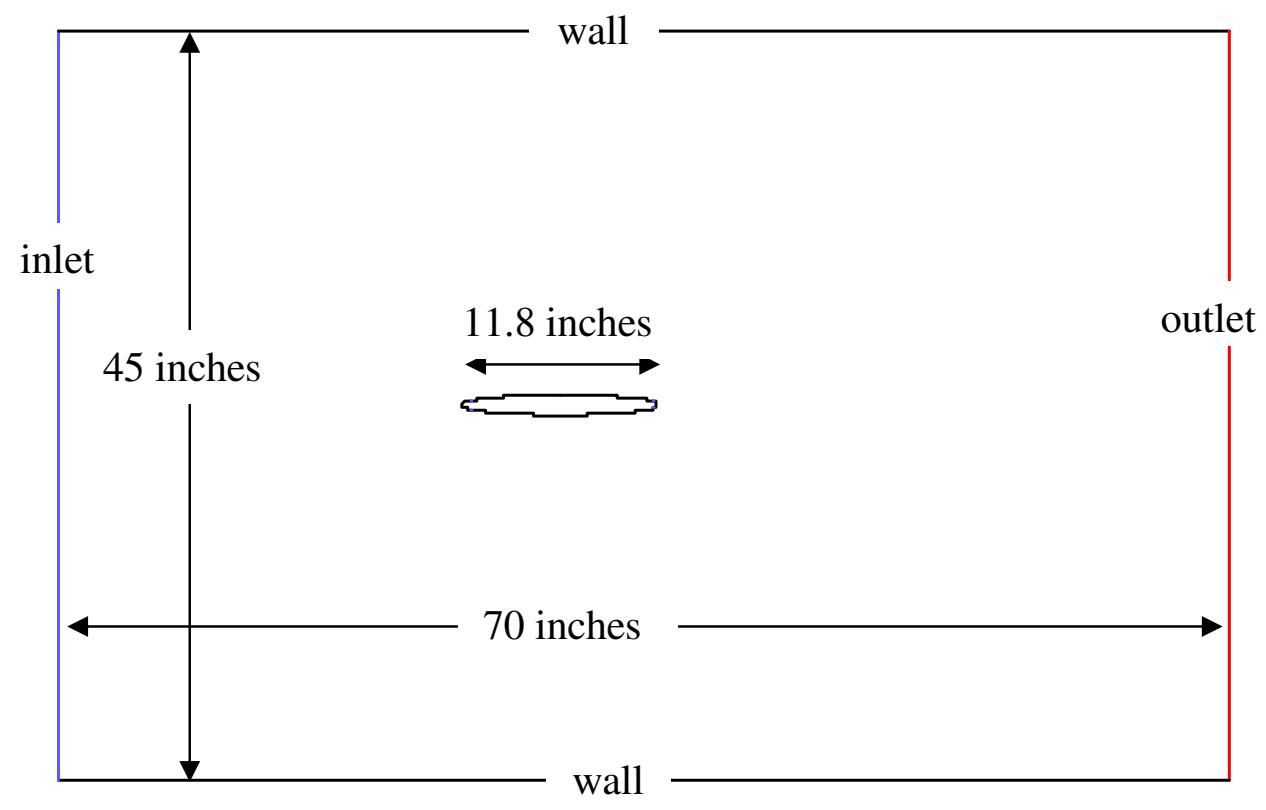

Figure 6.2: Computational space modeled for the two-dimensional study

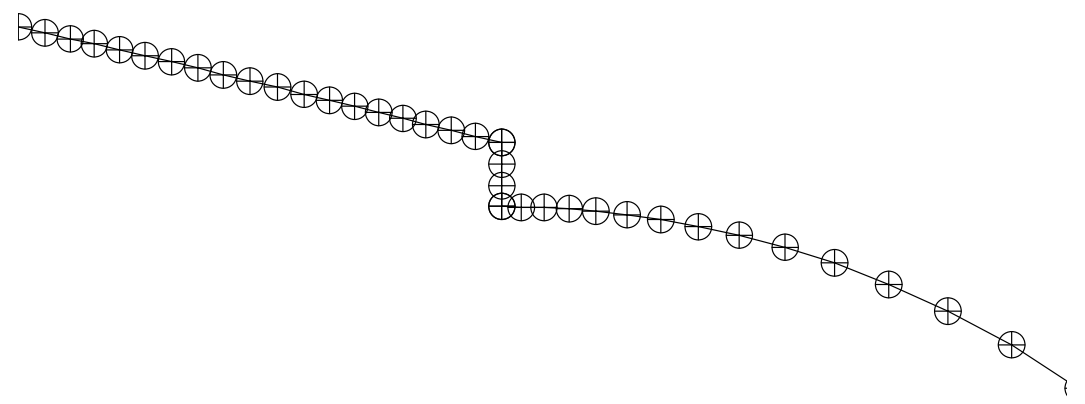

Figure 6.3: Computational node points on and near the upper surface, trailing edge blowing slot 


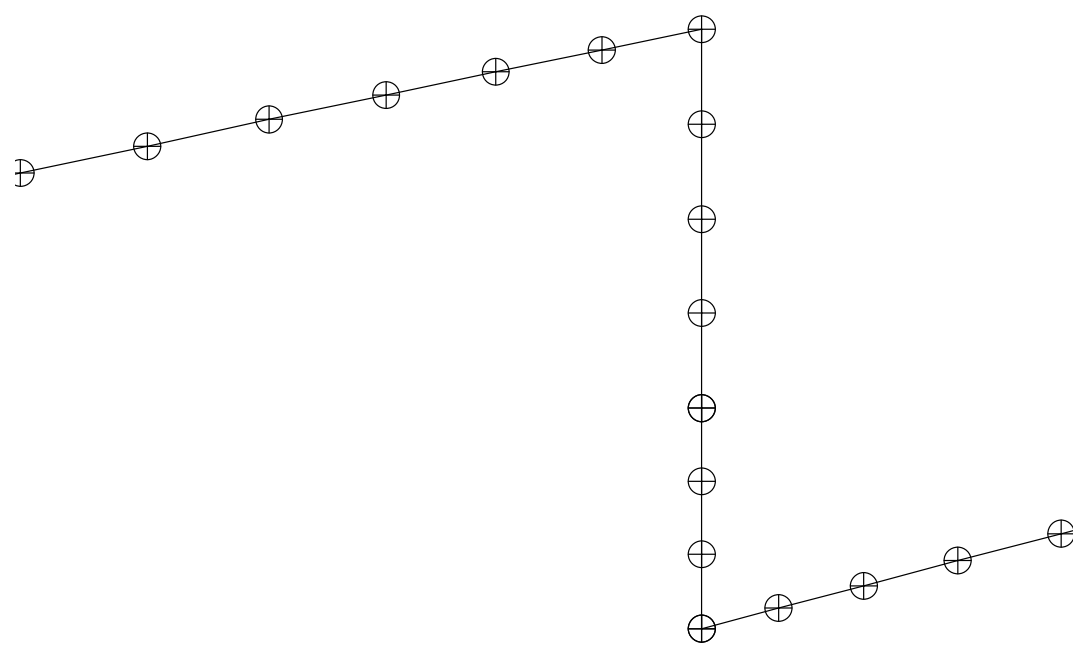

Figure 6.4: Computational grid point locations on, and near, the upper surface, leading edge blowing slot

A growth rate of 1.5 was used to expand the airfoil surface mesh to the twodimensional face defining the flow field. The maximum ratio of the sizes of two neighboring sides of the triangular mesh was set to be 1.5 inches. Figure 6.5 and Figure 6.6 show locally enlarged views of the mesh near the upper surface trailing and leading edges, respectively. An overview of the grids for the entire flow field is shown in Figure 6.7 and additional views of the computational matrix are shown in Appendix $\mathrm{H}$.

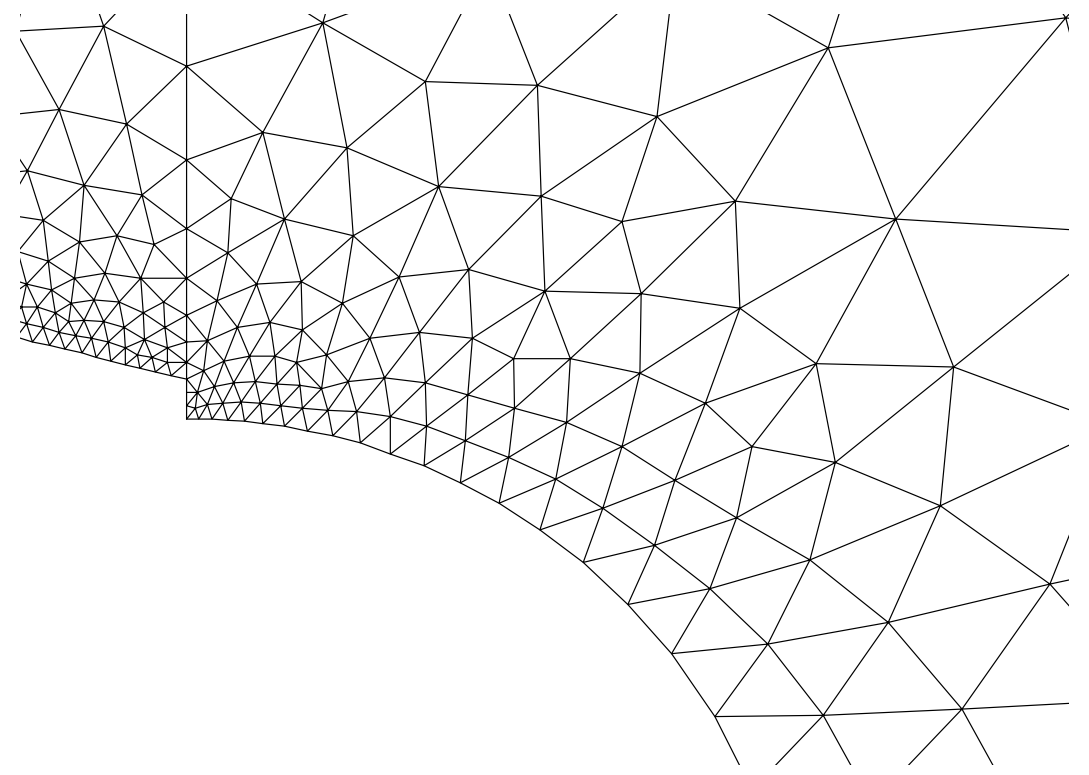

Figure 6.5: Locally enlarged view of the grid for the upper surface trailing edge blowing slot 


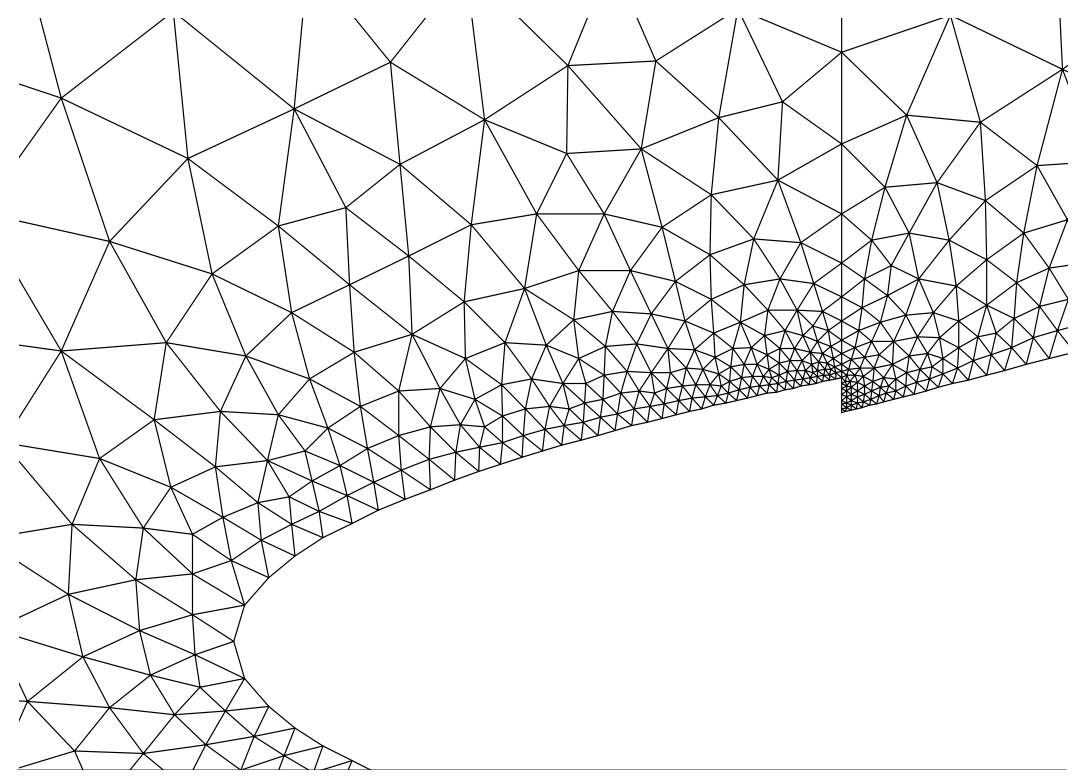

Figure 6.6: Locally enlarged view of the 2-D grid for the upper surface leading edge blowing slot

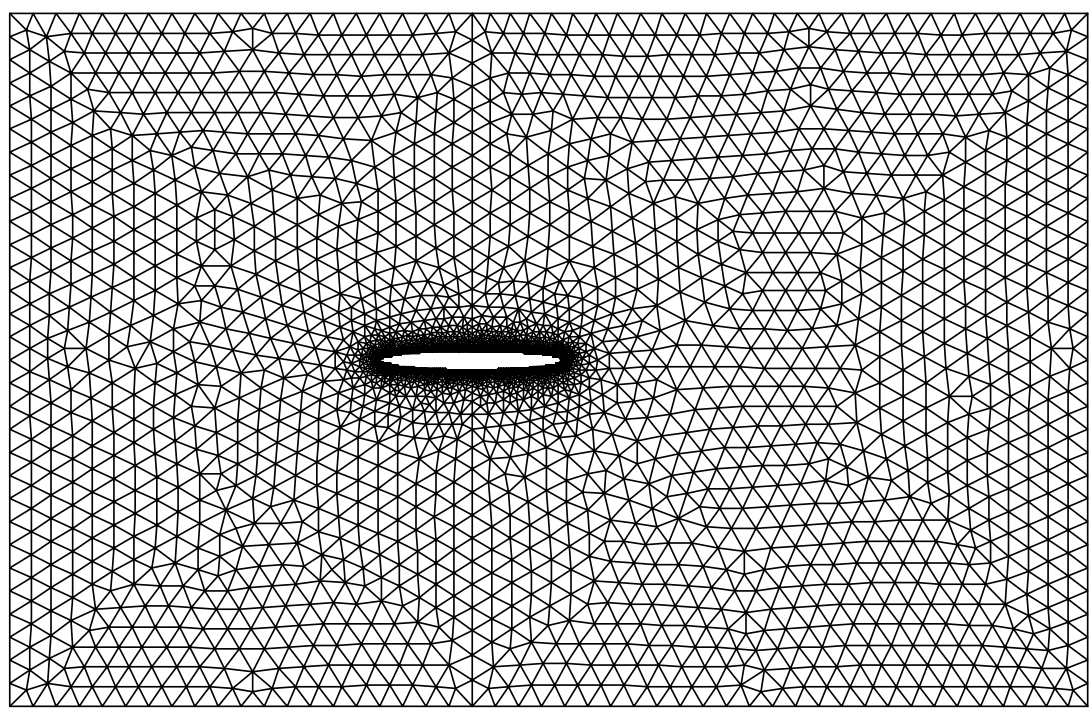

Figure 6.7: Overview of entire two dimensional grid

The two-dimensional computation grid contained 33,700 faces, and allowed for efficient use of computational time by not over-refining the mesh. By concentrating the grid near the airfoil, particularly near the blowing slots, the flow physics were modeled with manageable computational requirements and each case was able to be solved in two 
hours or less. The node spacing near the blowing slots was set to be 0.0025 inches, and the maximum sized element on the model, located near the mid-chord region, has a node spacing of 0.025 inches, considerably smaller than the grid independence as indicated in Section 6.3. A slightly higher growth rate was used to restrict the total number of computational node points.

\subsubsection{Three Dimensional Grid Setup}

A three-dimensional computational grid was developed to investigate the influence of flow angularity on the performance of the circulation control blowing. Two models were developed for this analysis, the first with the wing normal to the airflow as shown in Figure 6.8. The length of the geometry was 70 inches and the height was set to 45 inches, as in the two-dimensional study. The span of the computational space was set to 32 inches and the corners of the space were chamfered to resemble the physical space of the wind tunnel test section. The dimensions of the circulation control helicopter blade were the duplicated from the experimental and two-dimensional models, an elliptic airfoil with a chord length of 11.8 inches, and a thickness of 1.2 inches, and spanned the entire height of the computational space.

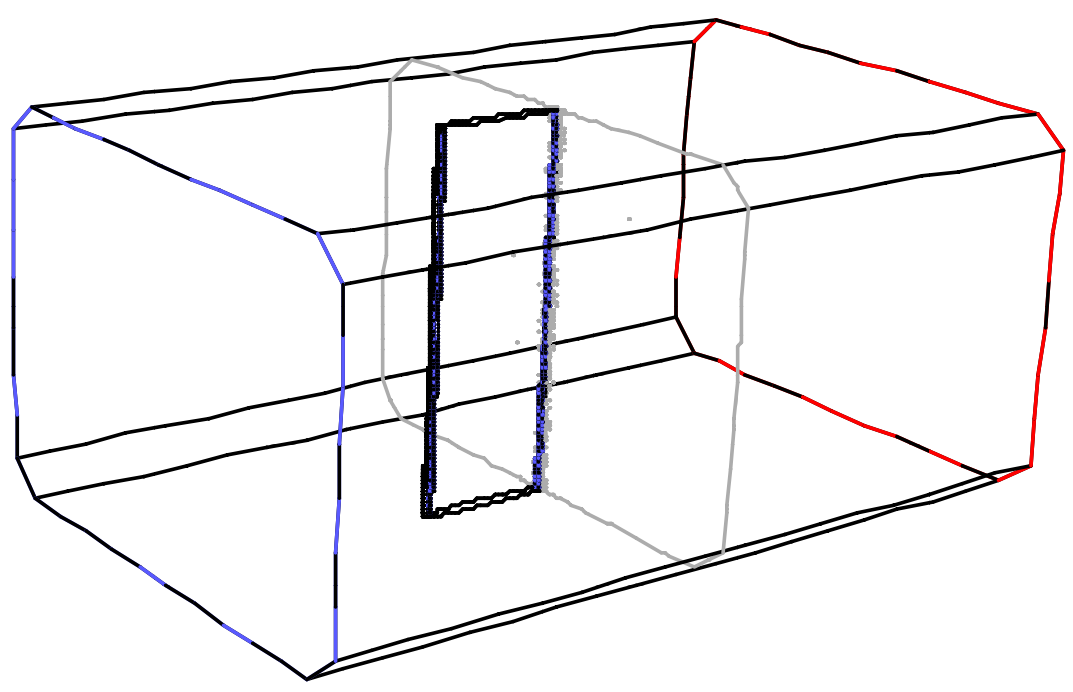

Figure 6.8: Extents of three-dimensional volume for the 0 degree sweep CCHB analysis 
A 45 degree swept wing case was developed by rotating and centering the model along the length of the computational test section as shown in Figure 6.9. The length of the wing section was extended to $451 / 4$ inches, so that the wing spanned the 32 inch height of the computational space. Both the normal and 45 degree swept computational models utilized the same boundary conditions, a velocity inlet to the left, a pressure outlet to the right, and the remaining exterior surfaces were set as walls. The blowing slots on the wing were set as velocity inlets and the remaining airfoil surfaces were set as walls.

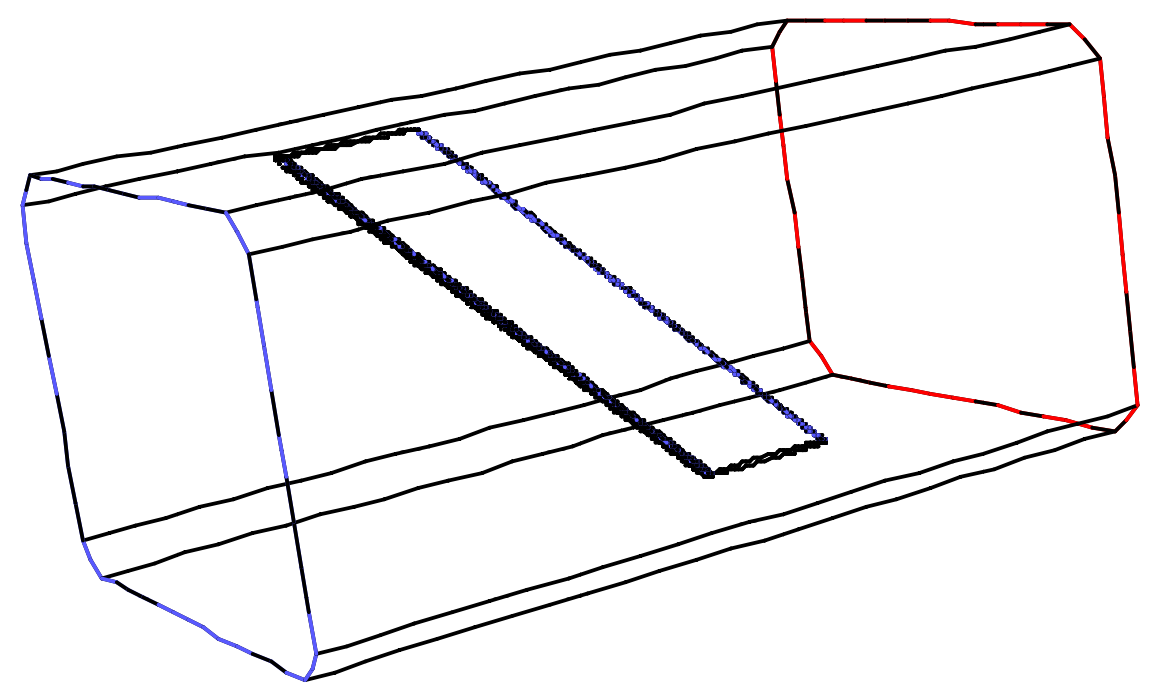

Figure 6.9: Extents of three-dimensional volume for the 45 degree swept CCHB analysis

The computational grids for airfoil surface for both of the three-dimensional cases ( 0 and 45 degrees) were based on the grid from the two-dimensional geometries for the spacing along the chord length of the wing. The vertical spacing of the grid points was set such that a triangular mesh on the surface of the model would have equal sizes. Thus, since the spacing along the chordline was skewed toward the blowing slots, the span-wise distribution was closer near the slot than near the mid-chord of the airfoil. A growth rate of 1.5 was used to expand the grid away from surface of the wing. 


\subsection{Computational Solver Specifics}

As with any computational investigation there are a multitude of options that can be used in the settings for the solver. With the different computational models used here, slight differences in the settings were used.

\subsubsection{Two-Dimensional Solver Settings}

The two-dimensional computational cases were run in three distinct steps. The first of these steps was to solve the case using the steady state, laminar turbulence model with no circulation control blowing in Fluent for the $82 \mathrm{ft} / \mathrm{s}$ freestream velocity. The incompressible flow solver was used for this preliminary study with the convergence criteria for the laminar cases set to:

- $1 \times 10^{-3}$ for continuity

- $1 \times 10^{-3}$ for $\mathrm{x}$-velocity

- $1 \times 10^{-3}$ for $\mathrm{y}$-velocity

- $1 \times 10^{-6}$ for energy

The accuracy settings for the solver in the laminar cases were:

- $2^{\text {nd }}$ Order for Pressure

- $2^{\text {nd }}$ Order Upwind for Momentum

- $2^{\text {nd }}$ Order Upwind for Energy

After the laminar case converged, the turbulence model was changed to the two equation k- $\omega$ model. From previous computational experience, see Angle et. al. [2005], the continuity is typically the last parameter to converge. Thus, to obtain a more converged value, the continuity convergence criteria was changed to $1 \times 10^{-4}$. The $\mathrm{k}-\omega$ turbulence model was run for the steady state, non-blowing case with a freestream velocity of $82 \mathrm{ft} / \mathrm{s}$ with $1^{\text {st }}$ order upwind settings for the turbulent kinetic energy and the specific dissipation rate. 
The final step in the solution was to use the non-blowing case as the initial flowfield for the unsteady solution with the various combinations of the blowing conditions as listed in

Table 6.1. The freestream velocity of $82 \mathrm{ft} / \mathrm{s}$ was used for the majority of the cases, for similarity to the experimental test conditions.

Table 6.1: Major parameters used for computational analysis

\begin{tabular}{|c|c|c|c|c|}
\hline Parameter & Units & $\begin{array}{c}\text { Minimum } \\
\text { Value }\end{array}$ & Increment & $\begin{array}{c}\text { Maximum } \\
\text { Value }\end{array}$ \\
\hline Leading Edge Blowing Velocity & $\mathrm{ft} / \mathrm{s}$ & 200 & 200 & 1000 \\
\hline Trailing Edge Blowing Velocity & $\mathrm{ft} / \mathrm{s}$ & 400 & 200 & 1600 \\
\hline Freestream Velocity & $\mathrm{ft} / \mathrm{s}$ & 450 & 150 & 1500 \\
\hline
\end{tabular}

Each of the two-dimensional computational cases for the circulation control blowing with the unsteady solver were run to allow for a prediction of the force establishment time requirement variation with blowing conditions. Time steps of one millisecond were used in this analysis to adequately capture the time needed to change the force created by the airfoil.

\subsubsection{Three-Dimensional Solver Settings}

For this preliminary investigation into the influence of sweep on the effectiveness of circulation control the same computational settings from the two-dimensional settings were utilized. Only one blowing condition, trailing edge upper surface blowing coefficient of 0.52 , was investigated in this analysis.

\subsection{Computational Grid Independence}

As in any computational analysis, the refinement of the computation grid can alter the results. To minimize the mathematical errors from the grid size, a grid independence study was performed. For this analysis the wind tunnel model was computationally modeled with varying interval size on the airfoil surface and varying growth rates of the 
mesh from the airfoil surface. The various combinations of interval size and growth rates resulted in meshes of various sizes, ranging from 500,000 nodes to 1.8 million nodes in the three-dimensional flow field.

The lift and drag coefficients for the solved cases are shown in Table 6.2, Figure 6.10, and Figure 6.11, respectively. The growth rates were defined for the inner and outer regions of the computational space. For the data cases, the two regions of the flow field were given the same growth rate. The data from the laminar cases showed that the model interval size, on surfaces outside the blowing slot, of 0.1 was inadequate for this analysis, due to the considerably lower lift coefficient and thus the turbulent models were not run with this spacing. Similarly, the 0.05 spacing modeled with the k- $\omega$ turbulence model only changed the lift coefficient by $3 \%$ from the 0.075 spacing.

From this grid independence study, it was determined that a spacing of 0.075 (nominally $1 \times 10^{6}$ node points) would yield quantitative results with minimal computational time requirements. A growth rate of 1.5 was used to expand the mesh on the surface to fill the flow field, as described in Section 6.1. Figure 6.12 defines the location of the boundary between the inner and outer regions for expanding the growth rate away from the airfoil.

Table 6.2: Summary of the data collected for the grid independence study

\begin{tabular}{|c|c|c|c|c|c|c|c|c|c|}
\hline \multirow{2}{*}{$\begin{array}{l}\text { Interval } \\
\text { Size on } \\
\text { Model } \\
\text { Surface }\end{array}$} & \multirow[t]{2}{*}{$\begin{array}{l}\text { Inner } \\
\text { Growth } \\
\text { Rate }\end{array}$} & \multirow[t]{2}{*}{$\begin{array}{c}\text { Outer } \\
\text { Growth } \\
\text { Rate }\end{array}$} & \multirow{2}{*}{$\begin{array}{c}\text { Three- } \\
\text { Dimensional } \\
\text { Computational } \\
\text { Node Points }\end{array}$} & \multicolumn{2}{|c|}{$10^{\wedge}-3 r d$ Laminar } & \multicolumn{2}{|c|}{$\begin{array}{c}10^{\wedge}-3 \mathrm{rd} \mathrm{K}- \\
\text { Omega }\end{array}$} & \multicolumn{2}{|c|}{$\begin{array}{l}10^{\wedge}-4 \text { th K- } \\
\text { Omega }\end{array}$} \\
\hline & & & & $\mathrm{C}_{\mathrm{I}}$ & $\mathrm{C}_{\mathrm{d}}$ & $\mathrm{C}_{\mathrm{l}}$ & $\mathrm{C}_{\mathrm{d}}$ & $\mathrm{C}_{\mathrm{I}}$ & $\mathrm{C}_{\mathrm{d}}$ \\
\hline 0.05 & 1.3 & 1.5 & $1,810,393$ & & & 2.057 & 0.298 & & \\
\hline 0.05 & 1.5 & 1.5 & $1,486,826$ & 2.36 & 0.187 & & & & \\
\hline 0.05 & 1.5 & 1.7 & $1,479,149$ & 2.357 & 0.188 & & & & \\
\hline 0.075 & 1.3 & 1.3 & $1,507,007$ & 2.509 & 0.178 & 1.919 & 0.279 & 1.819 & 0.281 \\
\hline 0.075 & 1.5 & 1.7 & $1,184,015$ & 2.779 & 0.209 & 2.032 & 0.314 & 1.967 & 0.317 \\
\hline 0.075 & 1.7 & 1.9 & $1,063,082$ & 2.736 & 0.216 & 2.035 & 0.322 & 1.982 & 0.324 \\
\hline 0.1 & 1.3 & 1.3 & 508,907 & 1.898 & 0.134 & & & & \\
\hline 0.1 & 1.3 & 1.5 & 506,570 & 1.897 & 0.134 & & & & \\
\hline 0.1 & 1.3 & 1.7 & 505,692 & 1.897 & 0.134 & & & & \\
\hline
\end{tabular}




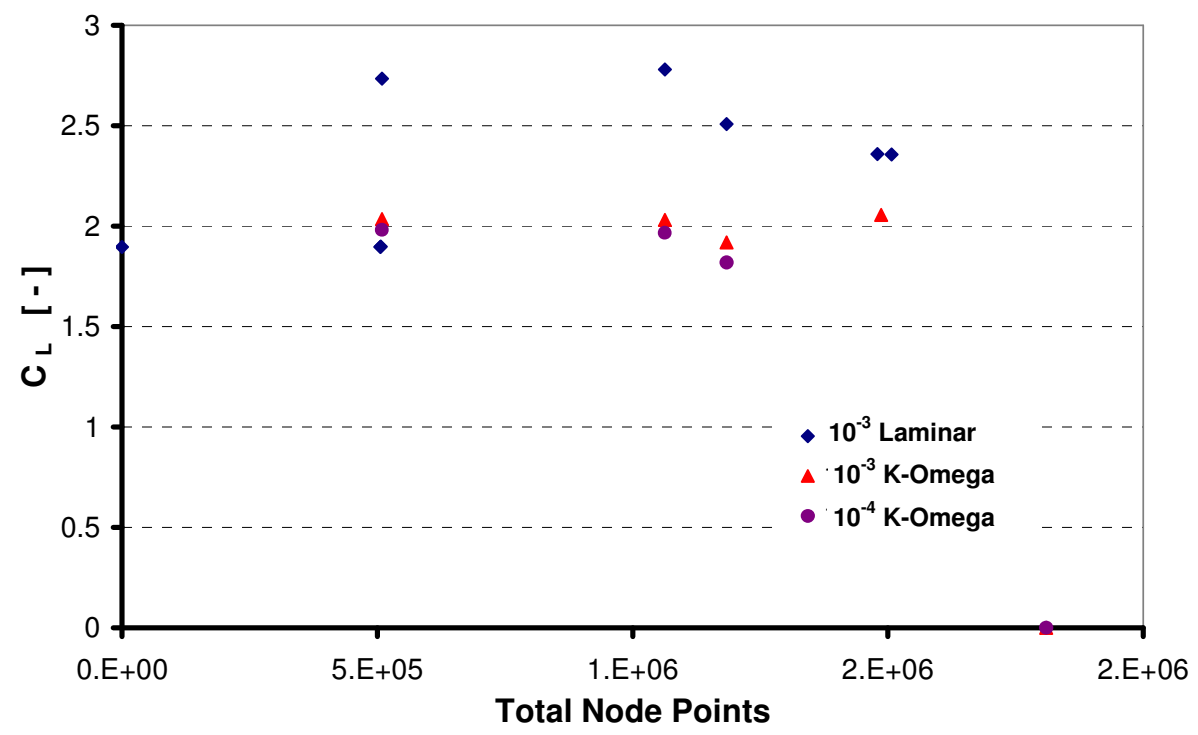

Figure 6.10: Variation in lift coefficient with respect to the number of computational node points

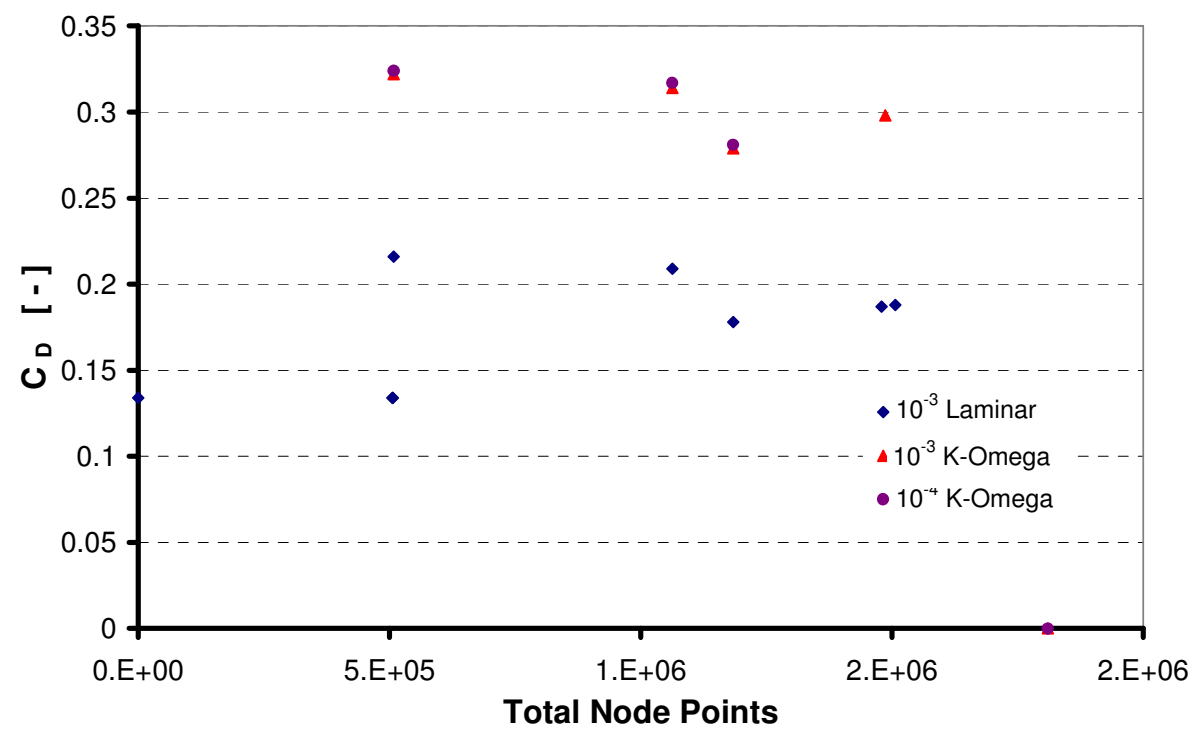

Figure 6.11: Variation in drag coefficient with respect to the number of computational node points 


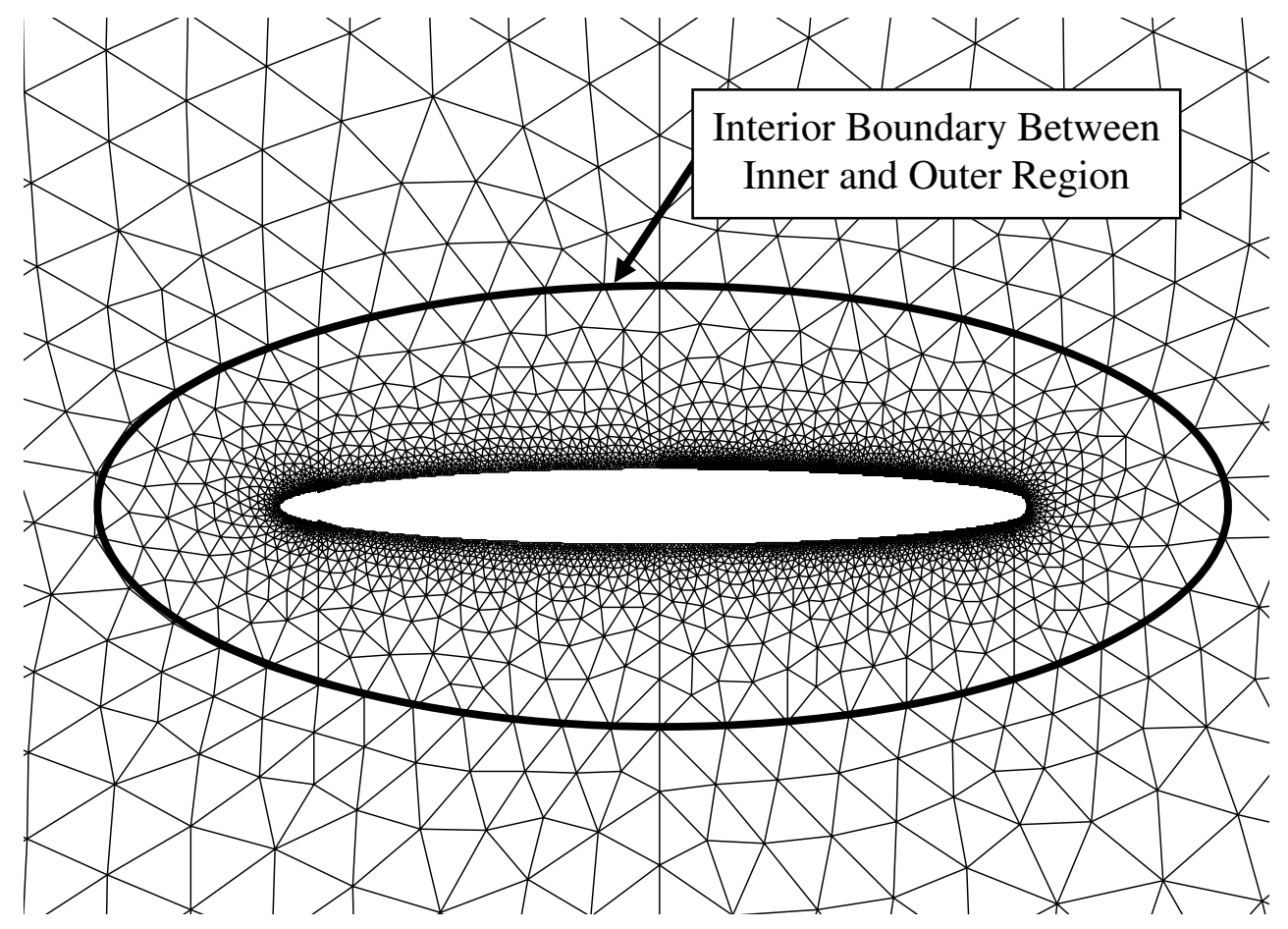

Figure 6.12: View of computational grid near the airfoil

\subsection{Computational Results}

The computational analysis was used to expand the conditions investigated experimentally. An expanded jet velocity range allowed for investigation into higher Reynolds numbers, and blowing coefficients. The lift and drag coefficient were determined based on the force value and inlet velocity and the computational results were found to over predict the lift augmentation at low blowing coefficients, compared to the experimental data. Applying a linear fit to the CFD data revealed an $8 \%$ lower slope for the computational fluid dynamics results than the experimental data

\subsubsection{Two-Dimensional Results}

A total of 99 different combinations of blowing conditions, up to a total blowing coefficient (sum of leading and trailing edge) of 0.8 , were computationally solved in this investigation, a summary of the results are shown in this section. Figure 6.13 shows the streamlines determined for the trailing edge upper blowing coefficient of 0.36 , and 0 degrees angle of attack. The circulation control jet has moved the separation point to the 
lower surface of the airfoil, which resulted in a lift coefficient 3.80 and a drag coefficient of 1.54. The lift and drag coefficients for the various trailing edge blowing coefficients are listed in Table 6.3. The computational trailing edge blowing results when compared to the Kind and Maull [1968] data, as in Figure 6.14, reveal a slight over-prediction $\left(C_{1}\right.$ of 0.3) of the of the lift augmentation for the 0.49 blowing coefficient case. The blowing coefficient was changed by increasing the blowing velocity with an $80 \mathrm{ft} / \mathrm{s}$ freestream velocity. For this initial study, the blowing coefficient has been emphasized thus compressibility effects have been neglected; high blowing coefficients can also be achieved by reducing the freestream velocity.

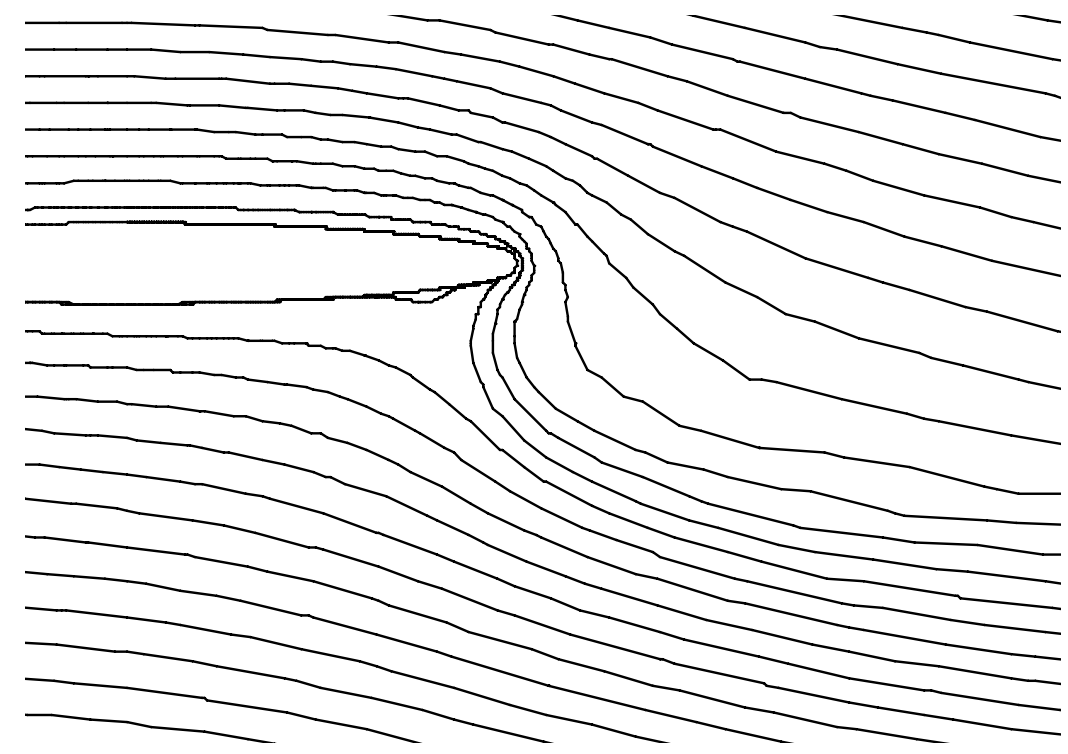

Figure 6.13: Trailing edge streamlines for trailing edge upper jet $C_{\mu}=0.36$ and freestream velocity of $82 \mathrm{ft} / \mathrm{s}$

Table 6.3: Summary of the trailing edge, upper surface blowing computational results as found for a freestream velocity of $80 \mathrm{ft} / \mathrm{s}$

\begin{tabular}{|c|c|c|}
\hline $\begin{array}{c}\text { Blowing } \\
\text { Coefficient }\end{array}$ & $\begin{array}{c}\text { Lift } \\
\text { Coefficient }\end{array}$ & $\begin{array}{c}\text { Drag } \\
\text { Coefficient }\end{array}$ \\
\hline- & - & - \\
\hline 0 & 0.089 & 0.022 \\
\hline 0.0403 & 1.622 & 0.339 \\
\hline 0.0907 & 2.242 & 0.56 \\
\hline 0.1612 & 2.783 & 0.831 \\
\hline 0.2519 & 3.296 & 1.158 \\
\hline 0.3628 & 3.802 & 1.539 \\
\hline 0.4938 & 4.316 & 1.975 \\
\hline 0.645 & 4.848 & 2.472 \\
\hline
\end{tabular}


To investigate the symmetric nature of the blowing, identical conditions were solved for the lower surface trailing edge blowing conditions, summarized in Table 6.4. The lift coefficients for the lower surface blowing cases were found to be within $4 \%$ of the upper surface blowing cases, the drag coefficients were found to be within 5\% difference. The primary difference between the upper and lower surface blowing is that the gravitational acceleration was defined to be in the negative y direction. Thus, the small difference between the upper and lower surface blowing were due to the flow interaction with the leading and trailing edge jets. Figure 6.14 compares computational data to the historical data from Kind and Maull [1968] and the experimental data collected during this investigation. The incompressible model resulted in a transition point at a blowing coefficient of 0.012 . Prior to this transition point the lift augmentation ratio was found to be 85 , which changed to 12 after the transition point. The computational model resulted in an overall lift augmentation ratio of 8.3 over the entire range of blowing coefficients investigated.

Table 6.4: Summary of the trailing edge, lower surface blowing computational results as found for a freestream velocity of $80 \mathrm{ft} / \mathrm{s}$

\begin{tabular}{|c|c|c|}
\hline $\begin{array}{c}\text { Blowing } \\
\text { Coefficient }\end{array}$ & $\begin{array}{c}\text { Lift } \\
\text { Coefficient }\end{array}$ & $\begin{array}{c}\text { Drag } \\
\text { Coefficient }\end{array}$ \\
\hline- & - & - \\
\hline 0 & 0.089 & 0.022 \\
\hline 0.0403 & -1.689 & 0.355 \\
\hline 0.0907 & -2.286 & 0.578 \\
\hline 0.1612 & -2.789 & 0.848 \\
\hline 0.2519 & -3.284 & 1.174 \\
\hline 0.3628 & -3.76 & 1.554 \\
\hline 0.4938 & -4.229 & 1.995 \\
\hline 0.645 & -4.706 & 2.488 \\
\hline
\end{tabular}




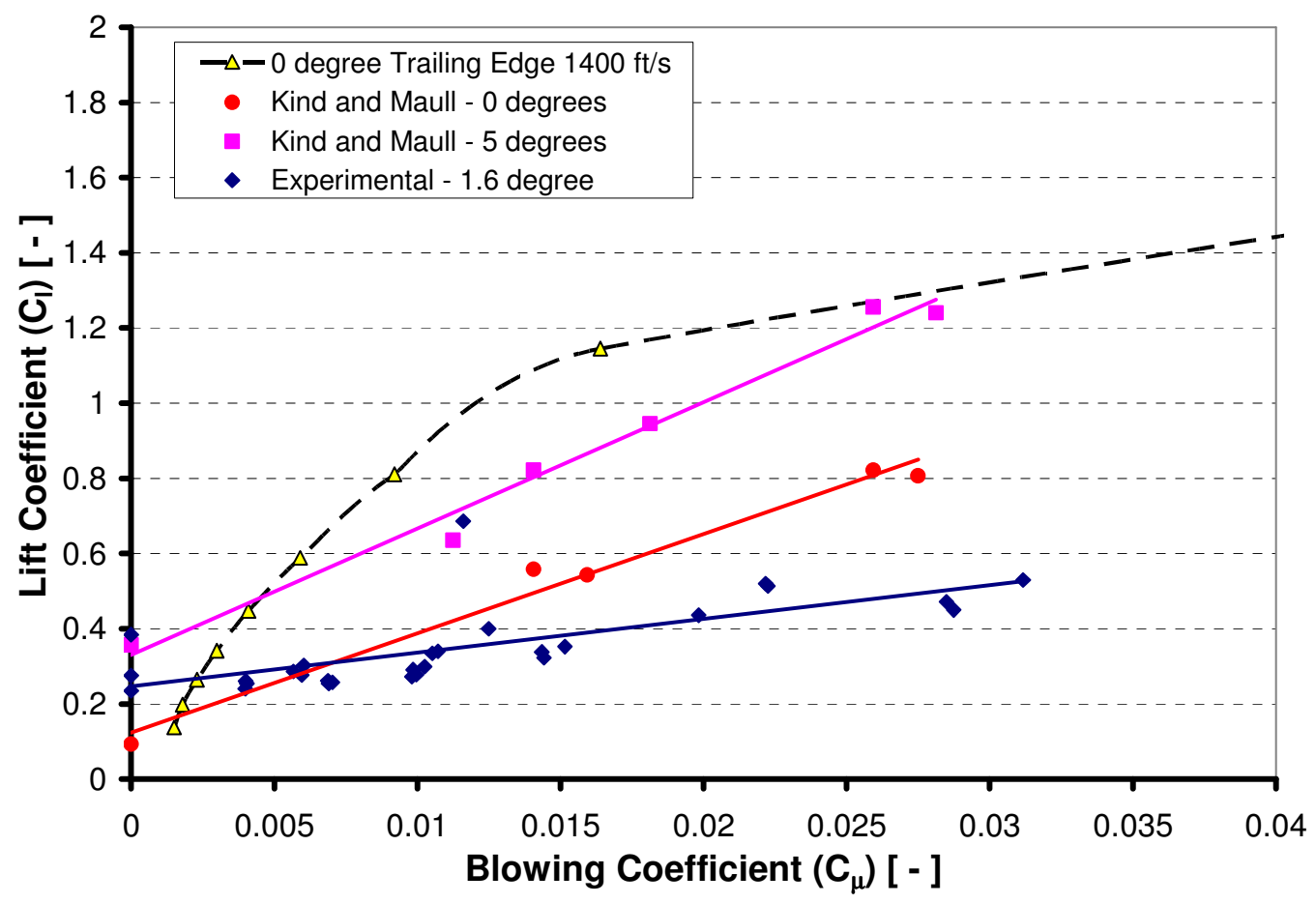

Figure 6.14: Comparison of computational lift coefficient for the trailing edge blowing to the experimental and Kind and Maull [1968] data

The leading edge blowing slot was found to have a smaller influence on the streamlines around the airfoil. Figure 6.15 illustrates the streamlines near the leading edge of the airfoil for the leading edge, upper surface blowing jet velocity of $800 \mathrm{ft} / \mathrm{s}$ with a free stream velocity of $82 \mathrm{ft} / \mathrm{s}$. A better visualization of the flow near the upper surface blowing slot is shown in Figure 6.16, the velocity contour plot. From this figure it is seen that the jet stays within close proximity of the surface and re-energizes the boundary layer, thus acting as a boundary layer control. 


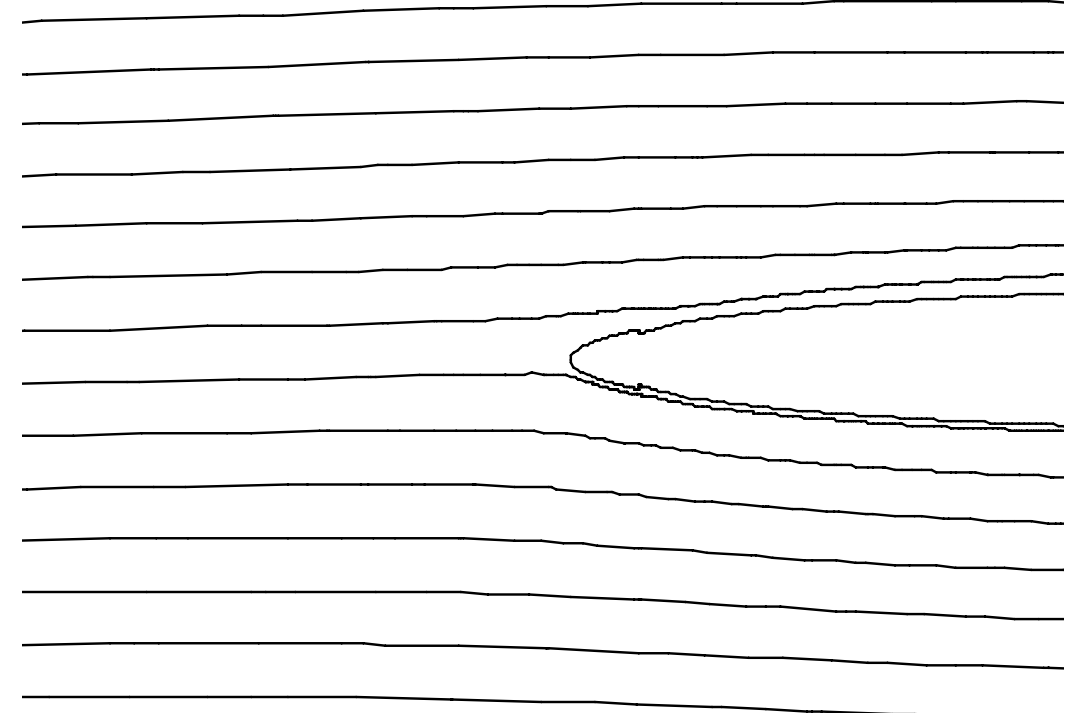

Figure 6.15: Leading edge streamlines for leading edge upper jet $C_{\mu}$ of 0.16 and freestream velocity of $82 \mathrm{ft} / \mathrm{s}$

Looking at the streamline plots of cases combining the leading edge and trailing edge blowing revealed that the trailing edge blowing is a more dominant flow structure. When the leading edge blowing is on the same side, upper surface, of the airfoil the streamlines were found to be more like the trailing edge, Figure 6.17 shows the streamlines for the case with upper surface leading edge blowing of $600 \mathrm{ft} / \mathrm{s}$ $\left(\mathrm{C}_{\mu \_L E}=0.027\right)$ and trailing edge blowing at $1400 \mathrm{ft} / \mathrm{s}\left(\mathrm{C}_{\mu \_\mathrm{TE}}=0.148\right)$ and a freesteam velocity of $150 \mathrm{ft} / \mathrm{s}$. Increasing the leading edge blowing coefficient was found to increase the lift coefficient, by creating a lower upper surface pressure over a majority of the surface. Figure 6.18 and Table 6.5 show that a lift coefficient 4.70 was found for the $600 \mathrm{ft} / \mathrm{s}$ leading edge and $1400 \mathrm{ft} / \mathrm{s}$ trailing edge blowing, a $9 \%$ increase over the lift coefficient of 4.32 found for the trailing edge only blowing condition. 


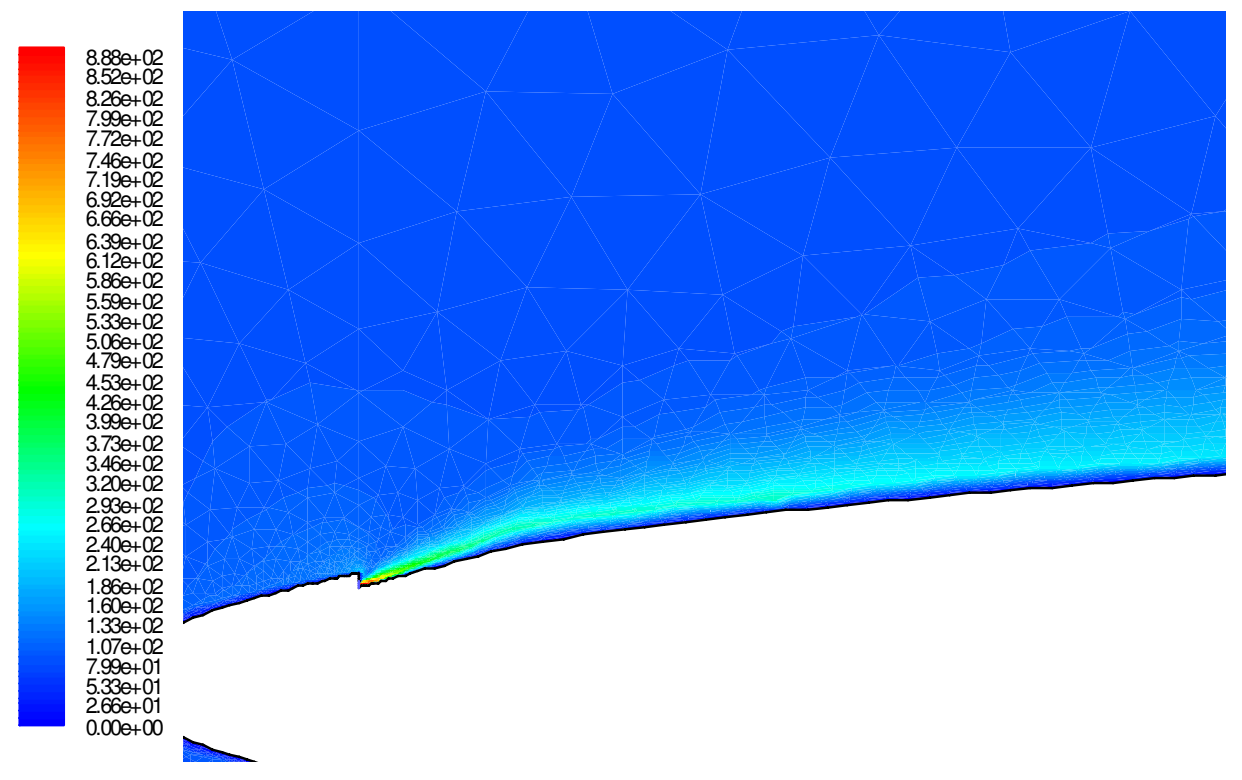

Figure 6.16: Velocity contours near the leading edge of the CCHB airfoil with leading edge upper surface blowing coefficient of 0.16

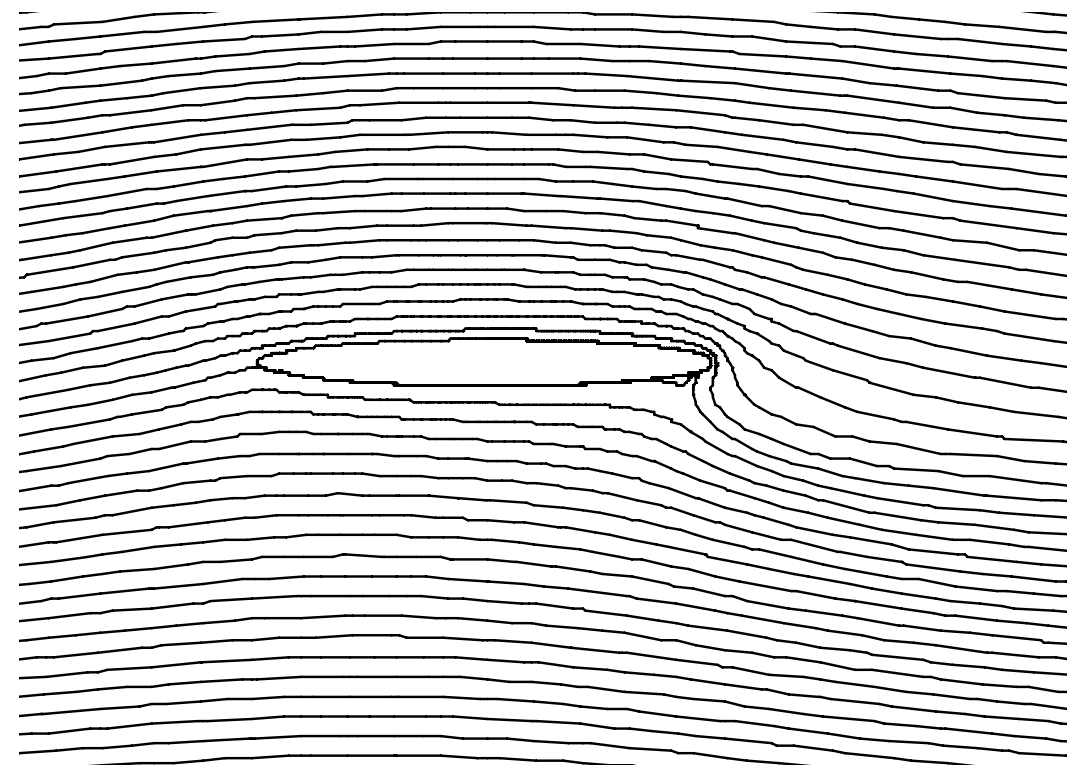

Figure 6.17: Streamlines for the leading edge blowing at $C_{\mu \_L E}=0.027$ and trailing edge blowing at $\mathrm{C}_{\mu \_\mathrm{TE}}=0.148$ 


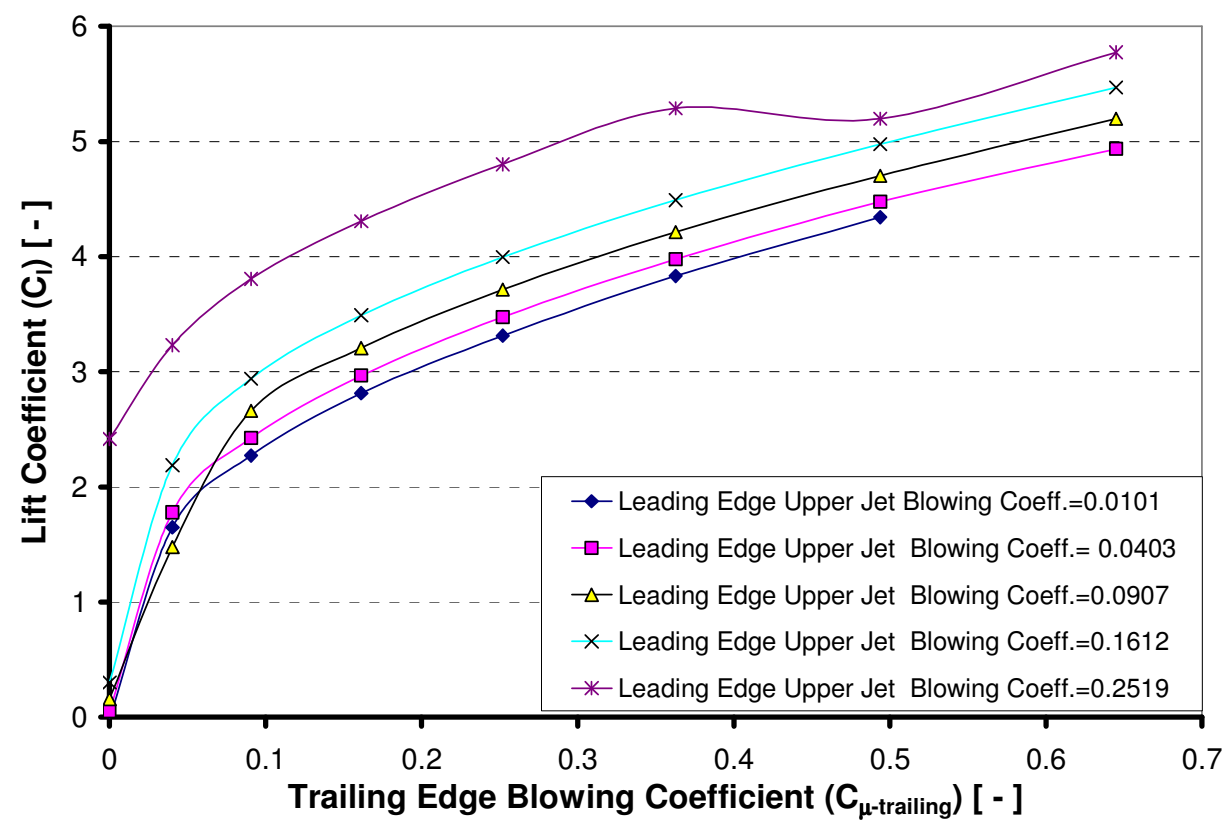

Figure 6.18: Computational lift coefficient for various leading edge, upper surface blowing coefficient as dependent upon the trailing edge, upper surface blowing coefficient

Table 6.5: Summary of leading edge upper surface blowing at $C_{\mu \_L E}$ of 0.091 with a freestream velocity of $80 \mathrm{ft} / \mathrm{s}$ cases

\begin{tabular}{|c|c|c|c|}
\hline $\begin{array}{c}\text { Trailing Edge } \\
\text { Jet Velocity }\end{array}$ & $\begin{array}{c}\text { Blowing } \\
\text { Coefficient }\end{array}$ & $\begin{array}{c}\text { Lift } \\
\text { Coefficient }\end{array}$ & $\begin{array}{c}\text { Drag } \\
\text { Coefficient }\end{array}$ \\
\hline $\mathrm{ft} / \mathrm{s}$ & - & - & - \\
\hline 0 & 0 & 0.158 & 0.186 \\
\hline 400 & 0.0403 & 1.478 & 0.352 \\
\hline 600 & 0.0907 & 2.661 & 0.725 \\
\hline 800 & 0.1612 & 3.206 & 1.011 \\
\hline 1000 & 0.2519 & 3.712 & 1.347 \\
\hline 1200 & 0.3628 & 4.212 & 1.735 \\
\hline 1400 & 0.4938 & 4.702 & 2.179 \\
\hline 1600 & 0.645 & 5.198 & 2.681 \\
\hline
\end{tabular}

Further investigation of the leading edge upper surface blowing coefficient of 0.25 curve from Figure 6.18 shows the compressibility effects for the higher trailing edge blowing coefficients. The value of the lift coefficient is already above four and thus above the lift coefficient of the current swash-plate system. Future investigations should 
incorporate compressibility effects in the prediction of lift and drag performance of the circulation control system.

When the leading edge blowing slot is opposite of the trailing edge blowing, the increase in lift is less dependent upon the leading edge blowing coefficient. As shown in Figure 6.19, when the trailing edge blowing coefficient is less than 0.15 the lift coefficient is reduced as the leading edge blowing velocity is increased. At trailing edge blowing coefficients above 0.15 , increasing the lower surface, leading edge blowing increases the lift coefficient to 4.40 for the leading edge lower slot velocity of 600 $\mathrm{ft} / \mathrm{s}\left(\mathrm{C}_{\mu_{-} \mathrm{LE}}=0.0907\right)$ and trailing edge upper slot velocity of $1400 \mathrm{ft} / \mathrm{s}\left(\mathrm{C}_{\mu_{-} \mathrm{TE}}=0.4938\right)$, a $2 \%$ increase over the trailing edge only blowing case.

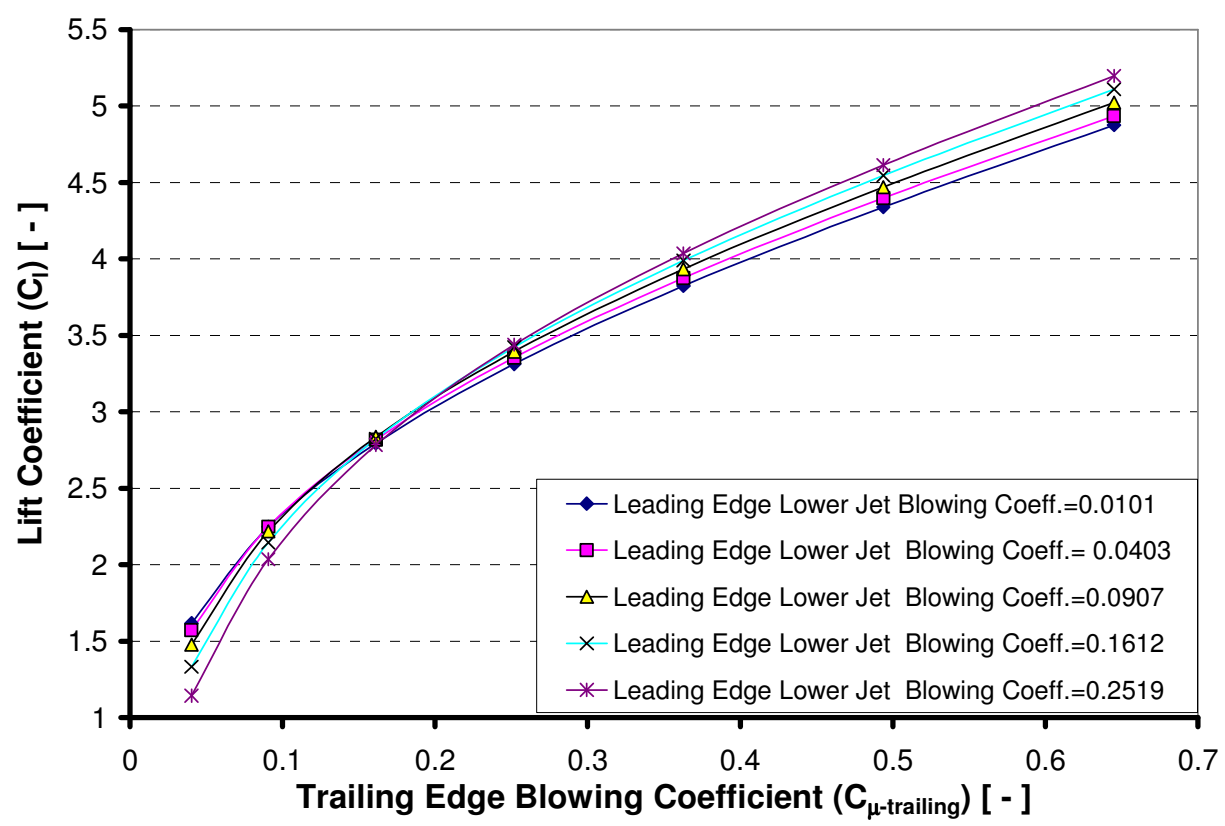

Figure 6.19: Computational lift coefficient for various leading edge, lower surface blowing coefficient as dependent upon the trailing edge, upper surface blowing coefficient

Combining the momentum added through both blowing slots into a total blowing coefficient shows a limit to the combined effects for the leading and trailing edge, upper surface blowing slots, as shown in Figure 6.20. Increasing the leading edge blowing coefficient has less influence on the lift coefficient than the trailing edge blowing coefficient. Equation 6.2 shows the predicted limit to the lift coefficient determined from 
fitting a curve to the overlapping curves in Figure 6.20 for total blowing coefficients less than 0.80 .

$$
C_{\ell}=-2.3497 C_{\mu}^{2}+6.3952 C_{\mu}+1.8108
$$

Equation 6.2

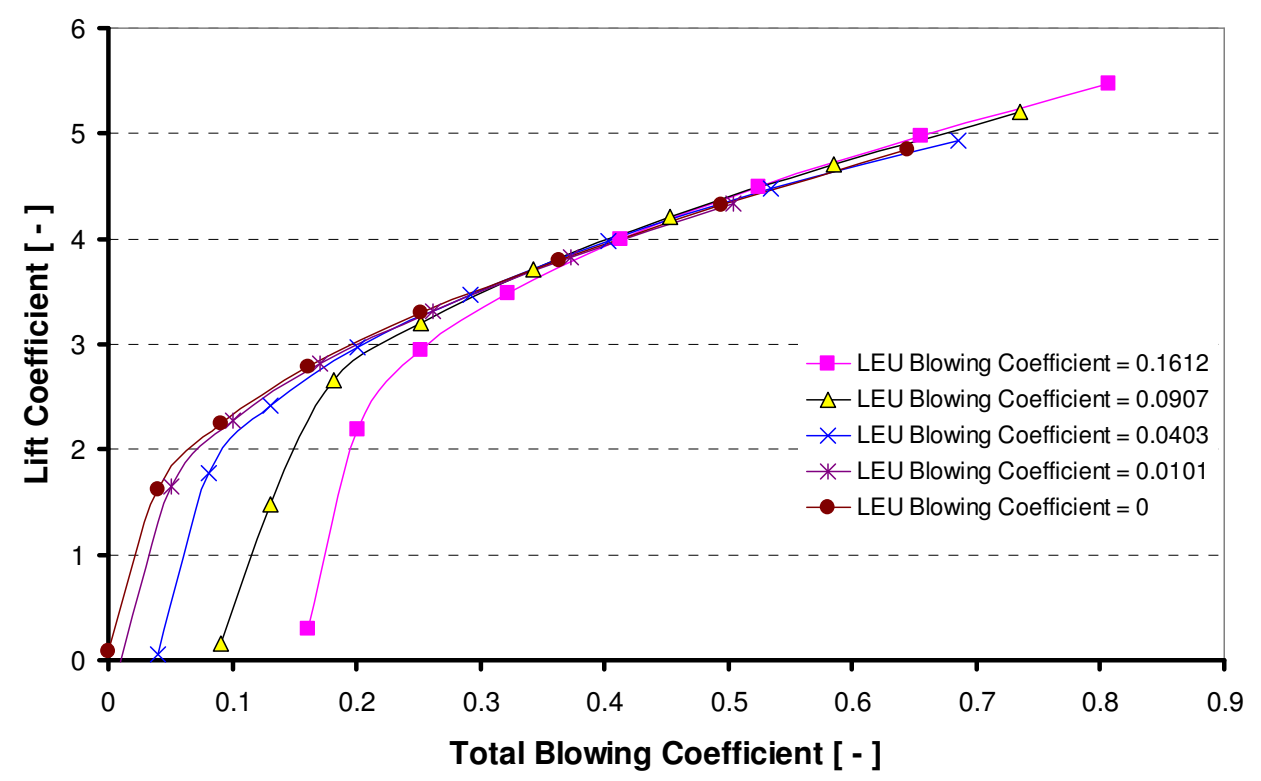

Figure 6.20: Computational lift coefficient for total blowing coefficient with leading and trailing edge, upper surface blowing

Changes in the freestream velocity were found to impact the lift coefficient for constant blowing jet velocities, due to the resulting changes in the blowing coefficients. As the momentum of the freestream is increased, with higher velocities, the blowing coefficient in decreased; thus a proportional increase in jet velocity is needed to maintain circulation control performance. Figure 6.21 shows that with jet velocities equal or lower than the freestream velocity has little effect on the streamlines, which resembles the streamlines of an airfoil without circulation control. For a trailing edge jet velocity of $1400 \mathrm{ft} / \mathrm{s}$ and a $600 \mathrm{ft} / \mathrm{s}$ leading edge jet velocity the lift coefficient asymptotically decreases with the freestream velocity, as shown in Figure 6.22. This decrease in lift augmentation is as expected since the ratio of jet momentum to the freestream momentum is decreased, reducing the blowing coefficient. To compensate for the increased velocity as radial station is increased the blowing coefficient may need to be a 
function of the radius. This could be tailored through use of a varying slot width versus radial station.

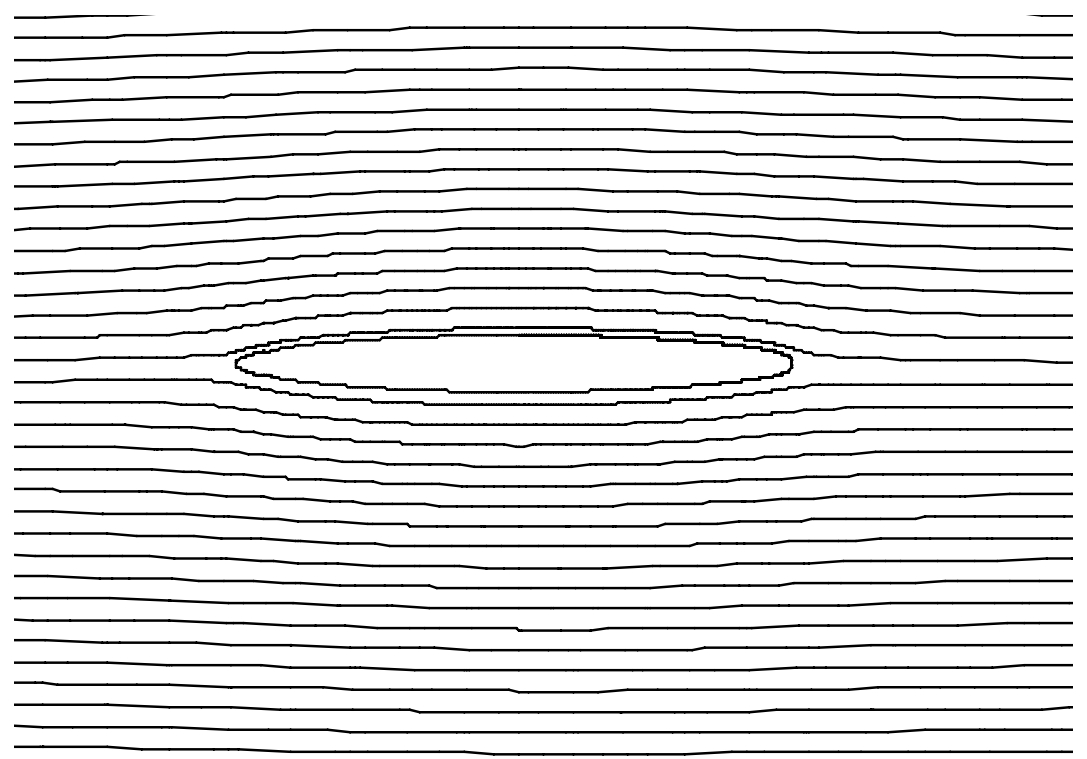

Figure 6.21: Streamlines for freestream velocity of $1500 \mathrm{ft} / \mathrm{s}$, leading edge upper surface blowing at $600 \mathrm{ft} / \mathrm{s}\left(\mathrm{C}_{\mu \_L E}=0.0003\right)$ and trailing edge upper surface blowing at $1400 \mathrm{ft} / \mathrm{s}$ $\left(\mathrm{C}_{\mu \_\mathrm{TE}}=0.001\right)$

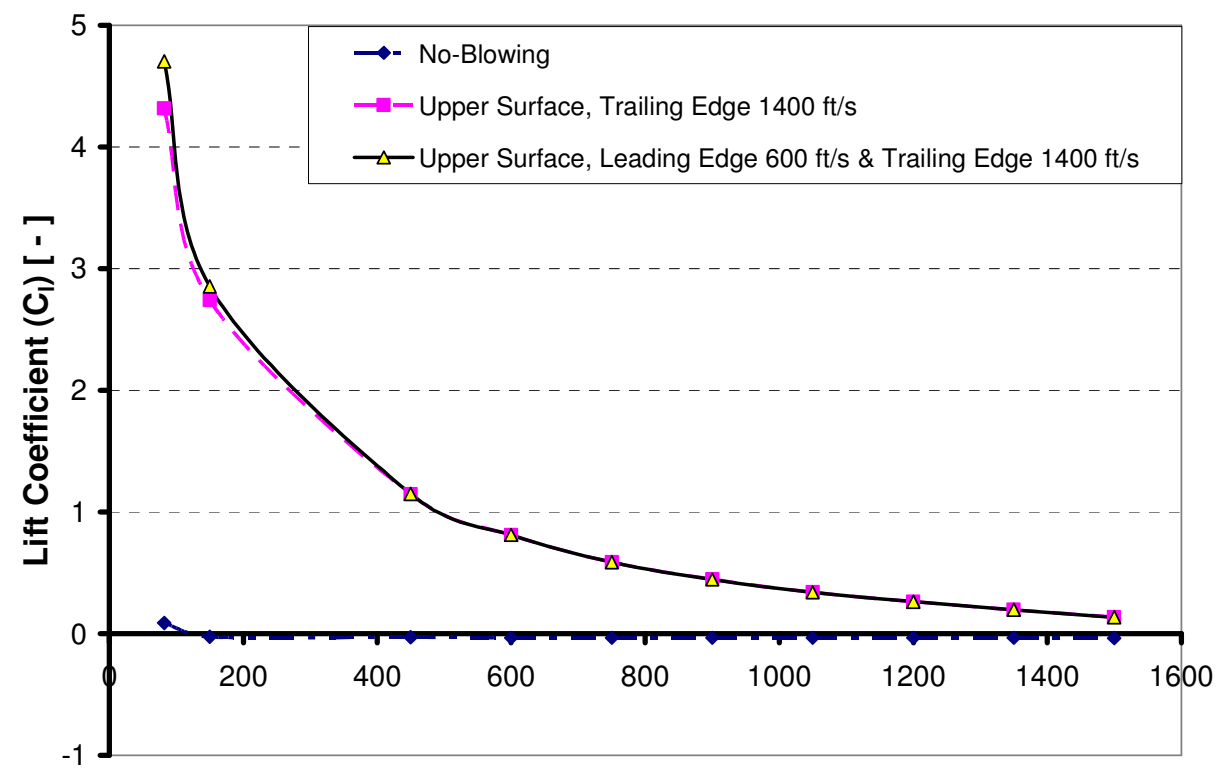

Freestream Velocity [ft/s]

Figure 6.22: Steady state, lift coefficient as dependent upon the freestream velocity for uniform blowing conditions 


\subsubsection{Unsteady Two-Dimensional Time Response Results}

The results from the unsteady computational cases were used to estimate the force augmentation development time. The trailing edge blowing, with a jet velocity of 800 $\mathrm{ft} / \mathrm{s}$, stabilized at the new force values at 0.050 seconds and 0.060 seconds for the lift and drag, respectively as shown in Figure 6.23 and Figure 6.24. It is important to note that the force coefficients listed in these, and subsequent CFD time analysis figures were based on the Fluent default velocity and length scales and not the scales for this particular flow geometry. Since, these figures were used only to determine the rise time of the forces, correcting the magnitude of the lift coefficient was neglected due to the magnitude being independent of time.

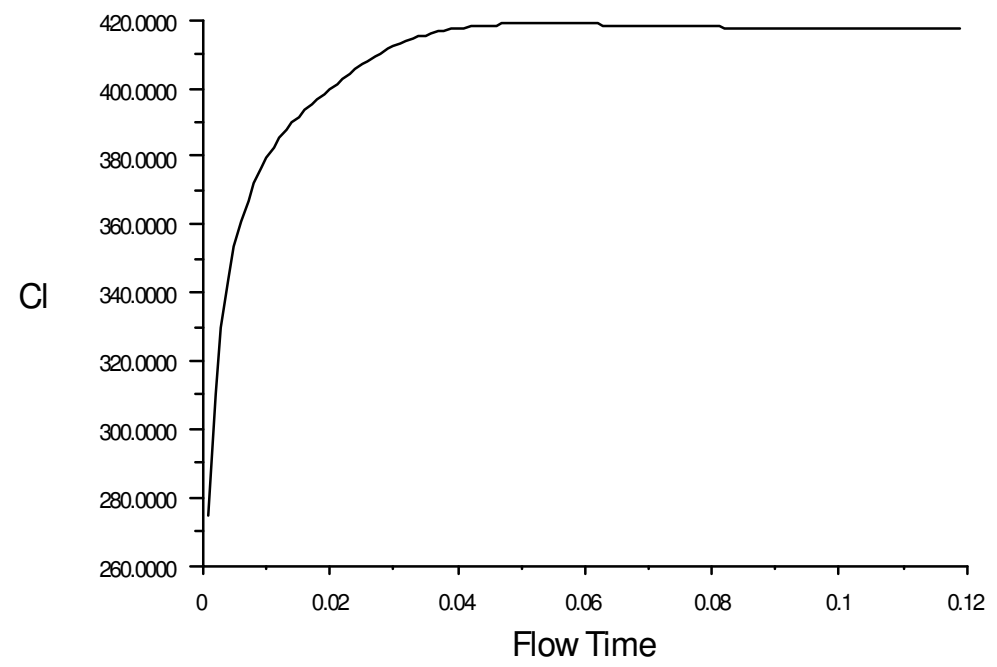

Figure 6.23: Time response for lift with trailing edge upper surface blowing at $C_{\mu}=0.16$ 


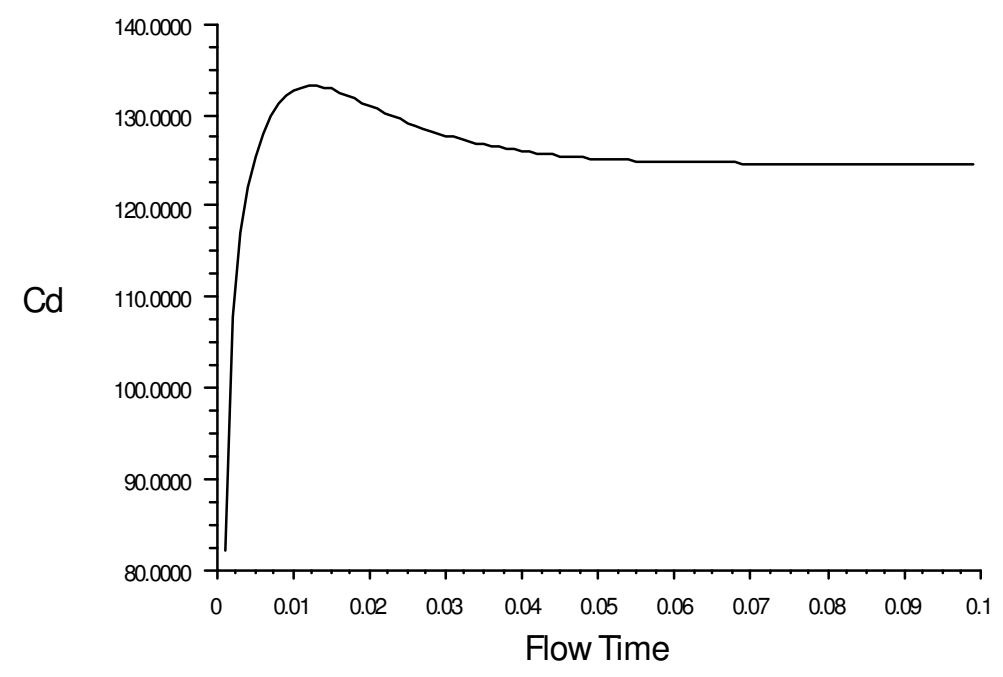

Figure 6.24: Time response for drag with trailing edge upper surface blowing at $C_{\mu}=$ 0.16

The leading edge, upper surface blowing had response time for the lift force of 60 milliseconds, as shown in Figure 6.25, while the drag force, Figure 6.26, stabilized within 25 milliseconds.

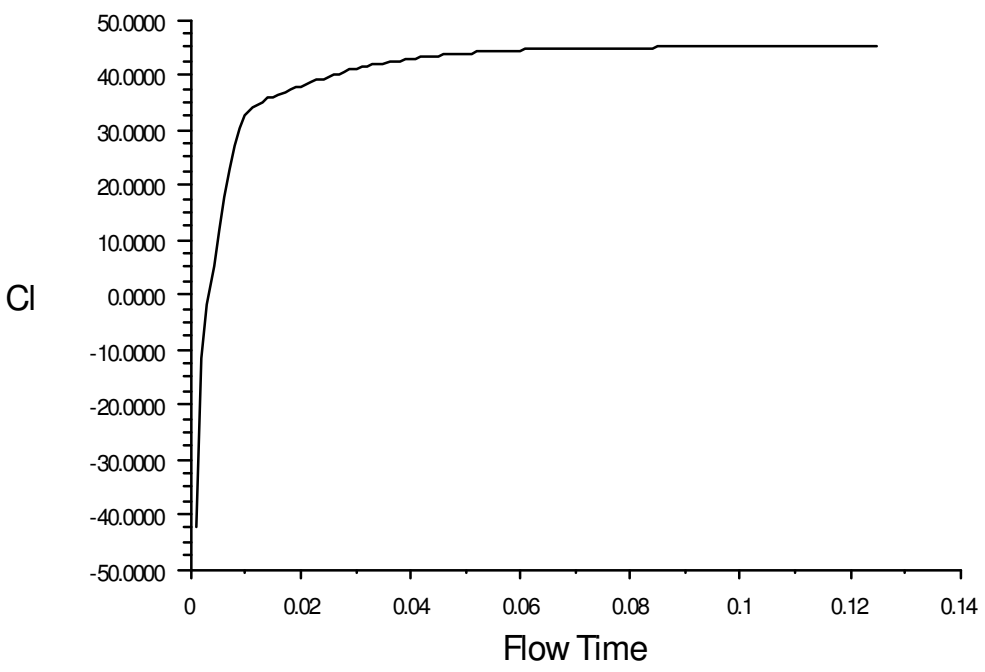

Figure 6.25: Time response for lift with leading edge upper surface blowing at $C_{\mu}=0.16$ 


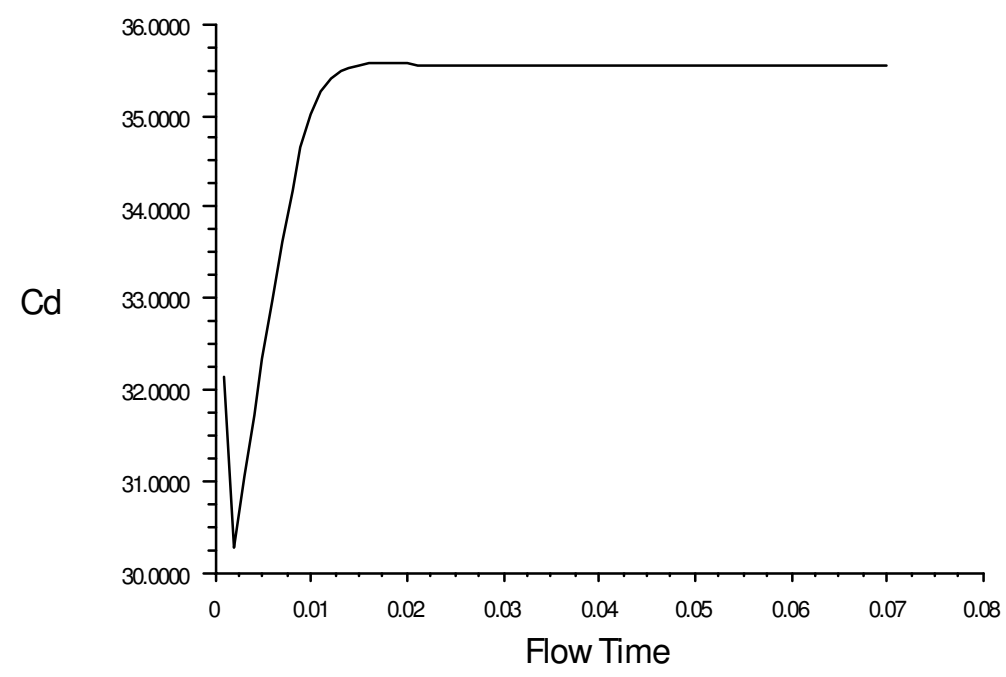

Figure 6.26: Time response for drag with leading edge upper surface blowing at $\mathrm{C}_{\mu}=$ 0.16

Combining the upper surface, leading and trailing edge blowing slots had minimal effect on the time response of establishing the forces. Figure 6.27 shows the response of the lift force to opening the two blowing slots, leading edge at $600 \mathrm{ft} / \mathrm{s}\left(\mathrm{C}_{\mu_{-} L E}=0.09\right)$ and trailing edge at $1400 \mathrm{ft} / \mathrm{s}\left(\mathrm{C}_{\mu_{-} \mathrm{TE}}=0.49\right)$ which responded within 60 milliseconds. The time for the drag to respond was also 60 milliseconds for the dual blowing case, as shown in Figure 6.28. Appendix I shows the curves for the time responses for several other blowing conditions, all of which are predicted to stabilize at the steady state value within 60 milliseconds. However, the lift coefficient reaches $80 \%$ of the steady state value within $15 \mathrm{msec}$ and corresponds to a lift coefficient of over 3.0; still greater than the lift coefficient of the stall angle of the non-CC airfoil. 


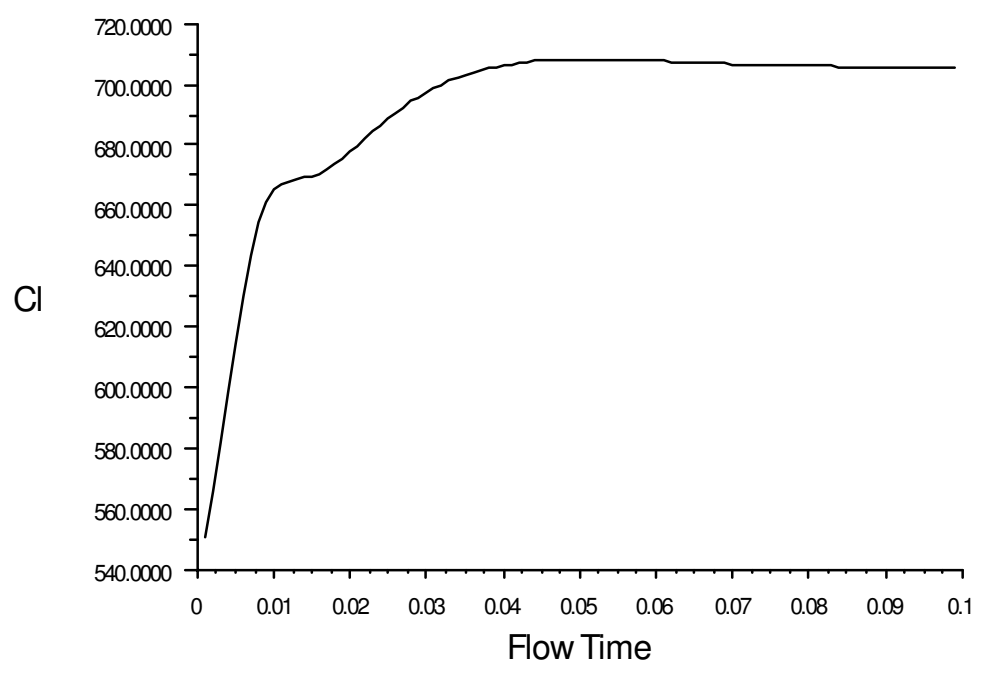

Figure 6.27: Time response for lift with upper surface blowing on both the leading edge at $\mathrm{C}_{\mu \_L E}=0.09$ and the trailing edge at $\mathrm{C}_{\mu_{-} \mathrm{TE}}=0.49$

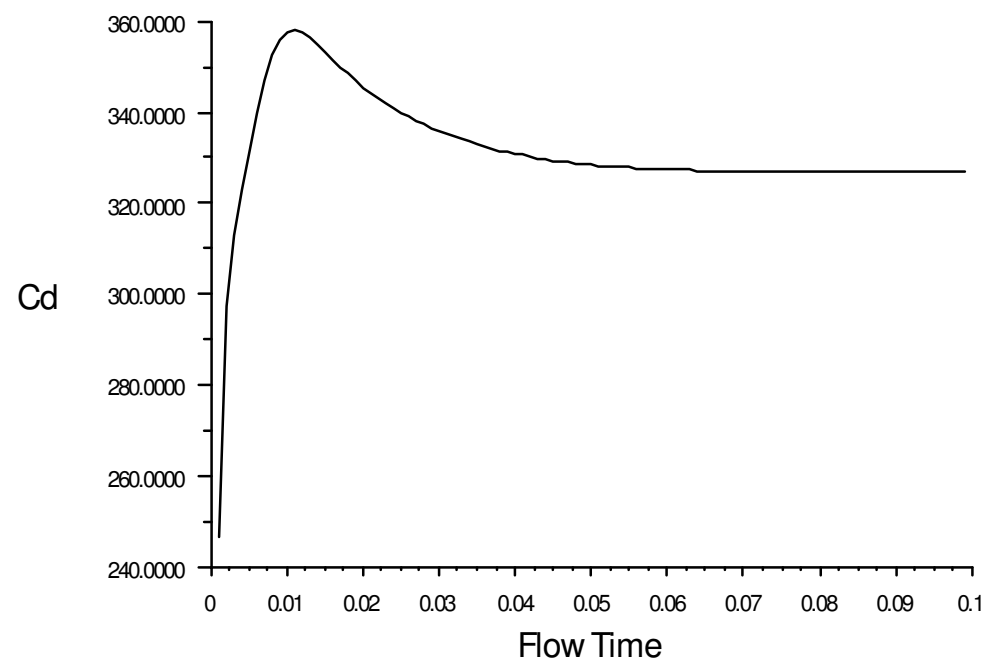

Figure 6.28: Time response for drag with upper surface blowing on both the leading edge at $\mathrm{C}_{\mu_{-} \mathrm{LE}}=0.09$ and the trailing edge at $\mathrm{C}_{\mu_{-} \mathrm{TE}}=0.49$

\subsubsection{Three-Dimensional Results}

The three-dimensional computational analysis of the un-swept and 45 degree sweep cases determined subtle differences in the lift performance of the circulation control blowing. Figure 6.29 shows the velocity contour near the model and along the 
centerline of the model. Comparing this figure to Figure 6.30 for the swept wing, the trailing edge separation of the blown jet occurs earlier for the swept wing. Thus there is a greater circulation around the un-swept case. This is also evident in the lift coefficient results, where a value of 3.08 was found for the un-swept model and 1.41 for the 45 degree swept wing. Applying the sweep theory from Keener, et.al. [1986] to the swept case, a lift coefficient in the normal direction of 2.82 was found; this value is $8 \%$ lower than the un-swept case.

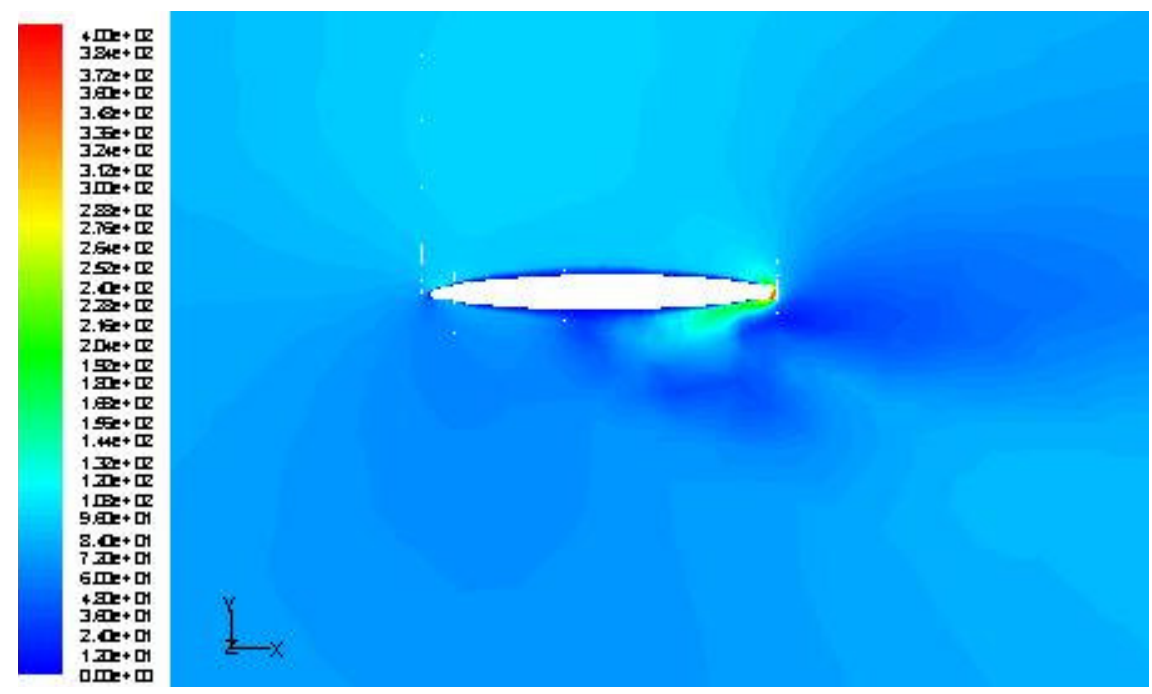

Figure 6.29: Velocity contour along centerline of un-swept geometry with trailing edge blowing coefficient of 0.52 .

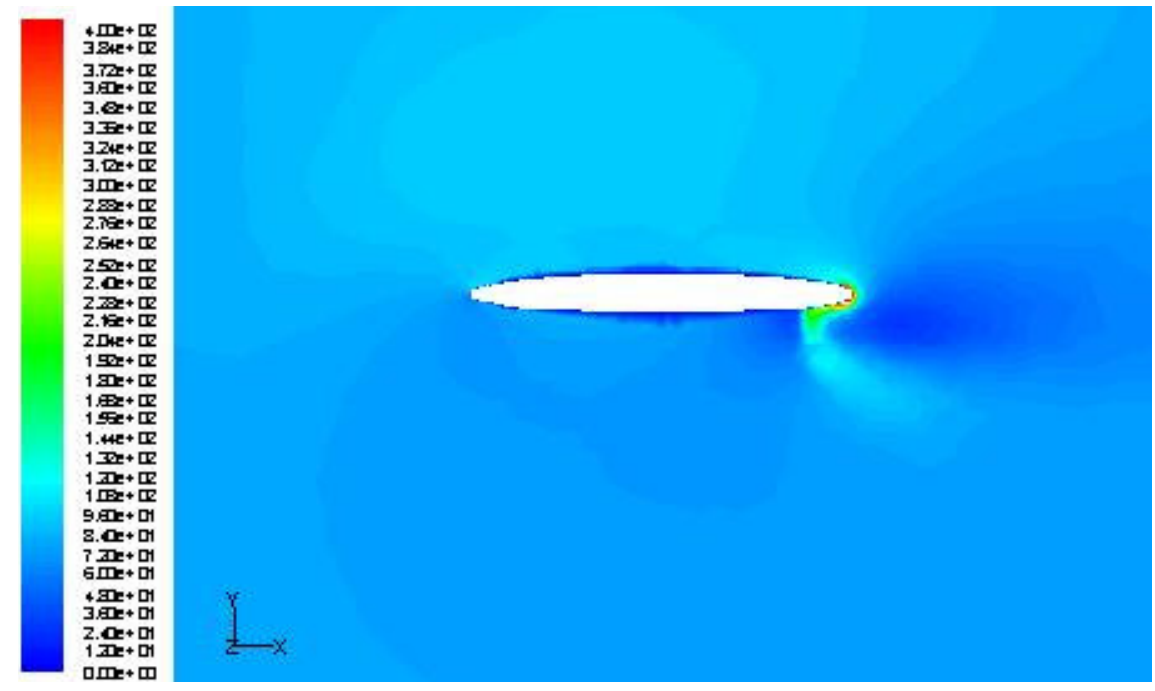

Figure 6.30: Velocity contour along centerline of 45-degree swept geometry with trailing edge blowing coefficient of 0.52 . 


\subsubsection{Span-wise Force Distribution}

By applying the $1400 \mathrm{ft} / \mathrm{s}$ trailing edge blowing across a 15 foot radius rotor blade, the computed span-wise lift distribution would be as that shown in Figure 6.31. This figure compares the lift forces, as a function of the non-dimensional radial location, $\mathrm{r} / \mathrm{R}$, for hover and the ascending blade with a flight speed of $100 \mathrm{mph}$. By varying the spanwise circulation control blowing conditions, the lift profile of the helicopter rotor can be varied, adding another design parameter to assist in optimizing the performance, noise, and vibration characteristics of the aircraft.

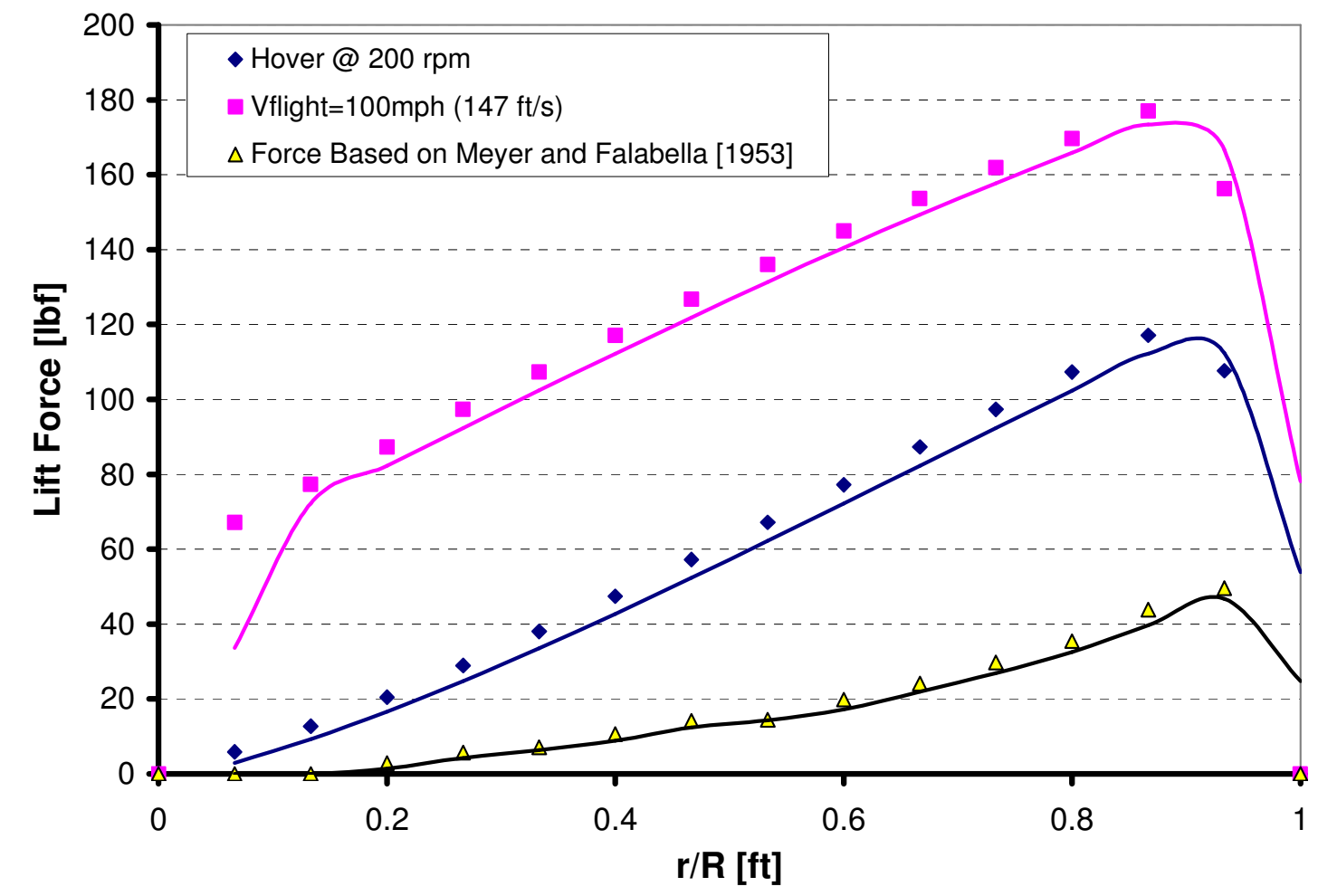

Figure 6.31: Potential lift distribution for the circulation control rotor blade, in hover and at a flight speed of $100 \mathrm{mph}$ as compared to the data from Meyer and Falabella [1953]

\subsection{Summary of Results}

Applying circulation control to the 10:1 elliptical airfoil was shown both experimentally and computationally to be able to augment the lift coefficient. The experimental analysis resulted in a change in the lift coefficient of 0.1 at 0.68 degrees angle of attack for a blowing coefficient of 0.033 , a lift augmentation, $\Delta \mathrm{C}_{1} / \mathrm{C}_{\mu}$, of 3 . At an 
angle of attack of 1.6 degrees, the lift augmentation was found to be 10 , for a lift coefficient of 0.53 at a blowing coefficient of 0.03 . The lift augmentation measured at a geometric angle of attack of 0.68 degrees represents the lift generated at a geometric angle of attack of 1.27 degrees, a change of 0.59 degrees. For the 1.6 degree case, the lift coefficient was found to be equivalent to that found at an angle of attack of 3.11 degrees, a change of 1.5 degrees.

The computational analysis for the augmentation of the lift coefficient with circulation control found a lift augmentation of 4.32 at a blowing coefficient of 0.49 , a lift augmentation of 8.6, at a zero degree angle of attack with trailing edge blowing. The combined leading and trailing edge cases found a maximum lift coefficient of 5.77 with a leading edge blowing coefficient of 0.25 and a trailing edge blowing coefficient of 0.65 . The maximum lift coefficient found with the CFD analysis, at zero degree angle of attack, is greater than the maximum lift coefficient achievable by the baseline airfoil. Thus, a representative angle of attack could not be estimated because the magnitude of the lift coefficient.

The experimental time response of the circulation control blowing slots was found to occur within $56 \mathrm{msec}$, corresponding to a rotor speed of $275 \mathrm{rpm}$, at a blowing coefficient of 0.03 . The computational model predicted a response time of $60 \mathrm{msec}$ for the full lift coefficient to be reached. The lift coefficient reaches $80 \%$ of the steady state value within $14 \mathrm{msec}(1070 \mathrm{rpm})$ which enables a change in the lift coefficient every 21 degrees at a typical rotor speed, $250 \mathrm{rpm}$. 


\section{Conclusions}

This investigation has shown that using near surface-actuated-circulation control blowing for use on rotorcraft is feasible based on the representative angle of attack change and time response characteristics, with manageable limitations on the main rotor speed. Both the experimental and computational studies showed that the forces reach their steady, augmented values within 55 to 60 milliseconds. Assuming four points for managing the circulation control lift augmentation within the blades' path, the rotor speed needs to be less than $275 \mathrm{rpm}$ well within the operating range of most helicopters.

The experimental results showed that the change in lift coefficient corresponding to an angle of attack change of \pm 1.5 degrees was reachable within the limited blowing conditions achievable with the current experimental setup. By expanding the blowing coefficients in the computational analysis, a representative angle of attack could not be determined because the predicted lift coefficient values were greater than that of the stall angle of the airfoil. This implies that circulation control can effectively simulate changes in angle of attack of the rotor blade that are comparable to current mechanically pitching of the blade with the swash-plate.

Thus, circulation control has been demonstrated to have the potential to effectively be used on a helicopter rotor that operates at a speed of up to $275 \mathrm{rpm}$. The additional lift capacity can be used to augment the performance of current helicopter rotors. Cycling the lift augmentation on a rotor operating at $275 \mathrm{rpm}$ can replace the need to physically pitch the blade with the swash-plate to overcome the asymmetry of lift. 


\section{Recommendations}

Since the use of near-surface-controlled blowing slots on the main rotor of a helicopter has been shown to be feasible from a lift augmentation standpoint several additional aspects need to be investigated with further research. Investigating the effect of circulation control blowing (leading and trailing edge) on the effective stall angle of the airfoil is recommended to further enhance the range of performance predictions of the Circulation Control Helicopter Blade.

A second recommendation is to investigate the effect of moving the leading edge blowing slot toward the mid-chord of the airfoil. The location of this blowing slot is believed to influence the performance of the airfoil at high angles of attack in delaying stall. By moving the blowing slot, the desired stall characteristics could be obtained with minimal jet momentum.

An extended study of the azimuth location effect of the Circulation Control Helicopter Blade should also be made. The sweep influence investigated to date does not incorporate reversed flow scenarios. At high flight speeds the inner portion of the descending rotor blade is moving with the freestream and can experience flow moving from back of the airfoil to front. The influence of this flight condition on the performance of the rotorcraft also needs to be understood.

After investigating the high angle of attack performance, various blowing slot, and azimuth locations, three dimensional aspects of applying circulation control to rotor blades needs to be investigated in greater detail. The first of these effects is the spanwise variation in blowing slots. The spanwise predictions made during the current research effort apply circulation control uniformly across the entire span of the rotor blade; however the characteristics can be varied across the span, creating regions without circulation control. One important parameter within the spanwise variation investigation would be how the edge of the blowing jet entrains the air moving over the unblown portion of the rotor blade.

Other three-dimensional aspects to be investigated are how twist and taper of the helicopter blade interacts with the circulation control performance. Initial estimates of the performance can be made based on the existing data, with linear approximations between 
radial positions. The approximations then would need to be compiled and verified through experimentation.

A research program should be developed to investigate these recommended areas of interest leading to the development of test rotors incorporating near-surface-actuated circulation control blowing. The logical progression of the next test specimen to be designed and tested would be a static rotor test model, followed by the fabrication and testing of a scaled, model helicopter, effectively a technology demonstrator for a circulation control rotor with surface controlled blowing slots.

Future circulation control helicopter blades models need to take precautions to ensure uniform exit velocity profiles. This can be achieved by varying the porosity of the plenum walls such that more air is permitted to pass through the porous wall near the inlet to the model. An alternative for wind tunnel models would be to have inlets on both sides (top and bottom) of the test specimen. A third method would be to add a porous wall in the span-wise direction. 


\section{References}

1. Abbott, I. H., vonDoenhoff, A. E., Theory of Wing Sections, Dover Publications Inc., New York, 1959.

2. Abramson, J., "Two-Dimensional Subsonic Wind Tunnel Evaluation of a 20Percent-Thick Circulation Control Airfoil," David W. Taylor NSRDC-ASED 311, June 1975.

3. Abramson, J., "Characteristics of a Cambered Circulation Control Airfoil Having Both Upper and Lower Surface Trailing Edge Slots," NSWCCD-50-TR2004/030, April 2004.

4. Alexander, M. G., Anders, S. G., Johnson, S. K., "Trailing Edge Circulation Control of an Airfoil at Transonic Mach Numbers," Applications of Circulation Control Technology, Ed. Ronald D. Joslin and Gregory S. Jones, Progress in Astronautics and Aeronautics Volume 214, AIAA Inc., Reston, VA, 2006, pp. 245-275.

5. Ambrosiani, J. P., “Analysis of a Circulation Controlled Elliptical Airfoil," Ph.D. Dissertation, Department of Aerospace Engineering, West Virginia University, Morgantown, WV, 1971.

6. Angle, G., O'Hara, B., Huebsch, W., Smith, J., "Experimental and Computational Investigation into the Use of the Coanda Effect on the Bell A821201," Proceedings of the 2004 NASA/ONR Circulation Control Workshop, NASA CP2005-213509, March 2005, pp.917-929

7. Barlow, J. B., Rae Jr., W. H., Pope, A., Low-Speed Wind Tunnel Testing, $3^{\text {rd }}$ Edition, John Wiley \& Sons, Inc., New York, 1999.

8. Bauman, J. L., "Development of a Control Valve to Induce an Oscillating Blowing Coefficient in a Circulation Control Rotor,' Masters Thesis, Naval Postgraduate School, Monterey, California, December 1976.

9. Cerchie, D., Halfon, E., Hammerich, A., Han, G., Taubert, L., Trouve, L., Varghese, P., Wygnanski, I., "Some Circulation and Separation Control Experiments," Applications of Circulation Control Technology, Ed. Ronald D. Joslin and Gregory S. Jones, Progress in Astronautics and Aeronautics Volume 214, AIAA Inc., Reston, VA, 2006, pp. 113-165.

10. Chang III, P. A., Slomski, J., Marino, T., Ebert, M. P., Abramson, J., "Full Reynolds-Stress Modeling of Circulation Control Airfoils," Application of Circulation Control Technology, Ed. Ronald D. Joslin and Gregory S. Jones, Progress in Astronautics and Aeronautics Volume 214, AIAA Inc., Reston, VA, 2006, pp. 445-466.

11. Chopra, I., Hong, C. H., "Flap-Lag-Torsion Aeroelastic Stability of a Circulation Control Rotor in Forward Flight," Proceedings of the 1986 Circulation Control Workshop, NASA CP-2432, February 1986, pp.315-352.

12. Churchill, R. A., "Coanda Jet Around a Cylinder with an Interacting Adjacent Surface," Ph.D. Dissertation, Department of Mechanical and Aerospace Engineering, West Virginia University, Morgantown, WV, 1992.

13. Dash, S. M., York, B. J., Sinha, N., Dvorak, F. A., "Wall Jet Analysis for Circulation Control Aerodynamics, Part I: Fundamental CFD and Turbulence 
Modeling Concepts," Proceedings of the 1986 Circulation Control Workshop, NASA CP-2432, February 1986, pp.23-69.

14. Debus, A. G. (Editor), Marquis - Who's Who, Inc., World Who's Who in Science (From Antiquity to the Present), First Edition, 1968, p. 348.

15. Durand, W. F. (Editor), Aerodynamic Theory, A General Review of Progress Volume II: General Aerodynamic Theory, Perfect Fluids, Peter Smith Publisher, Inc., Gloucester, Massachusetts, 1976.

16. Dvorak, F. A., Dash, S. M., "Wall Jet Analysis for Circulation Control Aerodynamics, Part II: Zonal Modeling Concepts for Wall Jet/Potential Flow Coupling," Proceedings of the 1986 Circulation Control Workshop, NASA CP2432, February 1986, pp. 165-181.

17. Englar, R. J., "Experimental Investigation of the High Velocity Coanda Wall Jet Applied to Bluff Trailing Edge Circulation Control Airfoils," Naval Research and Development Center Report 4708, September 1975.

18. Englar, R. J., Smith, M. J., Niebur, C. S., Gregory, S. D., "Development of Pneumatic Aerodynamic Concepts for Control of Lift, Drag and Moments plus Lateral/Directional Stability of Automotive Vehicles," SAE Technical Paper 960673, February 1996.

19. Englar, R. J., "Overview of Circulation Control Pneumatic Aerodynamics: Blown Force ad Moment Augmentation and Modification as Applied Primarily to FixedWing Aircraft," Proceedings of the 2004 NASA/ONR Circulation Control Workshop, NASA CP-2005-213509, March 2005, pp. 37-79.

20. Franke, M. E., Harvell, J. K., "Wind Tunnel Studies of Circulation Control Elliptical Airfoils," Proceedings of the 1986 Circulation Control Workshop, NASA CP-2432, February 1986, pp. 267-287.

21. Friedman, C., Arieli, R., Levy, Y., "Numerical Investigation of Unsteady Circulation Control," AIAA Technical Paper Series, Paper No. 2007-3918, June 2007.

22. Gibbs, E. H., "Analysis of Circulation Controlled Airfoils," Ph.D. Dissertation, Department of Aerospace Engineering, West Virginia University, Morgantown, WV, 1975.

23. Harness, G. S., "An Experimental Investigation of a Circulation Controlled Cambered Elliptical Airfoil," Masters Thesis, Department of Aerospace Engineering, West Virginia University, Morgantown, WV, 1970.

24. Imber, R., Rogers, E., Abramson, J., "Exploratory Investigations of Circulation Control Technology: Overview for Period 1987-2003 at NSWCCD," Proceedings of the 2004 NASA/ONR Circulation Control Workshop, NASA CP-2005-213509, March 2005, pp. 569-594.

25. Johnson, J. S., "An Experimental Investigation of Two Dimensional Curved Jets," Masters Thesis, Department of Aerospace Engineering, West Virginia University, Morgantown, WV, 1975.

26. Keener, E. R., Sanderfer, D. T., Wood, N. J., "Pressure Distributions and OilFlow Patterns for a Swept Circulation Control Wing," Proceedings of the 1986 Circulation Control Workshop, NASA CP-2432, February 1986, pp. 209-238. 
27. Kind, R. J., "A Calculation Method for Circulation Control by Tangential Blowing Around a Bluff Trailing Edge," Aeronautical Quarterly, Vol. XIX., August 1968, pp. 205-223

28. Kind, R. J., Maull, D. J., "An Experimental Investigation of a Low-Speed Circulation-Controlled Aerofoil," The Aeronautical Quarterly, Vol. XIX, May 1968, pp. 170-182.

29. Kumagai, H., "Parametric Analysis of a Passive Cyclic Control Device for Helicopters," NASA Contractor Report 166608, October 1984.

30. Loth, J. L., Chandra, S., Gibbs E. H., "Inlet and Internal Aerdynamics of a VTOL Thrust Augmenter," Final Report, Office of Naval Research Contract N00014-75C-0324, January 1976.

31. McLachlan, B. G., "On the Effect of Leading Edge Blowing on Circulation Control Airfoil Aerodynamics," Proceedings of the 1986 Circulation Control Workshop, NASA CP-2432, February 1986, pp. 199-208.

32. Meyer, J. R. Jr, Falabella, G. Jr., "An Investigation of the Experimental Aerodynamic Loading on a Model Helicopter Rotor Blade," NACA Technical Note 2953, May 1953.

33. Myer, D. P., "An Experimental Investigation of a Circulation Controlled Cambered Elliptical Airfoil with a Rounded Trailing Edge," Masters Thesis, Department of Aerospace Engineering, West Virginia University, Morgantown, WV, 1972.

34. Newman, B. G., "The Deflexion of Plane Jets by Adjacent Boundaries - Coanda Effect," Boundary Layer and Flow Control, Pergamon Press Vol. 1, 1961, p 232.

35. Novak, C. J., Cornelius, K. C., "Investigations of a Circulation Control Airfoil Flowfield using an Advanced Laser Velocimeter," Proceedings of the 1986 Circulation Control Workshop, NASA CP-2432, February 1986, pp. 71-88.

36. Owen, K. F., "Measurement and Analysis of Circulation Control Airfoils," Proceedings of the 2004 NASA/ONR Circulation Control Workshop, NASA CP2005-213509, March 2005, pp.975-983.

37. Paterson, E. G., Baker, W. J., "RANS and Detached-Eddy Simulation of the NCCR Airfoil," Application of Circulation Control Technology, Ed. Ronald D. Joslin and Gregory S. Jones, Progress in Astronautics and Aeronautics Volume 214, AIAA Inc., Reston, VA, 2006, pp. 421-444.

38. Pulliam, T. H., Jespersen, D. C., Barth, T. J., "Navier-Stokes Computations for Circulation Control Airfoils," Proceedings of the 1986 Circulation Control Workshop, NASA CP-2432, February 1986, pp. 135-163.

39. Ramaprian, B. R., Shivaprasad, B. G., "Mean Flow Measurements in Turbulent Boundary Layers Along Mildly Curved Surfaces," AIAA Journal Vol. 15 No. 2, February 1977, pp. 189 - 196.

40. Ramaprian, B. R., Shivaprasad, B. G., "Turbulent Measurements in Boundary Layers Along Mildly Curved Surfaces," Journal of Fluids Engineering Transactions of ASME Vol. 100 No. 1, March 1978, pp. 37 - 46.

41. Reader, K. R., "A Control System for the Wind Tunnel Model of a Reverse Blowing Circulation Control Rotor (RC-CCR)," David W. Taylor Naval Ship Research and Development Center Report 76-0062, April 1976. 
42. Reader, K. R., Wilkerson, J. B., "Circulation Control Applied to a High Speed Helicopter Rotor," David W. Taylor Naval Ship Research and Development Center Report 77-0024, February 1977.

43. Roberts, L., "A Theory for Turbulent Curved Wall Jets," Proceedings of the 1986 Circulation Control Workshop, NASA CP-2432, February 1986, pp. 99-112.

44. Shrewsbury, G. D., "Evaluation of a Research Circulation Control Airfoil using Navier-Stokes Methods," Proceedings of the 1986 Circulation Control Workshop, NASA CP-2432, February 1986, pp. 115-134.

45. Spaid, F. W., Keener, E. R., "Boundary-Layer and Wake Measurements on a Swept, Circulation-Control Wing," Proceedings of the 1986 Circulation Control Workshop, NASA CP-2432, February 1986, pp. 239-266.

46. Smith, J. E., Loth, J. L., Craven, R. P. M., Bond, R., "Piezoelectric Actuators for Circulation Controlled Rotorcraft" US Patent 6,425,553B1, July 2002.

47. Stratford, B. S., "The Prediction of Separation of the Turbulent Boundary Layer," Journal of Fluid Mechanics, Vol. 5, Cambridge University Press, 1959, pp. 1-16.

48. Theodorsen, T., "Theory of Wing Sections of Arbitrary Shape," NACA-TR-411, 1931.

49. Theodorsen, T., Garrick, L. E., "General Potential Theory of Arbitrary Wing Sections," NACA TR-452, 1940.

50. Viegas, J. R., Rubesin, M. W., MacCormack, R. W., “Navier-Stokes Calculations and Turbulence Modeling in the Trailing Edge Region of a Circulation Control Airfoil," Proceedings of the 1986 Circulation Control Workshop, NASA CP2432, February 1986, pp.1-22.

51. White, F. M., Viscous Fluid Flow Second Edition, McGraw Hill, Inc., New York, 1991.

52. Wilson, D. L., Goldstein, R. J., "Turbulent Wall Jets with Cylindrical Streamwise Surface Curvature," Journal of Fluids Engineering - ASME Transactions Vol. 98 no. 3, September 1976, pp. 550-557.

53. Wilson, J. D., “Thrust Augmented Wing Sections in Potential Flow," Ph.D. Dissertation, Department of Aerospace Engineering, West Virginia University, Morgantown, WV, 1973.

54. Wood, N. J., "The Further Development of Circulation Control Airfoils," Proceedings of the 1986 Circulation Control Workshop, NASA CP-2432, February 1986, pp.183-196.

55. Young, A. D., Boundary Layers, AIAA Education Series, American Institute of Aeronautics and Astronautics, Inc., Washington, DC, 1989.

56. "Air - Temperature, Pressure and Density", The Engineering Toolbox, 2007. 1 Sept $2007<$ http://www.engineeringtoolbox.com/air-temperature-pressuredensity-d_771.html> 


\section{Appendix}

A

Test Section Blockage Data 
Table A. 1: Summary of the blockage ratios determined for the NACA 0009 and NACA 0012 airfoils with a chordlength of 6 inches

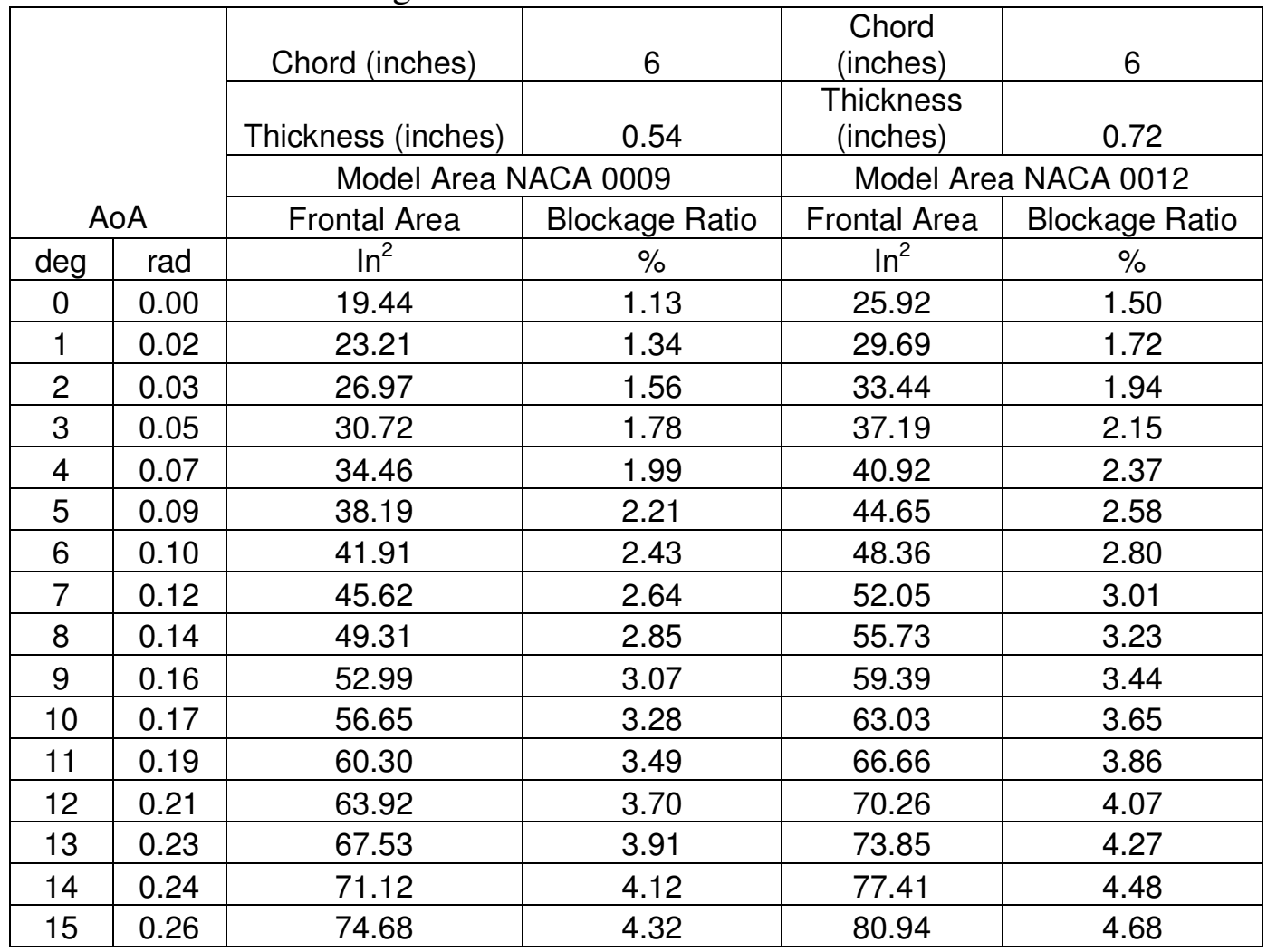


Table A. 2: Summary of the blockage ratios determined for the NACA 0009 and NACA 0012 airfoils with a chordlength of 9 inches

\begin{tabular}{|c|c|c|c|c|c|}
\hline & & \multicolumn{2}{|c|}{ NACA 0009 Airfoil } & \multicolumn{2}{|c|}{ NACA 0012 Airfoil } \\
\hline & & Chord (inches) & 9 & Chord (inches) & 9 \\
\hline & & $\begin{array}{l}\text { Thickness } \\
\text { (inches) }\end{array}$ & 0.81 & $\begin{array}{c}\text { Thickness } \\
\text { (inches) }\end{array}$ & 1.08 \\
\hline & & \multicolumn{2}{|c|}{ Model Area NACA 0009} & \multicolumn{2}{|c|}{ Model Area NACA 0012} \\
\hline \multicolumn{2}{|c|}{ AoA } & Frontal Area & $\begin{array}{c}\text { Blockage } \\
\text { Ratio }\end{array}$ & Frontal Area & $\begin{array}{c}\text { Blockage } \\
\text { Ratio }\end{array}$ \\
\hline deg & $\mathrm{rad}$ & $\ln ^{2}$ & $\%$ & $\ln ^{2}$ & $\%$ \\
\hline 0 & 0.00 & 29.16 & 1.69 & 38.88 & 2.25 \\
\hline 1 & 0.02 & 34.81 & 2.01 & 44.53 & 2.58 \\
\hline 2 & 0.03 & 40.45 & 2.34 & 50.16 & 2.90 \\
\hline 3 & 0.05 & 46.08 & 2.67 & 55.78 & 3.23 \\
\hline 4 & 0.07 & 51.69 & 2.99 & 61.39 & 3.55 \\
\hline 5 & 0.09 & 57.29 & 3.32 & 66.97 & 3.88 \\
\hline 6 & 0.10 & 62.87 & 3.64 & 72.53 & 4.20 \\
\hline 7 & 0.12 & 68.43 & 3.96 & 78.08 & 4.52 \\
\hline 8 & 0.14 & 73.97 & 4.28 & 83.59 & 4.84 \\
\hline 9 & 0.16 & 79.49 & 4.60 & 89.09 & 5.16 \\
\hline 10 & 0.17 & 84.98 & 4.92 & 94.55 & 5.47 \\
\hline 11 & 0.19 & 90.45 & 5.23 & 99.99 & 5.79 \\
\hline 12 & 0.21 & 95.89 & 5.55 & 105.39 & 6.10 \\
\hline 13 & 0.23 & 101.30 & 5.86 & 110.77 & 6.41 \\
\hline 14 & 0.24 & 106.68 & 6.17 & 116.11 & 6.72 \\
\hline 15 & 0.26 & 112.02 & 6.48 & 121.41 & 7.03 \\
\hline
\end{tabular}


Table A. 3: Summary of the blockage ratios for 20 inch and 10 inch chordlength 10:1 elliptical airfoils

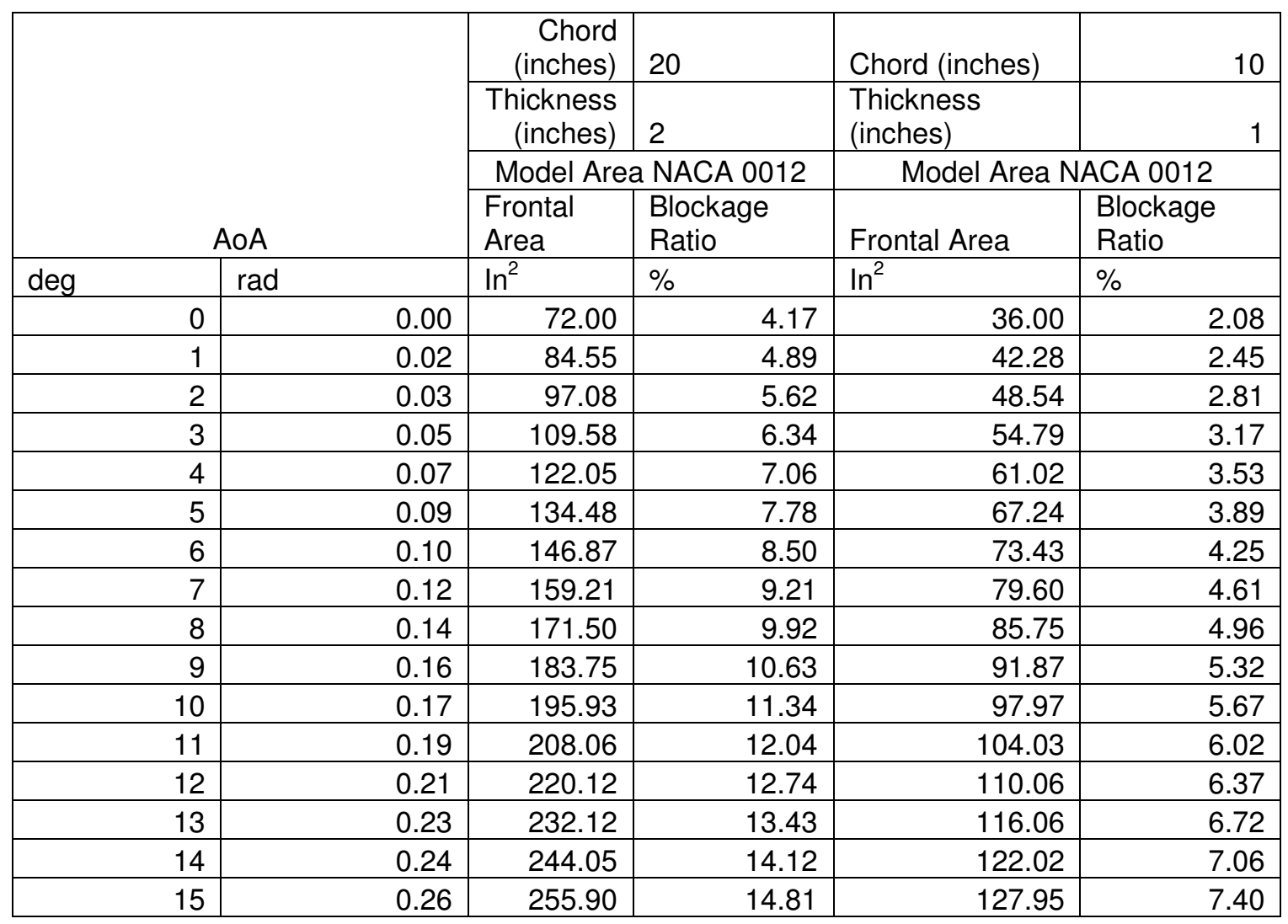




\section{Appendix}

B

Reynolds Number Data 
Table B. 1: Summary of the rotational velocity and local Reynolds number for a $15 \mathrm{ft}$ radius rotor blade

\begin{tabular}{|c|c|c|c|c|c|c|}
\hline \multirow{2}{*}{$\begin{array}{c}\text { Blade } \\
\text { Station }\end{array}$} & \multicolumn{2}{|c|}{ Hover @ 200 rpm } & \multicolumn{2}{c|}{$\mathrm{V}_{\text {flight }}=50 \mathrm{mph}(73 \mathrm{ft} / \mathrm{s})$} & \multicolumn{2}{c|}{$\mathrm{V}_{\text {flight }}=100 \mathrm{mph}(147 \mathrm{ft} / \mathrm{s})$} \\
\hline & $\mathrm{V}_{\omega}$ & $\mathrm{RE}$ & $\mathrm{V}_{\text {relative }}$ & $\mathrm{RE}$ & $\mathrm{V}_{\text {relative }}$ & $\mathrm{RE}$ \\
\hline $\mathrm{ft}$ & $\mathrm{ft} / \mathrm{s}$ & - & $\mathrm{ft} / \mathrm{s}$ & - & $\mathrm{ft} / \mathrm{s}$ & - \\
\hline 1 & 20.9 & $1.44 \mathrm{E}+05$ & 94.3 & $6.50 \mathrm{E}+05$ & 167.6 & $1.15 \mathrm{E}+06$ \\
\hline 2 & 41.9 & $2.89 \mathrm{E}+05$ & 115.2 & $7.94 \mathrm{E}+05$ & 188.6 & $1.30 \mathrm{E}+06$ \\
\hline 3 & 62.8 & $4.33 \mathrm{E}+05$ & 136.2 & $9.38 \mathrm{E}+05$ & 209.5 & $1.44 \mathrm{E}+06$ \\
\hline 4 & 83.8 & $5.77 \mathrm{E}+05$ & 157.1 & $1.08 \mathrm{E}+06$ & 230.4 & $1.59 \mathrm{E}+06$ \\
\hline 5 & 104.7 & $7.21 \mathrm{E}+05$ & 178.1 & $1.23 \mathrm{E}+06$ & 251.4 & $1.73 \mathrm{E}+06$ \\
\hline 6 & 125.7 & $8.66 \mathrm{E}+05$ & 199.0 & $1.37 \mathrm{E}+06$ & 272.3 & $1.88 \mathrm{E}+06$ \\
\hline 7 & 146.6 & $1.01 \mathrm{E}+06$ & 219.9 & $1.52 \mathrm{E}+06$ & 293.3 & $2.02 \mathrm{E}+06$ \\
\hline 8 & 167.6 & $1.15 \mathrm{E}+06$ & 240.9 & $1.66 \mathrm{E}+06$ & 314.2 & $2.16 \mathrm{E}+06$ \\
\hline 9 & 188.5 & $1.30 \mathrm{E}+06$ & 261.8 & $1.80 \mathrm{E}+06$ & 335.2 & $2.31 \mathrm{E}+06$ \\
\hline 10 & 209.4 & $1.44 \mathrm{E}+06$ & 282.8 & $1.95 \mathrm{E}+06$ & 356.1 & $2.45 \mathrm{E}+06$ \\
\hline 11 & 230.4 & $1.59 \mathrm{E}+06$ & 303.7 & $2.09 \mathrm{E}+06$ & 377.1 & $2.60 \mathrm{E}+06$ \\
\hline 12 & 251.3 & $1.73 \mathrm{E}+06$ & 324.7 & $2.24 \mathrm{E}+06$ & 398.0 & $2.74 \mathrm{E}+06$ \\
\hline 13 & 272.3 & $1.88 \mathrm{E}+06$ & 345.6 & $2.38 \mathrm{E}+06$ & 418.9 & $2.89 \mathrm{E}+06$ \\
\hline 14 & 293.2 & $2.02 \mathrm{E}+06$ & 366.5 & $2.53 \mathrm{E}+06$ & 439.9 & $3.03 \mathrm{E}+06$ \\
\hline 15 & 314.2 & $2.16 \mathrm{E}+06$ & 387.5 & $2.67 \mathrm{E}+06$ & 460.8 & $3.17 \mathrm{E}+06$ \\
\hline \multicolumn{7}{|r}{}
\end{tabular}




\section{Appendix}

C

\section{Data Reduction Programs}




\section{Program C-1: For Data Files Without Pressure Data}

oThis code was written by Gerald M. Angle II, Center for Industrial \%Research Applications, Mechanical and Aerospace Engineering, in \%February 2008 and utilized Matlab Release $2007 a$ oClear Variables and Command Window

clear

clc

oSpecify Files to be summarized

data $0=$ importdata ('D: \Bears \dissertation\wind_tunnel_data \apr1_2008\Acti ve \Solenoid_closingTElower_Vpt6_TEp30_run1-P0.txt');

$[\mathrm{M}, \mathrm{N}]=$ size (data0.data);

oInitialize Variables

count $=1 ; \operatorname{TLV}=\operatorname{zeros}(\mathrm{M}, 1) ; \mathrm{TL}=\operatorname{zeros}(\mathrm{M}, 1) ; \operatorname{TDV}=\operatorname{zeros}(\mathrm{M}, 1) ; \mathrm{TD}=\operatorname{zeros}(\mathrm{M}, 1)$;

$\operatorname{BLV}=\operatorname{zeros}(\mathrm{M}, 1)$; $\mathrm{BL}=\operatorname{zeros}(\mathrm{M}, 1)$; $\mathrm{BDV}=\operatorname{zeros}(\mathrm{M}, 1)$; $\mathrm{BD}=\operatorname{zeros}(\mathrm{M}, 1) ; \mathrm{x}=\operatorname{zeros}(\mathrm{M}, 1)$;

osplit data matrix into arrays for TL, TD, BL, BD, and P0-31

for count $=1: \mathrm{M}$

TLV (count, 1$)=\operatorname{data} 0 . \operatorname{data}($ count, 2$)$; TL ( count, 1$)=\operatorname{data} 0$. data $($ count, 3$)$; BLV (co unt, 1$)=$ data 0. data $($ count, 4$)$;

BL ( count, 1 ) =data 0 . data ( count, 5); TDV (count, 1) =data 0 .data (count, 6) ; TD (cou $\mathrm{nt}, 1)=$ data 0 . data $($ count, 7$)$;

BDV $($ count, 1$)=$ data 0. data $($ count, 8$) ; B D($ count, 1$)=$ data 0. data $($ count, 9$) ; \times($ coun

$t, 1)=$ count

end

oCalculate Total Lift and Drag and statistics

av_TL $=(0.005 *$ mean $(T D V)+0.3972) *$ mean $(T L V)$;

av_TD $=(-0.0083 *$ mean $(\mathrm{TLV})+0.5716) *$ mean $(\mathrm{TDV})$;

av_TD2 $=(-0.0083 *$ mean $($ TLV $)+0.5716) *$ mean $(T D V)$;

av_BL $=(0.0019 *$ mean $(B D V)+0.7163) *$ mean $(B L V)$;

av_BD $=(-0.0012 *$ mean $(B L V)+0.587) *$ mean $(B D V)$;

TotalL=TLV+BLV;

TotalD=TDV+BDV;

av_totalL=mean ( TotalL);

av_totalD=mean (TotalD);

std_dev_TL=std (TLV);

std_dev_TD=std (TDV);

std_dev_BL=std (BLV);

std_dev_BD=std (BDV);

std_dev_totalL=std ( TotalL);

std_dev_totalD=std (TotalD);

$\because \mathrm{Plots}$

[M2, N2 ] =size (TL);

$\mathrm{x} \_$chord=1:1:M2;

plot (x_chord, TL); title('Top Lift

Datapoints'); xlabel('Datapoint'); ylabel('Top Lift Force (lbf)');

figure

plot (x_chord,BL); title('Bottom Lift

Datapoints'); xlabel('Datapoint'); ylabel('Bottom Lift Force (lbf)');

figure

plot (x_chord, TD); title('Top Drag

Datapoints'); xlabel('Datapoint');ylabel('Top Drag Force (lbf)');

figure

plot(x_chord, BD); title('Bottom Drag

Datapoints'); xlabel('Datapoint'); ylabel('Bottom Drag Force (lbf)');

oPrint summary of statistics to screen 
fprintf('Property \t Average Value \t Average Standard Deviation \t Units $\left.\backslash n^{\prime}\right)$;

fprintf('Top Lift \t of \t $\frac{0}{\partial} \backslash t$ lbs $\backslash n '$, av_TL, std_dev_TL)

fprintf('Top Drag \t $\frac{\partial}{t} \backslash t \frac{\circ}{t} \backslash t$ lbs $\backslash n^{\prime}$, av_TD, std_dev_TD)

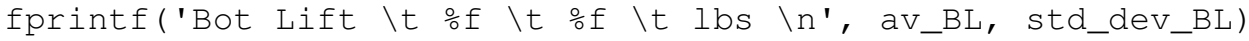

fprintf('Bot Drag \t $\frac{\mathrm{f}}{t} \backslash \mathrm{t} \% \mathrm{f} \backslash t \mathrm{lbs} \backslash \mathrm{n}^{\prime}, \mathrm{av}$ _BD, std_dev_BD)

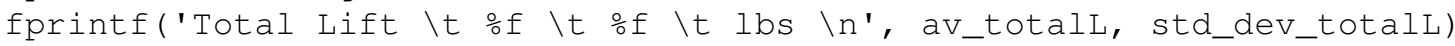

fprintf('Total Drag $\backslash t \circ f \backslash t \circ f \backslash t$ lbs $\backslash n^{\prime}$, av_totald, std_dev_totald) 


\section{Program C-2: Data File With Pressure Data}

oThis code was written by Gerald M. Angle II, Center for Industrial oResearch Applications, Mechanical and Aerospace Engineering, in \%February 2008 and utilized Matlab Release $2007 \mathrm{a}$

oclear variables and screen

clear

Clc

ospecify Files to be summarized

data $0=$ importdata ('D: \Bears \dissertation \wind_tunnel_data \cals $\backslash$ LC_cal3_4 -08\BDneg0-P0.txt');

datal=importdata( 'D: \Bears \dissertation \wind_tunnel_data \cals $\backslash$ LC_cal3_4 -08\BDneg0-P1.txt') ;

data2=importdata ( 'D: \Bears \dissertation\wind_tunnel_data \cals \LC_cal3_4 -08\BDneg0-P2.txt');

data3=importdata(' D: \Bears \dissertation \wind_tunnel_data \Cals $\backslash$ LC_cal3_4 -08\BDneg0-P3.txt'):

data 4=importdata(' D: \Bears \dissertation \wind_tunnel_data \cals $\backslash$ LC_cal3_4 -08\BDneg0-P4.txt') ;

data $5=$ importdata ( 'D: \Bears \dissertation\wind_tunnel_data \cals \LC_cal3_4 _08\BDneg0-P5.txt');

data6=importdata ('D: \Bears \dissertation\wind_tunnel_data \Cals \LC_cal3_4 -08\BDneg0-P6.txt');

data 7=importdata('D: \Bears \dissertation\wind_tunnel_data \cals \LC_cal3_4 -08\BDneg0-P7.txt');

data $8=$ importdata ('D: \Bears \dissertation \wind_tunnel_data \cals $\backslash$ LC_cal3_4 -08\BDneg0-P8.txt');

data9=importdata(' D: \Bears \dissertation \wind_tunnel_data \cals $\backslash$ LC_cal3_4 -08\BDneg0-P9.txt');

data10=importdata('D: \Bears \dissertation\wind_tunnel_data \Cals LC_Cal3_ 4_08\BDneg0-P10.txt' ) ;

data11=importdata ('D: \Bears \dissertation \wind_tunnel_data \cals \LC_cal3_ 4_08\BDneg0-P11.txt') ;

data12=importdata ('D: \Bears \dissertation \wind_tunnel_data \cals \LC_cal3_ 4_08\BDneg0-P12.txt') ;

data13=importdata ('D: \Bears \dissertation \wind_tunnel_data \cals $\backslash$ LC_Cal3_ 4_08\BDneg0-P13.txt');

data14=importdata('D: \Bears \dissertation \wind_tunnel_data \cals $\backslash$ LC_Cal3_ 4_08\BDneg0-P14.txt') ;

data15=importdata('D: \Bears \dissertation \wind_tunnel_data \Cals LC_Cal3_ 4_08\BDneg0-P15.txt');

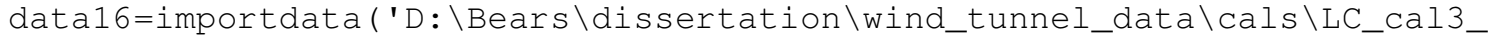
4_08\BDneg0-P16.txt');

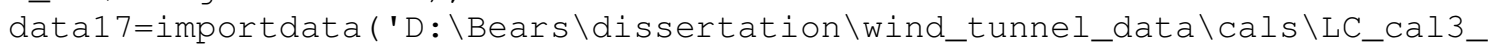
4_08\BDneg0-P17.txt') ;

data18=importdata('D: \Bears \dissertation\wind_tunnel_data \Cals $\backslash$ LC_Cal3_ 4_08\BDneg0-P18.txt') ;

data19=importdata ('D: \Bears \dissertation \wind_tunnel_data \cals \LC_cal3_ 4_08\BDneg0-P19.txt');

data20=importdata('D: \Bears \dissertation \wind_tunnel_data \cals $\backslash$ LC_Cal3_ 4_08\BDneg0-P20.txt') ;

data21=importdata ('D: \Bears \dissertation\wind_tunnel_data \cals $\backslash$ LC_Cal3_ 4_08\BDneg0-P21.txt');

data22=importdata ( 'D: \Bears \dissertation \wind_tunnel_data \cals $\backslash$ LC_cal3_ 4_08\BDneg0-P22.txt') ; 
data23=importdata ('D: \Bears \dissertation \wind_tunnel_data \cals \LC_cal3_ 4_08\BDneg0-P23.txt') ;

data24=importdata ('D: \Bears \dissertation \wind_tunnel_data \cals \LC_cal3_ 4_08\BDneg0-P24.txt');

data $25=$ importdata ( 'D: \Bears \dissertation \wind_tunnel_data \cals $\backslash$ LC_Cal3_ 4_08\BDneg0-P25.txt');

data26=importdata ('D: \Bears \dissertation \wind_tunnel_data \cals \LC_cal3_ 4_08\BDneg0-P26.txt');

data27=importdata ('D: \Bears \dissertation \wind_tunnel_data \cals \LC_cal3_ 4_08\BDneg0-P27.txt');

data28=importdata ('D: \Bears \dissertation \wind_tunnel_data \cals \LC_cal3_ 4_08\BDneg0-P28.txt');

data29=importdata ('D: \Bears \dissertation \wind_tunnel_data \cals \LC_cal3_ 4_08\BDneg0-P29.txt') ;

data30=importdata ('D: \Bears \dissertation \wind_tunnel_data \cals $\backslash$ LC_Cal3_ 4_08\BDneg0-P30.txt');

data31=importdata ('D: \Bears \dissertation \wind_tunnel_data \cals $\backslash$ LC_Cal3_ 4_08\BDneg0-P31.txt');

$[\mathrm{M}, \mathrm{N}]=\mathrm{size}($ data 0. data)

onitialize Variables

count $=1 ; \operatorname{TLVO}=\operatorname{zeros}(\mathrm{M}, 1) ; \mathrm{TL} 0=\operatorname{zeros}(\mathrm{M}, 1) ; \operatorname{TDV} 0=\operatorname{zeros}(\mathrm{M}, 1) ; \operatorname{TD} 0=\operatorname{zerOs}(\mathrm{M}, 1)$;

$\mathrm{BLV} 0=\operatorname{zeros}(\mathrm{M}, 1) ; \mathrm{BL} 0=\operatorname{zeros}(\mathrm{M}, 1) ; \mathrm{BDV} 0=\operatorname{zeros}(\mathrm{M}, 1) ; \mathrm{BD} 0=\operatorname{zeros}(\mathrm{M}, 1) ; \mathrm{PV} 0=\mathrm{zeros}$

$(\mathrm{M}, 1) ; \mathrm{PO}=\operatorname{zeros}(\mathrm{M}, 1)$;

$\mathrm{TLV} 1=\operatorname{zeros}(\mathrm{M}, 1) ; \mathrm{TL} 1=\operatorname{zeros}(\mathrm{M}, 1) ; \operatorname{TDV} 1=\operatorname{zeros}(\mathrm{M}, 1) ; \operatorname{TD} 1=\operatorname{zeros}(\mathrm{M}, 1) ; \mathrm{BLV} 1=\operatorname{zero}$ $\mathrm{S}(\mathrm{M}, 1)$;

$\mathrm{BL} 1=\operatorname{zeros}(\mathrm{M}, 1) ; \mathrm{BDV} 1=\operatorname{zeros}(\mathrm{M}, 1) ; \mathrm{BD} 1=\operatorname{zeros}(\mathrm{M}, 1) ; \mathrm{PV} 1=\operatorname{zeros}(\mathrm{M}, 1) ; \mathrm{P} 1=\operatorname{zeros}(\mathrm{M}$ , 1 ) ;

$\mathrm{TLV} 2=\operatorname{zeros}(\mathrm{M}, 1) ; \mathrm{TL} 2=\operatorname{zeros}(\mathrm{M}, 1) ; \operatorname{TDV} 2=\operatorname{zeros}(\mathrm{M}, 1) ; \operatorname{TD} 2=\operatorname{zeros}(\mathrm{M}, 1) ; \mathrm{BLV} 2=\operatorname{zero}$ $\mathrm{S}(\mathrm{M}, 1)$;

$\mathrm{BL} 2=\operatorname{zeros}(\mathrm{M}, 1) ; \mathrm{BDV} 2=\operatorname{zeros}(\mathrm{M}, 1) ; \mathrm{BD} 2=\operatorname{zeros}(\mathrm{M}, 1) ; \mathrm{PV} 2=\operatorname{zeros}(\mathrm{M}, 1) ; \mathrm{P} 2=\operatorname{zeros}(\mathrm{M}$ , 1) ;

$\operatorname{TLV} 3=\operatorname{zeros}(\mathrm{M}, 1) ; \mathrm{TL} 3=\operatorname{zeros}(\mathrm{M}, 1) ; \operatorname{TDV} 3=\operatorname{zeros}(\mathrm{M}, 1) ; \operatorname{TD} 3=\operatorname{zeros}(\mathrm{M}, 1) ; \mathrm{BLV} 3=\operatorname{zero}$ $\mathrm{S}(\mathrm{M}, 1)$;

$\mathrm{BL} 3=\operatorname{zeros}(\mathrm{M}, 1) ; \mathrm{BDV} 3=\operatorname{zeros}(\mathrm{M}, 1) ; \mathrm{BD} 3=\operatorname{zeros}(\mathrm{M}, 1) ; \mathrm{PV} 3=\operatorname{zeros}(\mathrm{M}, 1) ; \mathrm{P} 3=\operatorname{zeros}(\mathrm{M}$ , 1 ) ;

$\operatorname{TLV} 4=\operatorname{zeros}(\mathrm{M}, 1) ; \mathrm{TL} 4=\operatorname{zeros}(\mathrm{M}, 1) ; \operatorname{TDV} 4=\operatorname{zeros}(\mathrm{M}, 1) ; \operatorname{TD} 4=\operatorname{zeros}(\mathrm{M}, 1) ; \mathrm{BLV} 4=\mathrm{zerO}$ $\mathrm{S}(\mathrm{M}, 1)$;

$\mathrm{BL} 4=\operatorname{zeros}(\mathrm{M}, 1) ; \operatorname{BDV} 4=\operatorname{zeros}(\mathrm{M}, 1) ; \mathrm{BD} 4=\operatorname{zeros}(\mathrm{M}, 1) ; \mathrm{PV} 4=\operatorname{zeros}(\mathrm{M}, 1) ; \mathrm{P} 4=\operatorname{zeros}(\mathrm{M}$ , 1 ) ;

$\operatorname{TLV} 5=\operatorname{zeros}(\mathrm{M}, 1) ; \mathrm{TL} 5=\operatorname{zeros}(\mathrm{M}, 1) ; \operatorname{TDV} 5=\operatorname{zeros}(\mathrm{M}, 1) ; \operatorname{TD} 5=\operatorname{zeros}(\mathrm{M}, 1) ; \mathrm{BLV} 5=\mathrm{zero}$ $\mathrm{S}(\mathrm{M}, 1)$;

$\mathrm{BL} 5=\operatorname{zeros}(\mathrm{M}, 1) ; \mathrm{BDV} 5=\operatorname{zeros}(\mathrm{M}, 1) ; \mathrm{BD} 5=\operatorname{zeros}(\mathrm{M}, 1) ; \mathrm{PV} 5=\operatorname{zeros}(\mathrm{M}, 1) ; \mathrm{P} 5=\operatorname{zeros}(\mathrm{M}$ , 1$)$;

$\operatorname{TLV} 6=\operatorname{zeros}(\mathrm{M}, 1) ; \operatorname{TL} 6=\operatorname{zeros}(\mathrm{M}, 1) ; \operatorname{TDV} 6=\operatorname{zeros}(\mathrm{M}, 1) ; \operatorname{TD} 6=\operatorname{zeros}(\mathrm{M}, 1) ; \mathrm{BLV} 6=\operatorname{zero}$ $\mathrm{S}(\mathrm{M}, 1)$;

$\mathrm{BL} 6=\operatorname{zeros}(\mathrm{M}, 1) ; \mathrm{BDV} 6=\operatorname{zeros}(\mathrm{M}, 1) ; \mathrm{BD} 6=\operatorname{zeros}(\mathrm{M}, 1) ; \mathrm{PV} 6=\operatorname{zeros}(\mathrm{M}, 1) ; \mathrm{P} 6=\operatorname{zeros}(\mathrm{M}$ , 1$)$;

$\operatorname{TLV} 7=\operatorname{zeros}(\mathrm{M}, 1) ; \operatorname{TL} 7=\operatorname{zeros}(\mathrm{M}, 1) ; \operatorname{TDV} 7=\operatorname{zeros}(\mathrm{M}, 1) ; \operatorname{TD} 7=\operatorname{zeros}(\mathrm{M}, 1) ; \operatorname{BLV} 7=\operatorname{zero}$ $\mathrm{S}(\mathrm{M}, 1)$;

$\operatorname{BL} 7=\operatorname{zeros}(M, 1) ; \operatorname{BDV} 7=\operatorname{zeros}(M, 1) ; \operatorname{BD} 7=\operatorname{zeros}(M, 1) ; \operatorname{PV} 7=\operatorname{zeros}(M, 1) ; P 7=\operatorname{zeros}(M$ , 1 ) ;

$\operatorname{TLV} 8=\operatorname{zeros}(\mathrm{M}, 1) ; \mathrm{TL} 8=\operatorname{zeros}(\mathrm{M}, 1) ; \operatorname{TDV} 8=\operatorname{zeros}(\mathrm{M}, 1) ; \operatorname{TD} 8=\operatorname{zeros}(\mathrm{M}, 1) ; \mathrm{BLV} 8=\operatorname{zero}$ $\mathrm{S}(\mathrm{M}, 1)$;

$\mathrm{BL} 8=\operatorname{zeros}(\mathrm{M}, 1) ; \operatorname{BDV} 8=\operatorname{zeros}(\mathrm{M}, 1) ; \mathrm{BD} 8=\operatorname{zeros}(\mathrm{M}, 1) ; \mathrm{PV} 8=\operatorname{zeros}(\mathrm{M}, 1) ; \mathrm{P} 8=\operatorname{zeros}(\mathrm{M}$ , 1 ) ;

$\operatorname{TLV} 9=\operatorname{zeros}(\mathrm{M}, 1) ; \mathrm{TL} 9=\operatorname{zeros}(\mathrm{M}, 1) ; \operatorname{TDV} 9=\operatorname{zeros}(\mathrm{M}, 1) ; \operatorname{TD} 9=\operatorname{zeros}(\mathrm{M}, 1) ; \mathrm{BLV} 9=\operatorname{zero}$ $\mathrm{S}(\mathrm{M}, 1)$; 
$\mathrm{BL} 9=\operatorname{zeros}(\mathrm{M}, 1) ; \mathrm{BDV} 9=\operatorname{zeros}(\mathrm{M}, 1) ; \mathrm{BD} 9=\operatorname{zeros}(\mathrm{M}, 1) ; \mathrm{PV} 9=\operatorname{zeros}(\mathrm{M}, 1) ; \mathrm{P} 9=\operatorname{zeros}(\mathrm{M}$ , 1) ;

$\operatorname{TLV} 10=\operatorname{zeros}(\mathrm{M}, 1) ; \operatorname{TL} 10=\operatorname{zeros}(\mathrm{M}, 1) ; \operatorname{TDV} 10=\operatorname{zeros}(\mathrm{M}, 1) ; \operatorname{TD} 10=\operatorname{zeros}(\mathrm{M}, 1) ; \mathrm{BLV} 10$ $=\operatorname{zeros}(\mathrm{M}, 1)$;

$\operatorname{BL} 10=\operatorname{zeros}(\mathrm{M}, 1) ; \operatorname{BDV} 10=\operatorname{zeros}(\mathrm{M}, 1) ; \operatorname{BD} 10=\operatorname{zeros}(\mathrm{M}, 1) ; \operatorname{PV} 10=\operatorname{zeros}(\mathrm{M}, 1) ; \mathrm{P} 10=\mathrm{ze}$ $\operatorname{ros}(\mathrm{M}, 1)$;

$\operatorname{TLV} 11=\operatorname{zeros}(\mathrm{M}, 1) ; \operatorname{TL} 11=\operatorname{zeros}(\mathrm{M}, 1) ; \operatorname{TDV} 11=\operatorname{zeros}(\mathrm{M}, 1) ; \operatorname{TD} 11=\operatorname{zeros}(\mathrm{M}, 1) ; \mathrm{BLV} 11$ $=\operatorname{zeros}(\mathrm{M}, 1)$;

$\operatorname{BL} 11=\operatorname{zeros}(\mathrm{M}, 1) ; \operatorname{BDV} 11=\operatorname{zeros}(\mathrm{M}, 1) ; \operatorname{BD} 11=\operatorname{zeros}(\mathrm{M}, 1) ; \operatorname{PV} 11=\operatorname{zeros}(\mathrm{M}, 1) ; \mathrm{P} 11=\mathrm{ze}$ $\operatorname{ros}(\mathrm{M}, 1) ;$

$\operatorname{TLV} 12=\operatorname{zeros}(\mathrm{M}, 1) ; \operatorname{TL1}=\operatorname{zeros}(\mathrm{M}, 1) ; \operatorname{TDV} 12=\operatorname{zeros}(\mathrm{M}, 1) ; \operatorname{TD} 12=\operatorname{zeros}(\mathrm{M}, 1) ; \operatorname{BLV} 12$ $=\operatorname{zeros}(\mathrm{M}, 1)$;

$\operatorname{BL1} 2=\operatorname{zeros}(\mathrm{M}, 1) ; \operatorname{BDV} 12=\operatorname{zeros}(\mathrm{M}, 1) ; \operatorname{BD} 12=\operatorname{zeros}(\mathrm{M}, 1) ; \operatorname{PV} 12=\operatorname{zeros}(\mathrm{M}, 1) ; \mathrm{P} 12=\mathrm{ze}$ $\operatorname{ros}(\mathrm{M}, 1)$;

$\operatorname{TLV13}=\operatorname{zeros}(\mathrm{M}, 1) ; \operatorname{TL13}=\operatorname{zeros}(\mathrm{M}, 1) ; \operatorname{TDV} 13=\operatorname{zeros}(\mathrm{M}, 1) ; \operatorname{TD} 13=\operatorname{zeros}(\mathrm{M}, 1) ; \mathrm{BLV} 13$ $=\operatorname{zeros}(\mathrm{M}, 1)$;

$\operatorname{BL13}=\operatorname{zeros}(\mathrm{M}, 1) ; \operatorname{BDV} 13=\operatorname{zeros}(\mathrm{M}, 1) ; \operatorname{BD13}=\operatorname{zeros}(\mathrm{M}, 1) ; \operatorname{PV13}=\operatorname{zeros}(\mathrm{M}, 1) ; \mathrm{P} 13=\mathrm{ze}$ $\operatorname{ros}(\mathrm{M}, 1) ;$

$\operatorname{TLV} 14=\operatorname{zeros}(\mathrm{M}, 1) ; \operatorname{TL} 14=\operatorname{zeros}(\mathrm{M}, 1) ; \operatorname{TDV} 14=\operatorname{zeros}(\mathrm{M}, 1) ; \operatorname{TD} 14=\operatorname{zeros}(\mathrm{M}, 1) ; \mathrm{BLV} 14$ $=\operatorname{zeros}(\mathrm{M}, 1)$;

$\operatorname{BL14}=\operatorname{zeros}(\mathrm{M}, 1) ; \operatorname{BDV} 14=\operatorname{zeros}(\mathrm{M}, 1) ; \operatorname{BD1} 4=\operatorname{zeros}(\mathrm{M}, 1) ; \operatorname{PV1} 4=\operatorname{zeros}(\mathrm{M}, 1) ; \mathrm{P} 14=\mathrm{ze}$ $\operatorname{ros}(\mathrm{M}, 1)$;

$\operatorname{TLV} 15=\operatorname{zeros}(\mathrm{M}, 1) ; \operatorname{TL} 15=\operatorname{zeros}(\mathrm{M}, 1) ; \operatorname{TDV} 15=\operatorname{zeros}(\mathrm{M}, 1) ; \operatorname{TD} 15=\operatorname{zerOs}(\mathrm{M}, 1) ; \mathrm{BLV} 15$ $=\operatorname{zeros}(\mathrm{M}, 1)$;

$\operatorname{BL15}=\operatorname{zeros}(\mathrm{M}, 1) ; \operatorname{BDV} 15=\operatorname{zeros}(\mathrm{M}, 1) ; \operatorname{BD} 15=\operatorname{zeros}(\mathrm{M}, 1) ; \operatorname{PV} 15=\operatorname{zeros}(\mathrm{M}, 1) ; \mathrm{P} 15=\mathrm{ze}$ $\operatorname{ros}(\mathrm{M}, 1) ;$

$\operatorname{TLV} 16=\operatorname{zeros}(\mathrm{M}, 1) ; \operatorname{TL1} 16=\operatorname{zeros}(\mathrm{M}, 1) ; \operatorname{TDV} 16=\operatorname{zeros}(\mathrm{M}, 1) ; \operatorname{TD} 16=\operatorname{zeros}(\mathrm{M}, 1) ; \mathrm{BLV} 16$ $=\operatorname{zeros}(\mathrm{M}, 1)$;

$\operatorname{BL1} 16=\operatorname{zeros}(\mathrm{M}, 1) ; \operatorname{BDV} 16=\operatorname{zeros}(\mathrm{M}, 1) ; \operatorname{BD} 16=\operatorname{zeros}(\mathrm{M}, 1) ; \operatorname{PV} 16=\operatorname{zeros}(\mathrm{M}, 1) ; \mathrm{P} 16=\mathrm{ze}$ $\operatorname{ros}(\mathrm{M}, 1)$;

$\operatorname{TLV} 17=\operatorname{zeros}(\mathrm{M}, 1) ; \operatorname{TL} 17=\operatorname{zeros}(\mathrm{M}, 1) ; \operatorname{TDV} 17=\operatorname{zeros}(\mathrm{M}, 1) ; \operatorname{TD} 17=\operatorname{zeros}(\mathrm{M}, 1) ; \mathrm{BLV} 17$ = zeros $(\mathrm{M}, 1)$;

$\operatorname{BL1} 7=\operatorname{zeros}(\mathrm{M}, 1) ; \operatorname{BDV} 17=\operatorname{zeros}(\mathrm{M}, 1) ; \operatorname{BD} 17=\operatorname{zeros}(\mathrm{M}, 1) ; \operatorname{PV} 17=\operatorname{zeros}(\mathrm{M}, 1) ; \mathrm{P} 17=\mathrm{ze}$ $\operatorname{ros}(\mathrm{M}, 1)$;

$\operatorname{TLV} 18=\operatorname{zeros}(\mathrm{M}, 1) ; \operatorname{TL} 18=\operatorname{zeros}(\mathrm{M}, 1) ; \operatorname{TDV} 18=\operatorname{zeros}(\mathrm{M}, 1) ; \operatorname{TD} 18=\operatorname{zeros}(\mathrm{M}, 1) ; \mathrm{BLV} 18$ $=\operatorname{zeros}(\mathrm{M}, 1)$;

$\operatorname{BL18}=\operatorname{zeros}(\mathrm{M}, 1) ; \operatorname{BDV} 18=\operatorname{zeros}(\mathrm{M}, 1) ; \operatorname{BD} 18=\operatorname{zeros}(\mathrm{M}, 1) ; \operatorname{PV} 18=\operatorname{zeros}(\mathrm{M}, 1) ; \mathrm{P} 18=\mathrm{ze}$ $\operatorname{ros}(\mathrm{M}, 1)$;

$\operatorname{TLV} 19=\operatorname{zeros}(\mathrm{M}, 1) ; \operatorname{TL} 19=\operatorname{zeros}(\mathrm{M}, 1) ; \operatorname{TDV} 19=\operatorname{zeros}(\mathrm{M}, 1) ; \operatorname{TD} 19=\operatorname{zerOs}(\mathrm{M}, 1) ; \mathrm{BLV} 19$ $=\operatorname{zeros}(\mathrm{M}, 1)$;

$\operatorname{BL1} 19=\operatorname{zeros}(\mathrm{M}, 1) ; \operatorname{BDV} 19=\operatorname{zeros}(\mathrm{M}, 1) ; \operatorname{BD} 19=\operatorname{zeros}(\mathrm{M}, 1) ; \operatorname{PV1}=\operatorname{zeros}(\mathrm{M}, 1) ; \mathrm{P} 19=\mathrm{ze}$ $\operatorname{ros}(\mathrm{M}, 1)$;

$\operatorname{TLV} 20=\operatorname{zeros}(\mathrm{M}, 1) ; \operatorname{TL} 20=\operatorname{zeros}(\mathrm{M}, 1) ; \operatorname{TDV} 20=\operatorname{zeros}(\mathrm{M}, 1) ; \operatorname{TD} 20=\operatorname{zeros}(\mathrm{M}, 1) ; \mathrm{BLV} 20$ =zeros $(\mathrm{M}, 1)$;

$\mathrm{BL} 20=\operatorname{zeros}(\mathrm{M}, 1) ; \operatorname{BDV} 20=\operatorname{zeros}(\mathrm{M}, 1) ; \mathrm{BD} 20=\operatorname{zeros}(\mathrm{M}, 1) ; \mathrm{PV} 20=\operatorname{zeros}(\mathrm{M}, 1) ; \mathrm{P} 20=\mathrm{ze}$ $\operatorname{ros}(\mathrm{M}, 1)$;

$\operatorname{TLV} 21=\operatorname{zeros}(\mathrm{M}, 1) ; \operatorname{TL} 21=\operatorname{zeros}(\mathrm{M}, 1) ; \operatorname{TDV} 21=\operatorname{zeros}(\mathrm{M}, 1) ; \operatorname{TD} 21=\operatorname{zeros}(\mathrm{M}, 1) ; \mathrm{BLV} 21$ $=\operatorname{zeros}(\mathrm{M}, 1)$;

$\operatorname{BL} 21=\operatorname{zeros}(\mathrm{M}, 1) ; \operatorname{BDV} 21=\operatorname{zeros}(\mathrm{M}, 1) ; \mathrm{BD} 21=\operatorname{zeros}(\mathrm{M}, 1) ; \operatorname{PV} 21=\operatorname{zeros}(\mathrm{M}, 1) ; \mathrm{P} 21=\mathrm{ze}$ $\operatorname{ros}(\mathrm{M}, 1)$;

$\operatorname{TLV} 22=\operatorname{zeros}(\mathrm{M}, 1) ; \operatorname{TL} 22=\operatorname{zeros}(\mathrm{M}, 1) ; \operatorname{TDV} 22=\operatorname{zeros}(\mathrm{M}, 1) ; \operatorname{TD} 22=\operatorname{zeros}(\mathrm{M}, 1) ; \mathrm{BLV} 22$ $=\operatorname{zeros}(\mathrm{M}, 1)$;

$\operatorname{BL} 22=\operatorname{zeros}(\mathrm{M}, 1) ; \operatorname{BDV} 22=\operatorname{zeros}(\mathrm{M}, 1) ; \operatorname{BD} 22=\operatorname{zeros}(\mathrm{M}, 1) ; \mathrm{PV} 22=\operatorname{zeros}(\mathrm{M}, 1) ; \mathrm{P} 22=\mathrm{ze}$ $\operatorname{ros}(\mathrm{M}, 1)$;

$\operatorname{TLV} 23=\operatorname{zeros}(\mathrm{M}, 1) ; \operatorname{TL} 23=\operatorname{zeros}(\mathrm{M}, 1) ; \operatorname{TDV} 23=\operatorname{zeros}(\mathrm{M}, 1) ; \operatorname{TD} 23=\operatorname{zeros}(\mathrm{M}, 1) ; \mathrm{BLV} 23$ $=\operatorname{zeros}(\mathrm{M}, 1)$; 
$\mathrm{BL} 23=\operatorname{zeros}(\mathrm{M}, 1) ; \operatorname{BDV} 23=\operatorname{zeros}(\mathrm{M}, 1) ; \mathrm{BD} 23=\operatorname{zeros}(\mathrm{M}, 1) ; \mathrm{PV} 23=\operatorname{zeros}(\mathrm{M}, 1) ; \mathrm{P} 23=\mathrm{ze}$ $\operatorname{ros}(\mathrm{M}, 1)$;

$\operatorname{TLV} 24=\operatorname{zeros}(\mathrm{M}, 1) ; \mathrm{TL} 24=\operatorname{zeros}(\mathrm{M}, 1) ; \operatorname{TDV} 24=\operatorname{zeros}(\mathrm{M}, 1) ; \operatorname{TD} 24=\operatorname{zeros}(\mathrm{M}, 1) ; \mathrm{BLV} 24$ $=\operatorname{zeros}(\mathrm{M}, 1)$;

BL2 4=zeros $(\mathrm{M}, 1) ; \operatorname{BDV} 24=\operatorname{zeros}(\mathrm{M}, 1) ; \operatorname{BD} 24=\operatorname{zeros}(\mathrm{M}, 1) ; \operatorname{PV} 24=\operatorname{zeros}(\mathrm{M}, 1) ; \mathrm{P} 24=\mathrm{ze}$ $\operatorname{ros}(\mathrm{M}, 1)$;

$\operatorname{TLV} 25=\operatorname{zeros}(\mathrm{M}, 1) ; \mathrm{TL} 25=\operatorname{zeros}(\mathrm{M}, 1) ; \operatorname{TDV} 25=\operatorname{zeros}(\mathrm{M}, 1) ; \operatorname{TD} 25=\operatorname{zeros}(\mathrm{M}, 1) ; \mathrm{BLV} 25$

$=\operatorname{zeros}(\mathrm{M}, 1)$;

$\mathrm{BL} 25=\operatorname{zeros}(\mathrm{M}, 1) ; \operatorname{BDV} 25=\operatorname{zeros}(\mathrm{M}, 1) ; \operatorname{BD} 25=\operatorname{zeros}(\mathrm{M}, 1) ; \operatorname{PV} 25=\operatorname{zeros}(\mathrm{M}, 1) ; \mathrm{P} 25=\mathrm{ze}$ $\operatorname{ros}(\mathrm{M}, 1)$;

$\operatorname{TLV} 26=\operatorname{zeros}(\mathrm{M}, 1) ; \operatorname{TL} 26=\operatorname{zeros}(\mathrm{M}, 1) ; \operatorname{TDV} 26=\operatorname{zeros}(\mathrm{M}, 1) ; \operatorname{TD} 26=\operatorname{zeros}(\mathrm{M}, 1) ; \mathrm{BLV} 26$ $=\operatorname{zeros}(\mathrm{M}, 1)$;

$\operatorname{BL} 26=\operatorname{zeros}(\mathrm{M}, 1) ; \operatorname{BDV} 26=\operatorname{zeros}(\mathrm{M}, 1) ; \operatorname{BD} 26=\operatorname{zeros}(\mathrm{M}, 1) ; \mathrm{PV} 26=\operatorname{zeros}(\mathrm{M}, 1) ; \mathrm{P} 26=\mathrm{ze}$ $\operatorname{ros}(\mathrm{M}, 1)$;

$\operatorname{TLV} 27=\operatorname{zeros}(\mathrm{M}, 1) ; \operatorname{TL} 27=\operatorname{zeros}(\mathrm{M}, 1) ; \operatorname{TDV} 27=\operatorname{zeros}(\mathrm{M}, 1) ; \operatorname{TD} 27=\operatorname{zeros}(\mathrm{M}, 1) ; \mathrm{BLV} 27$ $=\operatorname{zeros}(\mathrm{M}, 1)$;

$\operatorname{BL} 27=\operatorname{zeros}(M, 1) ; \operatorname{BDV} 27=\operatorname{zeros}(M, 1) ; \operatorname{BD} 27=\operatorname{zeros}(M, 1) ; \operatorname{PV} 27=\operatorname{zeros}(M, 1) ; P 27=\mathrm{ze}$ $\operatorname{ros}(\mathrm{M}, 1)$;

$\operatorname{TLV} 28=\operatorname{zeros}(\mathrm{M}, 1) ; \operatorname{TL} 28=\operatorname{zeros}(\mathrm{M}, 1) ; \operatorname{TDV} 28=\operatorname{zeros}(\mathrm{M}, 1) ; \operatorname{TD} 28=\operatorname{zeros}(\mathrm{M}, 1) ; \mathrm{BLV} 28$ $=\operatorname{zeros}(\mathrm{M}, 1)$;

$\mathrm{BL} 28=\operatorname{zeros}(\mathrm{M}, 1) ; \operatorname{BDV} 28=\operatorname{zeros}(\mathrm{M}, 1) ; \operatorname{BD} 28=\operatorname{zeros}(\mathrm{M}, 1) ; \mathrm{PV} 28=\operatorname{zeros}(\mathrm{M}, 1) ; \mathrm{P} 28=\mathrm{ze}$ $\operatorname{ros}(\mathrm{M}, 1)$;

TLV29=zeros $(M, 1) ; \operatorname{TL} 29=\operatorname{zeros}(M, 1) ; \operatorname{TDV} 29=\operatorname{zeros}(\mathrm{M}, 1) ; \operatorname{TD} 29=\operatorname{zeros}(\mathrm{M}, 1) ; B L V 29$

$=\operatorname{zeros}(\mathrm{M}, 1)$;

BL29=zeros $(M, 1) ; B D V 29=\operatorname{zeros}(M, 1) ; B D 29=\operatorname{zeros}(M, 1) ; P V 29=\operatorname{zeros}(\mathrm{M}, 1) ; \mathrm{P} 29=\mathrm{ze}$ $\operatorname{ros}(\mathrm{M}, 1)$;

$\operatorname{TLV} 30=\operatorname{zeros}(\mathrm{M}, 1) ; \operatorname{TL} 30=\operatorname{zeros}(\mathrm{M}, 1) ; \operatorname{TDV} 30=\operatorname{zeros}(\mathrm{M}, 1) ; \operatorname{TD} 30=\operatorname{zeros}(\mathrm{M}, 1) ; \mathrm{BLV} 30$ $=\operatorname{zeros}(\mathrm{M}, 1)$;

$\mathrm{BL} 30=\operatorname{zeros}(\mathrm{M}, 1) ; \operatorname{BDV} 30=\operatorname{zeros}(\mathrm{M}, 1) ; \mathrm{BD} 30=\operatorname{zeros}(\mathrm{M}, 1) ; \operatorname{PV} 30=\operatorname{zeros}(\mathrm{M}, 1) ; \mathrm{P} 30=\mathrm{ze}$ $\operatorname{ros}(\mathrm{M}, 1)$;

$\operatorname{TLV} 31=\operatorname{zeros}(\mathrm{M}, 1) ; \operatorname{TL} 31=\operatorname{zeros}(\mathrm{M}, 1) ; \operatorname{TDV} 31=\operatorname{zeros}(\mathrm{M}, 1) ; \operatorname{TD} 31=\operatorname{zeros}(\mathrm{M}, 1) ; \mathrm{BLV} 31$ $=\operatorname{zeros}(\mathrm{M}, 1)$;

$\mathrm{BL} 31=\operatorname{zeros}(\mathrm{M}, 1) ; \mathrm{BDV} 31=\operatorname{zeros}(\mathrm{M}, 1) ; \mathrm{BD} 31=\operatorname{zeros}(\mathrm{M}, 1) ; \operatorname{PV} 31=\operatorname{zeros}(\mathrm{M}, 1) ; \mathrm{P} 31=\mathrm{ze}$ $\operatorname{ros}(\mathrm{M}, 1) ; \mathrm{x}=\operatorname{zeros}(\mathrm{M}, 1)$;

osplit data matrix into arrays for TL, TD, BL, BD, and P0-31

for count $=1: \mathrm{M}$

TLV0 $($ count, 1$)=$ data 0. data $($ count, 2$) ;$ TL0 $($ count, 1$)=$ data 0. data $($ count, 3$) ;$ BLV0 $($ count, 1$)=$ data 0. data $($ count, 4$)$;

BLO $($ count, 1$)=$ data 0. data $($ count, 5$) ;$ TDV0 $($ count, 1$)=$ data 0. data $($ count, 6$) ;$ TD0 ( count, 1$)=$ data 0. data $($ count, 7$)$;

BDV0 $($ count, 1$)=$ data 0. data $($ count, 8$) ; B D 0($ count, 1$)=\operatorname{data0} \cdot$ data $(\operatorname{count}, 9) ;$ PV0 ( count, 1$)=\operatorname{data} 0$. data $($ count, 8$)$;

P0 (count, 1$)=$ data 0. data $($ count, 9$) ; T L V 1($ count, 1$)=\operatorname{data1}$.data $($ count, 2$) ;$ TL1 (c ount, 1$)=$ data 1 . data $($ count, 3$)$;

BLV1 ( count, 1$)=$ data1.data $($ count, 4$) ; B L 1($ count, 1$)=$ data1.data $(\operatorname{count}, 5)$; TD1 ( count, 1$)=$ data 1 . data $($ count, 6$)$;

TD1 ( count, 1) =data1.data ( count, 7$)$; BDV1 ( count, 1) =data1.data $(\operatorname{count}, 8)$; BD1 ( count, 1$)=$ datal. data $($ count, 9$)$;

PV1 ( count, 1)=data1.data ( count, 8);P1 (count, 1)=data1.data ( count, 9) ; TLV2 (c ount, 1$)=\operatorname{data} 2$.data $($ count, 2$)$;

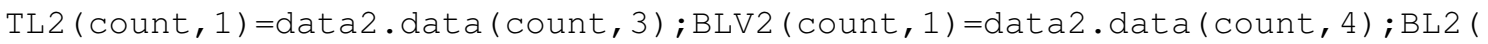
count, 1$)=$ data 2 . data $($ count, 5$)$;

TD2 $($ count, 1$)=\operatorname{data} 2$. data $($ count, 6$) ;$ TD2 $($ count, 1$)=\operatorname{data} 2$.data $($ count, 7$)$; BDV2 ( count, 1$)=$ data 2 .data $($ count, 8$)$;

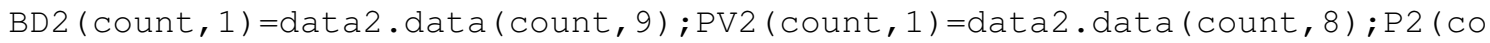
unt, 1) $=$ data 2 .data $($ count, 9$)$; 


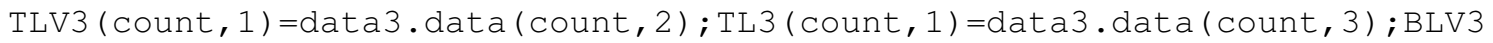
$($ count, 1$)=$ data3. data $($ count, 4$)$;

BL3 $($ count, 1$)=$ data 3. data $($ count, 5$) ; \operatorname{TD} 3($ count, 1$)=$ data3. data $(\operatorname{count}, 6) ; \operatorname{TD} 3(\mathrm{c}$ ount, 1$)=$ data3. data $($ count, 7$)$;

BDV3 $($ count, 1$)=\operatorname{data3}$. data $($ count, 8$) ; B D 3($ count, 1$)=\operatorname{data3}$.data $($ count, 9$) ;$ PV3 ( count, 1$)=$ data 3 . data $($ count, 8$)$;

P3 (count, 1$)=$ data $3 . \operatorname{data}($ count, 9$) ; \operatorname{TLV} 4($ count, 1$)=\operatorname{data} 4 . \operatorname{data}(\operatorname{count}, 2) ; \mathrm{TL} 4$ ( c ount, 1$)=$ data 4 .data $($ count, 3$)$;

BLV4 $($ count, 1$)=\operatorname{data} 4 \cdot \operatorname{data}($ count, 4$) ; B L 4($ count, 1$)=\operatorname{data} 4 \cdot \operatorname{data}(\operatorname{count}, 5) ;$ TD 4( count, 1$)=$ data 4 . data $($ count, 6$)$;

TD4 $($ count, 1$)=$ data 4 .data $($ count, 7$) ; B D V 4($ count, 1$)=\operatorname{data} 4$. data $(\operatorname{count}, 8) ; B D 4($ count, 1$)=$ data 4 .data $($ count, 9$)$;

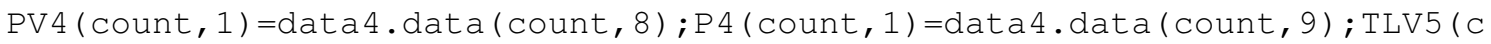
ount, 1$)=$ data 5 . data $($ count, 2$)$;

TL5 $($ count, 1$)=$ data 5. data $($ count, 3$) ;$ BLV5 $($ count, 1$)=\operatorname{data} 5$. data $(\operatorname{count}, 4) ; B L 5($ count, 1$)=$ data 5 . data $($ count, 5$)$;

TD5 $($ count, 1$)=$ data 5. data $($ count, 6$) ; \operatorname{TD} 5($ count, 1$)=\operatorname{data} 5$. data $(\operatorname{count}, 7) ;$ BDV5 ( count, 1$)=$ data 5 . data $($ count, 8$)$;

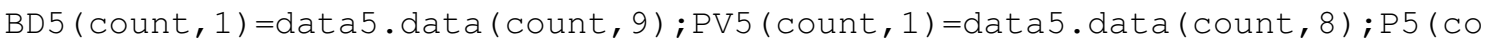
unt, 1$)=$ data 5 .data $($ count, 9$)$;

TLV6 $($ count, 1$)=$ data $6 \cdot$ data $($ count, 2$) ;$ TL6 $($ count, 1$)=$ data 6. data $($ count, 3$)$; BLV6 $($ count, 1$)=$ data 6 . data $($ count, 4$)$;

BL6 $($ count, 1$)=$ data 6. data $($ count, 5$) ; \operatorname{TD} 6($ count, 1$)=$ data 6. data $(\operatorname{count}, 6) ; \operatorname{TD} 6$ (c ount, 1$)=$ data 6 . data $($ count, 7$)$;

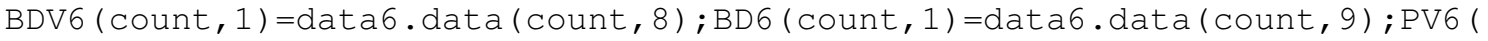
count, 1$)=$ data 6 . data $($ count, 8$)$;

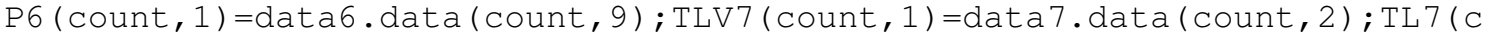
ount, 1$)=$ data 7 . data $($ count, 3$)$;

BLV7 (count, 1$)=$ data 7 .data $($ count, 4$) ; B L 7($ count, 1$)=$ data 7. data $($ count, 5$) ;$ TD 7 ( count, 1$)=$ data 7 . data $($ count, 6$)$;

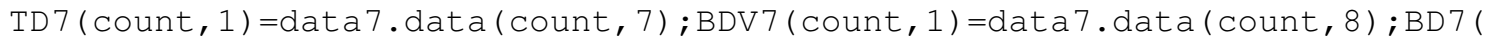
count, 1$)=\operatorname{data} 7$. data $($ count, 9$)$;

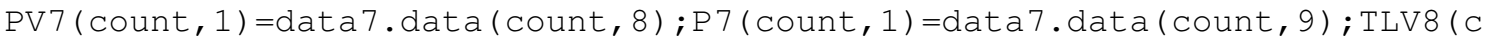
ount, 1$)=$ data 8 .data $($ count, 2$)$;

TL8 $($ count, 1$)=$ data 8 .data $($ count , 3$) ; B L V 8($ count, 1$)=$ data 8. data $(\operatorname{count}, 4) ; B L 8($ count, 1$)=$ data 8 .data $($ count, 5$)$;

TD8 $($ count, 1$)=$ data $8 \cdot \operatorname{data}(\operatorname{count}, 6) ;$ TD8 $($ count, 1$)=\operatorname{data} 8 \cdot \operatorname{data}(\operatorname{count}, 7) ;$ BDV8 ( count, 1$)=$ data 8 . data $($ count, 8$)$;

BD8 $($ count, 1$)=$ data 8 .data $($ count, 9$) ; P V 8($ count, 1$)=\operatorname{data} 8 . \operatorname{data}(\operatorname{count}, 8) ; \mathrm{P} 8(\mathrm{co}$ unt, 1$)=\operatorname{data} 8$.data $($ count, 9$)$;

TLV9 $($ count, 1$)=$ data 9. data $($ count, 2$) ;$ TL9 $($ count, 1$)=$ data 9. data $($ count, 3$)$; BLV9 ( count, 1$)=$ data9. data $($ count, 4$)$;

BL9 $($ count, 1$)=$ data 9. data $($ count, 5$) ;$ TD9 $($ count, 1$)$ =data9. data $($ count, 6$) ;$ TD9 (c ount, 1$)=$ data 9 . data $($ count, 7$)$;

BDV9 $($ count, 1$)=$ data9. data $($ count, 8$) ; B D 9($ count, 1$)=$ data9. data $(\operatorname{count}, 9) ;$ PV9 ( count, 1$)=$ data 9 . data $($ count, 8$)$;

P9 $($ count, 1$)=$ data 9. data $($ count, 9$) ;$ TLV10 $($ count, 1$)=$ data10.data $($ count, 2$)$; TL 1 $0($ count, 1$)=$ data10. data $($ count, 3$)$;

BLV10 ( count, 1$)=$ data10.data $($ count, 4$)$; BL10 (count, 1$)=\operatorname{data10}$.data $(\operatorname{count}, 5)$; TD10 ( count, 1$)=\operatorname{data10}$. data $($ count, 6$)$;

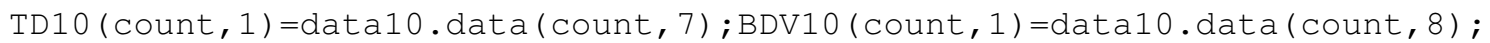
BD10 ( count, 1$)=$ data10.data $($ count, 9$)$;

PV10 ( count, 1$)=$ data10.data $($ count, 8$) ; P 10($ count, 1$)=$ data10.data $($ count, 9$)$; TL V11 ( count, 1$)=$ data11. data $($ count, 2$)$;

TL11 (count, 1$)=$ data11.data $($ count, 3$)$; BLV11 ( count, 1$)=\operatorname{data11}$.data $(\operatorname{count}, 4)$; BL11 $($ count, 1$)=\operatorname{data11}$.data $($ count , 5) ; 
TD11 ( count, 1$)=$ data11.data $($ count, 6$) ; \operatorname{TD1} 1($ count, 1$)=\operatorname{data11} \cdot \operatorname{data}($ count, 7$) ; B$ DV11 ( count, 1$)=$ data11. data $($ count, 8$)$;

BD11 ( count, 1$)=$ data11.data $($ count, 9$) ; P V 11($ count, 1$)=\operatorname{data11}$.data $(\operatorname{count}, 8) ; P$ 11 ( count, 1$)=$ data11. data ( count, 9$)$;

TLV12 (count, 1$)$ data12. data $($ count, 2$) ;$ TL12 (count, 1$)=\operatorname{data12} \cdot \operatorname{data}(\operatorname{count}, 3)$; BLV12 ( count, 1$)=$ data12. data $($ count, 4$)$;

BL12 $($ count, 1$)=\operatorname{data12}$.data $($ count, 5$) ; \operatorname{TD} 12($ count, 1$)=\operatorname{data12} \cdot \operatorname{data}(\operatorname{count}, 6) ; \mathrm{T}$ D12 ( count, 1$)=$ data12. data $($ count , 7$)$;

BDV12 (count, 1$)=\operatorname{data12}$.data $($ count, 8$) ; B D 12($ count, 1$)=\operatorname{data12} \cdot \operatorname{data}(\operatorname{count}, 9) ;$ PV12 (count, 1$)=$ data12. data $($ count, 8$)$;

P12 ( count, 1$)=$ data12. data $($ count, 9$) ; \operatorname{TLV13}($ count, 1$)=\operatorname{data13} \cdot \operatorname{data}(\operatorname{count}, 2) ; \mathrm{T}$ L13 ( count, 1$)=$ data13. data $($ count, 3$)$;

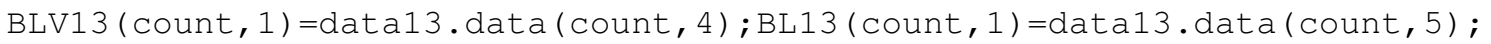
TD13 ( count, 1$)=$ data13. data $($ count, 6$) ;$

TD13 (count, 1) =data13. data $($ count, 7$) ; \operatorname{BDV13}($ count, 1$)=\operatorname{data13} \cdot \operatorname{data}(\operatorname{count}, 8)$; BD13 ( count, 1) =data13. data $($ count, 9$)$;

PV13 (count, 1$)=$ data13.data $($ count, 8$) ; P 13($ count, 1$)=$ data13.data $($ count, 9$)$; TL V14 ( count, 1$)=$ data14. data $($ count, 2$)$;

TL14 (count, 1$)=$ data14.data $($ count, 3$) ; B L V 14($ count, 1$)=\operatorname{data14} \cdot \operatorname{data}(\operatorname{count}, 4)$; BL14 (count, 1$)=\operatorname{data14}$. data $($ count, 5$)$;

TD14 (count, 1$)=$ data14.data $($ count, 6$) ; \operatorname{TD1} 4($ count, 1$)=\operatorname{data14} \cdot \operatorname{data}(\operatorname{count}, 7) ; B$ DV14 ( count, 1$)=\operatorname{data1} 4$. data $($ count, 8$)$;

BD14 (count, 1)=data14.data $($ count, 9$) ; P V 14($ count, 1$)=\operatorname{data14} \cdot \operatorname{data}(\operatorname{count}, 8) ; \mathrm{P}$ $14($ count, 1$)=$ data14. data $($ count, 9$)$;

TLV15 ( count, 1$)=\operatorname{data15}$.data $($ count, 2$) ;$ TL15 $($ count, 1$)=\operatorname{data15} \cdot \operatorname{data}(\operatorname{count}, 3)$; BLV15 ( count, 1$)=$ data15. data $($ count, 4$)$;

BL15 $($ count, 1$)=$ data15. data $($ count, 5$) ;$ TD15 $($ count, 1$)=\operatorname{data15} \cdot \operatorname{data}(\operatorname{count}, 6) ; \mathrm{T}$ D15 ( count, 1$)=$ data15. data $($ count, 7$)$;

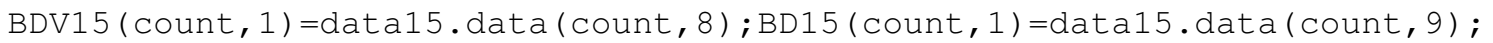
PV15 (count, 1) =data15.data (count, 8$)$;

P15 $($ count, 1$)=$ data15. data $($ count, 9$) ; \operatorname{TLV} 16($ count, 1$)=\operatorname{data16} \cdot \operatorname{data}(\operatorname{count}, 2) ; \mathrm{T}$ L16 ( count, 1$)=$ data16. data $($ count, 3$)$;

BLV16 ( count, 1) =data16.data $($ count, 4$) ; B L 16($ count, 1$)=\operatorname{data16} \cdot \operatorname{data}(\operatorname{count}, 5)$; TD16 ( count, 1$)=\operatorname{data16}$. data $($ count, 6$) ;$

TD16 $($ count, 1$)=$ data16.data $($ count, 7$) ; \operatorname{BDV} 16($ count, 1$)=\operatorname{data16} \cdot \operatorname{data}(\operatorname{count}, 8) ;$ BD16 $($ count, 1$)=\operatorname{data16}$. data $($ count, 9$)$;

PV16 $($ count, 1$)=$ data16.data $($ count, 8$) ; P 16($ count, 1$)=$ data16.data $($ count, 9$)$; TL V17 ( count, 1$)=$ data17. data $($ count, 2$)$;

TL1 $7($ count, 1$)=$ data1 7 .data $($ count, 3$) ; B L V 17($ count, 1$)=\operatorname{data17} \cdot \operatorname{data}($ count, 4$) ;$ BL17 (count, 1$)=\operatorname{data17}$. data $($ count, 5$)$;

TD17 $($ count, 1$)=$ data1 7 .data $($ count, 6$) ; \operatorname{TD1} 7($ count, 1$)=\operatorname{data1} 7$.data $($ count, 7$) ; B$ DV1 7 ( count, 1$)=\operatorname{data17}$. data $($ count, 8$)$;

BD17 $($ count, 1$)=$ data1 7 .data $($ count, 9);PV1 $7($ count, 1$)=\operatorname{data17} \cdot \operatorname{data}(\operatorname{count}, 8)$; P $17($ count, 1$)=$ data1 7 . data $($ count, 9$)$;

TLV18 $($ count, 1$)=\operatorname{data18} \cdot \operatorname{data}($ count, 2$) ;$ TL18 $($ count, 1$)=\operatorname{data18} \cdot \operatorname{data}(\operatorname{count}, 3)$; BLV18 ( count, 1$)=$ data18. data $($ count, 4$)$;

BL18 $($ count, 1$)=$ data18.data $($ count, 5$) ; \operatorname{TD} 18(\operatorname{count}, 1)=\operatorname{data18} \cdot \operatorname{data}(\operatorname{count}, 6) ; \mathrm{T}$ D18 $($ count, 1$)=\operatorname{data18}$. data $($ count, 7$)$;

BDV18 ( count, 1$)=$ data18. data $(\operatorname{count}, 8) ; B D 18(\operatorname{count}, 1)=\operatorname{data18} \cdot \operatorname{data}(\operatorname{count}, 9)$; PV18 (count, 1$)=$ data18. data $($ count, 8$)$;

P18 (count, 1$)=$ data18. data $($ count, 9$) ; \operatorname{TLV} 19(\operatorname{count}, 1)=\operatorname{data19} \cdot \operatorname{data}(\operatorname{count}, 2) ; \mathrm{T}$ L19 ( count, 1$)=$ data19. data $($ count, 3$)$;

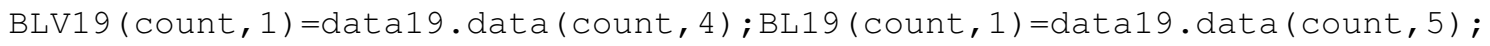
TD19 $($ count, 1$)=$ data19. data $($ count, 6$)$;

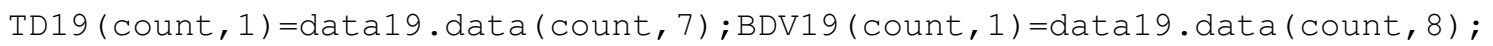
BD19 $($ count, 1$)=\operatorname{data19} \cdot \operatorname{data}($ count, 9$)$; 
PV19 (count, 1$)=$ data19.data $($ count, 8$) ; P 19($ count, 1$)=$ data19.data $($ count, 9$) ;$ TL V20 ( count, 1$)=$ data20.data $($ count, 2$)$;

TL20 ( count, 1$)=\operatorname{data} 20 . \operatorname{data}($ count, 3$) ; B L V 20($ count, 1$)=\operatorname{data} 20 . \operatorname{data}(\operatorname{count}, 4)$; BL20 ( count, 1$)=\operatorname{data20}$. data $($ count, 5$) ;$

TD20 $($ count, 1$)=\operatorname{data} 20 \cdot \operatorname{data}($ count, 6$) ; \operatorname{TD} 20($ count, 1$)=\operatorname{data} 20 \cdot \operatorname{data}(\operatorname{count}, 7) ; B$ DV20 ( count, 1$)=$ data20. data $($ count, 8$)$;

BD20 ( count, 1$)=$ data20.data $($ count, 9$) ; P V 20($ count, 1$)=\operatorname{data20} \cdot$ data $(\operatorname{count}, 8) ; P$ $20($ count, 1$)=$ data20.data $($ count, 9$)$;

TLV21 ( count, 1) =data21. data $($ count, 2$)$; TL21 (count, 1$)=\operatorname{data21} \cdot \operatorname{data}(\operatorname{count}, 3)$; BLV21 ( count, 1$)=\operatorname{data21}$. data ( count, 4$)$;

BL21 ( count, 1$)=$ data21.data $($ count, 5$) ;$ TD21 ( count, 1$)=\operatorname{data21} \cdot \operatorname{data}(\operatorname{count}, 6) ; \mathrm{T}$ D21 ( count, 1$)=$ data21. data $($ count, 7$)$;

BDV21 ( count, 1$)=\operatorname{data} 21$.data $($ count, 8$) ; B D 21($ count, 1$)=\operatorname{data21.data~}(\operatorname{count}, 9) ;$ PV21 ( count, 1) =data21.data $($ count, 8$)$;

P21 ( count, 1$)=$ data21. data $($ count, 9$) ; \operatorname{TLV} 22($ count, 1$)=\operatorname{data22} \cdot \operatorname{data}(\operatorname{count}, 2) ; \mathrm{T}$ L22 ( count, 1$)=$ data22. data ( count, 3$)$;

BLV22 ( count, 1$)=\operatorname{data22}$. data $($ count, 4);BL22 (count, 1$)=\operatorname{data22} \cdot \operatorname{data}(\operatorname{count}, 5)$; TD22 ( count, 1$)=\operatorname{data22}$. data $($ count, 6$)$;

TD22 (count, 1$)=$ data22. data $($ count, 7$) ; \operatorname{BDV} 22($ count, 1$)=\operatorname{data22}$.data $(\operatorname{count}, 8)$; BD22 (count, 1$)=\operatorname{data22}$. data $($ count, 9$)$;

PV22 (count, 1$)=$ data2 2 .data $($ count, 8$) ;$ P22 $($ count, 1$)=$ data22.data $($ count, 9$)$; TL V23 ( count, 1$)=$ data23. data $($ count, 2$)$;

TL23 $($ count, 1$)=\operatorname{data23}$. data $($ count, 3$) ; \operatorname{BLV} 23($ count, 1$)=\operatorname{data23.}$ data $($ count, 4$) ;$ BL23 ( count, 1$)=$ data23. data $($ count, 5$)$;

TD23 (count, 1$)=$ data23.data $($ count, 6$) ; \operatorname{TD} 23($ count, 1$)=\operatorname{data23} \cdot \operatorname{data}(\operatorname{count}, 7) ; \mathrm{B}$ DV23 ( count, 1) =data23. data $($ count, 8$) ;$

BD23 (count, 1$)=$ data23. data $($ count, 9$) ;$ PV23 $($ count, 1$)=\operatorname{data23} \cdot$ data $(\operatorname{count}, 8) ; P$ $23($ count, 1$)=$ data23. data $($ count, 9$)$;

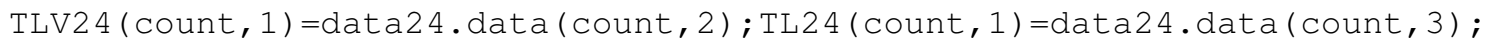
BLV24 ( count, 1$)=\operatorname{data24}$. data $($ count, 4$)$;

BL24 $($ count, 1$)=\operatorname{data} 24 \cdot \operatorname{data}($ count, 5$) ;$ TD24 $($ count, 1$)=\operatorname{data} 24 \cdot \operatorname{data}(\operatorname{count}, 6) ; \mathrm{T}$ D24 ( count, 1$)=$ data24. data $($ count, 7$)$;

BDV24 ( count, 1$)=\operatorname{data24} \cdot \operatorname{data}($ count, 8$) ; B D 24($ count, 1$)=\operatorname{data24} \cdot \operatorname{data}(\operatorname{count}, 9)$; PV24 (count, 1) =data24.data (count, 8$)$;

P24 (count, 1$)=\operatorname{data} 24 \cdot \operatorname{data}($ count, 9$) ; \mathrm{TLV} 25($ count, 1$)=\operatorname{data} 25 \cdot \operatorname{data}(\operatorname{count}, 2) ; \mathrm{T}$ L25 ( count, 1$)=$ data25. data ( count, 3$)$;

BLV25 (count, 1$)=\operatorname{data} 25$. data $($ count, 4$) ; B L 25($ count, 1$)=\operatorname{data25}$.data $(\operatorname{count}, 5)$; TD25 ( count, 1$)=\operatorname{data25}$. data $($ count, 6$)$;

TD25 (count, 1$)=$ data25.data $($ count, 7$) ; B D V 25($ count, 1$)=\operatorname{data25} \cdot \operatorname{data}(\operatorname{count}, 8) ;$ BD25 ( count, 1$)=\operatorname{data25}$. data $($ count, 9$) ;$

PV25 (count, 1$)=$ data 25 .data $($ count, 8$) ;$ P25 (count, 1$)=$ data25.data $($ count, 9$)$; TL V26 ( count, 1$)=$ data26. data ( count, 2$)$;

TL26 $($ count, 1$)=\operatorname{data} 26$.data $($ count, 3$) ; B L V 26($ count, 1$)=\operatorname{data26} \cdot \operatorname{data}(\operatorname{count}, 4)$; BL26 $($ count, 1$)=\operatorname{data26}$. data $($ count, 5$) ;$

TD26 $($ count, 1$)=$ data26.data $($ count, 6$) ;$ TD26 $($ count, 1$)=$ data26.data $($ count, 7$) ; B$ DV26 (count, 1$)=\operatorname{data26}$. data $($ count, 8$) ;$

BD26 $($ count, 1$)=\operatorname{data} 26 \cdot$ data $($ count, 9$) ; P V 26($ count, 1$)=\operatorname{data26} \cdot$ data $($ count, 8$) ; P$ $26($ count, 1$)=$ data26. data $($ count, 9$)$;

TLV27 ( count, 1) =data27.data $($ count, 2$)$; TL27 (count, 1$)=\operatorname{data27}$.data $(\operatorname{count}, 3)$; BLV27 ( count, 1$)=$ data27. data $($ count, 4$)$;

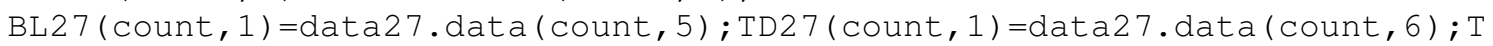
D27 ( count, 1$)=\operatorname{data27}$. data $($ count, 7$)$;

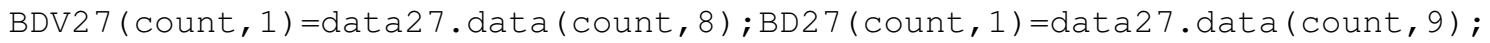
PV27 ( count, 1$)=\operatorname{data27}$. data $($ count, 8$)$;

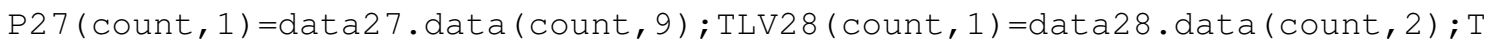
L28 ( count, 1$)=$ data28. data $($ count, 3$)$; 
BLV28 $($ count, 1$)=\operatorname{data} 28 \cdot \operatorname{data}($ count, 4$) ; B L 28($ count, 1$)=\operatorname{data28} \cdot \operatorname{data}(\operatorname{count}, 5)$; TD28 $($ count, 1$)=$ data28. data $($ count, 6$) ;$

TD28 $($ count, 1$)=\operatorname{data28} \cdot \operatorname{data}($ count, 7$) ; B D V 28($ count, 1$)=\operatorname{data28} \cdot \operatorname{data}(\operatorname{count}, 8) ;$ BD28 ( count, 1$)=\operatorname{data28}$. data $($ count, 9$) ;$

PV28 (count, 1$)=$ data28. data $($ count, 8$) ; P 28($ count, 1$)=$ data28.data $($ count, 9$) ;$ TL V29 ( count, 1$)=$ data29. data $($ count, 2$)$;

TL29 (count, 1$)=\operatorname{data29}$.data $($ count, 3$) ; B L V 29($ count, 1$)=\operatorname{data29} \cdot \operatorname{data}($ count, 4$)$; BL29 (count, 1$)=$ data29. data $($ count, 5$)$;

TD29 $($ count, 1$)=\operatorname{data29} \cdot \operatorname{data}($ count, 6$) ; \operatorname{TD} 29($ count, 1$)=\operatorname{data29} \cdot \operatorname{data}(\operatorname{count}, 7) ; B$ DV29 $($ count, 1$)=\operatorname{data29}$. data $($ count, 8$) ;$

BD29 $($ count, 1$)=\operatorname{data29}$.data $($ count, 9$) ;$ PV29 $($ count, 1$)=\operatorname{data29} \cdot$ data $(\operatorname{count}, 8) ; P$ $29($ count, 1$)=$ data 29 . data $($ count, 9$)$;

TLV30 ( count, 1$)=\operatorname{data} 30 . \operatorname{data}($ count, 2$)$; TL30 $($ count, 1$)=\operatorname{data30} \cdot \operatorname{data}(\operatorname{count}, 3)$; BLV30 ( count, 1$)=\operatorname{data30}$.data $($ count, 4$)$;

BL30 $($ count, 1$)=\operatorname{data} 30 . \operatorname{data}($ count, 5$) ; \operatorname{TD} 30($ count, 1$)=\operatorname{data} 30 \cdot \operatorname{data}(\operatorname{count}, 6) ; \mathrm{T}$ D30 ( count, 1$)=$ data30. data $($ count, 7$)$;

BDV30 ( count, 1$)=\operatorname{data} 30 . \operatorname{data}($ count, 8$) ; B D 30($ count, 1$)=\operatorname{data30} \cdot \operatorname{data}(\operatorname{count}, 9)$; PV30 ( count, 1$)=\operatorname{data30}$.data $($ count, 8$)$;

P30 ( count, 1$)=\operatorname{data} 30 \cdot \operatorname{data}($ count, 9$) ; \mathrm{TLV} 31($ count, 1$)=\operatorname{data3} 1 . \operatorname{data}(\operatorname{count}, 2) ; \mathrm{T}$ L31 ( count, 1$)=$ data31. data $($ count, 3$)$;

BLV31 ( count, 1)=data31.data $($ count, 4);BL31 (count, 1$)=\operatorname{data31} \cdot \operatorname{data}(\operatorname{count}, 5)$; TD31 ( count, 1$)=\operatorname{data3} 1$. data $($ count, 6$)$;

TD31 (count, 1$)=$ data31.data $($ count, 7$) ; \operatorname{BDV} 31($ count, 1$)=\operatorname{data3} 1 . \operatorname{data}(\operatorname{count}, 8) ;$ BD31 ( count, 1$)=\operatorname{data31}$. data $($ count, 9$)$;

PV31 $($ count, 1$)=\operatorname{data31}$.data $($ count, 8$) ; P 31($ count, 1$)=\operatorname{data3} 1 . \operatorname{data}(\operatorname{count}, 9) ; x($ count, 1 ) =count;

end

ocalculate statistics of Scientific Pressure data

$\mathrm{av} \_\mathrm{P} 0=$ mean $(\mathrm{P} 0) ; \mathrm{av} \_\mathrm{P} 1=\operatorname{mean}(\mathrm{P} 1) ; \mathrm{av} \_\mathrm{P} 2=\operatorname{mean}(\mathrm{P} 2) ; \mathrm{av} \_\mathrm{P} 3=$ mean $(\mathrm{P} 3) ; \mathrm{av} \_\mathrm{P} 4=\mathrm{mean}($ $\mathrm{P} 4) ; \mathrm{av} \_\mathrm{P} 5=\operatorname{mean}(\mathrm{P} 5) ; \mathrm{av} \_\mathrm{P} 6=\operatorname{mean}(\mathrm{P} 6)$;

av_P7=mean (P7); av_P8=mean (P8); av_P9=mean (P9); av_P10=mean (P10); av_P11=me an (P11); av_P12=mean (P12); av_P13=mean (P13);

av_P14=mean (P14); av_P15=mean (P15);av_P16=mean (P16);av_P17=mean (P17);av_ $\mathrm{P} 18=\operatorname{mean}(\mathrm{P} 18) ; \mathrm{av} \_\mathrm{P} 19=\operatorname{mean}(\mathrm{P} 19) ; \mathrm{av} \_\mathrm{P} 20=$ mean $(\mathrm{P} 20) ;$

av_P21=mean (P21); av_P22=mean (P22) ; av_P23=mean (P23); av_P24=mean (P24);av_ P25 =mean (P25); av_P26=mean (P26); av_P27=mean (P27); av_P28=mean (P28); av_P29=mean (P29); av_P30=mean (P30); av_P31=mean (P31);

standarddev_P $0=$ std $(\mathrm{P} 0)$; standarddev_P1=std ( 1 1) ; standarddev_P2=std (P2) ; st andarddev_P3 =std (P3); standarddev_P4 $=$ std (P4); standarddev_P5=std (P5) ; stan darddev_P $\overline{6}=$ std $(\mathrm{P} 6)$;

standarddev_P7=std (P7); standarddev_P8=std (P8); standarddev_P9=std (P9) ; st andarddev_P10 =std (P10); standarddev_P11=std (P11); standarddev_P12=std (P12 ) ; standarddev_P13=std (P13);

standarddev_P14=std (P14); standarddev_P15=std (P15); standarddev_P16=std (P $16)$; standarddev_P17=std (P17); standarddev_P18=std (P18); standarddev_P19=S td (P19); standarddev_P20=std (P20);

standarddev_P21=std (P21); standarddev_P22=std (P22) ; standarddev_P23=std (P 23 ) ; standarddev_P24=std (P24); standarddev_P25=std (P25); standarddev_P26=s td (P26); standarddev_P27=std (P27);

standarddev_P28=std (P28); standarddev_P29=std (P29); standarddev_P30=std (P $30)$; standarddev_P31=std (P31);

ocombine load cell readings from individual files into one array TopLift $=[$ TLV0; TLV1; TLV2; TLV3; TLV4; TLV5; TLV6; TLV7; TLV8; TLV9; TLV10; TLV11; TLV12; TLV13; TLV14; TLV15; TLV16; TLV1 7; TLV18; TLV19; TLV20; TLV21; TLV22; TLV23 ; TLV24; TLV25; TLV26; TLV27; TLV28; TLV29; TLV30; TLV31] ; 
TopDrag= [TDV0; TDV1; TDV2; TDV3; TDV4; TDV5; TDV6; TDV7; TDV8; TDV9; TDV10; TDV11; TDV12; TDV13; TDV14; TDV15; TDV16; TDV1 7; TDV18; TDV19; TDV20; TDV21; TDV22; TDV23 ; TDV24; TDV25; TDV26; TDV2 7; TDV28; TDV29; TDV30; TDV31] ; BotLift $=[\mathrm{BLV0}$; BLV1; BLV2;BLV3 ; BLV4;BLV5;BLV6;BLV7; BLV8; BLV9;BLV10;BLV11; BLV12;BLV13; BLV1 4;BLV15;BLV16;BLV1 7; BLV18; BLV19;BLV20;BLV21;BLV22; BLV23 ;BLV24;BLV25;BLV26;BLV2 7 BLV28;BLV29;BLV30;BLV31] ;

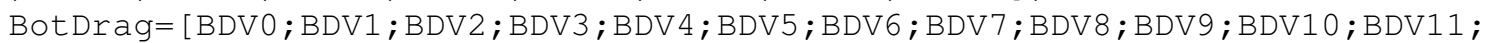
BDV1 2 ; BDV13; BDV1 4;BDV15;BDV16;BDV1 7;BDV18;BDV19;BDV20;BDV21;BDV22;BDV23 ;BDV24;BDV25;BDV26;BDV27;BDV28;BDV29;BDV30;BDV31] ;

Bottom_lift=BotLift*1807.1+259.08;

Calculate Total Lift and Drag and statistics

TotalL=TopLift+BotLift;

TotalD=TopDrag+BotDrag;

av_TL $=(0.005 *$ mean $($ TDV0 $)+0.3972) *$ mean (TopLift) ;

av_TD $=(-0.0083 *$ mean $(\mathrm{TLVO})+0.5716) *$ mean $(\mathrm{TopDrag}) ;$

av_TD2 $=(-0.0083 *$ mean $($ TLV0 $)+0.5716) *$ mean $($ TDV0 $) ;$

$\mathrm{av \_ BL}=(0.0019 *$ mean $($ BDV0 $)+0.7163) *$ mean (BotLift) ;

$\mathrm{av \_ BD}=(-0.0012 *$ mean $(\mathrm{BLV0})+0.587) *$ mean $($ BotDrag $) ;$

avg_totLift $=a v_{-}$TL $+a v_{-} B L$;

av_totalL=mean (TotalL);

av_totalD=mean (TotalD);

std_dev_TL=std (TopLift);

std_dev_TD=std ( TopDrag) ;

std_dev_TD2=std ( TDV0);

std_dev_BL=std (BotLift);

std_dev_BD=std (BotDrag);

std_dev_totalL=std ( TotalL);

std_dev_totalD=std ( TotalD);

\%Plots

[M2, N2 ] =size (TopLift);

x_chord $=1: 1:$ M2 ;

figure (1)

subplot $(2,2,1)$

plot (x_chord,TopLift); title('Top Lift

Datapoints'); xlabel('Datapoint');ylabel('Top Lift Force (lbf)');

subplot $(2,2,2)$

plot (x_chord, BotLift); title('Bottom Lift

Datapoints'); xlabel ('Datapoint'); ylabel ('Bottom Lift Force (lbf)');

subplot $(2,2,3)$

plot (x_chord, TopDrag); title('Top Drag

Datapoints'); xlabel('Datapoint');ylabel('Top Drag Force (lbf)');

subplot $(2,2,4)$

plot (x_chord, BotDrag); title('Bottom Drag

Datapoints'); xlabel ('Datapoint'); ylabel('Bottom Drag Force (lbf)');

figure (2)

plot (x_chord, TotalL,x_chord, TotalD); legend('Total Lift','Total

Drag'); title('Total Force

Datapoints'); xlabel ('Datapoint'); ylabel('Total Force (lbf)');

figure (3)

subplot $(4,4,1), \operatorname{plot}(\mathrm{x}, \mathrm{P} 0)$; title('Pressure 0

Datapoints'); xlabel ('Datapoint'); ylabel('Pressure 0 (psid)');

subplot $(4,4,2)$, plot (x,P1); title('Pressure 1

Datapoints'); xlabel ('Datapoint');ylabel('Pressure 1 (psid)');

subplot $(4,4,3)$, plot $(x, P 2)$; title ('Pressure 2

Datapoints'); xlabel('Datapoint');ylabel('Pressure 2 (psid)');

subplot $(4,4,4), \operatorname{plot}(\mathrm{x}, \mathrm{P} 3)$; title('Pressure 3

Datapoints'); xlabel ('Datapoint');ylabel('Pressure 3 (psid)'); 
subplot $(4,4,5), \operatorname{plot}(\mathrm{x}, \mathrm{P} 4)$; title('Pressure 4

Datapoints'); xlabel('Datapoint');ylabel('Pressure 4 (psid)'); subplot $(4,4,6)$, plot (x,P5); title('Pressure 5

Datapoints'); xlabel ('Datapoint');ylabel('Pressure 5 (psid)'); subplot $(4,4,7)$, plot $(x, P 6)$; title('Pressure 6

Datapoints'); xlabel('Datapoint');ylabel('Pressure 6 (psid)'); subplot $(4,4,8), \operatorname{plot}(\mathrm{x}, \mathrm{P} 7)$; title('Pressure 7

Datapoints'); xlabel ('Datapoint');ylabel('Pressure 7 (psid)'); subplot $(4,4,9)$, plot $(x, P 8)$; title('Pressure 8

Datapoints'); xlabel('Datapoint');ylabel('Pressure 8 (psid)'); subplot $(4,4,10)$, plot $(x, P 9)$; title('Pressure 9

Datapoints'); xlabel ('Datapoint');ylabel('Pressure 9 (psid)'); subplot $(4,4,11), \operatorname{plot}(\mathrm{x}, \mathrm{P} 10)$; title('Pressure 10

Datapoints'); xlabel ('Datapoint'); ylabel('Pressure 10 (psid)'); subplot $(4,4,12), \operatorname{plot}(\mathrm{x}, \mathrm{P} 11)$; title('Pressure 11 Datapoints'); xlabel ('Datapoint') ; ylabel('Pressure 11 (psid)'); subplot $(4,4,13), \operatorname{plot}(\mathrm{x}, \mathrm{P} 12)$; title('Pressure 12 Datapoints'); xlabel ('Datapoint') ;ylabel('Pressure 12 (psid)'); subplot $(4,4,14), \operatorname{plot}(\mathrm{x}, \mathrm{P} 13)$; title('Pressure 13

Datapoints'); xlabel ('Datapoint') iylabel('Pressure 13 (psid)'); subplot $(4,4,15)$, plot (x,P14); title('Pressure 14 Datapoints'); xlabel ('Datapoint'); ylabel('Pressure 14 (psid)'); subplot $(4,4,16), \operatorname{plot}(\mathrm{x}, \mathrm{P} 15)$; title('Pressure 15

Datapoints'); xlabel ('Datapoint');ylabel('Pressure 15 (psid)'); figure (4)

subplot $(4,4,1)$, plot (x,P16); title('Pressure 16

Datapoints') ; xlabel ('Datapoint');ylabel('Pressure 16 (psid)');

subplot $(4,4,2), \operatorname{plot}(\mathrm{x}, \mathrm{P} 17)$; title('Pressure 17

Datapoints'); xlabel ('Datapoint');ylabel('Pressure 17 (psid)'); subplot $(4,4,3), \operatorname{plot}(\mathrm{x}, \mathrm{P} 18)$; title('Pressure 18

Datapoints'); xlabel ('Datapoint');ylabel('Pressure 18 (psid)'); subplot $(4,4,4), \operatorname{plot}(\mathrm{x}, \mathrm{P} 19)$; title('Pressure 19

Datapoints'); xlabel ('Datapoint');ylabel('Pressure 19 (psid)'); subplot $(4,4,5)$, plot $(x, P 20)$; title('Pressure 20

Datapoints'); xlabel ('Datapoint');ylabel('Pressure 20 (psid)'); subplot $(4,4,6)$, plot $(x, P 21)$; title('Pressure 21

Datapoints'); xlabel ('Datapoint');ylabel('Pressure 21 (psid)'); subplot $(4,4,7)$, plot (x,P22); title('Pressure 22

Datapoints'); xlabel('Datapoint');ylabel('Pressure 22 (psid)'); subplot $(4,4,8), \operatorname{plot}(\mathrm{x}, \mathrm{P} 23)$; title('Pressure 23

Datapoints'); xlabel('Datapoint');ylabel('Pressure 23 (psid)'); subplot $(4,4,9)$, plot (x,P24); title('Pressure 24

Datapoints'); xlabel ('Datapoint'); ylabel('Pressure 24 (psid)'); subplot $(4,4,10), \operatorname{plot}(\mathrm{x}, \mathrm{P} 25)$; title('Pressure 25

Datapoints'); xlabel('Datapoint');ylabel('Pressure 25 (psid)'); subplot $(4,4,11)$, plot (x,P26); title('Pressure 26

Datapoints') ; xlabel ('Datapoint');ylabel('Pressure 26 (psid)'); subplot $(4,4,12)$, plot (x,P27); title('Pressure 27

Datapoints'); xlabel ('Datapoint'); ylabel('Pressure 27 (psid)'); subplot $(4,4,13), \operatorname{plot}(\mathrm{x}, \mathrm{P} 28)$; title('Pressure 28

Datapoints'); xlabel ('Datapoint'); ylabel('Pressure 28 (psid)'); subplot $(4,4,14)$, plot (x,P29); title('Pressure 29

Datapoints'); xlabel('Datapoint');ylabel('Pressure 29 (psid)'); subplot $(4,4,15)$, plot (x,P30); title('Pressure 30

Datapoints'); xlabel ('Datapoint');ylabel('Pressure 30 (psid)'); subplot $(4,4,16), \operatorname{plot}(\mathrm{x}, \mathrm{P} 31)$; title('Pressure 31

Datapoints'); xlabel ('Datapoint') ;ylabel('Pressure 31 (psid)'); 


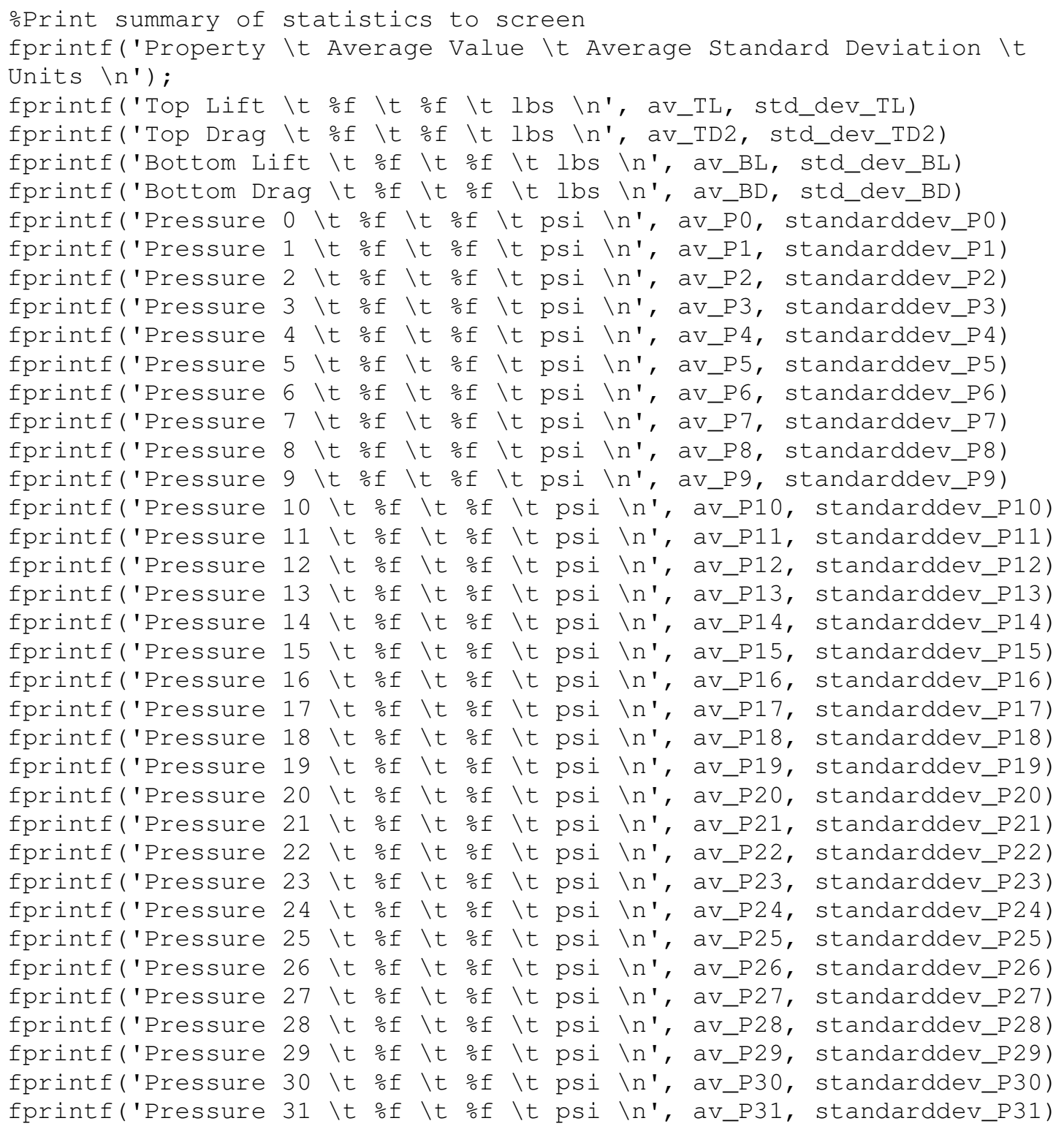




\section{Program C-3: Active Model Matlab Data Reduction Program}

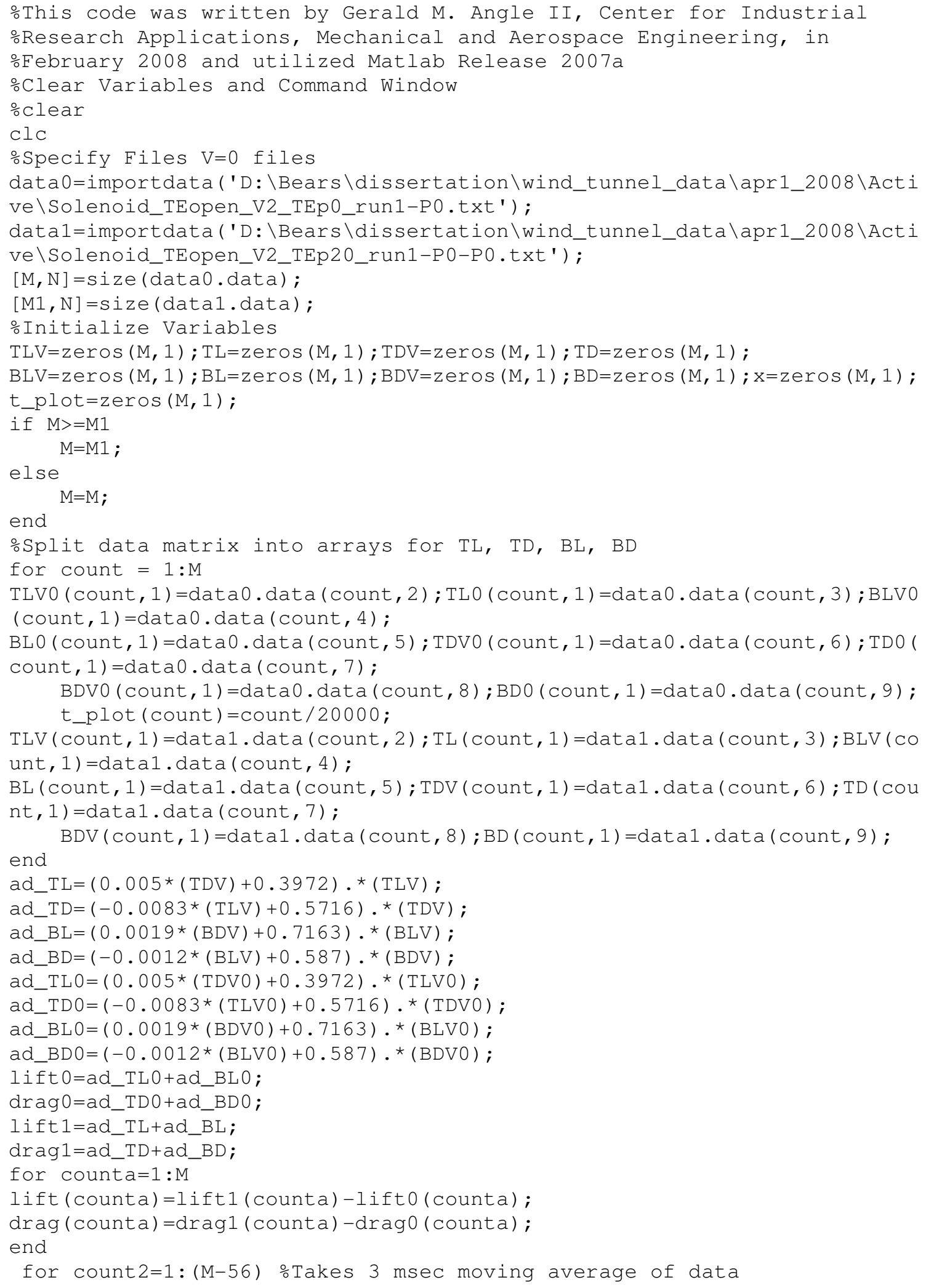


mov_avg_lift $($ count 2$)=($ lift $($ count 2$)+$ lift $($ count $2+1)+$ lift $($ count $2+2)+1$ ift $(c$ ount $2+3)+$ lift $(\operatorname{count} 2+4)+$ lift $(\operatorname{count} 2+5)+$ lift $(\operatorname{count} 2+6)+1$ ift $(\operatorname{count} 2+7)+1 i$ ft ( count $2+8)+$ lift ( count $2+9)+$ lift ( count $2+10)+$ lift ( count $2+11)+$ lift ( count 2 $+12)+$ lift $($ count $2+13)+$ lift $(\operatorname{count} 2+14)+$ lift $(\operatorname{count} 2+15)+1$ ift $(\operatorname{count} 2+16)+1 i$ ft ( count $2+17)+$ lift ( count $2+18)+$ lift (count $2+19)+$ lift (count $2+20)+$ ift (coun t $2+21)+$ lift $(\operatorname{count} 2+22)+\operatorname{lift}(\operatorname{count} 2+23)+\operatorname{lift}(\operatorname{count} 2+24)+$ ift $(\operatorname{count} 2+25)+$ lift ( count $2+26)+$ lift ( count $2+27)+$ lift ( count $2+28)+$ lift ( count $2+29)+$ ift ( co unt $2+30)+$ lift $(\operatorname{count} 2+31)+\operatorname{lift}(\operatorname{count} 2+32)+\operatorname{lift}(\operatorname{count} 2+33)+1$ ift $(\operatorname{count} 2+34$ )+ lift ( count $2+35)+$ lift ( count $2+36)+$ lift $($ count $2+37)+$ lift $(\operatorname{count} 2+38)+1$ ift ( count $2+39)+$ lift $(\operatorname{count} 2+40)+$ ift $(\operatorname{count} 2+41)+$ lift $(\operatorname{count} 2+42)+1$ ift $(\operatorname{count} 2+$ $43)+$ lift ( count $2+44)+\operatorname{lift}(\operatorname{count} 2+45)+$ lift $(\operatorname{count} 2+46)+$ lift $(\operatorname{count} 2+47)+1$ if $t(\operatorname{count} 2+48)+$ lift $(\operatorname{count} 2+49)+$ lift $($ count $2+50)+$ lift $(\operatorname{count} 2+51)+1$ ift (count $2+52)+\operatorname{lift}(\operatorname{count} 2+53)+\operatorname{lift}(\operatorname{count} 2+54)+\operatorname{lift}(\operatorname{count} 2+55)+\operatorname{lift}(\operatorname{count} 2+56)) /$ 57 ;

mov_avg_drag $($ count 2$)=($ drag $($ count 2$)+$ drag $($ count $2+1)+d r a g($ count $2+2)+$ drag $($ c ount $2+3)+$ drag $(\operatorname{count} 2+4)+\operatorname{drag}(\operatorname{count} 2+5)+\operatorname{drag}(\operatorname{count} 2+6)+\operatorname{drag}(\operatorname{count} 2+7)+d r$ $\mathrm{ag}($ count $2+8)+\mathrm{drag}(\operatorname{count} 2+9)+\mathrm{drag}($ count $2+10)+\mathrm{drag}(\operatorname{count} 2+11)+\mathrm{drag}(\operatorname{count} 2$ $+12)+d r a g(\operatorname{count} 2+13)+d r a g(\operatorname{count} 2+14)+d r a g(\operatorname{count} 2+15)+d r a g(\operatorname{count} 2+16)+d r$ $\operatorname{ag}(\operatorname{count} 2+17)+\mathrm{drag}(\operatorname{count} 2+18)+\mathrm{drag}($ count $2+19)+\mathrm{drag}(\operatorname{count} 2+20)+\mathrm{drag}(\operatorname{coun}$ t $2+21)+d r a g(\operatorname{count} 2+22)+d r a g(\operatorname{count} 2+23)+d r a g(\operatorname{count} 2+24)+d r a g(\operatorname{count} 2+25)+$ drag ( count $2+26)+$ drag $(\operatorname{count} 2+27)+$ drag $(\operatorname{count} 2+28)+d r a g(\operatorname{count} 2+29)+\operatorname{drag}(\operatorname{co}$ unt $2+30)+d r a g(\operatorname{count} 2+31)+d r a g(\operatorname{count} 2+32)+d r a g(\operatorname{count} 2+33)+\operatorname{drag}(\operatorname{count} 2+34$ )$+\operatorname{drag}(\operatorname{count} 2+35)+\operatorname{drag}(\operatorname{count} 2+36)+\operatorname{drag}(\operatorname{count} 2+37)+\operatorname{drag}(\operatorname{count} 2+38)+\mathrm{drag}($ count $2+39)+$ drag $(\operatorname{count} 2+40)+d r a g(\operatorname{count} 2+41)+d r a g(\operatorname{count} 2+42)+\operatorname{drag}(\operatorname{count} 2+$ $43)+\operatorname{drag}(\operatorname{count} 2+44)+\operatorname{drag}(\operatorname{count} 2+45)+\operatorname{drag}(\operatorname{count} 2+46)+\operatorname{drag}(\operatorname{count} 2+47)+\mathrm{dra}$ $g(\operatorname{count} 2+48)+d r a g(\operatorname{count} 2+49)+d r a g(\operatorname{count} 2+50)+\operatorname{drag}(\operatorname{count} 2+51)+\mathrm{drag}(\operatorname{count}$ $2+52)+\operatorname{drag}(\operatorname{count} 2+53)+\operatorname{drag}(\operatorname{count} 2+54)+\operatorname{drag}(\operatorname{count} 2+55)+\operatorname{drag}(\operatorname{count} 2+56)) /$ 57 ;

end

$t \_m($ count 2$)=t \_p l o t(\operatorname{count} 2+\operatorname{ceil}(57 / 2)) ;$

figure (1)

plot (t_m, mov_avg_lift);

xlabel('Time (sec)'); ylabel('Lift Force (lbf)');

axis ([0,0.5, min (mov_avg_lift), max (mov_avg_lift)]);

figure (2)

plot (t_m,mov_avg_drag);

xlabel('Time (sec)'); ylabel('Drag Force (lbf)');

axis ([0,0.5, min (mov_avg_drag), max (mov_avg_drag)]); 


\section{Appendix}

D

Lift Response to Solenoid Activation Figures 


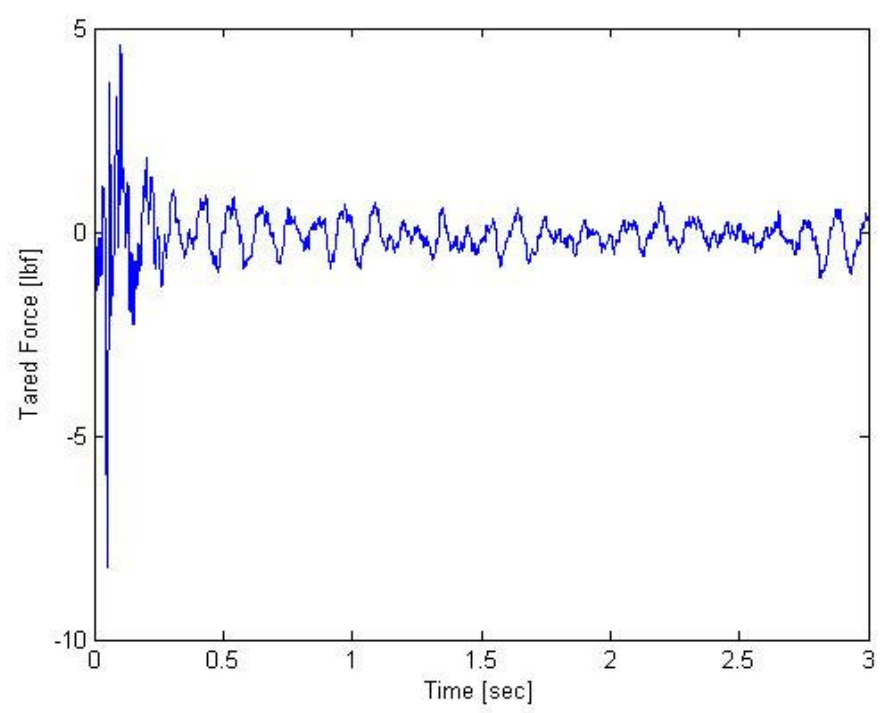

Figure D. 1: Experimental time response characteristics for lift with the $30 \mathrm{psig}$ regulated trailing edge opening test condition at 0.7 degrees angle of attack with an $82 \mathrm{ft} / \mathrm{s}$ freestream velocity

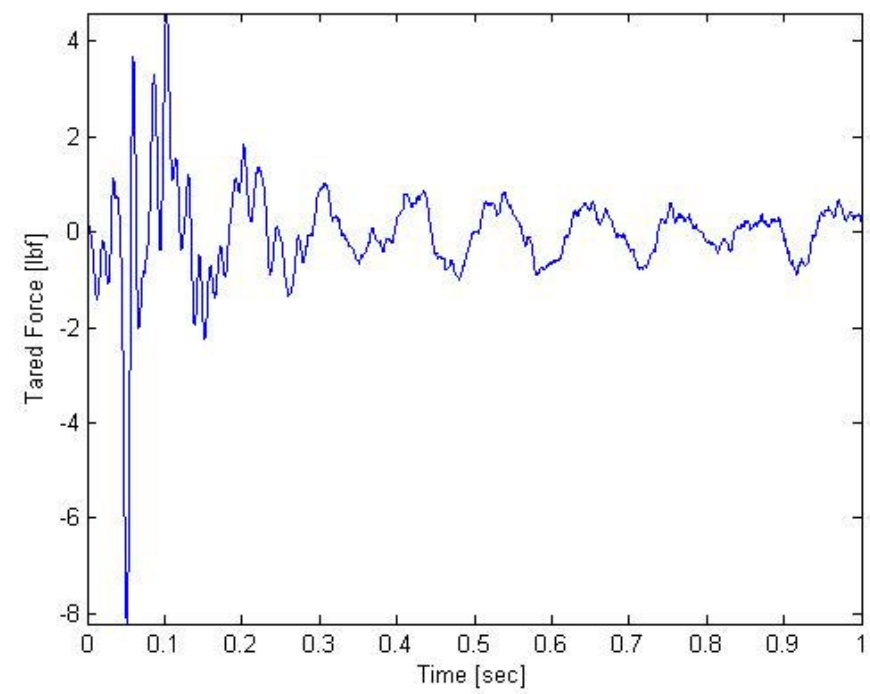

Figure D. 2: Lift force for one second time axis view for experimental time response characteristics with the $30 \mathrm{psig}$ regulated trailing edge opening test condition at 0.7 degrees angle of attack with an $82 \mathrm{ft} / \mathrm{s}$ freestream velocity 


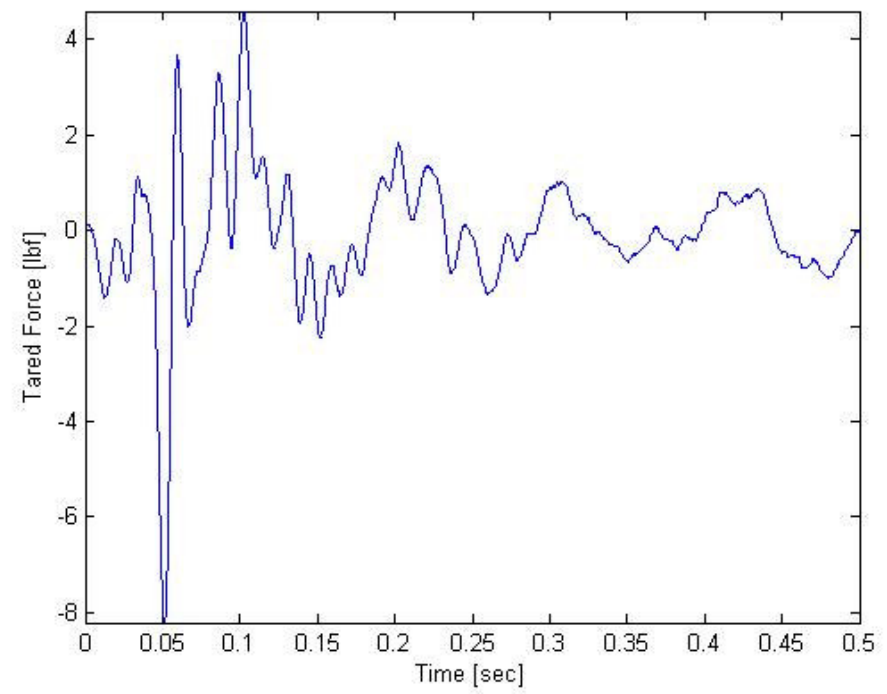

Figure D. 3: Lift force for one-half second time axis view for experimental time response characteristics with the 30 psig regulated trailing edge opening test condition at 0.7 degrees angle of attack with an $82 \mathrm{ft} / \mathrm{s}$ freestream velocity 


\section{Appendix}

$\mathbf{E}$

Baseline Model Data Tables 
Table E. 1: Lift and drag coefficients for the baseline model at various angles of attack

\begin{tabular}{|c|c|c|c|c|c|c|c|c|c|c|c|c|c|c|}
\hline $\begin{array}{c}\text { Angle of } \\
\text { Attack }\end{array}$ & \multicolumn{2}{|c|}{ Run 1} & \multicolumn{2}{|c|}{ Run 2} & \multicolumn{2}{|c|}{ Run 3} & \multicolumn{2}{|c|}{ Run 4} & \multicolumn{2}{|c|}{ Run 5} & \multicolumn{2}{|c|}{ Run 6} & \multicolumn{2}{|c|}{ Run 7} \\
\hline deg & $\mathrm{Cl}$ & $\mathrm{Cd}$ & $\mathrm{Cl}$ & $\mathrm{Cd}$ & $\mathrm{Cl}$ & $\mathrm{Cd}$ & $\mathrm{Cl}$ & $\mathrm{Cd}$ & $\mathrm{Cl}$ & $\mathrm{Cd}$ & $\mathrm{Cl}$ & $\mathrm{Cd}$ & $\mathrm{Cl}$ & $\mathrm{Cd}$ \\
\hline-2.39 & -0.107 & -0.027 & -0.088 & 0.176 & -0.084 & 0.174 & -0.072 & 0.205 & -0.008 & 0.009 & -0.102 & -0.141 & -0.084 & -0.130 \\
\hline-0.14 & 0.041 & -0.082 & 0.085 & 0.051 & 0.112 & -0.107 & 0.188 & -0.059 & -0.020 & 0.012 & 0.021 & -0.014 & 0.135 & 0.117 \\
\hline 2.49 & 0.251 & -0.091 & 0.253 & 0.138 & 0.300 & -0.044 & 0.381 & -0.141 & 0.212 & -0.042 & 0.334 & 0.274 & 0.432 & 0.402 \\
\hline 3.73 & 0.415 & -0.038 & 0.551 & -0.125 & 0.566 & -0.220 & 0.586 & -0.179 & 0.462 & -0.107 & 0.561 & 0.502 & 0.639 & 0.588 \\
\hline 5.27 & 0.600 & 0.008 & 0.779 & -0.127 & 0.836 & -0.431 & 0.822 & -0.281 & 0.695 & -0.117 & 0.774 & 0.681 & 0.918 & 0.854 \\
\hline 8.16 & 0.678 & 0.076 & 0.936 & -0.186 & 0.999 & -0.491 & 0.949 & -0.281 & 0.921 & -0.138 & 0.949 & 0.794 & 1.119 & 1.025 \\
\hline 9.7 & 0.769 & 0.011 & 0.830 & -0.062 & 0.870 & -0.334 & 0.731 & 0.119 & 1.130 & -0.252 & 0.993 & 0.831 & 0.988 & 0.860 \\
\hline 11.65 & 0.683 & -0.045 & 0.767 & -0.153 & 0.757 & -0.209 & 0.620 & 0.065 & 0.968 & -0.216 & 0.876 & 0.747 & 0.815 & 0.719 \\
\hline 13.7 & 0.541 & 0.083 & 0.716 & -0.202 & 0.632 & -0.100 & 0.674 & -0.182 & 0.759 & -0.141 & 0.739 & 0.645 & 0.766 & 0.695 \\
\hline 15.38 & 0.620 & 0.026 & 0.741 & -0.325 & 0.590 & -0.077 & 0.737 & -0.183 & 0.784 & -0.133 & 0.747 & 0.622 & 0.803 & 0.708 \\
\hline 17.47 & 0.639 & 0.012 & 0.690 & -0.177 & 0.600 & -0.086 & 0.756 & -0.201 & 0.832 & -0.146 & 0.780 & 0.642 & 0.857 & 0.749 \\
\hline
\end{tabular}

Table E. 2: Lift and drag coefficients for the baseline model at various angles of attack (cont.)

\begin{tabular}{|c|c|c|c|c|c|c|c|c|c|c|c|c|}
\hline \multirow{2}{*}{$\begin{array}{c}\text { Angle of } \\
\text { Attack } \\
\text { deg }\end{array}$} & \multicolumn{2}{|c|}{ Run 8} & \multicolumn{2}{|c|}{ Run 9} & \multicolumn{2}{|c|}{ Run 10} & \multicolumn{2}{|c|}{ Run 11} & \multicolumn{2}{|c|}{ Run 12} & \multicolumn{2}{|c|}{ Run 13} \\
\hline & $\mathrm{Cl}$ & $\mathrm{Cd}$ & $\mathrm{Cl}$ & $\mathrm{Cd}$ & $\mathrm{Cl}$ & $\mathrm{Cd}$ & $\mathrm{Cl}$ & $\mathrm{Cd}$ & $\mathrm{Cl}$ & $\mathrm{Cd}$ & $\mathrm{Cl}$ & $\mathrm{Cd}$ \\
\hline-2.39 & -0.015 & -0.024 & -0.090 & -0.086 & -0.083 & 0.080 & -0.104 & 0.019 & -0.084 & -0.002 & -0.151 & -0.079 \\
\hline-0.14 & 0.091 & 0.077 & 0.065 & 0.059 & 0.070 & -0.013 & 0.063 & 0.025 & 0.082 & -0.048 & -0.026 & -0.058 \\
\hline 2.49 & 0.302 & 0.262 & 0.340 & 0.330 & 0.345 & -0.095 & 0.337 & -0.064 & 0.300 & -0.078 & 0.098 & -0.059 \\
\hline 3.73 & 0.568 & 0.526 & 0.590 & 0.561 & 0.086 & -0.034 & 0.576 & -0.110 & 0.529 & -0.100 & 0.350 & -0.119 \\
\hline 5.27 & 0.734 & 0.644 & 0.856 & 0.810 & 0.861 & -0.228 & 0.807 & -0.137 & 0.880 & -0.191 & 0.645 & -0.125 \\
\hline 8.16 & 1.070 & 0.959 & 1.043 & 0.972 & 1.095 & -0.264 & 1.059 & -0.225 & 1.038 & -0.214 & 0.905 & -0.180 \\
\hline 9.7 & 0.972 & 0.845 & 0.866 & 0.819 & 0.991 & -0.208 & 1.047 & -0.182 & 0.778 & -0.162 & 1.010 & -0.224 \\
\hline 11.65 & 0.818 & 0.723 & 0.815 & 0.759 & 0.793 & -0.136 & 0.888 & -0.174 & 0.897 & -0.191 & 0.987 & -0.231 \\
\hline 13.7 & 0.724 & 0.635 & 0.753 & 0.611 & 0.676 & -0.161 & 0.758 & -0.136 & 0.767 & -0.164 & 0.851 & -0.213 \\
\hline 15.38 & 0.866 & 0.788 & 0.785 & 0.729 & 0.792 & -0.155 & 0.813 & -0.166 & 0.836 & -0.187 & 0.772 & -0.170 \\
\hline 17.47 & 0.907 & 0.829 & 0.854 & 0.792 & 0.829 & -0.170 & 0.892 & -0.191 & 0.885 & -0.202 & 0.863 & -0.204 \\
\hline
\end{tabular}


Table E. 3: Baseline force data for Run 1, recorded at $10 \mathrm{kHz}$

\begin{tabular}{|c|c|c|c|c|c|c|c|c|c|c|c|c|}
\hline Actual AoA & Indicated AoA & Lift & Drag & \multicolumn{2}{|c|}{$\mathrm{P}$ atm } & T_tunnel & density & \multicolumn{2}{|c|}{$\Delta \mathrm{P}$} & $\mathrm{V}$ & $\mathrm{Cl}$ & $\mathrm{Cd}$ \\
\hline deg & deg & Ibs & lbs & $\mathrm{inHg}$ & psf & $\operatorname{deg} \mathrm{F}$ & slug $/ \mathrm{ft}^{\wedge} 3$ & $\mathrm{inH} 2 \mathrm{O}$ & psf & $\mathrm{ft} / \mathrm{s}$ & - & - \\
\hline-2.39 & -4 & -3.30 & -0.84 & 28.85 & 2040.46 & 60 & 0.002287 & 2.287 & 11.898 & 102.0 & -0.107 & -0.027 \\
\hline-0.14 & -2 & 1.26 & -2.52 & 28.85 & 2040.46 & 62 & 0.002278 & 2.281 & 11.867 & 102.1 & 0.041 & -0.082 \\
\hline 2.49 & 0 & 7.71 & -2.79 & 28.85 & 2040.46 & 64 & 0.002269 & 2.285 & 11.887 & 102.4 & 0.251 & -0.091 \\
\hline 3.73 & 2 & 12.72 & -1.15 & 28.85 & 2040.46 & 66 & 0.002261 & 2.282 & 11.872 & 102.5 & 0.415 & -0.038 \\
\hline 5.27 & 4 & 18.39 & 0.25 & 28.85 & 2040.46 & 68 & 0.002252 & 2.284 & 11.882 & 102.7 & 0.600 & 0.008 \\
\hline 8.16 & 6 & 20.80 & 2.35 & 28.85 & 2040.46 & 70 & 0.002244 & 2.285 & 11.887 & 102.9 & 0.678 & 0.076 \\
\hline 9.7 & 8 & 23.22 & 0.35 & 28.85 & 2040.46 & 70 & 0.002244 & 2.25 & 11.705 & 102.1 & 0.769 & 0.011 \\
\hline 11.65 & 10 & 20.74 & -1.37 & 28.85 & 2040.46 & 72 & 0.002235 & 2.263 & 11.773 & 102.6 & 0.683 & -0.045 \\
\hline 13.7 & 12 & 16.56 & 2.55 & 28.85 & 2040.46 & 73 & 0.002231 & 2.277 & 11.846 & 103.1 & 0.541 & 0.083 \\
\hline 15.38 & 14 & 18.84 & 0.78 & 28.85 & 2040.46 & 74 & 0.002227 & 2.264 & 11.778 & 102.9 & 0.620 & 0.026 \\
\hline 17.47 & 16 & 19.19 & 0.35 & 28.85 & 2040.46 & 74 & 0.002227 & 2.235 & 11.627 & 102.2 & 0.639 & 0.012 \\
\hline
\end{tabular}

Table E. 4: Baseline force data for Run 1-A, recorded at $20 \mathrm{kHz}$

\begin{tabular}{|c|c|c|c|c|c|c|c|c|c|c|c|c|}
\hline Actual AoA & Indicated AoA & Lift & Drag & \multicolumn{2}{|c|}{ P atm } & $T_{\text {_tunnel }}$ & density & \multicolumn{2}{|c|}{$\Delta \mathrm{P}$} & V & $\mathrm{Cl}$ & $\mathrm{Cd}$ \\
\hline deg & deg & Ibs & Ibs & $\mathrm{inHg}$ & psf & $\operatorname{deg} \mathrm{F}$ & slug/ft^3 & $\mathrm{inH} 2 \mathrm{O}$ & psf & $\mathrm{ft} / \mathrm{s}$ & - & - \\
\hline-2.39 & -4 & -3.11 & 2.86 & 28.85 & 2040.46 & 76 & 0.002218 & 2.607 & 13.563 & 110.6 & -0.089 & 0.082 \\
\hline-0.14 & -2 & 2.40 & -0.76 & 28.85 & 2040.46 & 76 & 0.002218 & 2.607 & 13.563 & 110.6 & 0.069 & -0.022 \\
\hline 2.49 & 0 & 10.44 & -2.87 & 28.85 & 2040.46 & 76 & 0.002218 & 2.539 & 13.209 & 109.1 & 0.306 & -0.084 \\
\hline 3.73 & 2 & 16.42 & -2.70 & 28.85 & 2040.46 & 75 & 0.002223 & 2.484 & 12.923 & 107.8 & 0.492 & -0.081 \\
\hline 5.27 & 4 & 23.24 & -4.67 & 28.85 & 2040.46 & 74 & 0.002227 & 2.422 & 12.6 & 106.4 & 0.715 & -0.143 \\
\hline
\end{tabular}


Table E. 5: Baseline force data for Run 2

\begin{tabular}{|c|c|c|c|c|c|c|c|c|c|c|c|c|}
\hline Actual AoA & Indicated AoA & Lift & Drag & \multicolumn{2}{|c|}{$\mathrm{P}$ atm } & T_tunnel & density & \multicolumn{2}{|c|}{$\Delta \mathrm{P}$} & $\mathrm{V}$ & $\mathrm{Cl}$ & $\mathrm{Cd}$ \\
\hline deg & deg & lbs & Ibs & $\mathrm{inHg}$ & psf & $\operatorname{deg} \mathrm{F}$ & slug. $\mathrm{ft}^{\wedge} 3$ & $\mathrm{inH} 2 \mathrm{O}$ & psf & $\mathrm{ft} / \mathrm{s}$ & - & - \\
\hline-2.39 & -4 & -2.90 & 5.84 & 28.92 & 2045.41 & 75 & 0.002228 & 2.466 & 12.829 & 107.3 & -0.088 & 0.176 \\
\hline-0.14 & -2 & 2.84 & 1.70 & 28.92 & 2045.41 & 74 & 0.002232 & 2.485 & 12.928 & 107.6 & 0.085 & 0.051 \\
\hline 2.49 & 0 & 8.43 & 4.60 & 28.92 & 2045.41 & 74 & 0.002232 & 2.485 & 12.928 & 107.6 & 0.253 & 0.138 \\
\hline 3.73 & 2 & 18.39 & -4.17 & 28.92 & 2045.41 & 74 & 0.002232 & 2.485 & 12.928 & 107.6 & 0.551 & -0.125 \\
\hline 5.27 & 4 & 24.37 & -3.97 & 28.92 & 2045.41 & 73 & 0.002236 & 2.33 & 12.121 & 104.1 & 0.779 & -0.127 \\
\hline 8.16 & 6 & 28.93 & -5.76 & 28.92 & 2045.41 & 72 & 0.002241 & 2.302 & 11.976 & 103.4 & 0.936 & -0.186 \\
\hline 9.7 & 8 & 25.43 & -1.90 & 28.92 & 2045.41 & 71 & 0.002245 & 2.281 & 11.867 & 102.8 & 0.830 & -0.062 \\
\hline 11.65 & 10 & 23.50 & -4.68 & 28.92 & 2045.41 & 70 & 0.002249 & 2.281 & 11.867 & 102.7 & 0.767 & -0.153 \\
\hline 13.7 & 12 & 21.92 & -6.18 & 28.92 & 2045.41 & 70 & 0.002249 & 2.281 & 11.867 & 102.7 & 0.716 & -0.202 \\
\hline 15.38 & 14 & 22.69 & -9.95 & 28.92 & 2045.41 & 70 & 0.002249 & 2.281 & 11.867 & 102.7 & 0.741 & -0.325 \\
\hline 17.47 & 16 & 21.14 & -5.42 & 28.92 & 2045.41 & 68 & 0.002258 & 2.281 & 11.867 & 102.5 & 0.690 & -0.177 \\
\hline
\end{tabular}

Table E. 6: Baseline force data for Run 3

\begin{tabular}{|c|c|c|c|c|c|c|c|c|c|c|c|c|}
\hline Actual AoA & Indicated AoA & Lift & Drag & \multicolumn{2}{|c|}{$P$ atm } & T_tunnel & density & \multicolumn{2}{|c|}{$\Delta \mathrm{P}$} & $\mathrm{V}$ & $\mathrm{Cl}$ & $\mathrm{Cd}$ \\
\hline deg & deg & lbs & lbs & $\mathrm{inHg}$ & psf & $\operatorname{deg} F$ & slug. $\mathrm{ft}^{\wedge} 3$ & inH2O & psf & $\mathrm{ft} / \mathrm{s}$ & - & - \\
\hline-2.39 & -4 & -2.82 & 5.80 & 28.92 & 2045.41 & 75 & 0.002228 & 2.485 & 12.928 & 107.7 & -0.084 & 0.174 \\
\hline-0.14 & -2 & 3.70 & -3.54 & 28.92 & 2045.41 & 76 & 0.002224 & 2.465 & 12.824 & 107.4 & 0.112 & -0.107 \\
\hline 2.49 & 0 & 9.24 & -1.34 & 28.92 & 2045.41 & 76 & 0.002224 & 2.291 & 11.919 & 103.5 & 0.300 & -0.044 \\
\hline 3.73 & 2 & 17.35 & -6.74 & 28.92 & 2045.41 & 77 & 0.00222 & 2.284 & 11.882 & 103.5 & 0.566 & -0.220 \\
\hline 5.27 & 4 & 25.97 & -13.40 & 28.92 & 2045.41 & 77 & 0.00222 & 2.314 & 12.038 & 104.1 & 0.836 & -0.431 \\
\hline 8.16 & 6 & 31.03 & -15.25 & 28.92 & 2045.41 & 77 & 0.00222 & 2.314 & 12.038 & 104.1 & 0.999 & -0.491 \\
\hline 9.7 & 8 & 27.03 & -10.39 & 28.92 & 2045.41 & 77 & 0.00222 & 2.314 & 12.038 & 104.1 & 0.870 & -0.334 \\
\hline 11.65 & 10 & 23.53 & -6.50 & 28.92 & 2045.41 & 77 & 0.00222 & 2.314 & 12.038 & 104.1 & 0.757 & -0.209 \\
\hline 13.7 & 12 & 19.49 & -3.09 & 28.92 & 2045.41 & 76 & 0.002224 & 2.295 & 11.939 & 103.6 & 0.632 & -0.100 \\
\hline 15.38 & 14 & 19.51 & -2.56 & 28.92 & 2045.41 & 76 & 0.002224 & 2.465 & 12.824 & 107.4 & 0.590 & -0.077 \\
\hline 17.47 & 16 & 20.04 & -2.87 & 28.92 & 2045.41 & 75 & 0.002228 & 2.485 & 12.928 & 107.7 & 0.600 & -0.086 \\
\hline
\end{tabular}


Table E. 7: Baseline force data for Run 4

\begin{tabular}{|c|c|c|c|c|c|c|c|c|c|c|c|c|}
\hline Actual AoA & Indicated AoA & Lift & Drag & \multicolumn{2}{|c|}{ P_atm } & T_tunnel & density & \multicolumn{2}{|c|}{$\Delta \mathrm{P}$} & $\mathrm{V}$ & $\mathrm{Cl}$ & $\mathrm{Cd}$ \\
\hline deg & deg & Ibs & lbs & inHg & psf & $\operatorname{deg} F$ & slug. $\mathrm{ft}^{\wedge} 3$ & inH2O & psf & $\mathrm{ft} / \mathrm{s}$ & - & - \\
\hline-2.39 & -4 & -2.23 & 6.36 & 29.31 & 2073 & 53 & 0.002355 & 2.307 & 12.002 & 101.0 & -0.072 & 0.205 \\
\hline-0.14 & -2 & 5.78 & -1.81 & 29.31 & 2073 & 60 & 0.002323 & 2.284 & 11.882 & 101.1 & 0.188 & -0.059 \\
\hline 2.49 & 0 & 12.81 & -4.75 & 29.31 & 2073 & 64 & 0.002305 & 2.505 & 13.032 & 106.3 & 0.381 & -0.141 \\
\hline 3.73 & 2 & 19.28 & -5.88 & 29.31 & 2073 & 67 & 0.002292 & 2.449 & 12.741 & 105.4 & 0.586 & -0.179 \\
\hline 5.27 & 4 & 25.66 & -8.78 & 29.31 & 2073 & 69 & 0.002284 & 2.325 & 12.095 & 102.9 & 0.822 & -0.281 \\
\hline 8.16 & 6 & 28.94 & -8.57 & 29.31 & 2073 & 70 & 0.002279 & 2.272 & 11.82 & 101.8 & 0.949 & -0.281 \\
\hline 9.7 & 8 & 22.26 & 3.63 & 29.31 & 2073 & 60 & 0.002323 & 2.266 & 11.789 & 100.7 & 0.731 & 0.119 \\
\hline 11.65 & 10 & 19.05 & 1.99 & 29.31 & 2073 & 62 & 0.002314 & 2.287 & 11.898 & 101.4 & 0.620 & 0.065 \\
\hline 13.7 & 12 & 20.49 & -5.54 & 29.31 & 2073 & 65 & 0.002301 & 2.265 & 11.783 & 101.2 & 0.674 & -0.182 \\
\hline 15.38 & 14 & 21.81 & -5.42 & 29.31 & 2073 & 68 & 0.002288 & 2.202 & 11.456 & 100.1 & 0.737 & -0.183 \\
\hline 17.47 & 16 & 22.00 & -5.86 & 29.31 & 2073 & 70 & 0.002279 & 2.168 & 11.279 & 99.5 & 0.756 & -0.201 \\
\hline
\end{tabular}

Table E. 8: Baseline force data for Run 5

\begin{tabular}{|c|c|c|c|c|c|c|c|c|c|c|c|c|}
\hline Actual AoA & Indicated AoA & Lift & Drag & \multicolumn{2}{|c|}{$\mathrm{P}$ _atm } & T_tunnel & density & \multicolumn{2}{|c|}{$\Delta \mathrm{P}$} & V & $\mathrm{Cl}$ & $\mathrm{Cd}$ \\
\hline deg & deg & lbs & lbs & inHg & psf & $\operatorname{deg} F$ & slug.ft^3 & inH2O & psf & $\mathrm{ft} / \mathrm{s}$ & - & - \\
\hline-2.39 & -4 & -0.24 & 0.27 & 29.2 & 2065.22 & 65 & 0.002292 & 2.275 & 11.835 & 101.6 & -0.008 & 0.009 \\
\hline-0.14 & -2 & -0.69 & 0.42 & 29.2 & 2065.22 & 69 & 0.002275 & 2.544 & 13.235 & 107.9 & -0.020 & 0.012 \\
\hline 2.49 & 0 & 7.12 & -1.40 & 29.2 & 2065.22 & 71 & 0.002266 & 2.5 & 13.006 & 107.1 & 0.212 & -0.042 \\
\hline 3.73 & 2 & 15.20 & -3.52 & 29.2 & 2065.22 & 72 & 0.002262 & 2.451 & 12.751 & 106.2 & 0.462 & -0.107 \\
\hline 5.27 & 4 & 22.07 & -3.72 & 29.2 & 2065.22 & 73 & 0.002258 & 2.365 & 12.304 & 104.4 & 0.695 & -0.117 \\
\hline 8.16 & 6 & 28.37 & -4.25 & 29.2 & 2065.22 & 74 & 0.002254 & 2.295 & 11.939 & 102.9 & 0.921 & -0.138 \\
\hline 9.7 & 8 & 34.58 & -7.72 & 29.2 & 2065.22 & 66 & 0.002288 & 2.28 & 11.861 & 101.8 & 1.130 & -0.252 \\
\hline 11.65 & 10 & 29.00 & -6.46 & 29.2 & 2065.22 & 70 & 0.002271 & 2.231 & 11.606 & 101.1 & 0.968 & -0.216 \\
\hline 13.7 & 12 & 22.62 & -4.19 & 29.2 & 2065.22 & 71 & 0.002266 & 2.22 & 11.549 & 101.0 & 0.759 & -0.141 \\
\hline 15.38 & 14 & 23.03 & -3.89 & 29.2 & 2065.22 & 72 & 0.002262 & 2.188 & 11.383 & 100.3 & 0.784 & -0.133 \\
\hline 17.47 & 16 & 24.07 & -4.21 & 29.2 & 2065.22 & 73 & 0.002258 & 2.153 & 11.201 & 99.6 & 0.832 & -0.146 \\
\hline
\end{tabular}


Table E. 9: Baseline force data for Run 6

\begin{tabular}{|c|c|c|c|c|c|c|c|c|c|c|c|c|}
\hline Actual AoA & Indicated AoA & Lift & Drag & \multicolumn{2}{|c|}{$\mathrm{P}$ atm } & T_tunnel & density & \multicolumn{2}{|c|}{$\Delta \mathrm{P}$} & $\mathrm{V}$ & $\mathrm{Cl}$ & $\mathrm{Cd}$ \\
\hline deg & deg & Ibs & lbs & $\mathrm{inHg}$ & psf & $\operatorname{deg} \mathrm{F}$ & slug. $\mathrm{ft}^{\wedge} 3$ & $\mathrm{inH} 2 \mathrm{O}$ & psf & $\mathrm{ft} / \mathrm{s}$ & - & - \\
\hline-2.39 & -4 & -3.39 & -4.68 & 29.19 & 2064.51 & 75 & 0.002249 & 2.471 & 12.855 & 106.9 & -0.102 & -0.141 \\
\hline-0.14 & -2 & 0.69 & -0.49 & 29.19 & 2064.51 & 76 & 0.002245 & 2.5 & 13.006 & 107.7 & 0.021 & -0.014 \\
\hline 2.49 & 0 & 10.86 & 8.90 & 29.19 & 2064.51 & 76 & 0.002245 & 2.42 & 12.59 & 105.9 & 0.334 & 0.274 \\
\hline 3.73 & 2 & 17.88 & 16.00 & 29.19 & 2064.51 & 77 & 0.00224 & 2.375 & 12.356 & 105.0 & 0.561 & 0.502 \\
\hline 5.27 & 4 & 24.22 & 21.31 & 29.19 & 2064.51 & 78 & 0.002236 & 2.33 & 12.121 & 104.1 & 0.774 & 0.681 \\
\hline 8.16 & 6 & 28.98 & 24.23 & 29.19 & 2064.51 & 78 & 0.002236 & 2.273 & 11.825 & 102.8 & 0.949 & 0.794 \\
\hline 9.7 & 8 & 29.34 & 24.56 & 29.19 & 2064.51 & 78 & 0.002236 & 2.2 & 11.445 & 101.2 & 0.993 & 0.831 \\
\hline 11.65 & 10 & 25.60 & 21.83 & 29.19 & 2064.51 & 78 & 0.002236 & 2.175 & 11.315 & 100.6 & 0.876 & 0.747 \\
\hline 13.7 & 12 & 21.62 & 18.87 & 29.19 & 2064.51 & 77 & 0.00224 & 2.18 & 11.341 & 100.6 & 0.739 & 0.645 \\
\hline 15.38 & 14 & 21.67 & 18.03 & 29.19 & 2064.51 & 76 & 0.002245 & 2.16 & 11.237 & 100.1 & 0.747 & 0.622 \\
\hline 17.47 & 16 & 22.42 & 18.46 & 29.19 & 2064.51 & 76 & 0.002245 & 2.142 & 11.143 & 99.6 & 0.780 & 0.642 \\
\hline
\end{tabular}

Table E. 10: Baseline force data for Run 7

\begin{tabular}{|c|c|c|c|c|c|c|c|c|c|c|c|c|}
\hline Actual AoA & Indicated AoA & Lift & Drag & \multicolumn{2}{|c|}{$\mathrm{P}_{\text {atm }}$} & T_tunnel & density & \multicolumn{2}{|c|}{$\Delta \mathrm{P}$} & V & $\mathrm{Cl}$ & $\mathrm{Cd}$ \\
\hline deg & deg & lbs & lbs & inHg & psf & $\operatorname{deg} F$ & slug.ft^3 & in $\mathrm{H} 2 \mathrm{O}$ & psf & $\mathrm{ft} / \mathrm{s}$ & - & - \\
\hline-2.39 & -4 & -2.58 & -3.96 & 28.84 & 2039.75 & 62 & 0.002277 & 2.276 & 11.841 & 102.0 & -0.084 & -0.130 \\
\hline-0.14 & -2 & 4.11 & 3.55 & 28.84 & 2039.75 & 67 & 0.002256 & 2.266 & 11.789 & 102.2 & 0.135 & 0.117 \\
\hline 2.49 & 0 & 12.89 & 12.01 & 28.84 & 2039.75 & 70 & 0.002243 & 2.222 & 11.56 & 101.5 & 0.432 & 0.402 \\
\hline 3.73 & 2 & 18.59 & 17.10 & 28.84 & 2039.75 & 71 & 0.002239 & 2.165 & 11.263 & 100.3 & 0.639 & 0.588 \\
\hline 5.27 & 4 & 26.02 & 24.20 & 28.84 & 2039.75 & 72 & 0.002234 & 2.11 & 10.977 & 99.1 & 0.918 & 0.854 \\
\hline 8.16 & 6 & 30.37 & 27.84 & 28.84 & 2039.75 & 73 & 0.00223 & 2.022 & 10.519 & 97.1 & 1.119 & 1.025 \\
\hline 9.7 & 8 & 27.15 & 23.62 & 28.84 & 2039.75 & 64 & 0.002268 & 2.046 & 10.644 & 96.9 & 0.988 & 0.860 \\
\hline 11.65 & 10 & 21.89 & 19.31 & 28.84 & 2039.75 & 68 & 0.002251 & 2 & 10.405 & 96.1 & 0.815 & 0.719 \\
\hline 13.7 & 12 & 20.37 & 18.48 & 28.84 & 2039.75 & 70 & 0.002243 & 1.981 & 10.306 & 95.9 & 0.766 & 0.695 \\
\hline 15.38 & 14 & 21.04 & 18.57 & 28.84 & 2039.75 & 72 & 0.002234 & 1.952 & 10.155 & 95.3 & 0.803 & 0.708 \\
\hline 17.47 & 16 & 22.15 & 19.36 & 28.84 & 2039.75 & 73 & 0.00223 & 1.924 & 10.009 & 94.7 & 0.857 & 0.749 \\
\hline
\end{tabular}


Table E. 11: Baseline force data for Run 8

\begin{tabular}{|c|c|c|c|c|c|c|c|c|c|c|c|c|}
\hline Actual AoA & Indicated AoA & Lift & Drag & \multicolumn{2}{|c|}{$P$ _atm } & T_tunnel & density & \multicolumn{2}{|c|}{$\Delta \mathrm{P}$} & $\mathrm{V}$ & $\mathrm{Cl}$ & $\mathrm{Cd}$ \\
\hline deg & deg & Ibs & Ibs & $\mathrm{inHg}$ & psf & $\operatorname{deg} \mathrm{F}$ & slug. $\mathrm{ft}^{\wedge} 3$ & $\mathrm{inH} 2 \mathrm{O}$ & psf & $\mathrm{ft} / \mathrm{s}$ & - & - \\
\hline-2.39 & -4 & -0.46 & -0.73 & 28.75 & 2033.39 & 70 & 0.002236 & 2.275 & 11.835 & 102.9 & -0.015 & -0.024 \\
\hline-0.14 & -2 & 2.79 & 2.35 & 28.75 & 2033.39 & 70 & 0.002236 & 2.273 & 11.825 & 102.8 & 0.091 & 0.077 \\
\hline 2.49 & 0 & 9.26 & 8.04 & 28.75 & 2033.39 & 71 & 0.002232 & 2.281 & 11.867 & 103.1 & 0.302 & 0.262 \\
\hline 3.73 & 2 & 16.60 & 15.36 & 28.75 & 2033.39 & 71 & 0.002232 & 2.175 & 11.315 & 100.7 & 0.568 & 0.526 \\
\hline 5.27 & 4 & 21.34 & 18.72 & 28.75 & 2033.39 & 72 & 0.002227 & 2.165 & 11.263 & 100.6 & 0.734 & 0.644 \\
\hline 8.16 & 6 & 32.55 & 29.16 & 28.75 & 2033.39 & 78 & 0.002203 & 2.265 & 11.783 & 103.4 & 1.070 & 0.959 \\
\hline 9.7 & 8 & 29.39 & 25.54 & 28.75 & 2033.39 & 73 & 0.002223 & 2.251 & 11.711 & 102.6 & 0.972 & 0.845 \\
\hline 11.65 & 10 & 24.70 & 21.82 & 28.75 & 2033.39 & 75 & 0.002215 & 2.249 & 11.7 & 102.8 & 0.818 & 0.723 \\
\hline 13.7 & 12 & 21.72 & 19.05 & 28.75 & 2033.39 & 76 & 0.002211 & 2.235 & 11.627 & 102.6 & 0.724 & 0.635 \\
\hline 15.38 & 14 & 25.82 & 23.50 & 28.75 & 2033.39 & 76 & 0.002211 & 2.221 & 11.554 & 102.2 & 0.866 & 0.788 \\
\hline 17.47 & 16 & 26.59 & 24.31 & 28.75 & 2033.39 & 77 & 0.002207 & 2.182 & 11.352 & 101.4 & 0.907 & 0.829 \\
\hline
\end{tabular}

Table E. 12: Baseline force data for Run 9

\begin{tabular}{|c|c|c|c|c|c|c|c|c|c|c|c|c|}
\hline Actual AoA & Indicated AoA & Lift & Drag & \multicolumn{2}{|c|}{$P_{-}$atm } & T_tunnel & density & \multicolumn{2}{|c|}{$\Delta \mathrm{P}$} & V & $\mathrm{Cl}$ & $\mathrm{Cd}$ \\
\hline deg & deg & lbs & lbs & $\mathrm{inHg}$ & psf & $\operatorname{deg} F$ & slug. $\mathrm{ft}^{\wedge} 3$ & $\mathrm{inH} 2 \mathrm{O}$ & psf & $\mathrm{ft} / \mathrm{s}$ & - & - \\
\hline-2.39 & -4 & -1.82 & -1.74 & 30.13 & 2130.99 & 62 & 0.002379 & 1.503 & 7.819 & 81.1 & -0.090 & -0.086 \\
\hline-0.14 & -2 & 1.31 & 1.19 & 30.13 & 2130.99 & 65 & 0.002365 & 1.492 & 7.762 & 81.0 & 0.065 & 0.059 \\
\hline 2.49 & 0 & 6.77 & 6.58 & 30.13 & 2130.99 & 68 & 0.002352 & 1.484 & 7.72 & 81.0 & 0.340 & 0.330 \\
\hline 3.73 & 2 & 11.49 & 10.92 & 30.13 & 2130.99 & 70 & 0.002343 & 1.451 & 7.549 & 80.3 & 0.590 & 0.561 \\
\hline 5.27 & 4 & 16.52 & 15.63 & 30.13 & 2130.99 & 70 & 0.002343 & 1.436 & 7.471 & 79.9 & 0.856 & 0.810 \\
\hline 8.16 & 6 & 19.53 & 18.20 & 30.13 & 2130.99 & 70 & 0.002343 & 1.394 & 7.252 & 78.7 & 1.043 & 0.972 \\
\hline 9.7 & 8 & 16.55 & 15.65 & 30.13 & 2130.99 & 70 & 0.002343 & 1.423 & 7.403 & 79.5 & 0.866 & 0.819 \\
\hline 11.65 & 10 & 14.79 & 13.79 & 30.13 & 2130.99 & 70 & 0.002343 & 1.352 & 7.034 & 77.5 & 0.815 & 0.759 \\
\hline 13.7 & 12 & 13.60 & 11.03 & 30.13 & 2130.99 & 68 & 0.002352 & 1.345 & 6.997 & 77.1 & 0.753 & 0.611 \\
\hline 15.38 & 14 & 14.03 & 13.03 & 30.13 & 2130.99 & 66 & 0.002361 & 1.331 & 6.924 & 76.6 & 0.785 & 0.729 \\
\hline 17.47 & 16 & 15.20 & 14.09 & 30.13 & 2130.99 & 62 & 0.002379 & 1.325 & 6.893 & 76.1 & 0.854 & 0.792 \\
\hline
\end{tabular}


Table E. 13: Baseline force data for Run 10

\begin{tabular}{|c|c|c|c|c|c|c|c|c|c|c|c|c|}
\hline Actual AoA & Indicated AoA & Lift & Drag & \multicolumn{2}{|c|}{$\mathrm{P}$ atm } & T_tunnel & density & \multicolumn{2}{|c|}{$\Delta \mathrm{P}$} & $\mathrm{V}$ & $\mathrm{Cl}$ & $\mathrm{Cd}$ \\
\hline deg & deg & Ibs & Ibs & psi & psf & $\operatorname{deg} F$ & slug. $\mathrm{ft}^{\wedge} 3$ & $\mathrm{inH} 2 \mathrm{O}$ & psf & $\mathrm{ft} / \mathrm{s}$ & - & - \\
\hline-2.39 & -4 & -1.69 & 1.62 & 14.158 & 2038.752 & 60 & 0.002285 & 1.51 & 7.856 & 82.9 & -0.083 & 0.080 \\
\hline-0.14 & -2 & 1.42 & -0.26 & 14.158 & 2038.752 & 64 & 0.002267 & 1.512 & 7.866 & 83.3 & 0.070 & -0.013 \\
\hline 2.49 & 0 & 6.92 & -1.90 & 14.158 & 2038.752 & 68 & 0.00225 & 1.493 & 7.767 & 83.1 & 0.345 & -0.095 \\
\hline 3.73 & 2 & 1.73 & -0.69 & 14.158 & 2038.752 & 69 & 0.002246 & 1.495 & 7.778 & 83.2 & 0.086 & -0.034 \\
\hline 5.27 & 4 & 16.59 & -4.39 & 14.158 & 2038.752 & 70 & 0.002242 & 1.435 & 7.465 & 81.6 & 0.861 & -0.228 \\
\hline 8.16 & 6 & 20.55 & -4.96 & 14.158 & 2038.752 & 70 & 0.002242 & 1.398 & 7.273 & 80.6 & 1.095 & -0.264 \\
\hline 9.7 & 8 & 18.09 & -3.80 & 14.158 & 2038.752 & 70 & 0.002242 & 1.36 & 7.075 & 79.4 & 0.991 & -0.208 \\
\hline 11.65 & 10 & 14.49 & -2.49 & 14.158 & 2038.752 & 70 & 0.002242 & 1.36 & 7.075 & 79.4 & 0.793 & -0.136 \\
\hline 13.7 & 12 & 12.40 & -2.95 & 14.158 & 2038.752 & 68 & 0.00225 & 1.365 & 7.101 & 79.4 & 0.676 & -0.161 \\
\hline 15.38 & 14 & 14.39 & -2.81 & 14.158 & 2038.752 & 65 & 0.002263 & 1.352 & 7.034 & 78.8 & 0.792 & -0.155 \\
\hline 17.47 & 16 & 14.93 & -3.06 & 14.158 & 2038.752 & 62 & 0.002276 & 1.341 & 6.976 & 78.3 & 0.829 & -0.170 \\
\hline
\end{tabular}

Table E. 14: Baseline force data for Run 11

\begin{tabular}{|c|c|c|c|c|c|c|c|c|c|c|c|c|}
\hline Actual AoA & Indicated AoA & Lift & Drag & \multicolumn{2}{|c|}{$\mathrm{P}$ _atm } & T_tunnel & density & \multicolumn{2}{|c|}{$\Delta \mathrm{P}$} & V & $\mathrm{Cl}$ & $\mathrm{Cd}$ \\
\hline deg & deg & lbs & lbs & psi & psf & $\operatorname{deg} F$ & slug.ft^3 & inH2O & psf & $\mathrm{ft} / \mathrm{s}$ & - & - \\
\hline-2.39 & -4 & -7.02 & 1.30 & 14.159 & 2038.9 & 68 & 0.00225 & 5.027 & 26.152 & 152.5 & -0.104 & 0.019 \\
\hline-0.14 & -2 & 3.81 & 1.49 & 14.159 & 2038.9 & 77 & 0.002213 & 4.48 & 23.307 & 145.1 & 0.063 & 0.025 \\
\hline 2.49 & 0 & 19.14 & -3.65 & 14.159 & 2038.9 & 82 & 0.002192 & 4.23 & 22.006 & 141.7 & 0.337 & -0.064 \\
\hline 3.73 & 2 & 31.09 & -5.96 & 14.159 & 2038.9 & 86 & 0.002176 & 4.021 & 20.919 & 138.7 & 0.576 & -0.110 \\
\hline 5.27 & 4 & 42.41 & -7.22 & 14.159 & 2038.9 & 89 & 0.002164 & 3.913 & 20.357 & 137.2 & 0.807 & -0.137 \\
\hline 8.16 & 6 & 51.79 & -10.99 & 14.159 & 2038.9 & 91 & 0.002156 & 3.642 & 18.947 & 132.6 & 1.059 & -0.225 \\
\hline 9.7 & 8 & 50.63 & -8.78 & 14.159 & 2038.9 & 73 & 0.002229 & 3.6 & 18.728 & 129.6 & 1.047 & -0.182 \\
\hline 11.65 & 10 & 41.85 & -8.20 & 14.159 & 2038.9 & 80 & 0.0022 & 3.51 & 18.26 & 128.8 & 0.888 & -0.174 \\
\hline 13.7 & 12 & 35.91 & -6.43 & 14.159 & 2038.9 & 84 & 0.002184 & 3.53 & 18.364 & 129.7 & 0.758 & -0.136 \\
\hline 15.38 & 14 & 38.31 & -7.84 & 14.159 & 2038.9 & 87 & 0.002172 & 3.51 & 18.26 & 129.7 & 0.813 & -0.166 \\
\hline 17.47 & 16 & 39.78 & -8.51 & 14.159 & 2038.9 & 90 & 0.00216 & 3.321 & 17.277 & 126.5 & 0.892 & -0.191 \\
\hline
\end{tabular}


Table E. 15: Baseline force data for Run 12

\begin{tabular}{|c|c|c|c|c|c|c|c|c|c|c|c|c|}
\hline Actual AoA & Indicated AoA & Lift & Drag & \multicolumn{2}{|c|}{$P$ atm } & T_tunnel & density & \multicolumn{2}{|c|}{$\Delta \mathrm{P}$} & $\mathrm{V}$ & $\mathrm{Cl}$ & $\mathrm{Cd}$ \\
\hline deg & deg & Ibs & Ibs & psi & psf & $\operatorname{deg} \mathrm{F}$ & slug.ft^3 & inH2O & psf & $\mathrm{ft} / \mathrm{s}$ & - & - \\
\hline-2.39 & -4 & -5.08 & -0.10 & 14.159 & 2038.9 & 94 & 0.002145 & 4.502 & 23.421 & 147.8 & -0.084 & -0.002 \\
\hline-0.14 & -2 & 4.89 & -2.86 & 14.159 & 2038.9 & 92 & 0.002152 & 4.42 & 22.994 & 146.2 & 0.082 & -0.048 \\
\hline 2.49 & 0 & 16.26 & -4.22 & 14.159 & 2038.9 & 95 & 0.002141 & 4.042 & 21.028 & 140.2 & 0.300 & -0.078 \\
\hline 3.73 & 2 & 27.69 & -5.25 & 14.159 & 2038.9 & 97 & 0.002133 & 3.896 & 20.268 & 137.9 & 0.529 & -0.100 \\
\hline 5.27 & 4 & 42.78 & -9.31 & 14.159 & 2038.9 & 96 & 0.002137 & 3.621 & 18.838 & 132.8 & 0.880 & -0.191 \\
\hline 8.16 & 6 & 49.70 & -10.23 & 14.159 & 2038.9 & 98 & 0.002129 & 3.565 & 18.546 & 132.0 & 1.038 & -0.214 \\
\hline 9.7 & 8 & 47.23 & -9.85 & 14.159 & 2038.9 & 93 & 0.002149 & 4.523 & 23.53 & 148.0 & 0.778 & -0.162 \\
\hline 11.65 & 10 & 40.30 & -8.59 & 14.159 & 2038.9 & 94 & 0.002145 & 3.345 & 17.402 & 127.4 & 0.897 & -0.191 \\
\hline 13.7 & 12 & 34.82 & -7.45 & 14.159 & 2038.9 & 98 & 0.002129 & 3.379 & 17.579 & 128.5 & 0.767 & -0.164 \\
\hline 15.38 & 14 & 37.26 & -8.34 & 14.159 & 2038.9 & 97 & 0.002133 & 3.319 & 17.267 & 127.2 & 0.836 & -0.187 \\
\hline 17.47 & 16 & 38.77 & -8.85 & 14.159 & 2038.9 & 96 & 0.002137 & 3.261 & 16.965 & 126.0 & 0.885 & -0.202 \\
\hline
\end{tabular}

Table E. 16: Baseline force data for Run 13

\begin{tabular}{|c|c|c|c|c|c|c|c|c|c|c|c|c|}
\hline Actual AoA & Indicated AoA & Lift & Drag & \multicolumn{2}{|c|}{$P_{\text {atm }}$} & T_tunnel & density & \multicolumn{2}{|c|}{$\Delta \mathrm{P}$} & V & $\mathrm{Cl}$ & $\mathrm{Cd}$ \\
\hline deg & deg & lbs & Ibs & psi & psf & $\operatorname{deg} \mathrm{F}$ & slug.ft^3 & in $\mathrm{H} 2 \mathrm{O}$ & psf & $\mathrm{ft} / \mathrm{s}$ & - & - \\
\hline-2.39 & -4 & -9.49 & -4.99 & 14.148 & 2037.31 & 82 & 0.00219 & 4.681 & 24.352 & 149.1 & -0.151 & -0.079 \\
\hline-0.14 & -2 & -1.67 & -3.69 & 14.148 & 2037.31 & 87 & 0.00217 & 4.732 & 24.618 & 150.6 & -0.026 & -0.058 \\
\hline 2.49 & 0 & 5.34 & -3.23 & 14.148 & 2037.31 & 90 & 0.002159 & 4.051 & 21.075 & 139.7 & 0.098 & -0.059 \\
\hline 3.73 & 2 & 20.13 & -6.83 & 14.148 & 2037.31 & 92 & 0.002151 & 4.282 & 22.276 & 143.9 & 0.350 & -0.119 \\
\hline 5.27 & 4 & 33.49 & -6.51 & 14.148 & 2037.31 & 94 & 0.002143 & 3.864 & 20.102 & 137.0 & 0.645 & -0.125 \\
\hline 8.16 & 6 & 44.78 & -8.89 & 14.148 & 2037.31 & 95 & 0.002139 & 3.685 & 19.171 & 133.9 & 0.905 & -0.180 \\
\hline 9.7 & 8 & 50.86 & -11.29 & 14.148 & 2037.31 & 82 & 0.00219 & 3.751 & 19.514 & 133.5 & 1.010 & -0.224 \\
\hline 11.65 & 10 & 46.68 & -10.93 & 14.148 & 2037.31 & 89 & 0.002163 & 3.522 & 18.323 & 130.2 & 0.987 & -0.231 \\
\hline 13.7 & 12 & 39.26 & -9.83 & 14.148 & 2037.31 & 90 & 0.002159 & 3.435 & 17.87 & 128.7 & 0.851 & -0.213 \\
\hline 15.38 & 14 & 34.86 & -7.68 & 14.148 & 2037.31 & 92 & 0.002151 & 3.362 & 17.49 & 127.5 & 0.772 & -0.170 \\
\hline 17.47 & 16 & 38.50 & -9.12 & 14.148 & 2037.31 & 94 & 0.002143 & 3.321 & 17.277 & 127.0 & 0.863 & -0.204 \\
\hline
\end{tabular}




\section{Appendix}

F

Active Circulation Control Model Calibration Curves 


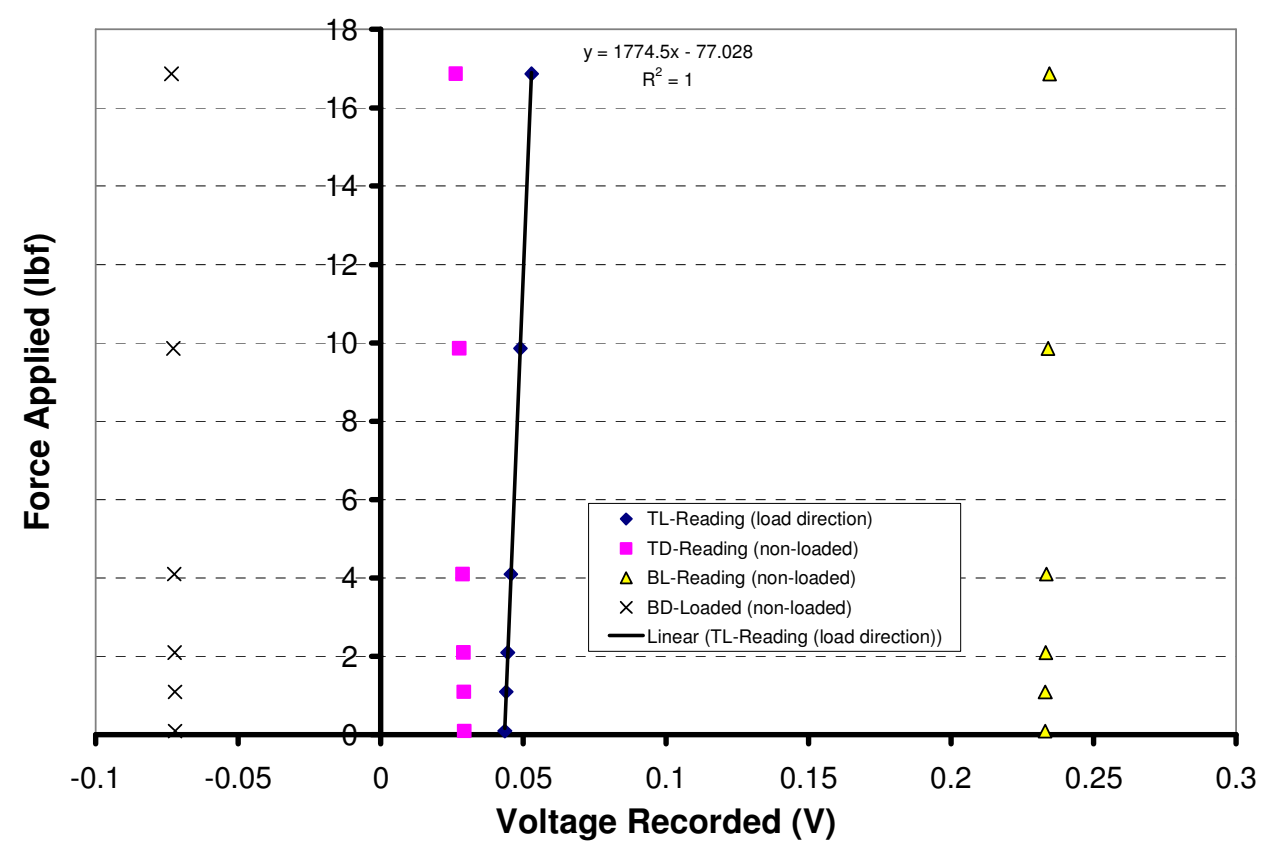

Figure F. 1: Top lift calibration with the active circulation control model

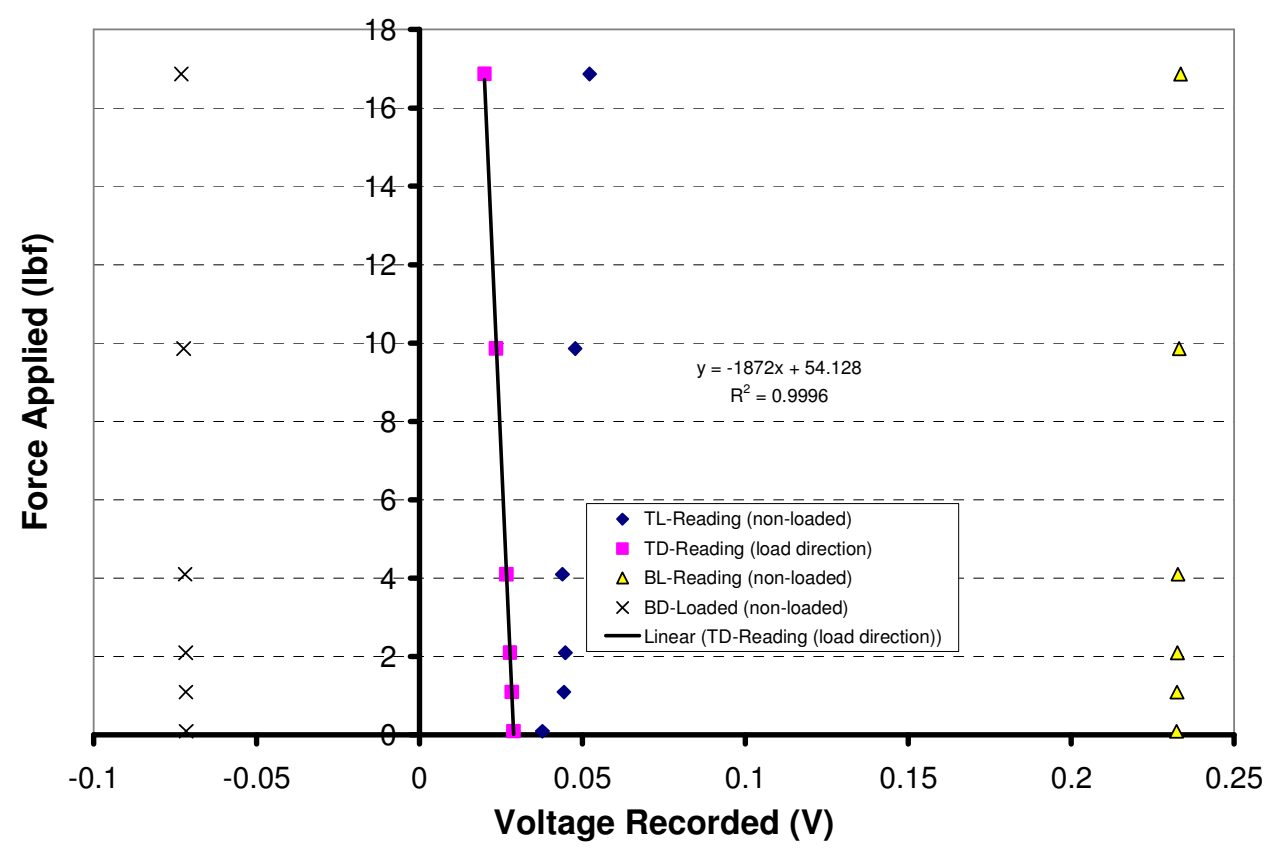

Figure F. 2: Top drag calibration with the active circulation control model 


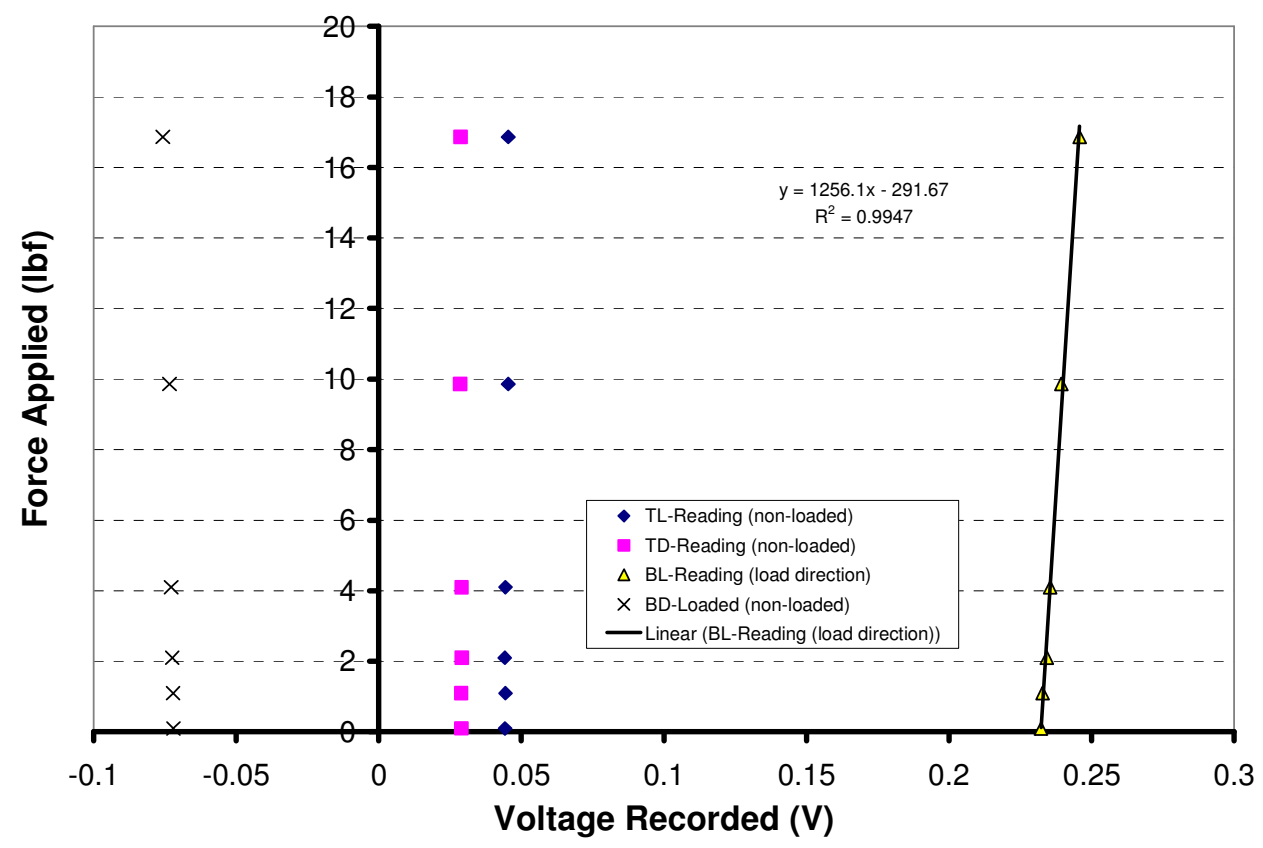

Figure F. 3: Bottom lift calibration with the active circulation control model

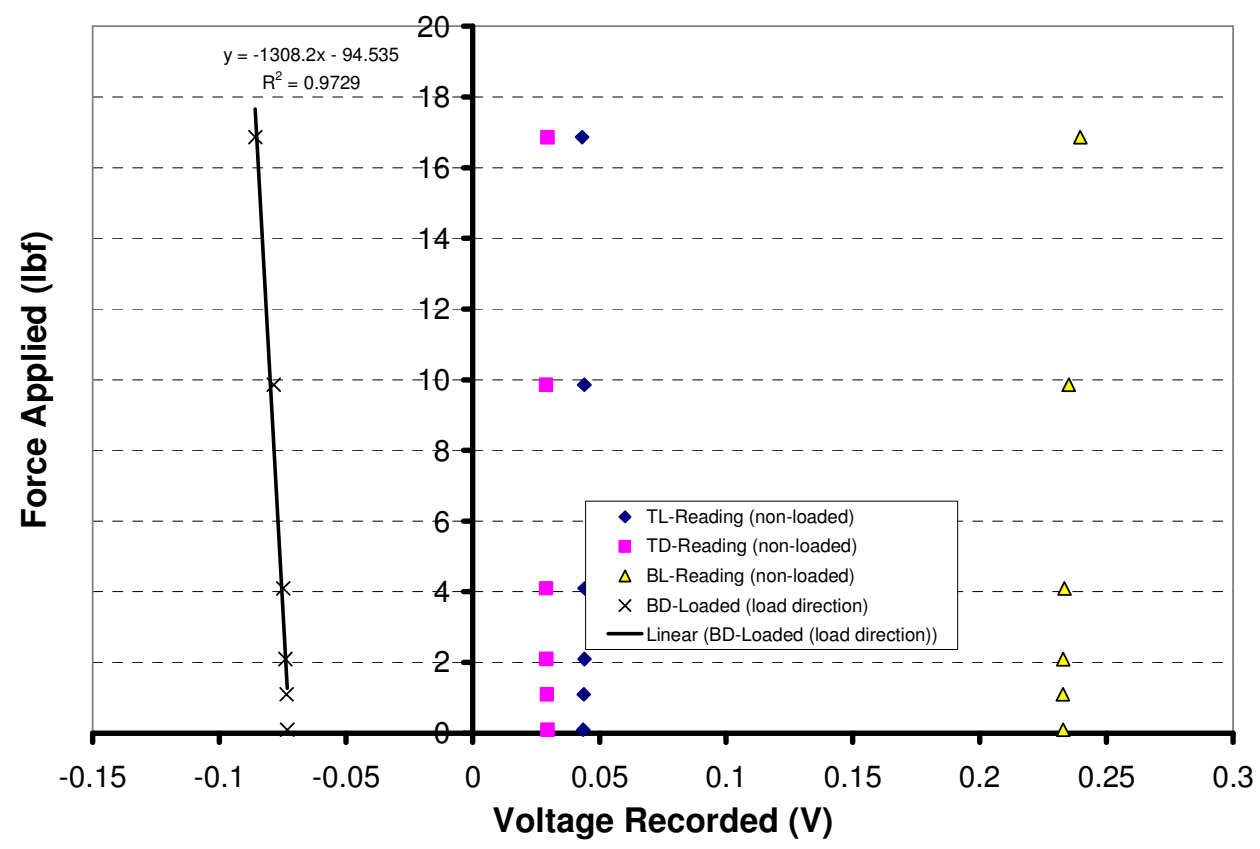

Figure F. 4: Bottom drag calibration with the active circulation control model 


\section{Appendix}

G

Summary of the Active Circulation Control Model Experimental Data 
Table G. 1: Summary of lift and drag coefficients found at 0.68 degrees angle of attack for the trailing edge blowing slot

\begin{tabular}{|c|c|c|}
\hline TE Cm & $\mathrm{Cl}$ & $\mathrm{Cd}$ \\
\hline- & - & - \\
\hline 0.0000 & 0.0889 & 0.0701 \\
\hline 0.0000 & 0.0775 & 0.0675 \\
\hline 0.0000 & 0.1150 & 0.0774 \\
\hline 0.0031 & 0.0907 & 0.0612 \\
\hline 0.0061 & 0.1277 & 0.0525 \\
\hline 0.0063 & 0.1082 & 0.0635 \\
\hline 0.0063 & 0.0897 & 0.0567 \\
\hline 0.0099 & 0.1045 & 0.0612 \\
\hline 0.0100 & 0.0902 & 0.0527 \\
\hline 0.0101 & 0.1742 & 0.0279 \\
\hline 0.0121 & 0.1179 & 0.0637 \\
\hline 0.0127 & 0.1269 & 0.0380 \\
\hline 0.0190 & 0.1073 & 0.0567 \\
\hline 0.0201 & 0.1395 & 0.0309 \\
\hline 0.0207 & 0.2024 & 0.0398 \\
\hline 0.0209 & 0.1756 & 0.0087 \\
\hline 0.0327 & 0.1734 & -0.0084 \\
\hline 0.0327 & 0.1866 & 0.0332 \\
\hline
\end{tabular}

Table G. 2: Summary of the lift and drag coefficients at 0.68 degrees angle of attack for the leading edge blowing slot

\begin{tabular}{|c|c|c|}
\hline LE Cm & $\mathrm{Cl}$ & $\mathrm{Cd}$ \\
\hline- & - & - \\
\hline 0.0000 & 0.0889 & 0.0701 \\
\hline 0.0000 & 0.0775 & 0.0675 \\
\hline 0.0000 & 0.1150 & 0.0774 \\
\hline 0.0030 & 0.1004 & 0.0592 \\
\hline 0.0061 & 0.1376 & 0.0522 \\
\hline 0.0101 & 0.1862 & 0.0348 \\
\hline
\end{tabular}


Table G. 3: Summary of the lift and drag coefficients at 0.68 degrees angle of attack and leading edge regulated pressure at $10 \mathrm{psig}$

\begin{tabular}{|c|c|c|c|}
\hline TE Cm & LE Cm & Cl & Cd \\
\hline- & - & - & - \\
\hline 0.0000 & 0.0030 & 0.1004 & 0.0592 \\
\hline 0.0030 & 0.0030 & 0.0991 & 0.0593 \\
\hline 0.0062 & 0.0030 & 0.1042 & 0.0570 \\
\hline 0.0099 & 0.0030 & 0.1039 & 0.0522 \\
\hline 0.0190 & 0.0058 & 0.0989 & 0.0384 \\
\hline 0.0121 & 0.0058 & 0.1174 & 0.0514 \\
\hline 0.0061 & 0.0061 & 0.1368 & 0.0389 \\
\hline 0.0000 & 0.0061 & 0.1376 & 0.0522 \\
\hline 0.0327 & 0.0100 & 0.1700 & -0.0005 \\
\hline 0.0208 & 0.0101 & 0.1984 & 0.0127 \\
\hline 0.0000 & 0.0101 & 0.1862 & 0.0348 \\
\hline 0.0101 & 0.0101 & 0.1887 & 0.0190 \\
\hline
\end{tabular}

Table G. 4: Summary of the lift and drag coefficients at 0.68 degrees angle of attack and leading edge regulated pressure at $20 \mathrm{psig}$

\begin{tabular}{|c|c|c|c|}
\hline TE Cm & LE Cm & $\mathrm{Cl}$ & $\mathrm{Cd}$ \\
\hline- & - & - & - \\
\hline 0.0030 & 0.0062 & 0.0930 & 0.0560 \\
\hline 0.0062 & 0.0062 & 0.0995 & 0.0503 \\
\hline 0.0099 & 0.0063 & 0.0931 & 0.0457 \\
\hline 0.0190 & 0.0120 & 0.0899 & 0.0253 \\
\hline 0.0121 & 0.0121 & 0.1130 & 0.0378 \\
\hline 0.0061 & 0.0126 & 0.1359 & 0.0314 \\
\hline 0.0327 & 0.0207 & 0.1546 & -0.0196 \\
\hline 0.0101 & 0.0208 & 0.1830 & 0.0031 \\
\hline 0.0208 & 0.0208 & 0.1464 & -0.0129 \\
\hline
\end{tabular}

Table G. 5: Summary of the lift and drag coefficients at 0.68 degrees angle of attack and leading edge regulated pressure at $30 \mathrm{psig}$

\begin{tabular}{|c|c|c|c|}
\hline TE Cm & LE Cm & Cl & Cd \\
\hline- & - & - & - \\
\hline 0.0030 & 0.0099 & 0.0896 & 0.0500 \\
\hline 0.0062 & 0.0099 & 0.0910 & 0.0467 \\
\hline 0.0099 & 0.0099 & 0.0856 & 0.0384 \\
\hline 0.0193 & 0.0193 & 0.0756 & 0.0107 \\
\hline 0.0061 & 0.0201 & 0.1395 & 0.0201 \\
\hline 0.0127 & 0.0201 & 0.1027 & 0.0155 \\
\hline 0.0327 & 0.0327 & 0.1369 & -0.0443 \\
\hline 0.0101 & 0.0330 & 0.1696 & -0.0162 \\
\hline 0.0208 & 0.0330 & 0.1551 & -0.0357 \\
\hline
\end{tabular}




\section{Appendix}

H

\section{Additional Computational Grid Images}




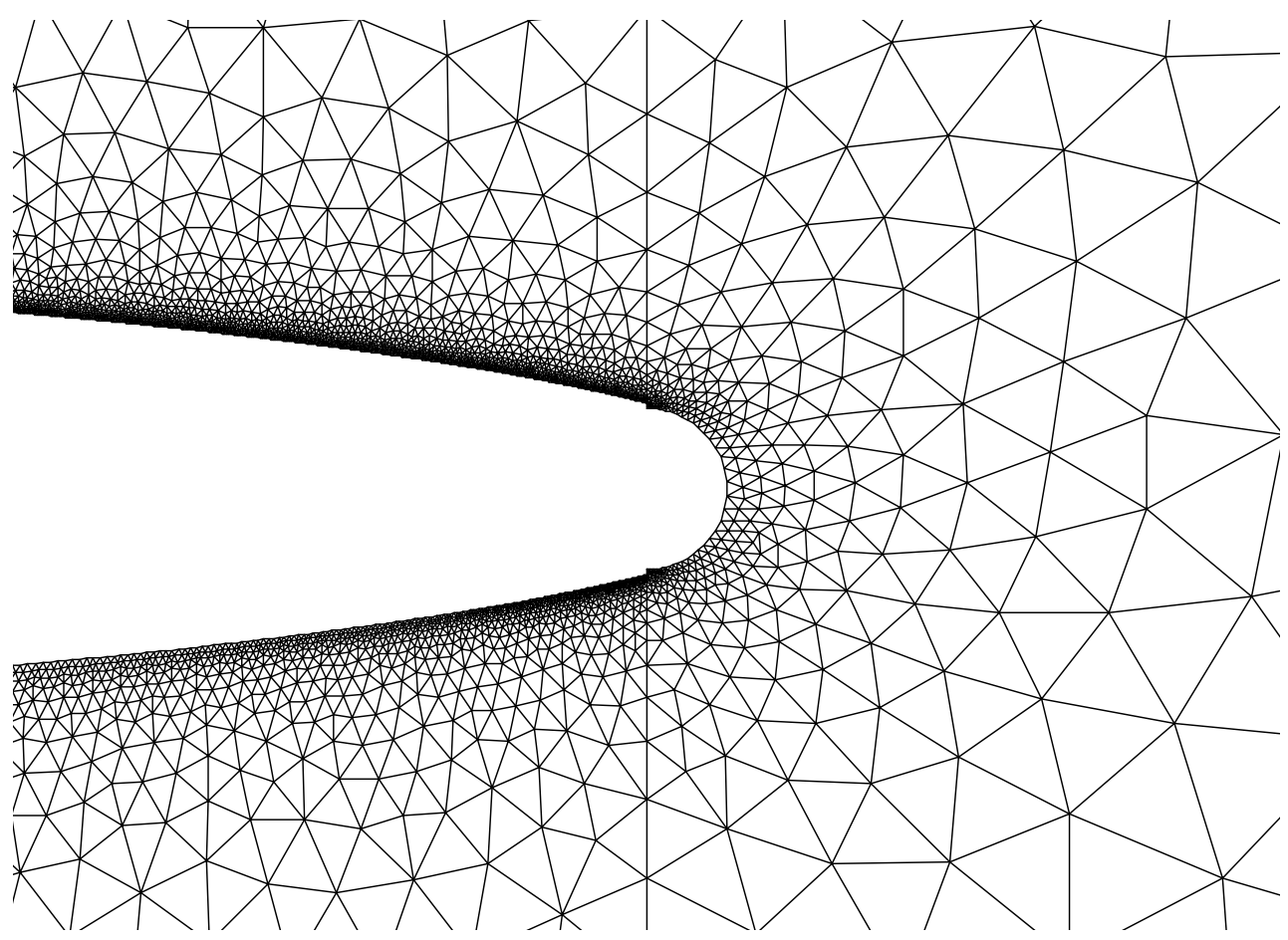

Figure H. 1: Locally enlarged view of the 2-D grid near the trailing edge of the model

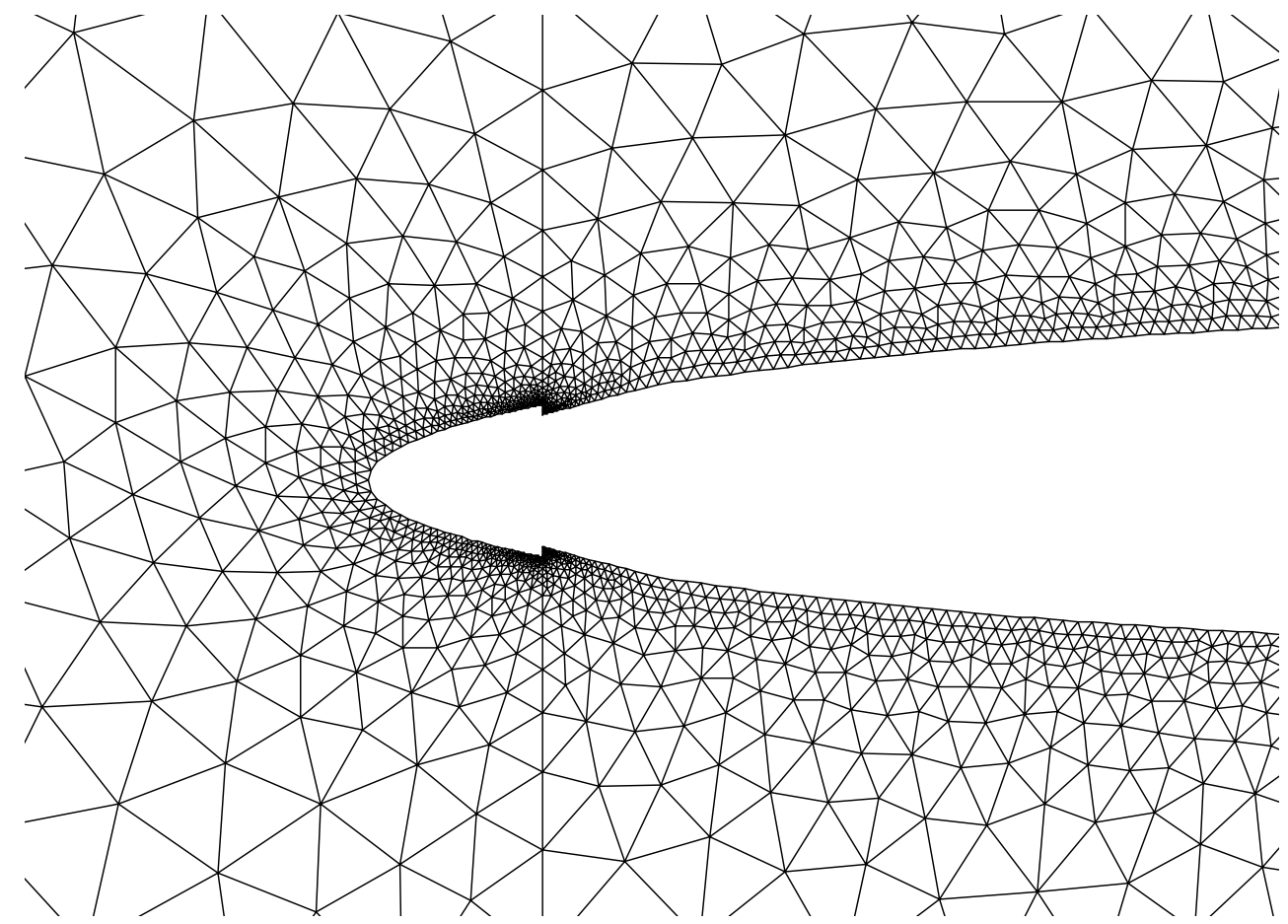

Figure H. 2: Locally enlarged view of the 2-D grid near the leading edge 
Appendix

I

Summary of CFD Data, Tables and Figures 
Table I. 1: Summary of the computational data with various wind speeds and no circulation control blowing

\begin{tabular}{|c|c|c|c|c|c|c|}
\hline $\begin{array}{l}\text { Freestream } \\
\text { Velocity }\end{array}$ & $\begin{array}{c}\text { Velocity Ratio } \\
\mathrm{V}_{\text {jet }} / \mathrm{V}_{\text {f.s. }}\end{array}$ & Lift Force & Drag Force & $\begin{array}{c}\text { Lift } \\
\text { Coefficient }\end{array}$ & $\begin{array}{c}\text { Drag } \\
\text { Coefficient }\end{array}$ & $\mathrm{L} / \mathrm{D}$ \\
\hline $\mathrm{ft} / \mathrm{s}$ & - & lbf & Ibf & - & - & - \\
\hline 82.021 & $\mathrm{~N} / \mathrm{A}$ & 1.844 & 0.45 & 0.089 & 0.022 & 4.05 \\
\hline 150 & $\mathrm{~N} / \mathrm{A}$ & -1.624 & 7.66 & -0.024 & 0.111 & -0.22 \\
\hline 450 & $\mathrm{~N} / \mathrm{A}$ & -17.37 & 54.19 & -0.028 & 0.087 & -0.32 \\
\hline 600 & $\mathrm{~N} / \mathrm{A}$ & -35.94 & 89.14 & -0.033 & 0.081 & -0.41 \\
\hline 750 & $\mathrm{~N} / \mathrm{A}$ & -58.98 & 130.57 & -0.034 & 0.076 & -0.45 \\
\hline 900 & $\mathrm{~N} / \mathrm{A}$ & -83.18 & 177.93 & -0.033 & 0.072 & -0.46 \\
\hline 1050 & $\mathrm{~N} / \mathrm{A}$ & -113.78 & 230.73 & -0.034 & 0.068 & -0.5 \\
\hline 1200 & $\mathrm{~N} / \mathrm{A}$ & -149.63 & 288.80 & -0.034 & 0.065 & -0.52 \\
\hline 1350 & $\mathrm{~N} / \mathrm{A}$ & -191.35 & 351.85 & -0.034 & 0.063 & -0.54 \\
\hline 1500 & $\mathrm{~N} / \mathrm{A}$ & -239.32 & 419.44 & -0.035 & 0.061 & -0.57 \\
\hline
\end{tabular}

Table I. 2: Summary of the computational data with various wind speeds and an upper surface trailing edge blowing of $1400 \mathrm{ft} / \mathrm{s}$

\begin{tabular}{|c|c|c|c|c|c|c|c|c|}
\hline $\begin{array}{c}\text { Freestream } \\
\text { Velocity }\end{array}$ & $\begin{array}{c}\text { Blowing } \\
\text { Coefficient }\end{array}$ & $\begin{array}{c}\text { Velocity Ratio } \\
\mathrm{V}_{\text {jet }} / \mathrm{V}_{\text {f.s. }}\end{array}$ & $\begin{array}{l}\text { Lift } \\
\text { Force }\end{array}$ & $\begin{array}{l}\text { Drag } \\
\text { Force }\end{array}$ & $\begin{array}{c}\text { Lift } \\
\text { Coefficient }\end{array}$ & $\begin{array}{c}\text { Drag } \\
\text { Coefficient }\end{array}$ & $\mathrm{L} / \mathrm{D}$ & $\begin{array}{c}\text { Lift } \\
\text { Augmentation }\end{array}$ \\
\hline $\mathrm{ft} / \mathrm{s}$ & - & - & Ibf & lbf & - & - & - & - \\
\hline 82.021 & 0.4938 & 17.07 & 89.19 & 40.82 & 4.316 & 1.975 & 2.19 & 48.494 \\
\hline 150 & 0.1476 & 9.33 & 189.41 & 52.43 & 2.741 & 0.759 & 3.61 & 114.208 \\
\hline 450 & 0.0164 & 3.11 & 712.17 & 106.20 & 1.145 & 0.171 & 6.70 & 40.893 \\
\hline 600 & 0.0092 & 2.33 & 896.92 & 133.73 & 0.811 & 0.121 & 6.70 & 24.576 \\
\hline 750 & 0.0059 & 1.87 & 1016.48 & 167.17 & 0.588 & 0.097 & 6.06 & 17.294 \\
\hline 900 & 0.0041 & 1.56 & 1111.22 & 208.48 & 0.447 & 0.084 & 5.32 & 13.545 \\
\hline 1050 & 0.003 & 1.33 & 1154.32 & 255.39 & 0.341 & 0.075 & 4.55 & 10.029 \\
\hline 1200 & 0.0023 & 1.17 & 1168.33 & 308.66 & 0.264 & 0.070 & 3.77 & 7.765 \\
\hline 1350 & 0.0018 & 1.04 & 1106.84 & 366.32 & 0.198 & 0.065 & 3.05 & 5.824 \\
\hline 1500 & 0.0015 & 0.93 & 946.52 & 428.13 & 0.137 & 0.062 & 2.21 & 3.914 \\
\hline
\end{tabular}


Table I. 3: Summary of the computational data with various wind speeds, upper surface leading edge blowing of $600 \mathrm{ft} / \mathrm{s}$ and trailing edge jet velocity of $1400 \mathrm{ft} / \mathrm{s}$

\begin{tabular}{|c|c|c|c|c|c|c|c|c|}
\hline $\begin{array}{c}\text { Freestream } \\
\text { Velocity }\end{array}$ & $\begin{array}{l}\text { LE Blowing } \\
\text { Coefficient }\end{array}$ & $\begin{array}{l}\text { TE Blowing } \\
\text { Coefficient }\end{array}$ & $\begin{array}{l}\text { Lift } \\
\text { Force }\end{array}$ & $\begin{array}{l}\text { Drag } \\
\text { Force }\end{array}$ & $\begin{array}{c}\text { Lift } \\
\text { Coefficient }\end{array}$ & $\begin{array}{c}\text { Drag } \\
\text { Coefficient }\end{array}$ & $L / D$ & $\begin{array}{c}\text { Lift } \\
\text { Augmentation }\end{array}$ \\
\hline $\mathrm{ft} / \mathrm{s}$ & - & - & lbf & Ibf & - & - & - & - \\
\hline 82.021 & 0.0907 & 0.494 & 97.17 & 45.02 & 4.702 & 2.179 & 2.16 & 52.831 \\
\hline 150 & 0.0271 & 0.148 & 197.29 & 55.61 & 2.855 & 0.805 & 3.55 & 118.958 \\
\hline 450 & 0.003 & 0.016 & 714.50 & 107.57 & 1.149 & 0.173 & 6.64 & 41.036 \\
\hline 600 & 0.0017 & 0.009 & 897.45 & 134.70 & 0.812 & 0.122 & 6.66 & 24.606 \\
\hline 750 & 0.0011 & 0.006 & 1015.77 & 167.84 & 0.588 & 0.097 & 6.06 & 17.294 \\
\hline 900 & 0.0008 & 0.004 & 1109.08 & 209.21 & 0.446 & 0.084 & 5.31 & 13.515 \\
\hline 1050 & 0.0006 & 0.003 & 1151.43 & 255.89 & 0.340 & 0.076 & 4.47 & 10.000 \\
\hline 1200 & 0.0004 & 0.002 & 1164.51 & 308.88 & 0.263 & 0.070 & 3.76 & 7.735 \\
\hline 1350 & 0.0003 & 0.002 & 1103.60 & 366.30 & 0.197 & 0.065 & 3.03 & 5.794 \\
\hline 1500 & 0.0003 & 0.001 & 941.94 & 427.72 & 0.136 & 0.062 & 2.19 & 3.886 \\
\hline
\end{tabular}


The remaining figures utilize the acronym of TEU to represent the trailing edge upper surface jet followed by the velocity of the jet in feet per second. The acronym TEL represents the lower surface trailing edge jet, LEU and LEL represent the leading edge, upper and lower jets, respectively. Combined computational cases incorporate multiple acronyms, for example LEU600TEU1400 represents the leading edge jet at $600 \mathrm{ft} / \mathrm{s}$ and trailing edge blowing at $1400 \mathrm{ft} / \mathrm{s}$. Unless specified the freestream velocity was $82 \mathrm{ft} / \mathrm{s}$, adding a $\mathrm{V}$ and a number before the blowing condition acronyms indicate the updated velocity.

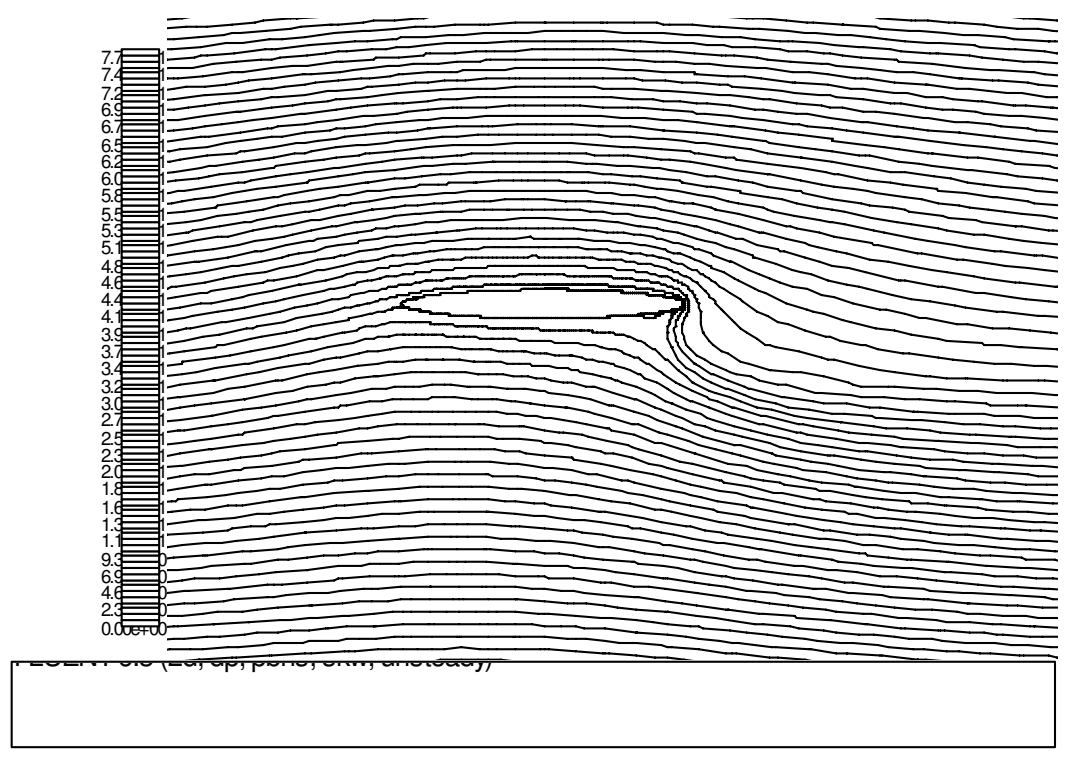

Figure I. 1: Streamlines for trailing edge upper (TEU) blowing at $1200 \mathrm{ft} / \mathrm{s}$ 


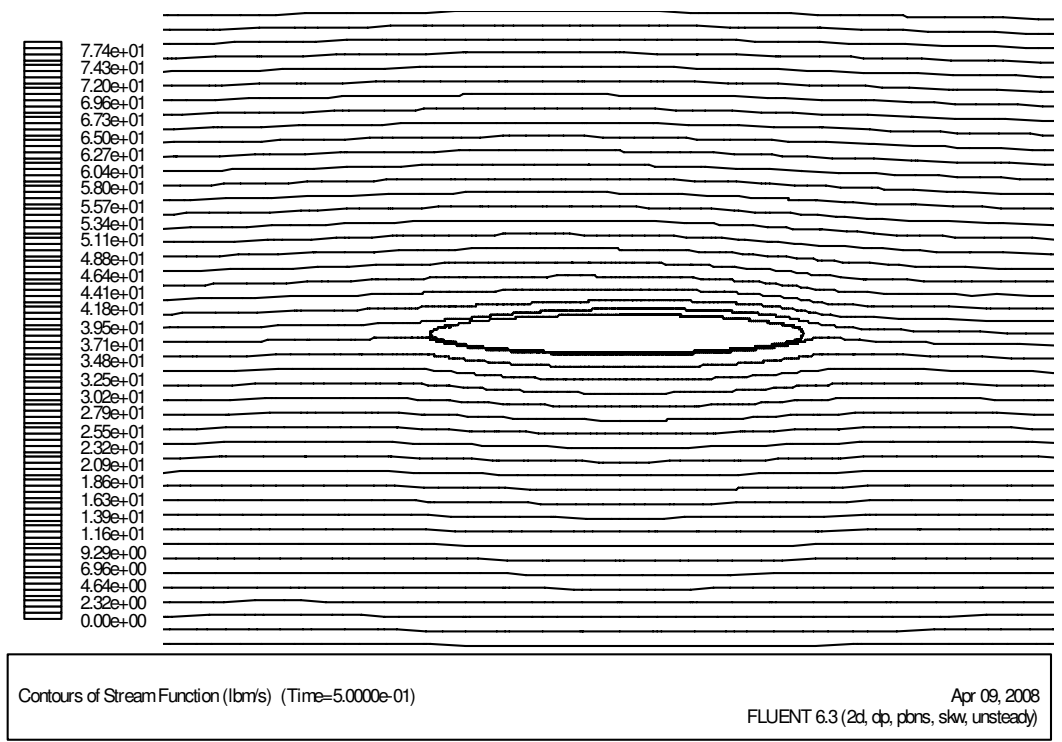

Figure I. 2: Streamlines for the leading edge upper (LEU) blowing at $800 \mathrm{ft} / \mathrm{s}$ case

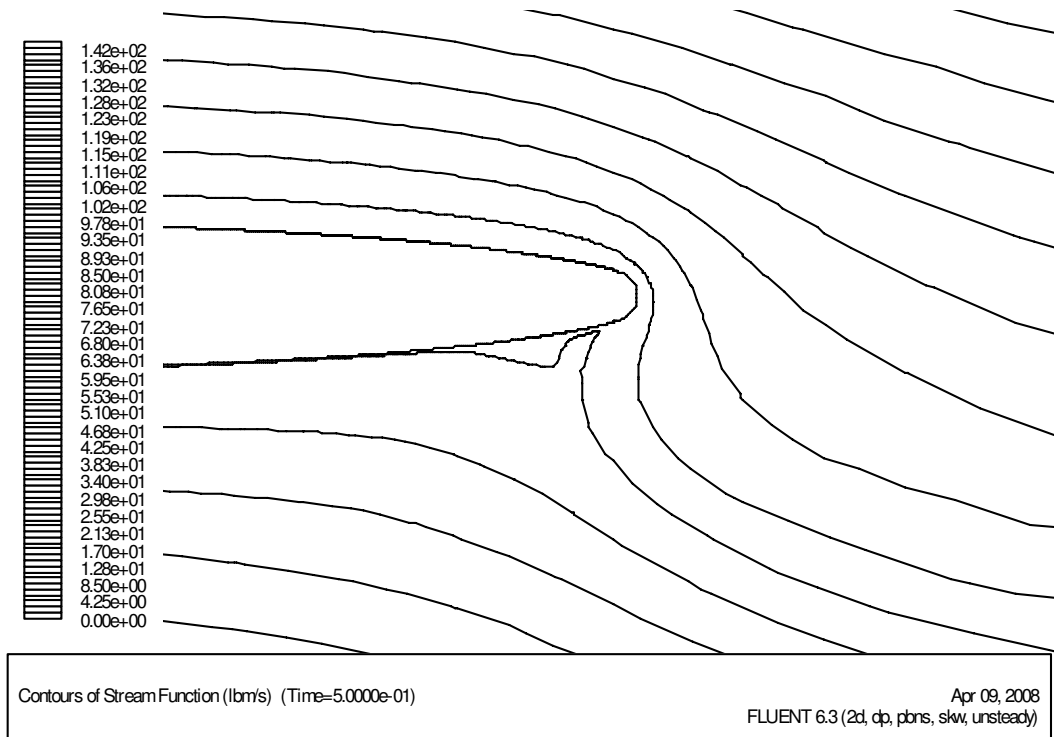

Figure I. 3: Trailing edge streamlines for velocity 150 and LEU600TEU1400 


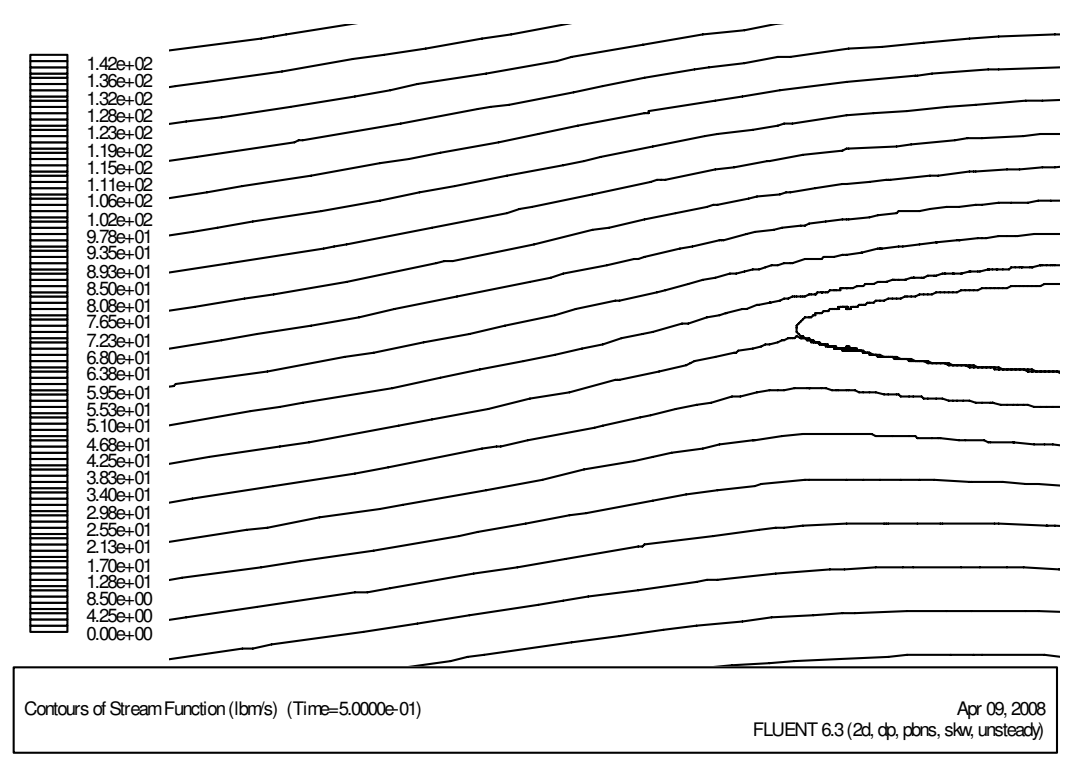

Figure I. 4: Leading edge streamlines for V150LEU600TEU1400

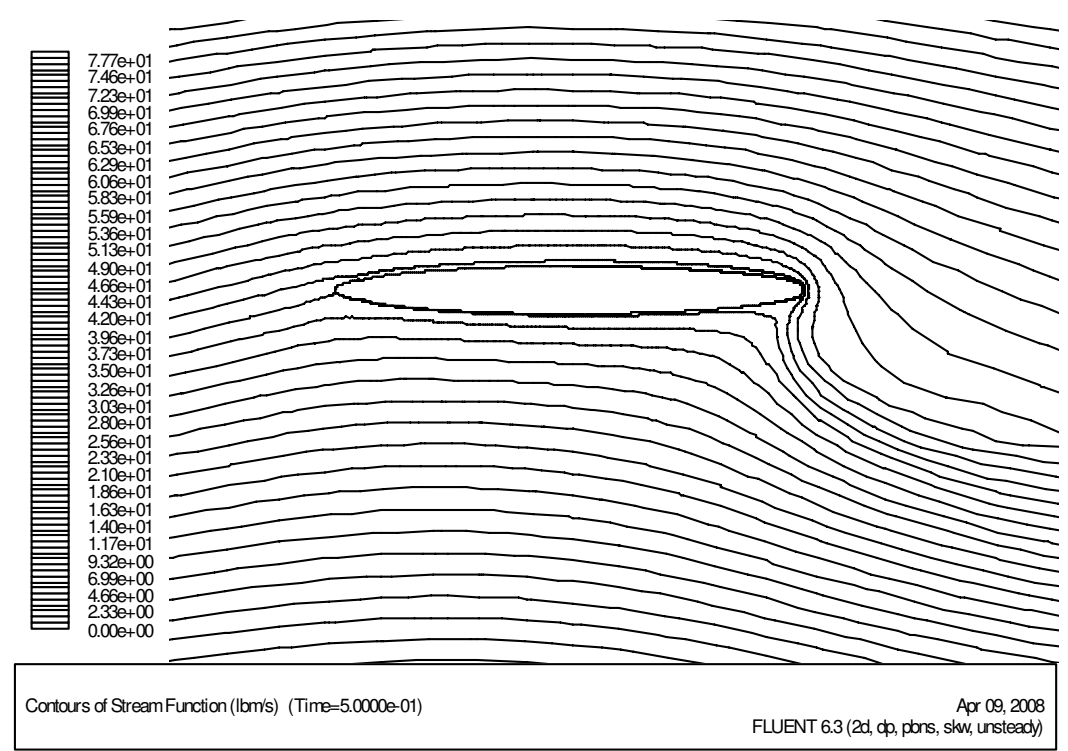

Figure I. 5: Streamlines for LEL600TEU1400 


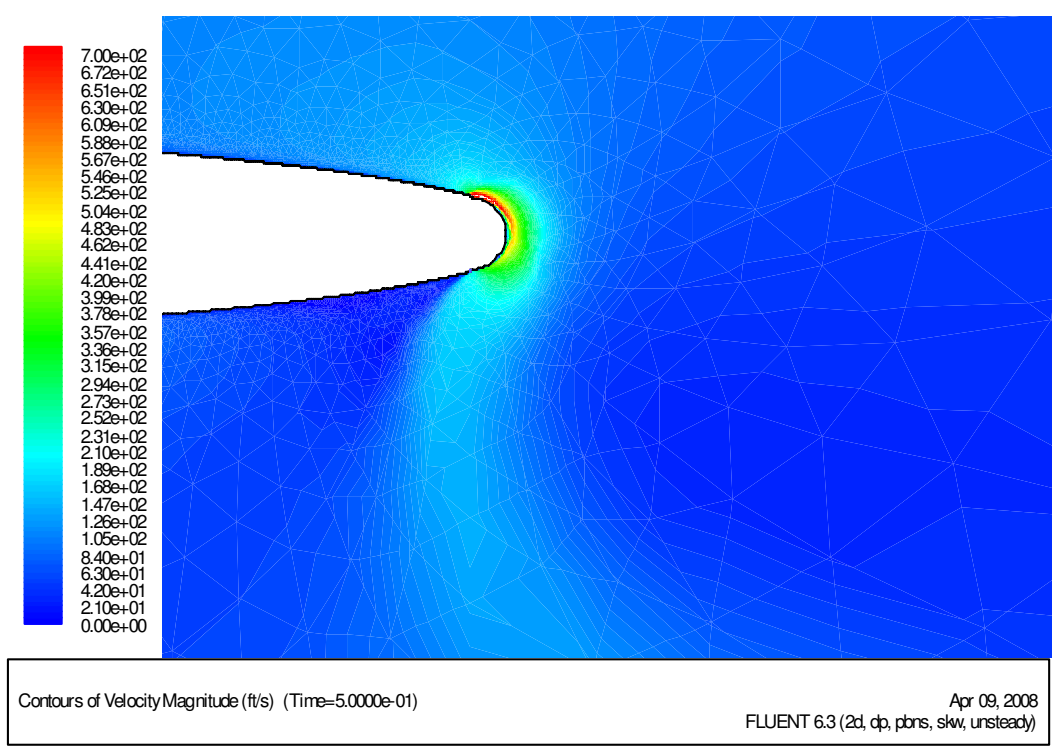

Figure I. 6: Velocity magnitude contour near trailing edge for LEL600TEU1400

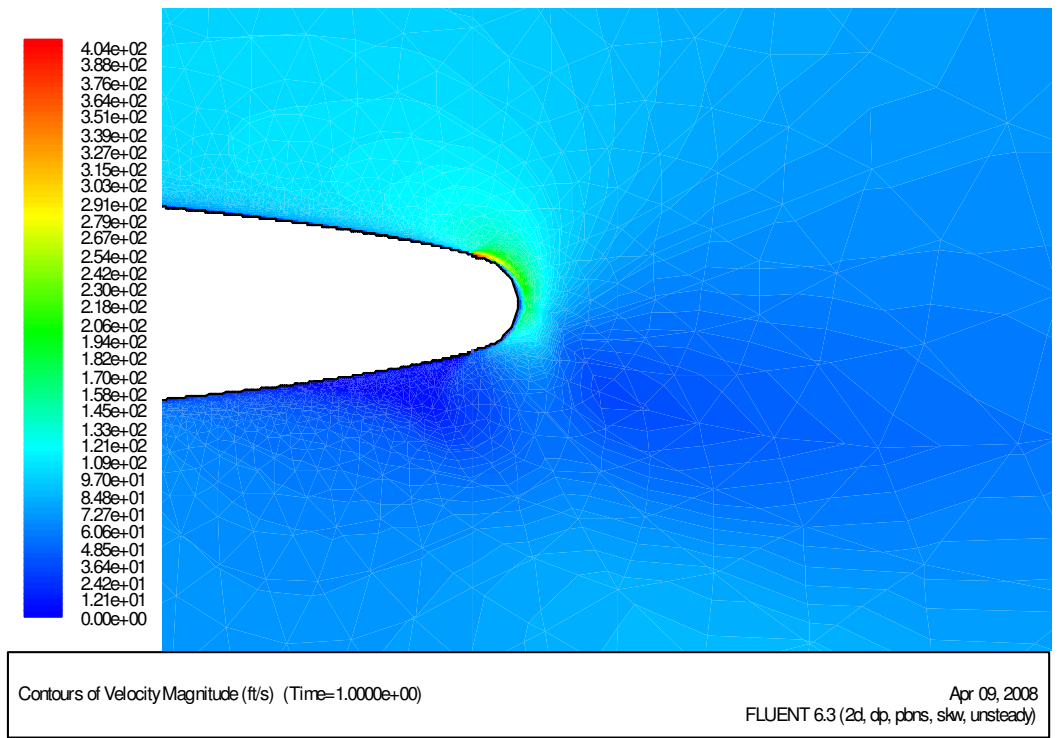

Figure I. 7: Trailing edge velocity magnitude contour for TEU400 


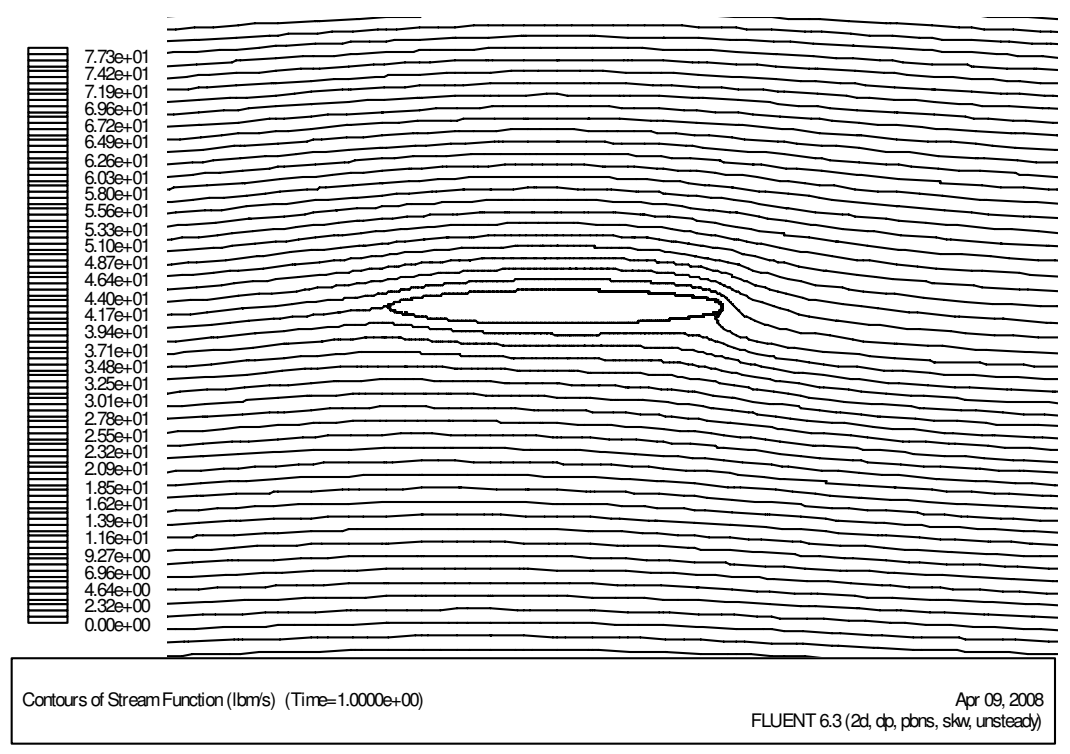

Figure I. 8: Streamlines for TEU400

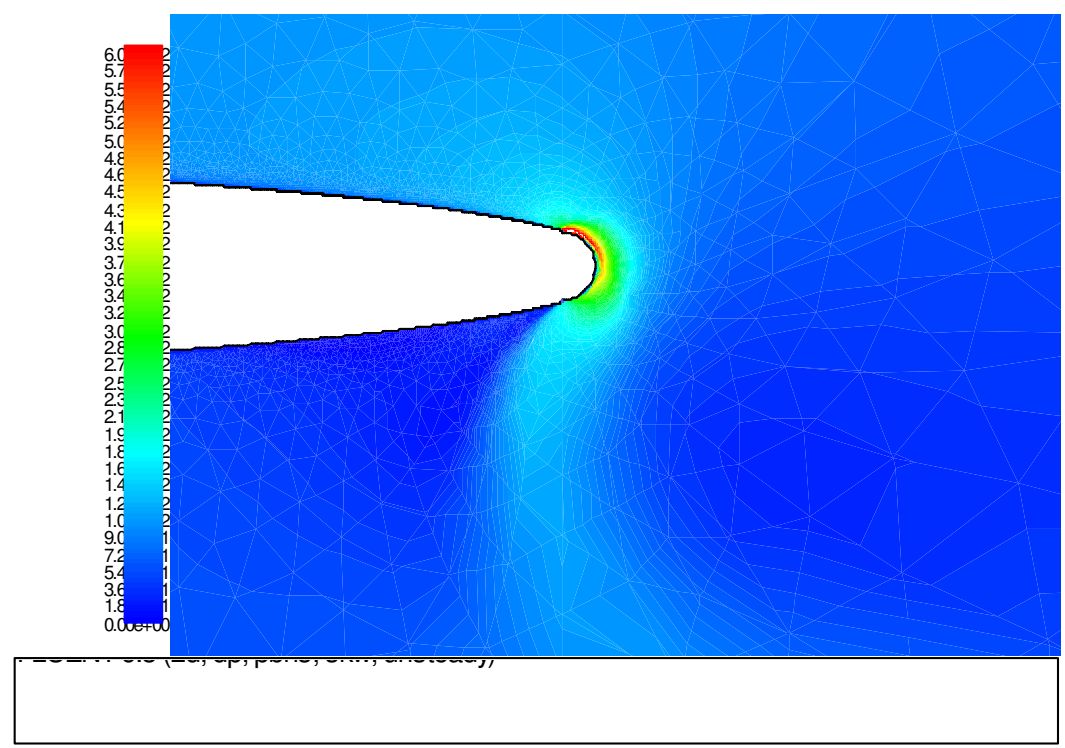

Figure I. 9: Trailing edge velocity magnitude contour for TEU1200 


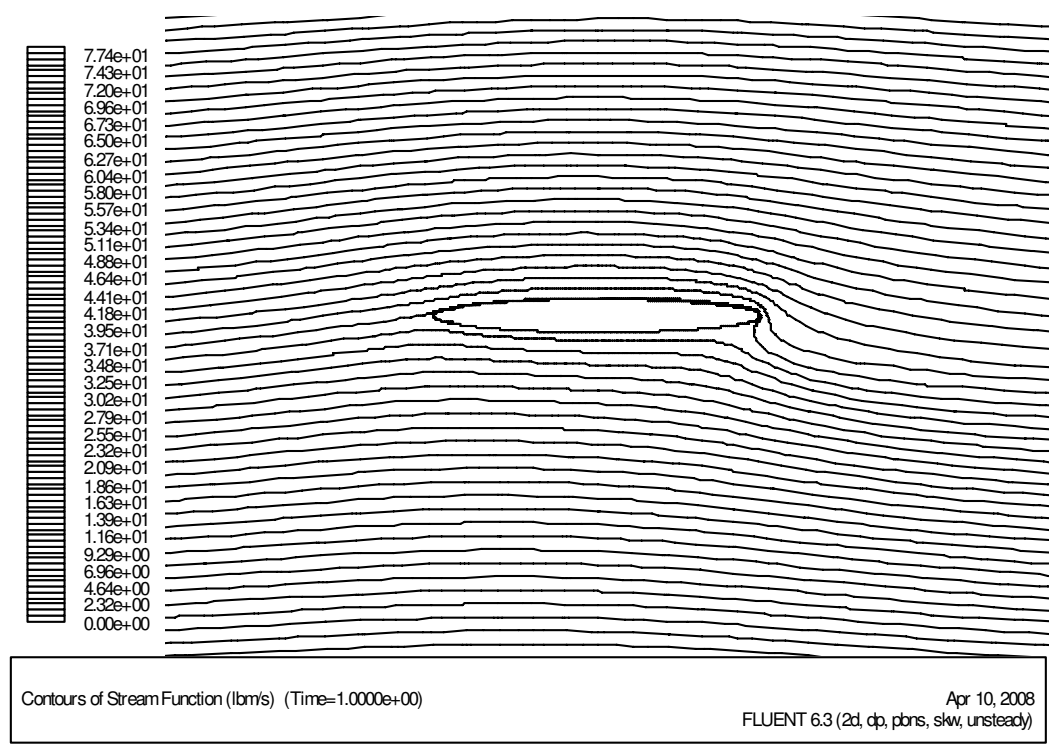

Figure I. 10: Streamlines for LEU200TEU600

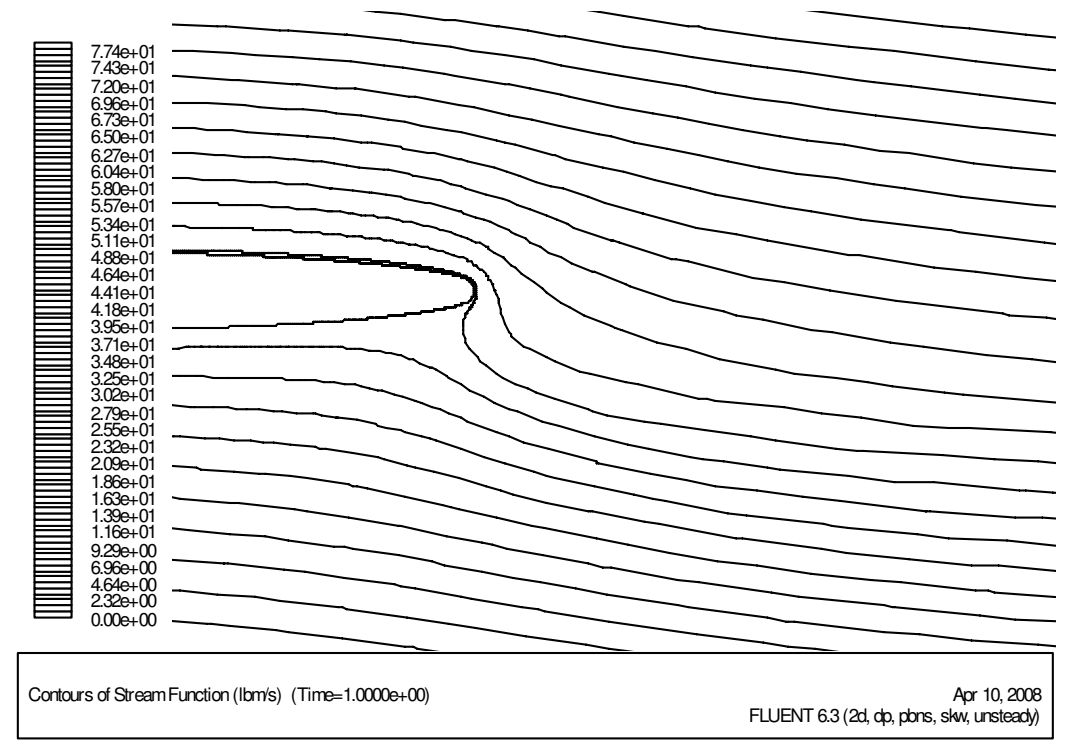

Figure I. 11: Trailing edge streamlines for LEU200TEU600 


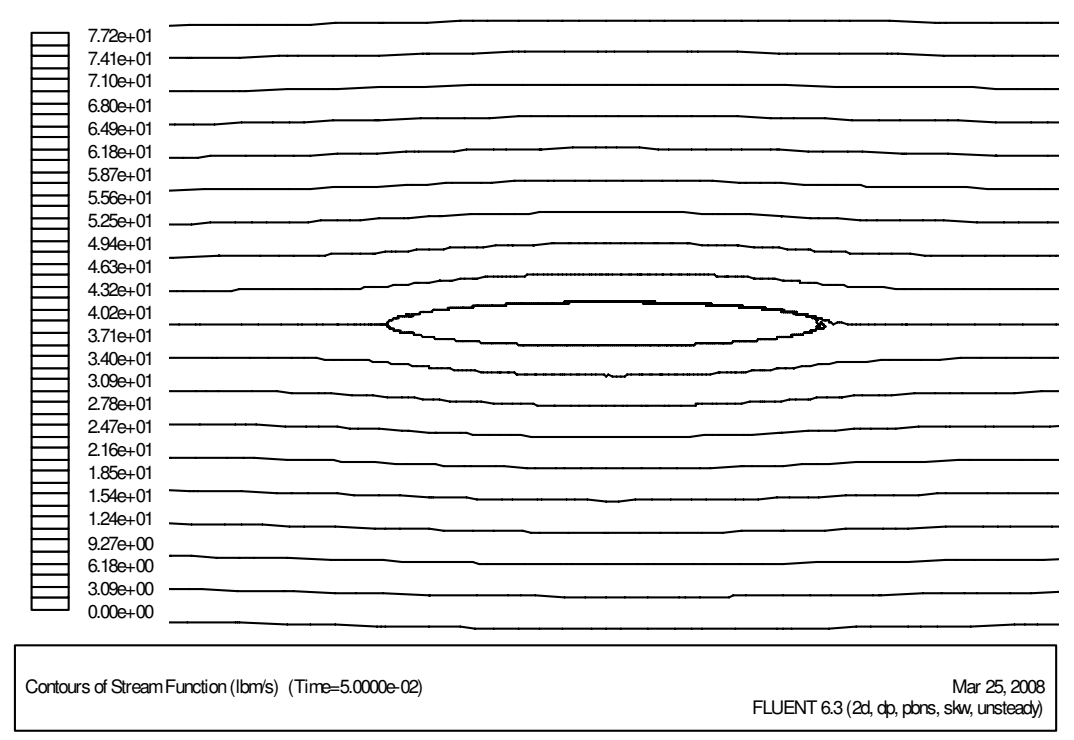

Figure I. 12: Streamlines for LEU200

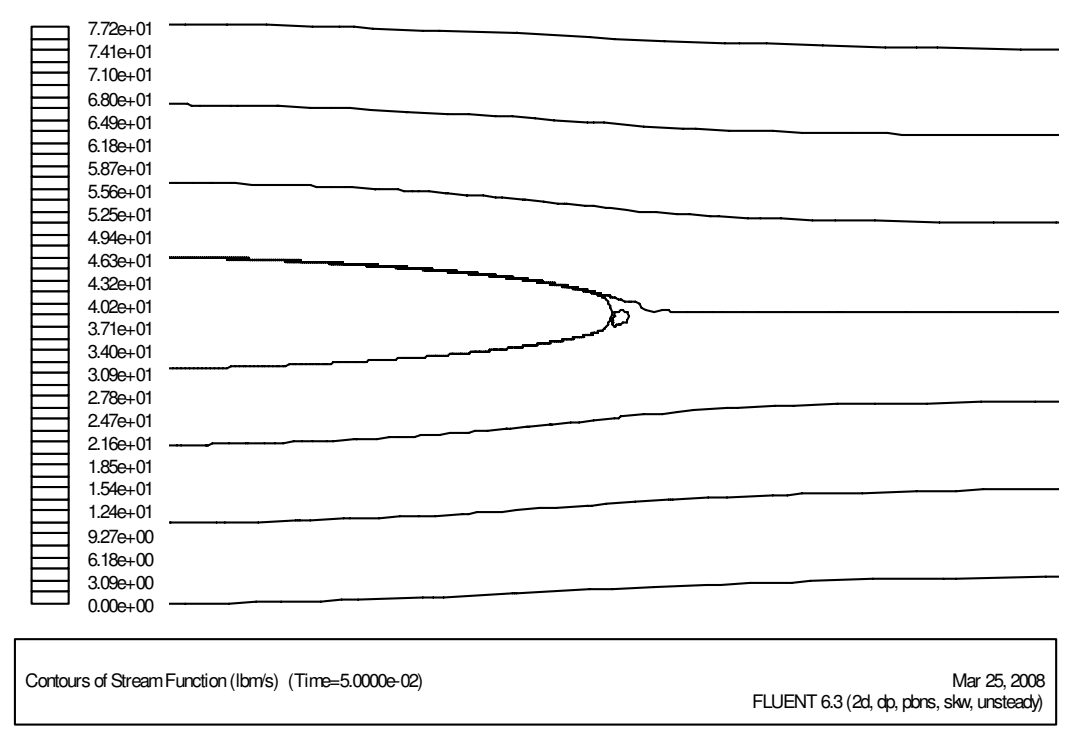

Figure I. 13: Trailing edge streamlines for LEU200 


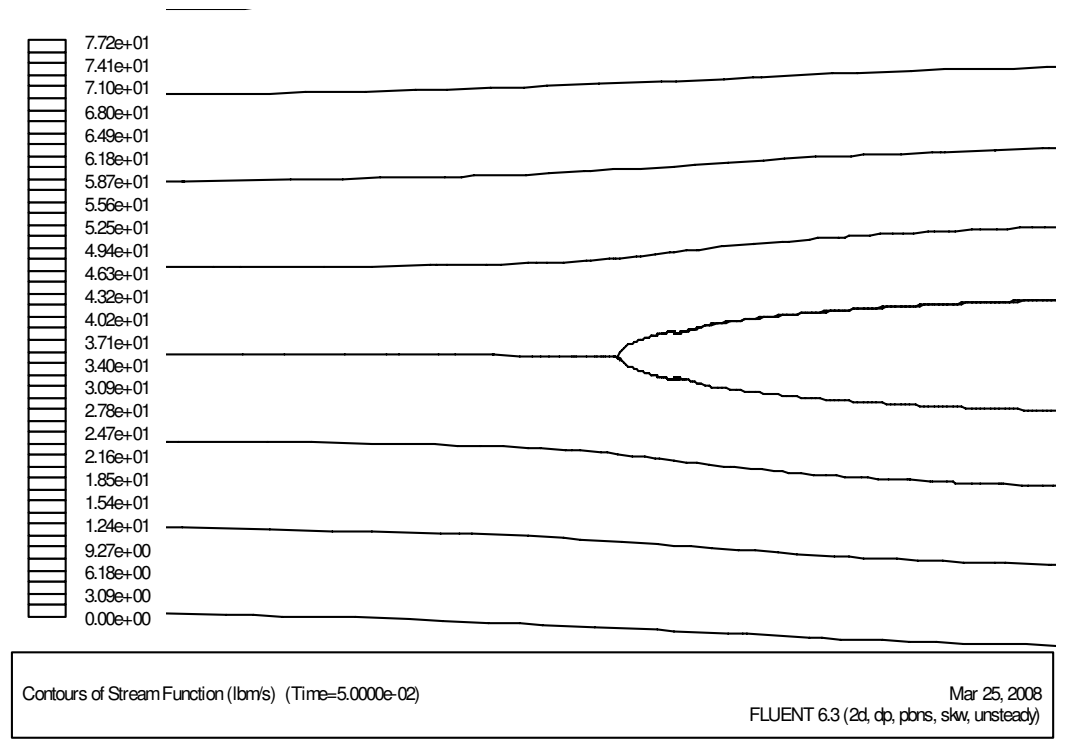

Figure I. 14: Leading edge streamlines for LEU200

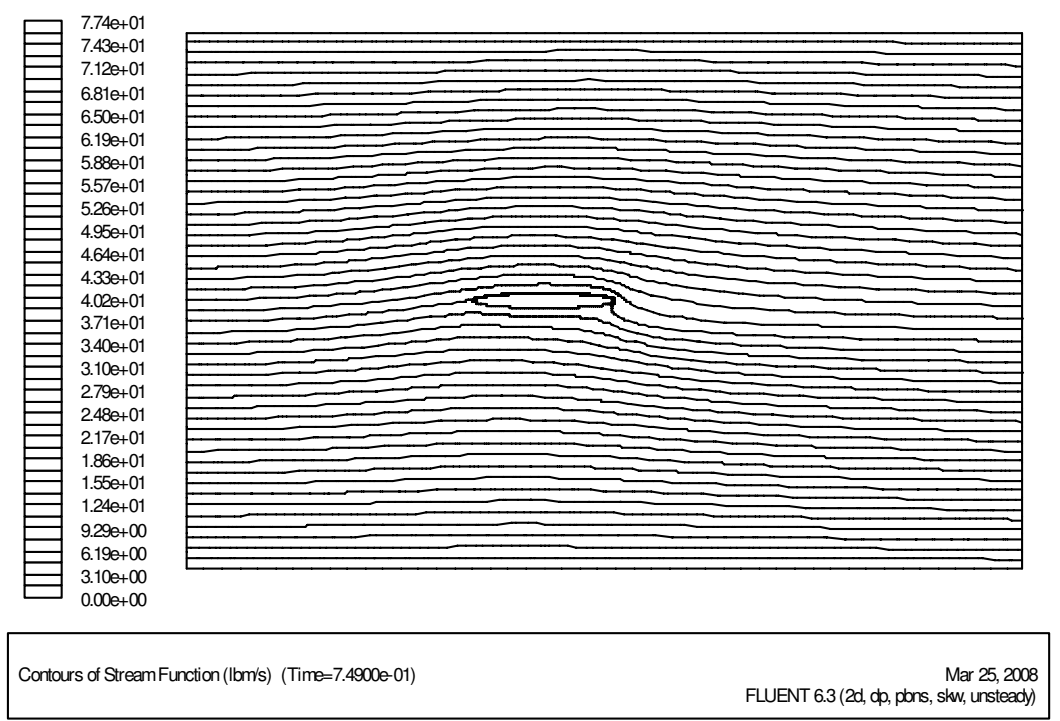

Figure I. 15: Streamlines for LEU200TEU600 


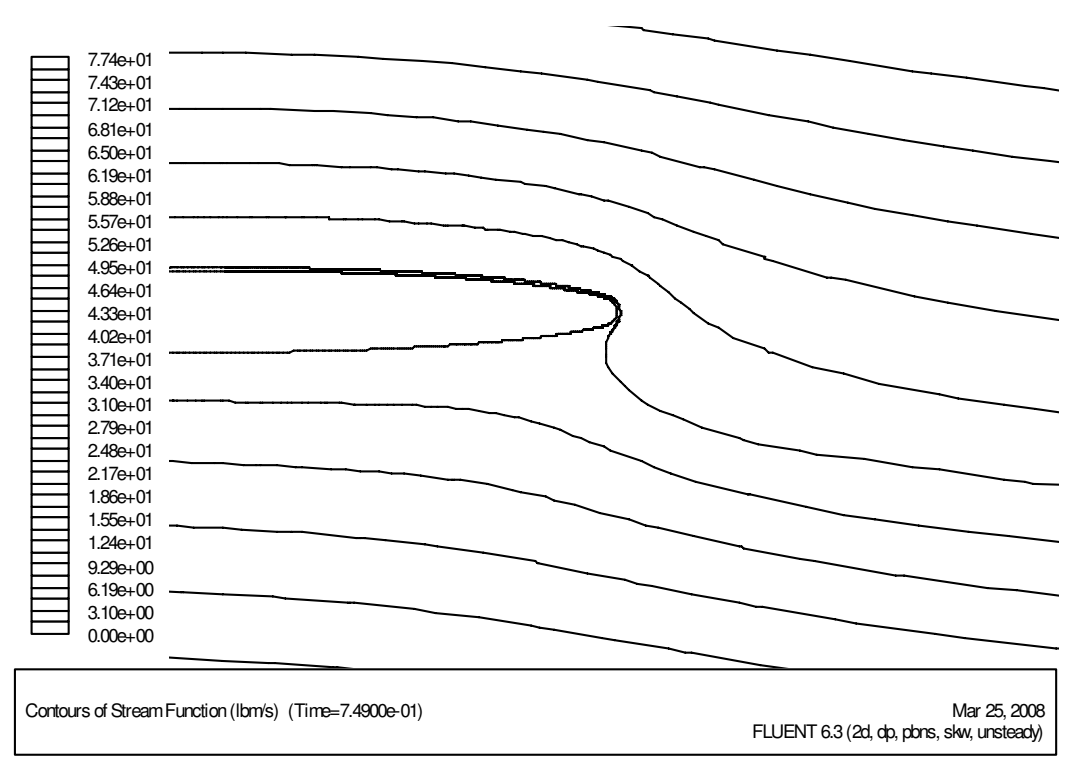

Figure I. 16: Trailing edge streamlines for LEU200TEU600

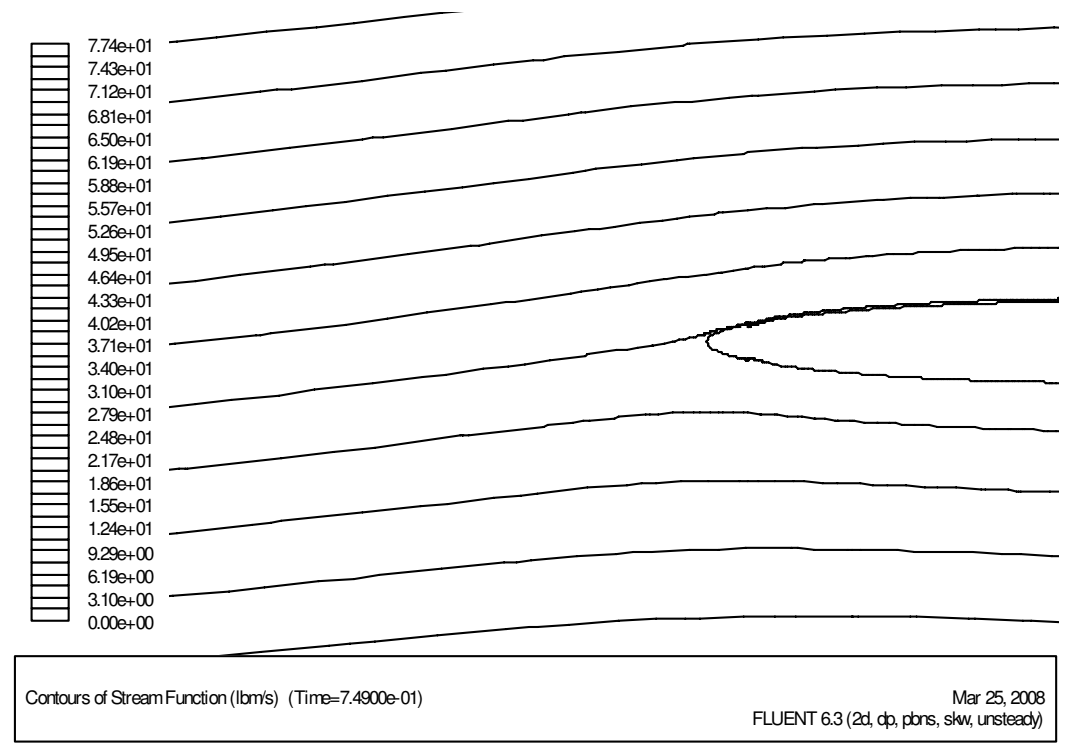

Figure I. 17: Leading edge streamlines for LEU200TEU600 


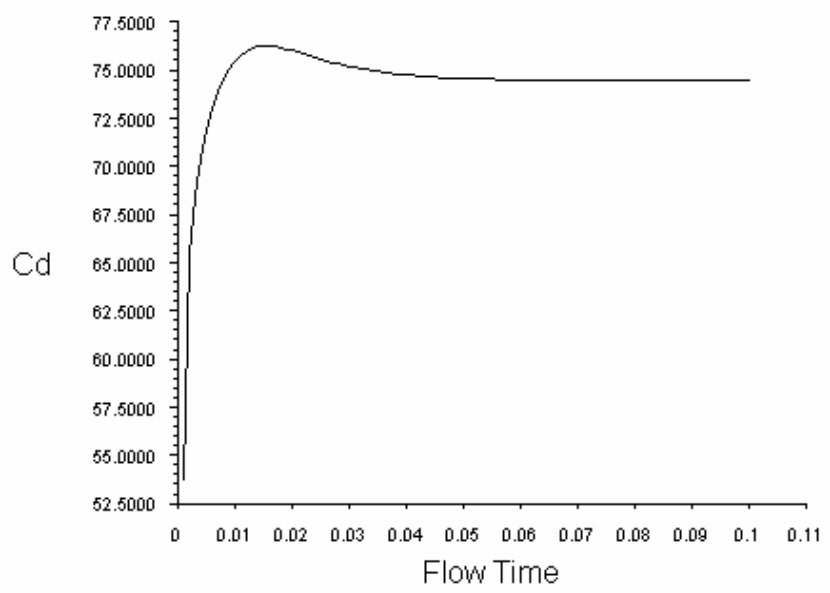

Drag Convergence History (Time $=1.0000 \mathrm{e}-01$ )

Mar 25,2008

FLUENT 6.3 (2d, dp, pbns, skw, unsteady)

Figure I. 18: Drag response characteristics for the lower trailing edge at $400 \mathrm{ft} / \mathrm{s}$ and the upper trailing edge at $800 \mathrm{ft} / \mathrm{s}$

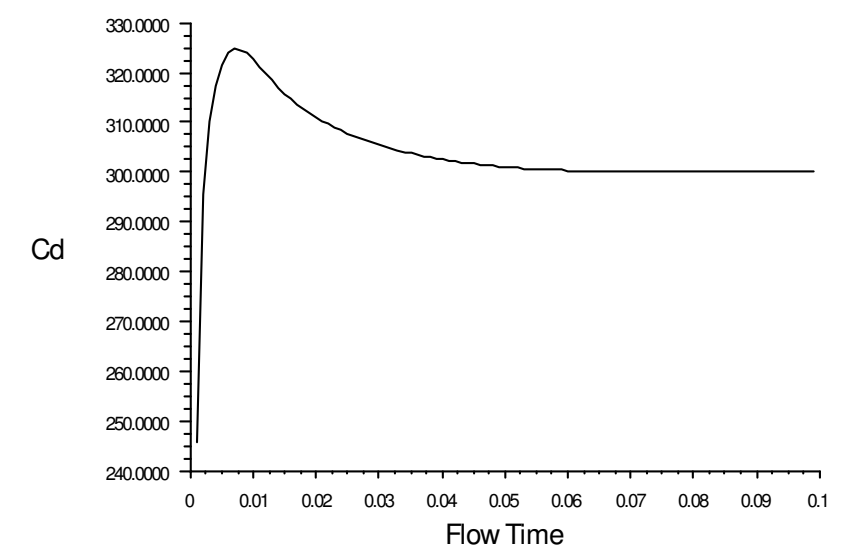

Figure I. 19: Drag response for LEL600TEU1400 


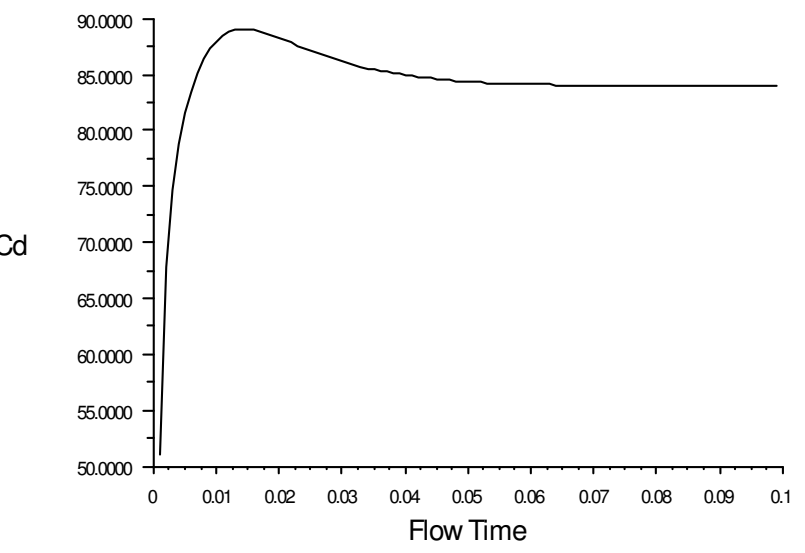

Drag Convergence History (Time=9.9000e-02 Mar 25, 2008
FLUENT 6.3 (2d, dp, pbns, skw, unsteady)

Figure I. 20: Drag response for TEU600

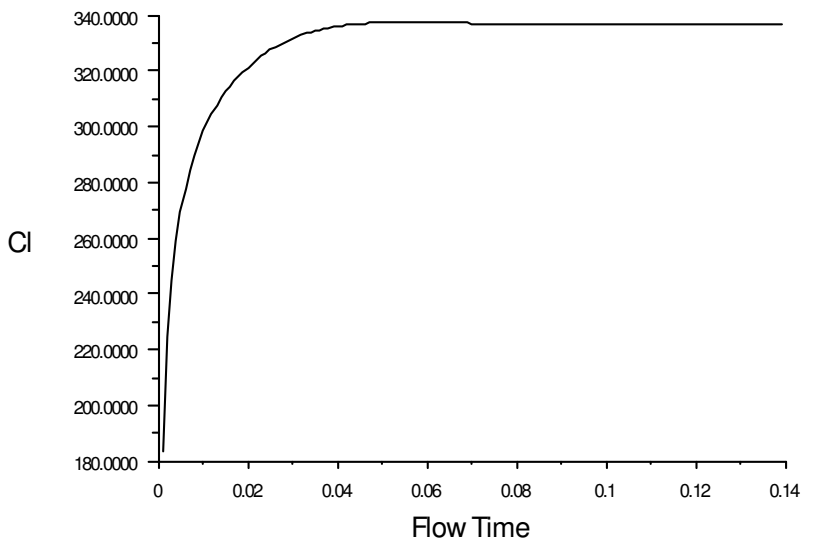

Lift Convergence History (Time=1.3900e-01) FLUENT 6.3 (2d, dp, pbns, skw, unsteady)

Figure I. 21: Lift response for TEU600 


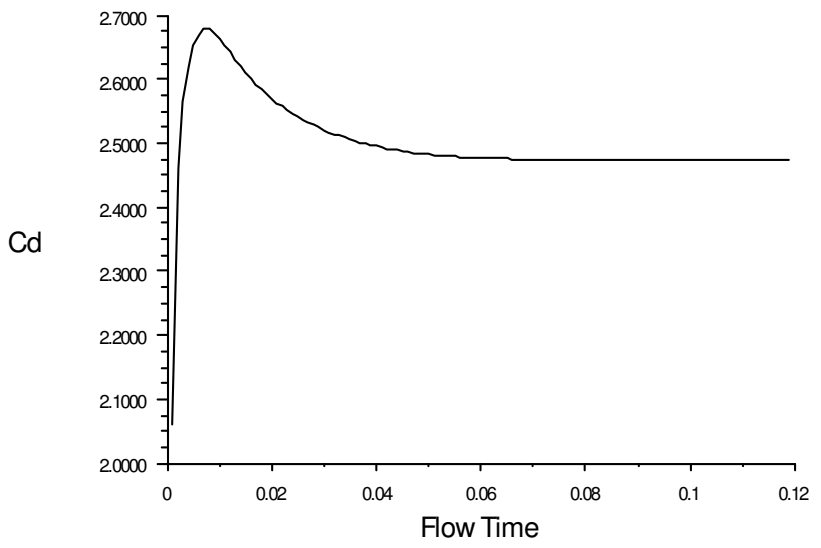

Drag Convergence History (Time=1.1900e-01)

Mar 25, 2008
FLUENT 6.3 (2d, dp, pbns, skw, unsteady)

Figure I. 22: Drag response for LEU200TEU600

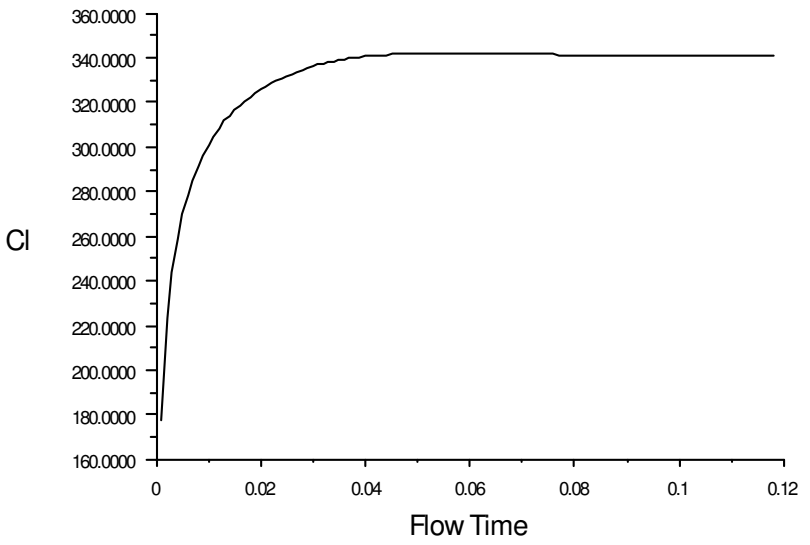

Lift Convergence History (Time=1.1800e-01) FLUENT 6.3 (2d, dp, pbns, skw, unsteady)

Figure I. 23: Lift response for LEU200TEU600 


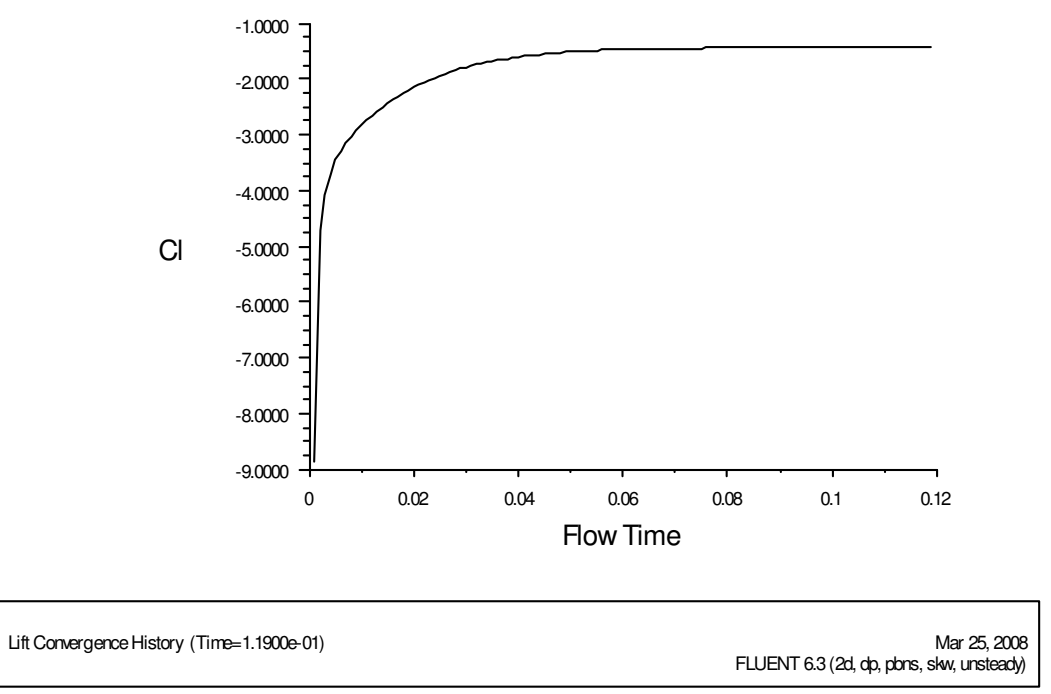

Figure I. 24: Lift response for LEU200

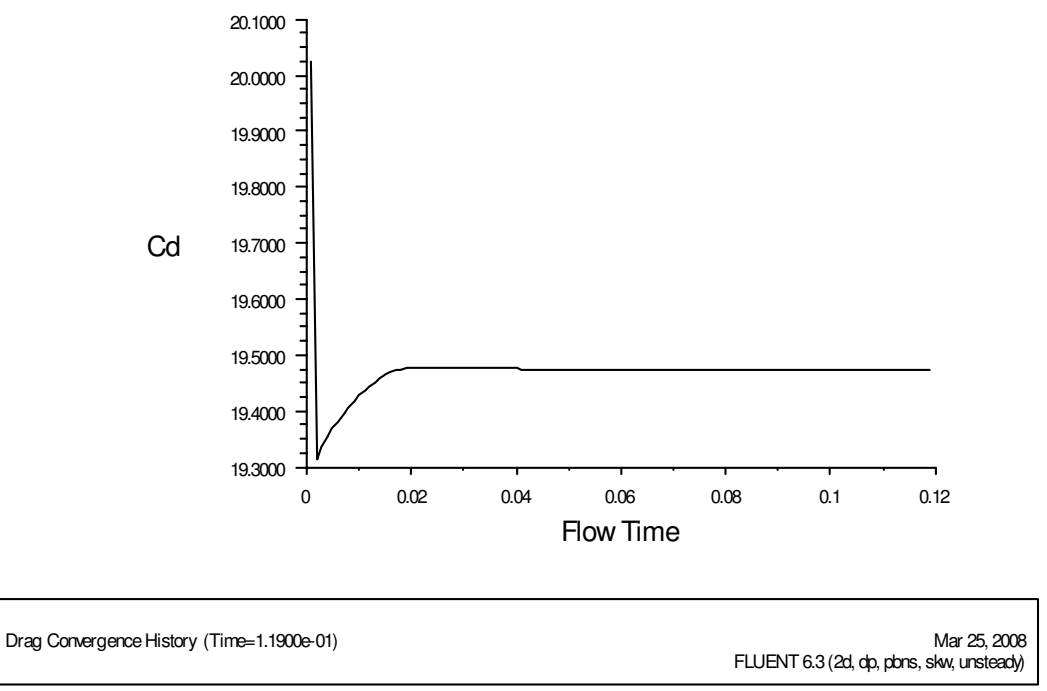

Figure I. 25: Drag response for LEU200 


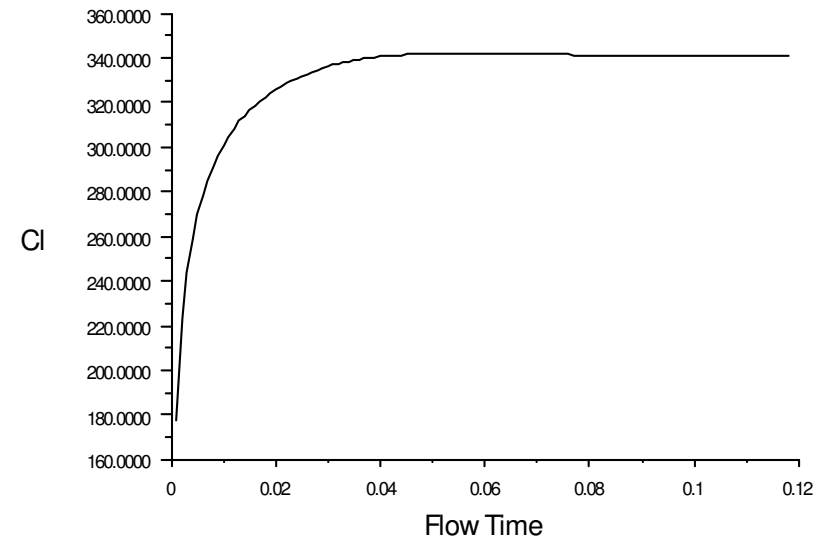

Lift Convergence History (Time=1.1800e-01)

Mar 25, 2008 FLUENT 6.3 (2d, dp, pbns, skw, unsteady)

Figure I. 26: Lift response for LEU200TEU600

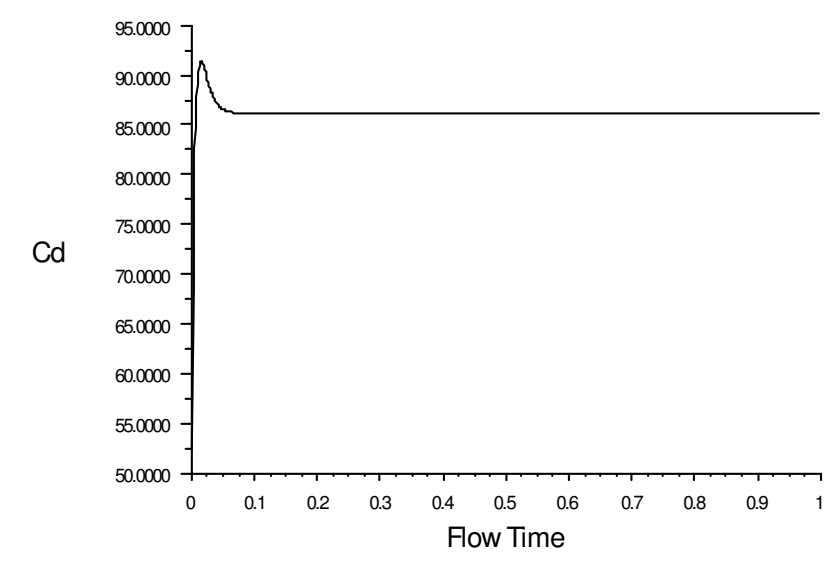

Drag Convergence History (Time=9.9900e 01 ) Mar 25, 2008
FLUENT 6.3 (2d, dp, pbns, skw, unsteady)

Figure I. 27: Drag response for LEU200TEU600 